\title{
Carbon Catabolism in Bacillus subtilis: \\ Global and Molecular Views on the Control of Gene Expression
}

\author{
Dissertation zur Erlangung des Doktorgrades \\ der Mathematisch-Naturwissenschaftlichen Fakultäten \\ der Georg-August-Universität zu Göttingen
}

vorgelegt von

Oliver Schilling

aus Bamberg

Göttingen 2007 
D 7

Referent: Prof. Dr. Jörg Stülke

Korreferent: Prof. Dr. Ralf Ficner

Tag der mündlichen Prüfung: 05.07.07 


\section{Danksagung}

An erster Stelle möchte ich ganz herzlich Prof. Dr. Jörg Stülke für die Stellung des interessanten Themas und für die gute Betreuung der Arbeit danken. Er hat mir für die Verwirklichung meiner Ideen alle nötigen Freiheiten gelassen und brachte mir dadurch großes Vertrauen entgegen. Gleichwohl konnte ich immer auf seine Hilfsbereitschaft zählen, wenn sie gebraucht wurde.

Desweiteren bedanke ich mich bei Herrn Prof. Dr. Ralf Ficner für die freundliche Übernahme des Korreferats und für die Zusammenarbeit bei einem Projekt zur strukturellen Aufklärung des GlcT-RAT Komplexes. Für die finanzielle Unterstützung der Arbeit bedanke ich mich beim Fonds der Chemischen Industrie und bei der Deutschen Forschungsgemeinschaft.

Bei Dr. Armin Ehrenreich bedanke ich mich für die Einführung in die Welt der Microarrays und für seine stete Hilfe bei allen technischen Fragen rund ums Spotten, Labeln und Hybridisieren. Prof. Dr. Elmar Heinzle, PD Dr. Christoph Wittmann und Oliver Frick danke ich für die wertvolle Zusammenarbeit, aber auch dafür, dass ich eine Woche in ihrer Arbeitsgruppe an der Universität Saarbrücken mitarbeiten durfte. In diesem Zusammenhang bedanke ich mich auch bei Michael Fritz und Tae Hoon Yang für die freundliche Mithilfe bei diesem Projekt. Für die Bereitstellung von Bakterienstämmen bedanke ich mich bei Prof. Dr. Richard Herrmann (Universität Heidelberg), Dr. Ulf Gerth (Universität Greifswald) und Dominique Le Coq (Laboratoire de Génétique des Microorganismes, Thiverval-Grignon). Bei Dr. Matthias Görlach und Dr. Matthias Stoldt (Fritz-Lipmann-Institut, Jena) bedanke ich mich für die hilfreichen Gespräche zur NMR-Strukturanalyse. Für ihren experimentellen Beitrag durch Zuckertransport-Messungen möchte ich mich bei PD Dr. Fritz Titgemeyer und Tina Hertrich bedanken.

Ein ganz besonderes Dankeschön geht an Christina Herzberg, die sich in besonderer Weise engagierte und damit wesentlich zu den Ergebnissen in dieser Arbeit beigetragen hat. Sie gehört sicher in die Top-Ten der TAs dieser Welt. Vielen Dank auch für die mentale Unterstützung und all die praktischen Tipps und Ratschläge fürs Leben.

Mein Dank geht auch an Dr. Thorsten Mascher und seine Arbeitsgruppe, welche man immer wieder für ein Spielchen am Kicker begeistern kann. Es war interessant zu beobachten, welch große Fortschritte man dabei in kurzer Zeit erzielen kann. Desweiteren möchte ich mich bei Thorsten für die Einführung in die Tiefen der LFH-PCR bedanken. Bei Dr. Boris Görke bedanke ich mich für die interessanten Diskussionen $\mathrm{zu}$ allerlei molekularbiologischen 
Methoden. Seine Art der praktischen Durchführung ist dabei äußerst konträr zu der, die ich aus dem ursprünglichen „Botchers Lab“ kennengelernt habe.

Prof. Dr. Wolfgang Hillen, an dessen Lehrstuhl an der Universität Erlangen einige Experimente für diese Arbeit durchgeführt wurden, danke ich für die Bereitstellung der dortigen Laborausstattung und Arbeitsumgebung. Den „Erlangern“ Steffi Bachem, HansMatti Blencke, Christian Detsch, Ines Langbein, Michael Müller, Irene Reif, Matthias Schmalisch, Shane Hanson und Ingrid Wacker danke ich für all die kleinen und großen Beiträge zu dieser Arbeit.

Vielen Dank auch an den Erlanger Kern, der mit mir gemeinsam die Reise ins norddeutsche Ausland gewagt hat: Dank an Hans-Matti, der mir in den ersten Wochen in Göttingen ein Dach über dem Kopf gegeben hat, und von dem ich auch gerne etwas von seiner nordischen Gelassenheit übernommen hätte. Dank auch an Fabian Commichau, der mir als erster gezeigt hat, dass es auch außerhalb von Bayern intelligentes Leben gibt. Last but not least danke ich Matthias Schmalisch für seine ausgedehnte Diskussionsbereitschaft zu nahezu jedem Thema unter der Sonne und für seine Überredungskünste, ohne die ich einige Dinge sicher noch nicht ausprobiert hätte (Rollersoccer???). Auch an unseren USA-Aufenthalt werde ich noch lange zurückdenken.

Ein grosses Dankeschön geht aber auch an die "alteingesessenen Göttinger" Birgit Veith und Mark Hoffmeister, die mir zu Beginn in ihrem Labor Asyl gewährt und mich schrittweise in die neuen Gegebenheiten eingeführt haben. Bedanken möchte ich mich natürlich auch bei meinen Praktikanten Timo Hupfeld, Dirk Jessen, Desiree Krausse und Hanna Vörsmann, die ich betreuen durfte, und die teilweise erheblichen Beitrag zu dieser Arbeit geleistet haben. Ich möchte auch meinem ersten und einzigen Diplomanden Sebastian Hübner dafür danken, dass er durch seine selbständige Arbeitsweise wenig Betreuungszeit in Anspruch genommen hat, wovon eigene Projekte profitieren konnten.

Mein Dank gilt selbstverständlich in gleicher Weise auch allen nicht namentlich genannten Kollegen für das gute und lockere Laborklima.

$\mathrm{Zu}$ guter Letzt möchte ich mich noch bei meiner Familie für ihre beständige Unterstützung bedanken. Vielen Dank auch an Kathrin, die mich aufopferungsvoll auch durch schwere Zeiten begleitet und mir die nötige mentale Kraft verliehen hat. 


\section{Table of contents}

List of abbreviations

List of publications

Summary

Zusammenfassung 3

1 Introduction 5

1.1 Sugar metabolism in Bacillus subtilis 5

1.1.1 An overview 5

1.1.2 Regulation of sugar metabolism in B. subtilis: cause and effect 9

1.2 RNA and its role in gene regulation 13

1.2.1 The structural features of single stranded RNA 14

1.2.2 Non-coding RNAs 16

1.2.3 RNA switches 18

1.2.3.1 Regulation by RNA thermometers 21

1.2.3.2 RNA mediated regulation 22

1.2.3.3 Metabolite mediated regulation 23

1.2.3.4 Protein mediated regulation 26

1.2.3.5 RNA aptamers: control by artificial regulatory RNA elements 27

1.3 PTS-controlled antitermination in B. subtilis 29

1.3.1 The PTS and its role in sugar transport 29

1.3.2 Regulation of the $p t s G H I$ operon by transcriptional antitermination 30

1.3.3 The family of homologous antiterminator systems of $B$. subtilis 33

1.4 Aim of this work 36

2 Transcriptional and metabolic responses of Bacillus subtilis to the availability of organic acids: Transcription regulation is important but not sufficient to account for metabolic adaptation

3 Multiple-mutation reaction: A method for the simultaneous introduction of multiple mutations into the glpK gene of Mycoplasma pneumoniae

4 A protein-dependent riboswitch controlling ptsGHI operon expression in Bacillus subtilis: RNA structure rather than sequence provides interaction specificity

5 Keeping signals straight in transcription regulation: specificity determinants for the interaction of a family of conserved bacterial RNA-protein couples 
6 Discussion 121

6.1 Effects of organic acids on the central metabolism of $B$. subtilis 121

6.2 Specificity in gene regulatory systems 131

6.3 Outlook 142

7 References 143

8 Supplementary material 176

Curriculum vitae 205 


\section{List of abbreviations}

\begin{tabular}{|c|c|}
\hline A & adenine \\
\hline asRNA & antisense RNA \\
\hline ATP & adenosine triphosphate \\
\hline BSA & bovine serum albumin \\
\hline bp & base pair \\
\hline $\mathrm{C}$ & carbon \\
\hline $\mathrm{C}$ & cytosine \\
\hline cDNA & copy DNA \\
\hline CCR & carbon catabolite repression \\
\hline CCR & combined chain reaction \\
\hline $\mathrm{cm}$ & centimeter \\
\hline $\mathrm{Cm}$ & chloramphenicol \\
\hline CTT & cytidine triphosphate \\
\hline $\mathrm{Cy} 3 / \mathrm{Cy} 5$ & fluorescent dyes of the cyanine dye family \\
\hline DHAP & dihydroxyacetone phosphate \\
\hline DIG & digoxigenin \\
\hline DNA & desoxyribonucleic acid \\
\hline DNase & desoxyribonuclease I \\
\hline dsRNA & double stranded RNA \\
\hline EI & enzyme I \\
\hline EII & enzyme II \\
\hline EDTA & ethylenediaminetetraacetic acid \\
\hline Em & erythromycin \\
\hline FAD & flavin adenine dinucleotide \\
\hline FBP & fructose-1,6-bisphosphate \\
\hline FMN & flavin mononucleotide \\
\hline Fig. & figure \\
\hline G & guanine \\
\hline Glc & glucose \\
\hline GlcN6P & glucosamine-6-phosphate \\
\hline GMP & guanosine monophosphate \\
\hline GTP & guanosine triphosphate \\
\hline $\mathrm{H}$ & hydrogen \\
\hline $\mathrm{h}$ & hour \\
\hline IMP & inosine monophosphate \\
\hline IPTG & isopropyl- $\beta$-D-1-thiogalactopyranoside \\
\hline $\mathrm{kb}$ & kilo base \\
\hline $\mathrm{kDa}$ & kilodalton \\
\hline $\mathrm{Km}$ & kanamycin \\
\hline LB & Luria Bertani \\
\hline LFH & long flanking homology \\
\hline Lin & lincomycin \\
\hline$\mu 1$ & microliters \\
\hline$\mu \mathrm{m}$ & micrometers \\
\hline M & molar \\
\hline $\mathrm{mCi}$ & milli Curie \\
\hline MCS & multiple cloning site \\
\hline $\mathrm{mg}$ & milligram \\
\hline
\end{tabular}




\begin{tabular}{|c|c|}
\hline $\min$ & minutes \\
\hline miRNA & micro RNA \\
\hline $\mathrm{mj}$ & milli Joule \\
\hline $\mathrm{ml}$ & milliliter \\
\hline $\mathrm{mM}$ & millimolar \\
\hline MMR & multiple mutation reaction \\
\hline mRNA & messenger RNA \\
\hline $\mathrm{mU}$ & milliunit \\
\hline $\mathrm{m} / \mathrm{z}$ & ratio of mass to charge \\
\hline $\mathrm{NAD}^{+} / \mathrm{NADH}$ & $\beta$-nicotinamide adenine dinucleotide \\
\hline NADPH & $\beta$-nicotinamide adenine dinucleotide phosphate \\
\hline ncRNA & non-coding RNA \\
\hline ng & nanogram \\
\hline $\mathrm{nm}$ & nanometer \\
\hline NTA & nitrilotriacetic acid \\
\hline OD & optical density \\
\hline PAA & polyacrylamide \\
\hline PAGE & polyacrylamide gel electrophoresis \\
\hline PCR & polymerase chain reaction \\
\hline PEP & phosphoenolpyruvate \\
\hline pg & picograms \\
\hline $\mathrm{pH}$ & power of hydrogen \\
\hline pl & picoliter \\
\hline pM & pico molar \\
\hline pmol & picomole \\
\hline PPP & pentose phosphate pathway \\
\hline PRD-I & PTS regulation domain I \\
\hline PRD-II & PTS regulation domain II \\
\hline PTS & phosphoenolpyruvate:sugar phosphotransferase system \\
\hline pyr & pyrimidine \\
\hline PVDF & polyvinylidene difluoride membrane \\
\hline RAT & RNA antiterminator \\
\hline RBD & RNA-binding domain \\
\hline RNA & ribonucleic acid \\
\hline RNAi & RNA interference \\
\hline ROSE & repression of heat-shock gene expression \\
\hline rRNA & ribosomal RNA \\
\hline S & second \\
\hline Sal & salicin \\
\hline SAM & S-adenosylmethionine \\
\hline SD & Shine Dalgarno \\
\hline SDS & sodium dodecyl sulfate \\
\hline SELEX & systematic evolution of ligands by exponential enrichment \\
\hline siRNA & small interfering RNA \\
\hline $\mathrm{Spc}$ & spectinomycin \\
\hline sRNA & small RNA \\
\hline Suc & sucrose \\
\hline $\mathrm{T}$ & thymine \\
\hline TAE & tris acetate EDTA \\
\hline TCA & tricarboxylic acid \\
\hline $\mathrm{TE}$ & Tris-EDTA \\
\hline
\end{tabular}




$\begin{array}{ll}\text { Tm } & \text { melting temperature } \\ \text { TPP } & \text { thiamine pyrophosphate } \\ \text { TRAP } & \text { trp RNA-binding attenuation protein } \\ \text { Tris } & \text { tris(hydroxymethyl)aminomethane } \\ \text { tRNA } & \text { transfer RNA } \\ \text { trp } & \text { tryptophan } \\ \text { TTP } & \text { thymidine triphosphate } \\ \text { U } & \text { uracile } \\ \text { U } & \text { unit } \\ \text { UMP } & \text { uridyl monophosphate } \\ \text { UTP } & \text { uridyl triphosphate } \\ \text { UTR } & \text { untranslated region } \\ \text { UV } & \text { ultraviolet } \\ \text { X-Gal } & \text { 5-Bromo-4-chloro-3-indolyl- } \beta \text {-D-galactopyranoside } \\ \text { WT } & \text { wild type } \\ \text { wt/vol } & \text { weight per volume }\end{array}$




\section{List of publications}

Schilling, O., I. Langbein, M. Müller, M. H. Schmalisch and J. Stülke. 2004. A proteindependent riboswitch controlling ptsGHI operon expression in Bacillus subtilis: RNA structure rather than sequence provides interaction specificity. Nucleic Acids Res 32:28532864.

Hames, C., S. Halbedel, O. Schilling and J. Stülke. 2005. Multiple-mutation reaction: a method for simultaneous introduction of multiple mutations into the $g l p K$ gene of Mycoplasma pneumoniae. Appl Environ Microbiol 71:4097-4100.

Schilling, O., C. Herzberg, T. Hertrich, H. Vorsmann, D. Jessen, S. Hübner, F. Titgemeyer and J. Stülke. 2006. Keeping signals straight in transcription regulation: specificity determinants for the interaction of a family of conserved bacterial RNA-protein couples. Nucleic Acids Res 34:6102-6115.

Schilling, O., O. Frick, C. Herzberg, A. Ehrenreich, E. Heinzle, C. Wittmann and J. Stülke. 2007. Transcriptional and metabolic responses of Bacillus subtilis to the availability of organic acids: Transcription regulation is important but not sufficient to account for metabolic adaptation. Appl Environ Microbiol 73:499-507.

Vogl, C., S. Grill, O. Schilling, J. Stülke, M. Mack and J. Stolz. 2007. Characterization of riboflavin (vitamin $\mathrm{B}_{2}$ ) transport proteins from Bacillus subtilis and Corynebacterium glutamicum. Submitted to J. Bacteriol. 


\section{Summary}

The soil bacterium Bacillus subtilis is exposed to frequently changing environmental conditions. Moreover, in its natural habitat, B. subtilis is surrounded by numerous microorganisms that are competing for the available resources. Therefore, the survival strategy of $B$. subtilis is optimized towards a rapid utilization of the available nutrients. The uptake of glucose, which is the preferred carbon source of B. subtilis, is mediated by the phosphoenolpyruvate:phosphotransferase system (PTS) encoded by the ptsGHI operon. The expression of this operon is controlled by transcriptional antitermination. The mRNA of the ptsGHI operon can adopt one of two alternative secondary structures in its 5'-untranslated region. The formation of a thermodynamic more stable terminator causes premature transcription termination. However, when the antiterminator protein GlcT binds to this region, it stabilizes an antiterminator structure called RAT and thus prevents terminator formation. The activity of GlcT is controlled by the availability of glucose. Only in the presence of glucose, GlcT can bind and stabilize the RAT structure. There are three more PTS-dependent antitermination systems in B. subtilis. These systems are controlled by the availability of sucrose or $\beta$-glucosides. Both the antiterminator proteins as well as the respective RATs are all very similar in structure and sequence. Nevertheless, the regulation by the antiterminator proteins is highly specific.

The aim of this work was the detection of all specificity determinants of the related antitermination systems. Site directed mutagenesis of two different RATs should help to identify nucleotides that are essential for the specific interaction with the respective antiterminator proteins. Furthermore, it was tested if the specificity of certain RATs can be changed towards other antiterminator proteins. For the simultaneous introduction of multiple point mutations, a method called MMR (multiple mutation reaction) was established. All nucleotides essential for specificity are located in the lower loop regions of the related RAT structures. Site directed mutagenesis of this region could also change specificity of certain RATs towards other antiterminator proteins. The interaction of the antiterminator proteins with their respective permeases is also specific and contributes to the overall specificity of the systems. Furthermore, it was found that carbon catabolite repression is also essential for the maintenance of specificity and for preventing cross-talk among the different systems.

For the utilization of its preferred carbon source glucose, B. subtilis features a complete set of enzymes for glycolysis, the pentose phosphate shunt, the tricarboxylic acid cycle and the respiratory chain. When available, glucose causes the repression of genes 
needed for the utilization of alternative carbon sources. Only when glucose is completely exhausted, the genes necessary for the utilization of these carbon sources are expressed. This effect is known as carbon catabolite repression and has already been intensively studied.

The second aim of this work was to determine the effect of the organic acids glutamate and succinate on the central metabolism of B. subtilis when given in addition to glucose. This was achieved by a combined approach using transcriptomic data and metabolic flux analysis. The results of both studies were in good agreement for most of the studied genes. The addition of glutamate and succinate had no major effect on the genes of glycolysis and the pentose phosphate pathway. However, the flux of acetyl-CoA into the tricarboxylic acid cycle was severely reduced. On the other hand, the overflow metabolic pathways of lactate and acetate synthesis were significantly induced. For some of the genes, the change in transcription had not the expected consequence on the metabolic fluxes. This is in particular true for the genes involved in acetoin biosynthesis. Although the relevant genes were highly induced, no formation of acetoin was observed. These differences in the two data sets could indicate that the relevant enzymes are regulated on the level of protein activity. 


\section{Zusammenfassung}

Als Bodenbakterium ist Bacillus subtilis in seinem natürlichen Lebensraum häufig wechselnden Umweltbedingungen ausgesetzt. Zudem ist B. subtilis in seinem Habitat von zahlreichen weiteren Mikroorganismen umgeben, welche mit ihm um die verfügbaren Ressourcen konkurrieren. Zur Überlebensstrategie von B. subtilis gehört es daher, die vorhandenen Nährstoffe möglichst schnell aufzunehmen und $\mathrm{zu}$ verwerten. Hierbei ist Glukose seine bevorzugte Kohlenstoffquelle. Die Aufnahme der Glukose erfolgt über das Phosphoenolpyruvat:Zucker Phosphotransferase System (PTS), welches durch das ptsGHIOperon kodiert wird. Die Expression dieses Operons sowie die Expression einiger weiterer zuckerspezifischer Permeasen werden durch transkriptionelle Antitermination kontrolliert. Hierbei kann die mRNA dieser Gene im 5'-Bereich eine von zwei alternativen Sekundärstrukturen annehmen. Bei Ausbildung des thermodynamisch stabileren Terminators kommt es zum vorzeitigen Abbruch der Transkription. Bindet jedoch das Antiterminatorprotein GlcT an diesen Bereich der mRNA, so wird die AntiterminatorStruktur RAT stabilisiert und die Ausbildung des Terminators verhindert. Die Aktivität von GlcT wird durch die Verfügbarkeit von Glukose reguliert. Nur wenn Glukose vorhanden ist, kann GlcT an das RAT binden und es stabilisieren. In B. subtilis gibt es drei weitere PTS-abhängige Antiterminationssysteme, welche durch das Vorhandensein von Saccharose oder $\beta$-Glucosiden reguliert werden. Sowohl die Antiterminatorproteine als auch die zugehörigen RATs dieser Systeme weisen eine starke Homologie zueinander auf. Dennoch erkennen die Antiterminatorproteine spezifisch ihre jeweilige RAT-Struktur.

In dieser Arbeit sollten die Spezifitätsdeterminanten aller verwandten Antiterminationssysteme gefunden werden. Durch ortsgerichtete Mutagenesen zweier unterschiedlicher RATs sollten die Nukleotide identifiziert werden, welche für die spezifische Interaktion mit dem zugehörigen Antiterminatorprotein essenziell sind. Außerdem sollte getestet werden, ob es möglich ist, die Spezifität zugunsten eines anderen Antiterminatorproteins $\mathrm{zu}$ verändern. Um mehrere ortsgerichtete Mutagenesen parallel durchführen zu können, wurde die MMR (Multiple Mutation Reaction) als neue Methode etabliert. Es konnte gezeigt werden, dass sich die für die Spezifität wichtigen Nukleotide ausschließlich im unteren Schleifen-Bereich der verwandten RAT-Strukturen befinden. Durch gezielte Mutationen in diesem Bereich war es zudem möglich, die Spezifität der RATs zugunsten anderer Antiterminatorproteine $\mathrm{zu}$ verändern. Die Interaktion der Antiterminatorproteine mit ihrer zugehörigen Permease, welche sich für die direkte 
Regulation des jeweiligen Antiterminatorproteins verantwortlich zeigt, ist spezifisch und trägt zur Spezifität des Gesamtsystems bei. Darüber hinaus konnte gezeigt werden, dass auch die Katabolitenrepression essenziell für die Aufrechterhaltung der Spezifität ist und Überschneidungen unter den verwandten Systemen verhindert.

Zur Verwertung seiner bevorzugten C-Quelle Glukose steht B. subtilis der komplette Satz an Enzymen für Glykolyse, Pentose-Phosphat-Weg, Zitronensäure-Zyklus und Atmungskette zur Verfügung. Gleichzeitig reprimiert die Anwesenheit von Glukose die Expression der Gene, welche für die Verwertung von anderen C-Quellen benötigt werden. Erst wenn Glukose verbraucht ist, werden die Gene für die Verwertung anderer C-Quellen induziert. Dieser als Katabolitenrepression bezeichnete Effekt war bereits Mittelpunkt zahlreicher Untersuchungen.

Eine weitere Zielsetzung dieser Arbeit war es daher, bei gleichzeitiger Anwesenheit von Glukose, den Einfluss der organischen Säuren Glutamat und Succinat auf den zentralen Stoffwechsel von B. subtilis zu untersuchen. Hierzu wurden Transkriptomdaten, welche mit DNA-Microarrays gewonnen wurden, sowie Daten aus metabolischen Flussanalysen kombiniert. Die Ergebnisse beider Methoden stimmen für den Großteil der untersuchten Stoffwechselwege sehr gut überein. Die Zugabe von Glutamat und Succinat hat keinen Effekt auf die Gene der Glykolyse und des Pentose-Phosphat-Wegs. Der Zufluss von Acetyl-CoA in den Zitronensäure-Zyklus ist jedoch stark reduziert, wohingegen die Aktivität der Enzyme für die Überfluss-Stoffwechselwege der Lactat- und Acetat-Synthese stark erhöht ist. Für einige wenige Gene wirkt sich die Änderung in der Genexpression jedoch nicht wie erwartet auf den metabolischen Fluss aus. Dies gilt besonders für die Gene der Acetoin-Biosynthese. Trotz einer starken Induktion der beteiligten Gene konnte keine Synthese von Acetoin beobachtet werden. Derartige Abweichungen können Hinweise darauf geben, dass die Aktivität der beteiligten Enzyme auf Proteinebene reguliert wird. 


\section{Introduction}

\subsection{Sugar metabolism in Bacillus subtilis}

\subsubsection{An overview}

All living organisms face the same challenge: they need energy to maintain their vital functions such as motility, transport of molecules, maintenance of proton motive force and many anabolic reactions. The central metabolism does not only provide the supply of energy rich molecules, but also transforms and modifies them, interconnects different chemical pathways, and feeds the supply of all building blocks of the living cell. Nitrogen metabolism, for example, is of great importance for the supply of amino acids and nitrogen containing molecules like purines and pyrimidines for DNA and RNA synthesis. Thus, the central metabolism plays a key role in all processes of life.

Many metabolic pathways, some organism-specific, catalyze the conversion of various metabolites to obtain a continuous synthesis of ATP, NADH and NADPH. These pathways, among them glycolysis, the pentose phosphate pathway, the citric acid cycle, the Calvin cycle, and photosynthesis, can be found in many organisms of prokaryotic and eukaryotic origin.

Among bacteria, the Gram-positive soil bacterium Bacillus subtilis is one of the best studied organisms. In its natural habitat, the upper part of the soil and the rhizosphere, the bacterium has access to a variety of plant remains, containing diverse energy rich molecules. B. subtilis features a complete genomic configuration to utilize carbohydrates as its preferred carbon and energy source. Excreted enzymes, such as amylases and glucanases, help to make long chain sugar molecules like starch and cellulose available for the organism. Sugar uptake is mediated by facilitators, primary and secondary active transporters, and the phosphoenolpyruvate:sugar phosphotransferase system (PTS) (Postma et al., 1993; Saier et al., 2002; Simoni et al., 1967).

Among these, the PTS is the most important sugar uptake system. It consists of the two general proteins enzyme I (EI) and HPr, and several sugar specific enzymes (see chapter 1.1.2 and 1.3). These membrane-bound sugar specific permeases (enzyme II or EII) not only mediate sugar uptake, but also modify the sugar by phosphorylation (Reizer et al., 1988). Phosphorylated sugars are negatively charged and cannot leave the cell anymore. Thus, they are captured inside the cell. The PTS represents an energy efficient way for sugar uptake and 
for making sugars available for downstream metabolic pathways like glycolysis and the pentose phosphate pathway.

Among the sugars, glucose is the preferred carbon source of B. subtilis. Its uptake is mainly mediated by the glucose specific EII ${ }^{\mathrm{Glc}}$ of the PTS. The resulting glucose-6-phosphate can directly feed into glycolysis. B. subtilis features all glycolytic enzymes needed to convert glucose to pyruvate (Ludwig et al., 2001). Glucose, that was not transported via the PTS or that is derived from hydrolysis of other oligo- or disaccharides, e.g. sucrose, has to be phosphorylated by the glucose kinase (Skarlatos and Dahl, 1998). The next step is the conversion of glucose-6-phosphate to fructose-1,6-bisphosphate which is then split into two C-3 molecules, dihydroxyacetone phosphate (DHAP) and glyceraldehyde-3-phosphate (see Fig. 1.1). Accumulated DHAP is also converted to glyceraldehyde-3-P. The enzymes needed for the conversion of glyceraldehyde-3-phosphate to phosphoenolpyruvate are organized in an operon (gapA operon) on the B. subtilis chromosome (Fillinger et al., 2000; Leyva-Vazquez and Setlow, 1994) (see Chapter 1.1.2). The last step is the conversion of PEP to pyruvate, and the net outcome of the glycolysis are 2 molecules of ATP and 2 molecules NADH.

Glucose-6-phosphate is important for the synthesis of many polysaccharides e.g. peptidoglycan which is needed for cell wall synthesis. The glycolytic intermediates 3-phosphoglycerate, PEP, and pyruvate are significant as sources for the synthesis of all aromatic amino acids, and for the amino acids of the alanine and serine families. Many enzymes of glycolysis work also reversibly and are therefore part of the gluconeogenesis. The only glycolytic enzymes that do not work reversibly are $p t s G, g a p A, p f k$, and $p y k A$ (see Fig. $1.1)$.

In the absence of glucose, gluconeogenesis forms new glucose from metabolic intermediates such as oxaloacetic acid or dihydroxyacetone phosphate. Gluconeogenesis starts with the conversion of oxaloacetate to PEP, carried out by the phosphoenolpyruvate carboxykinase $(p c k A)$. This gluconeogenetic reaction is essential when the organism grows on TCA cycle intermediates (Diesterhaft and Freese, 1973; Yoshida et al., 2001). Although the B. subtilis genome codes for a phosphoenolpyruvate synthase (pps), the bacterium, in contrast to Escherichia coli, does not seem to be able to directly convert pyruvate to PEP (Sauer and Eikmanns, 2005). To synthesize glucose-6-P from PEP two further enzymes are necessary, since gap $A$ and $p f k$ do not work reversibly (see Fig. 1.1). 


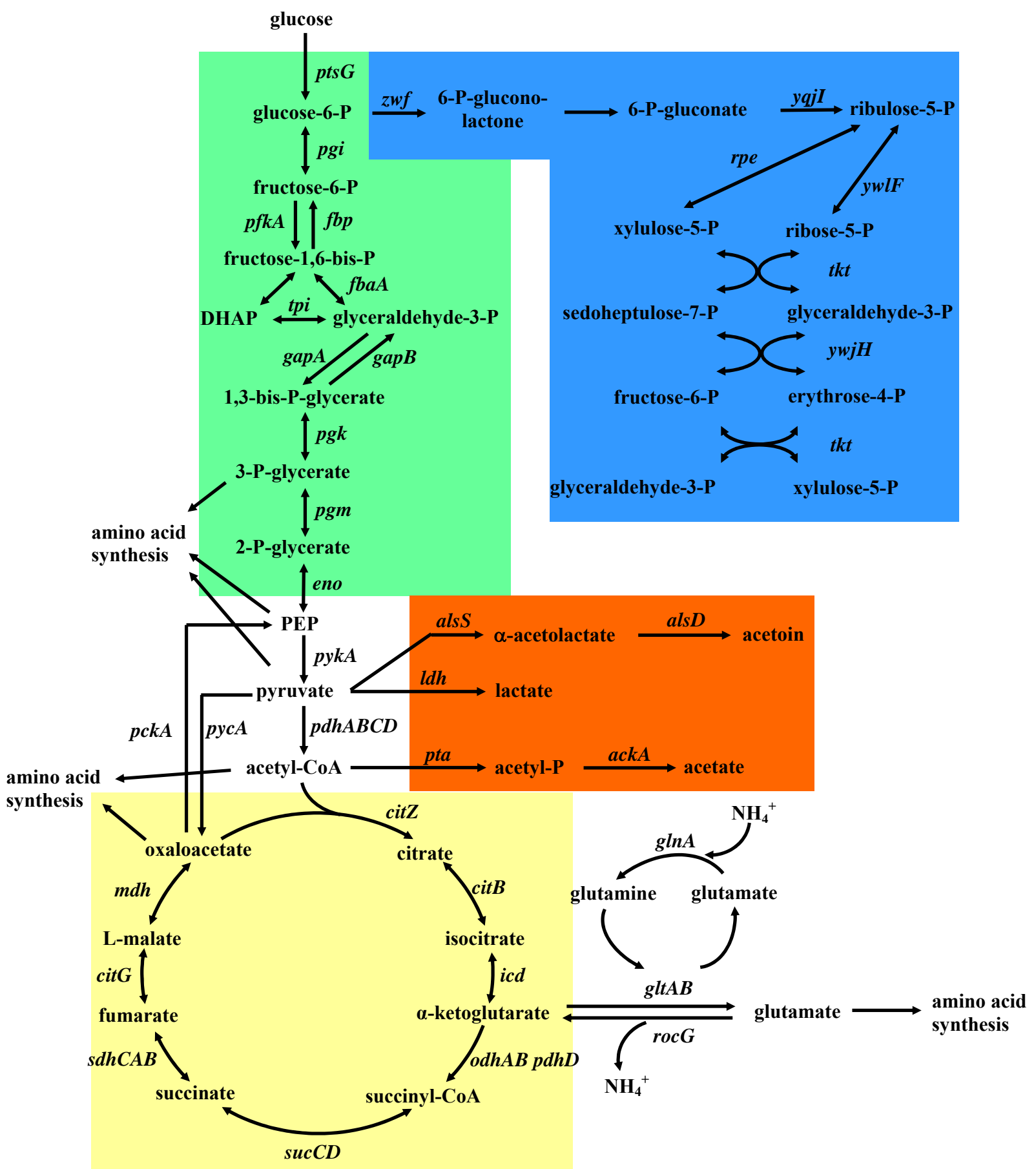

Figure 1.1. Overview on the genes of central metabolic pathways in B. subtilis.

Glycolysis is indicated in green, the pentose phosphate shunt is indicated in blue, overflow metabolic pathways are indicated in red, and the citric acid cycle is indicated in yellow. Modified from Blencke et al., 2003. 
The synthesis of glyceraldehyde-3-P from 1,3-bisphosphoglycerate is catalyzed by the glyceraldehyde-3-phosphate dehydrogenase $(\operatorname{gapB})$, and fructose-6-phosphate is formed from fructose-1,6-bisphosphate by the activity of the fructose-1,6-bisphosphatase $(f b p)$ (Fillinger et al., 2000; Fujita et al., 1998; Servant et al., 2005).

The pentose phosphate pathway (PPP) provides precursors for DNA, RNA, and amino acid syntheses (Tozzi et al., 2006). Furthermore, it has an important role in providing NADPH for anabolic reactions. The pentose phosphate pathway can be subdivided into an oxidative and a non-oxidative part. In the oxidative part, glucose-6-phosphate is converted to ribulose-5-phosphate and 2 molecules of NADPH are synthesized. In the non-oxidative stage, it can interconvert different types of pentoses and additionally converts pentoses to hexoses (fructose-6-phosphate) and trioses (glyceraldehyde-3-phosphate) that can feed into glycolysis (see Fig. 1.1). Depending on what metabolic intermediates are needed, the pentose phosphate pathway can flow into the one or the other direction. In contrast to its important role for B. subtilis, the PPP was not too much in the focus of scientific research. Therefore, it was only recently discovered that the phosphogluconate dehydrogenase is coded by yqjI, and not gntZ, as assumed previously (Zamboni et al., 2004).

Pyruvate is the final product of glycolysis, but it is also generated when PEP is used as a phosphate donor to power the sugar transport of the PTS. Pyruvate can be used to produce lactate via the lactate dehydrogenase $(l d h)$ or acetoin via the acetolactate synthase and acetolactate decarboxylase (alsSD operon) (Renna et al., 1993). These reactions are part of the overflow metabolism, which helps the bacterium to regenerate $\mathrm{NAD}^{+}$from $\mathrm{NADH}$ when no other electron acceptors like oxygen or nitrate are available (Cruz Ramos et al., 2000). B. subtilis uses overflow metabolic pathways also under aerobic conditions, when excess of carbon and nitrogen sources are present (Shivers et al., 2006).

Pyruvate can also be converted to acetyl coenzyme A (acetyl-CoA) by the pyruvate dehydrogenase complex ( $p d h A B C D$ ) (Hemilä et al., 1991). Acetyl-CoA can feed into the TCA cycle, is used for synthesis of the amino acids lysine and methionine, or is converted to acetyl phosphate and further to acetate via phosphotransacetylase (pta) and acetate kinase (ackA, see Fig. 1.1) (Grundy et al., 1993; Presecan-Siedel et al., 1999; Shin et al., 1999). The latter reactions are also part of overflow metabolism.

B. subtilis features the complete enzymatic configuration for a cyclic TCA pathway (Huynen et al., 1999). Under aerobic conditions, the TCA cycle catalyzes the complete oxidation of pyruvate to $\mathrm{CO}_{2}$, and hydride ions are transferred to electron carrier molecules. One molecule of pyruvate leads to the formation of $4 \mathrm{NADH}$ (or $3 \mathrm{NADH}$ and $1 \mathrm{NADPH}$ ), 
and 1 FADH. Moreover, 1 GTP molecule is formed per cycle. The energy bound in these hydrogen atom carrier molecules $\left(\mathrm{NAD}^{+}, \mathrm{FAD}^{+}\right)$is used to generate ATP via the respiratory chain (Hederstedt, 1993; Sonenshein, 2002). The provided NADPH can be used in anabolic reactions.

Another important task of the TCA cycle is the supply of precursors for amino acid biosynthesis such as $\alpha$-ketoglutarate, and oxaloacetate (see Fig. 1.1). $\alpha$-Ketoglutarate is the precursor of glutamate, which itself is the precursor of many other amino acids (Schreier, 1993). The synthesis of glutamate is performed by the transfer of an amino group to $\alpha$-ketoglutarate by the glutamate synthase (glt $A B$ operon). This reaction represents the link between C- and N-metabolism in B. subtilis (Commichau et al., 2006). Oxaloacetate is used as a precursor for the synthesis of the amino acids of the aspartate family (Akashi and Gojobori, 2002). To ensure a continuous function of the TCA cycle, the flow of TCA intermediates has to be substituted. This can be achieved either by the reversion of the above-mentioned reactions, or by directly carboxylating pyruvate to oxaloacetate, a reaction that requires energy in form of ATP. This so called anaplerotic reaction is carried out by the pyruvate carboxylase (pycA) in B. subtilis (Bernhardt et al., 2003; Sonenshein, 2002). A third way to refill TCA cycle intermediates is the generation of succinyl-CoA by the $\beta$-oxidation of odd-chain fatty acids. Due to the lack of a glyoxylate cycle, B. subtilis is not able to grow on acetate as its only carbon source (Huynen et al., 1999).

\subsubsection{Regulation of sugar metabolism in B. subtilis: cause and effect}

B. subtilis can utilize a huge variety of different sugars as carbon and energy source. The transcription of the respective gene needed to metabolize a certain sugar is only initiated if the particular carbohydrate is available (Stülke and Hillen, 2000). However, not all carbon sources deliver the same amount of energy. Therefore, if a multitude of sugars is available, only the optimal carbon source is metabolized. This mode of gene regulation is described as carbon catabolite repression (CCR) (Chambliss, 1993).

The regulation of sugar metabolism in B. subtilis already starts at the uptake process, and here the PTS plays a crucial role (see chapter 1.3). Besides its important role in sugar uptake, the PTS also has a major impact on the regulation of downstream metabolic processes. Among the general PTS components, the HPr protein plays the key role. It can be phosphorylated at positions His-15 and Ser-46 (Galinier et al., 1998; Stülke and Hillen, 2000). HPr (His-P) serves as a phosphate donor for the phosphorylation of proteins involved in the regulation of sugar permeases and inducer exclusion (Stülke and Schmalisch, 2004). In 
contrast to HPr (His-P), HPr (Ser-P) does not serve as a phosphate donor, but acts as a co-repressor of the global transcription regulatory protein CcpA (see below). The Ser-46 phosphorylation is mediated by the enzyme HPr kinase/phosphorylase (hprK) (Deutscher et al., 1986; Deutscher and Saier, 1983). HPrK/P senses the concentration of intracellular ATP and glycolytic intermediates such as fructose-1,6-bisphosphate (FBP). FBP stimulates the kinase activity of HPrK/P, whereas inorganic phosphate stimulates the phosphorylase activity (Hanson et al., 2002; Jault et al., 2000; Ramström et al., 2003). Thus, HPrK/P controls the HPr phosphorylation state subject to the energy charge of the cell (Galinier et al., 1998; Ludwig et al., 2002b; Reizer et al., 1998). There is also indication that the activity of HPrK/P is influenced by the intracellular $\mathrm{pH}$ value of the cell (Ramström et al., 2003).

CcpA (carbon catabolite control protein A) is the main regulatory protein of carbon metabolism in B. subtilis and other Gram-positive bacteria. CcpA belongs to the LacI/GalR family of transcription regulatory proteins (Henkin, 1996; Weickert and Adhya, 1992). It consists of an N-terminal DNA binding domain and two C-terminal subdomains. The CcpA protein is a dimer of two identical subunits (Jones et al., 1997; Schumacher et al., 1994). It is assumed that over 300 genes are subjected to CcpA dependent regulation, which is almost $10 \%$ of the total number of B. subtilis genes (Blencke et al., 2003; Moreno et al., 2001; Yoshida et al., 2001).

There are two modes of CcpA dependent regulation. CcpA can influence the transcription of catabolic genes and operons when it binds to a palindromic sequence called catabolite responsive element (cre sequence). This was first discovered in studies on the regulation of the $\alpha$-amylase gene in $B$. subtilis and a consensus sequence for cre was determined (Henkin et al., 1991; Hueck et al., 1994; Kim et al., 1995; Miwa et al., 2000). The intrinsic interaction of cre and CcpA is stimulated when HPr (Ser-P) binds to CcpA. The HPr analog Crh can also bind to CcpA. Hence, HPr and Crh act both as cofactors in CcpA dependent regulation. Unlike HPr, Crh can not be phosphorylated at position His-15 and is therefore not part of the PTS (Galinier et al., 1999; Galinier et al., 1997). Regulation that is mediated by the binding of CcpA to a cre sequence is defined as class I regulation. The complex of CcpA and HPr/Crh (Ser-P) can act both as a repressor and as an activator of gene expression (see below). In a CcpA mutant strain, differential gene expression occurs also for genes that do not have a cre sequence. Since a functional CcpA protein is necessary for efficient sugar transport, the absence of CcpA affects the function of the PTS and hence the glucose uptake rate. Genes controlled by regulators that sense glucose or its metabolic intermediates are therefore indirectly influenced by CcpA activity. This indirect mode of 
CcpA dependent regulation caused by limited sugar uptake is defined as class II regulation. Additionally, CcpA seems to be involved in the regulation of genes that do not belong to class I or class II. For most of these genes, the mode of operation is not clear.

When phosphorylated at position Ser-46, HPr can not be phosphorylated at His-15, and therefore can not function as a phosphate donor within the PTS (Deutscher and Saier, 1983). Thus, HPrK/P exerts also an indirect influence on the regulation of PTS mediated sugar uptake.

Many of the genes involved in glycolysis are constitutively expressed. Except for phosphoglucoisomerase and fructose-1,6-bisphosphate aldolase (pgi, fbaA) all glycolytic enzymes are organized in operons (Kunst et al., 1997). The gapA operon includes the glycolytic genes gapA, pgk, tpi, pgm, and eno, coding for glyceraldehyde-3-phosphate dehydrogenase, phosphoglycerate kinase, triose phosphate isomerase, phosphoglycerate mutase, and enolase (see Fig. 1.2) (Kunst et al., 1997; Leyva-Vazquez and Setlow, 1994).

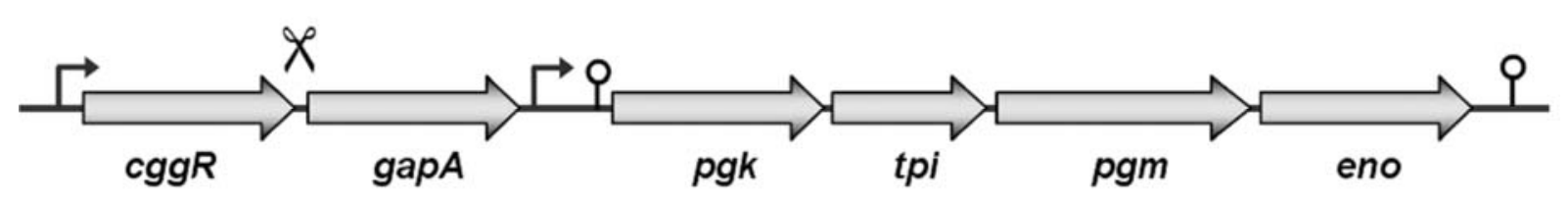

Figure 1.2. Overview on the gapA operon of B. subtilis. The promoter upstream of $\operatorname{cgg} R$ is feedback regulated by CggR. The constitutive promoter upstream of $p g k$ ensures the availability of $p g k, t p i$, $p g m$, and eno under both glycolytic and glyconeogenetic conditions. The processing site between $\operatorname{cgg} R$ and gapA modulates the stability of the polycistronic mRNA. The processed gapA transcript or the processed transcript comprising gapA, pgk, tpi, pgm are more stable compared to the unprocessed mRNAs.

Additionally, the gene coding for the transcription regulatory protein $\mathrm{CggR}$ (central glycolytic gene regulator) is also part of the operon and is located upstream of gapA (see Fig. 1.2) (Fillinger et al., 2000). This regulatory protein represses the gapA operon when no substrate is present to feed glycolysis. The activity of CggR is regulated by the glycolytic intermediate FBP (Doan and Aymerich, 2003; Zorrilla et al., 2007). Additionally, the expression of the gapA operon is also modulated by the availability of amino acids (Ludwig et al., 2001). The gapA operon also seems to be indirectly regulated by CcpA in a class II dependent manner (see above). In a CcpA mutant strain, glucose dependent regulation of gapA is abolished, but no functional cre sequence is present in the promoter region of the operon (Ludwig et al., 2002b). 
The glycolytic enzymes located downstream of gapA are constitutively expressed (Ludwig et al., 2001). This is achieved by a constitutive promoter located between gapA and pgk (see Fig. 1.2). As these enzymes work reversibly (see Fig. 1.1), they are also part of gluconeogenesis. GapA, however, catalyzes exclusively the conversion of glyceraldehyde-3-phosphate to 1,3-bisphosphoglycerate. Thus, it is only part of glycolysis. Besides the direct repression by $\mathrm{CggR}$ and the indirect regulation by CcpA, the gapA mRNA stability is modulated by an endonuclease (see Fig. 1.2) (Ludwig et al., 2001; Meinken et al., 2003).

As mentioned above (see chapter 1.1.1), GapB catalyzes the reverse reaction of GapA, which is the conversion of 1,3-bisphosphoglycerate to glyceraldehyde-3-phosphate. This reaction is part of gluconeogenesis. In contrast to gapA, gapB is repressed in the presence of glucose (Fillinger et al., 2000; Yoshida et al., 2001). Thus, gapA and gapB are also regulated in a reverse manner. More details about the regulation of the gluconeogenetic genes gapB and pckA (see chapter 1.1.1 and Fig. 1.1) have been uncovered recently. In addition to the reversible glycolytic enzymes, both are needed for the efficient formation of glucose-6-phosphate from TCA cycle intermediates. The expression of both genes is repressed in the presence of glucose, but this regulation is independent or, in the case of $p c k A$, only indirectly dependent on CcpA (Blencke et al., 2003; Moreno et al., 2001). Instead, CcpN (control catabolite protein of gluconeogenic genes) was identified to bind in a cooperative manner to the promoter regions of $p c k A$ and $\operatorname{gap} B$, causing transcriptional repression in the presence of glucose (Licht and Brantl, 2006; Servant et al., 2005). In addition, CcpN was shown to bind to the promoter region of $s r 1$, coding for a small regulatory RNA (see also chapter 1.2.2) (Licht et al., 2005).

The genes involved in overflow metabolism ( $p t a, a c k A, a l s S D$ ) and the $p d h A B C D$ operon (see chapter 1.1.1) are also under the control of CcpA. The glucose dependent induction of ackA and pta is directly related to the binding of CcpA to a cre sequence upstream of the respective promoter region (Grundy et al., 1993; Presecan-Siedel et al., 1999; Shin et al., 1999; Turinsky et al., 1998). For the activation of the alsSD operon, CcpA seems to play an indirect role, since no cre sequence could be found in the promoter region (Tobisch et al., 1999b; Turinsky et al., 2000). The direct regulator of the alsSD operon is the transcription activator AlsR, but the exact trigger for its activity is not yet known (Cruz Ramos et al., 2000; Renna et al., 1993). There is not much known about the regulation of the $p d h A B D C$ operon, but there is also indication for an indirect role of CcpA (Blencke et al., 2003). 
The TCA cycle is an important link between carbon- and nitrogen metabolism (see chapter 1.1.1 and Fig. 1.1). Its activity is therefore modulated by the availability of carbon and nitrogen sources in a synergistic manner. Only in the presence of both a good carbon and good nitrogen source the TCA cycle is repressed (Rosenkrantz et al., 1985; Sonenshein, 2002). The main regulation is mediated by $\mathrm{CcpC}$, a transcription regulatory protein of the LysR family (Jourlin-Castelli et al., 2000). It controls the expression of the citZ operon which codes for the citrate synthase, isocitrate dehydrogenase, and malate dehydrogenase (citZ, icd, $m d h$ ). Additionally, icd and $m d h$ contain also CcpC independent promoters (Jin et al., 1996). In addition, the aconitase (citB) is under the control of CcpC (Jourlin-Castelli et al., 2000). It was shown that $\mathrm{CcpC}$ represses the respective genes depending on the availability of glucose as well as glutamine, glutamate, and ammonium. citZ and citB are also subject to CcpA dependent carbon catabolite repression. However, only the promoter region of cit $Z$ contains a CcpA binding site (cre sequence) (Sonenshein, 2002). The influence of CcpA on the regulation of $c i t B$ is an indirect effect resulting from the $C \mathrm{cpA}$ dependent regulation of $c i t Z$ (Kim et al., 2002). CitZ catalyzes the formation of citrate from oxaloacetate and acetyl-CoA. In the presence of glucose, citZ is under CcpA dependent carbon catabolite repression and therefore the intracellular citrate concentration is decreased. Since citrate is the inducer of CcpC repressed genes, the repression of $c i t Z$ by $C c p A$ causes the indirect repression of $c i t B$ (Kim et al., 2003a; Kim et al., 2002). CitB is also repressed in the presence of good nitrogen sources. It was proposed that glutamine or $\alpha$-ketoglutarate is the trigger of this repression, but newer studies indicate that arginine could be the nitrogen signal causing repression of citB (Blencke et al., 2006; Fisher and Magasanik, 1984; Rosenkrantz et al., 1985).

In contrast to the important role of the pentose phosphate pathway for B. subtilis, there is not much known about the regulation of the involved genes. A recent study revealed that the genes encoding the enzymes of this pathway might be constitutively expressed (Blencke et al., 2003).

\subsection{RNA and its role in gene regulation}

The idea that RNAs may serve as regulatory elements was not born just recently. The first were Jacob and Monod who proposed a possible role for RNA in direct gene regulation (Jacob and Monod, 1961). However, when the first regulatory systems were elucidated and it became clear that gene expression was controlled on the level of transcription initiation by 
proteins, they discarded this idea completely. Today, gene regulation by RNA is a field of growing interest and the modes by which RNA regulates gene expression are extremely diverse.

\subsubsection{The structural features of single stranded RNA}

Folding prediction for a protein from its amino acid sequence still remains an unsolved problem. This is due to the large number of 22 codogenic amino acids (when including selenocysteine and pyrolysine) and the fact that folding is not only dependent on sequence but also on the $\mathrm{pH}$ and the salt concentration. Despite the increasing power of computer hardware over the last decade, it is still not possible to predict the right folding structure of even small proteins (Zhang and Skolnick, 2004). Although RNA is made up of only four ribonucleotides, structure prediction is a likewise complicated task. Especially single stranded RNA can adopt secondary structures with a complexity similar to that of proteins. Its structural flexibility is also responsible for its functional diversity (Schroeder et al., 2004). The high flexibility in structure derives from the fact that the four ribonucleotides not only interact by Watson-Crick base pairing to stabilize a secondary structure (Watson and Crick, 1953), but also by so called Hoogsteen, "C-H", and sugar edge interactions. In a Hoogsteen base pairing, the hydrogen in purines at position C-8 instead of position C-2 is involved in hydrogen bond formation. Similarly, the hydrogen in pyrimidines at position C-5 instead of N-3 is involved in "C-H" base pairing interaction. In sugar edge interactions, the hydroxyl group of the ribose interacts with the hydrogen atoms of the RNA bases (see Fig. 1.3). The bases can furthermore interact in either of two different orientations, cis or trans, relative to the hydrogen bonds which altogether results in more than 16 basic types of interaction (see Fig. 1.3) (Leontis et al., 2002; Leontis and Westhof, 2001; Yang et al., 2003). Single stranded RNA can also interact with other RNAs and thereby modulate translation efficiency or the half-life of these molecules.

This flexibility also led to the "RNA world" hypothesis where RNA was responsible for both the maintenance of genetic information and the catalysis of chemical reactions (Gilbert, 1986). In an ancient world where neither DNA nor proteins have existed, RNA could have catalyzed its own replication and this error-prone process might have favored a quick evolution of life (Jeffares et al., 1998; Johnston et al., 2001; Long et al., 2003). Today, four billion years after the evolution of life started, there are still some hints present in modern organisms indicating RNA as a multifunctional molecule apart from being a messenger for genetic information. 

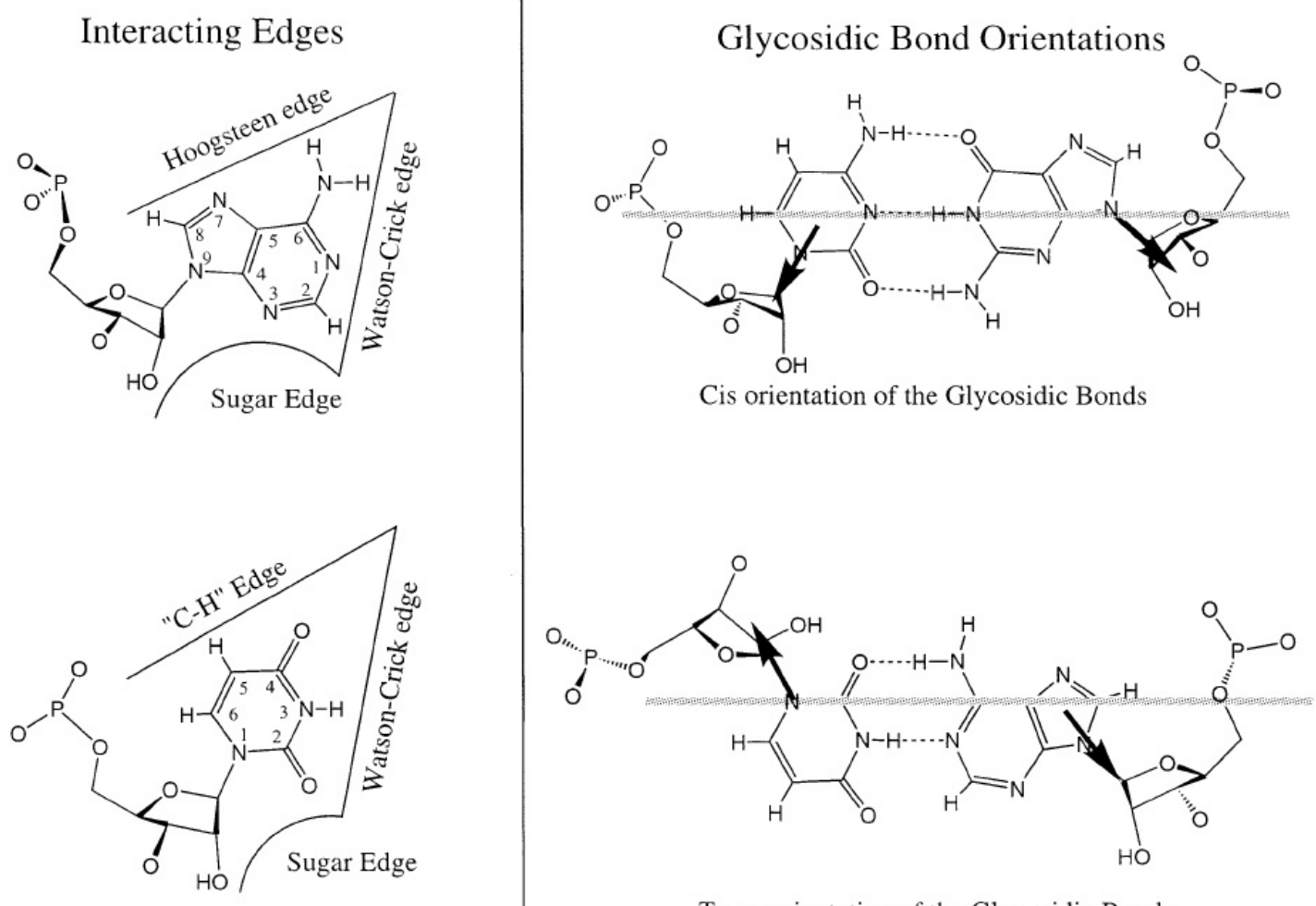

Trans orientation of the Glycosidic Bonds

Figure 1.3. Left: Illustration of the possible interaction sites of the RNA bases (Watson-Crick, Hoogsteen, C-H, and sugar edge). Right: Illustration of possible orientations of the glycosidic bonds in a Watson-Crick base pair interaction. The upper picture shows an uracile-guanine interaction with glycosidic bonds in cis orientation. The lower picture shows an uracile-adenine interaction with trans orientation of the glycosidic bonds (Leontis et al., 2002).

In some viruses, RNA is still the long term storage of genetic information (Domingo and Holland, 1997; Strauss and Strauss, 1988) and the process of DNA replication can not be initiated without an RNA primer (Eigen et al., 1981). Additionally, many ribozymes, like the spliceosome and the ribosome, are evidence of the catalytic power of RNA. In these complexes the RNA exhibits the catalytic function and the protein part is mostly responsible for giving the proper shape to the RNA catalytic center (Noller, 1993; Wise, 1993). Finally, the fact that ribonucleotides serve as precursors for deoxynucleotide synthesis points to the important role that RNA carried out prior to DNA in the history of life (Joyce, 1989).

Another important characteristic of RNA is its much lower half-life as compared to DNA. This is due to the hydroxyl group at the 2'-C atom of the ribose. Thus, an RNA polymer is susceptible to spontaneous hydrolysis leading to chain cleavage (Winkler and Breaker, 2003). As a result, RNA is also unstable against acidic or alkaline hydrolysis (Larralde et al., 1995). However, exactly this feature makes RNA so catalytically reactive and thus broadly applicable. 
The catalytic power of RNA is even more impressive when considering that RNA is not only made up of just four different components, but that these are also very similar in size and chemistry. Even proteins, although having access to an arsenal of amino acids that vary significantly in size, charge, and polarity, can not fulfill their job without the use of lower molecular weight cofactors (Jadhav and Yarus, 2002; Reichow and Varani, 2006). Therefore, the affinity of some RNAs to lower molecular weight agents like CoA, cyanocobalamin, and biotin might be inherited from the RNA-world, where RNA needed the help of this molecules to exert its catalytic functions (Jadhav and Yarus, 2002).

As there are more complete genome sequences published every year, comparative sequence analysis of RNAs that might exhibit catalytic or regulatory functions seems to be feasible very easily. However, not only the actual sequence, but also the structural features of catalytic RNAs are important. For further research, the accurate structure prediction becomes essential for the RNA science community. There are elaborated algorithms for RNA secondary structure prediction. A very common tool for this purpose is "Mfold" (Mathews et al., 1999; Zuker, 2003). The software includes Watson-Crick base pairing to calculate the secondary structure of an RNA sequence at a given temperature. A viable method to verify the correctness of in silico predicted structures is the introduction of mutations. If the structure prediction is accurate, both mutations that are tolerated and mutations that destroy the secondary structure and consequently the activity of the catalytic RNA can be predicted and tested (Barash, 2003). The programs for RNA structure prediction become also more accurate by incorporating more sophisticated algorithms and combining them with data obtained from high-resolution RNA structures (Gautheret and Gutell, 1997; Lillo et al., 2002; Mathews et al., 2004; Sykes and Levitt, 2005). Thus, it is very likely that with progressive and optimized in silico methods by far more catalytic and regulatory RNAs will be found in all sequenced organisms.

\subsubsection{Non-coding RNAs}

It was first discovered in bacteria that small non-coding RNA molecules (ncRNA) had a negative effect on the expression of other genes (Mizuno et al., 1984; Tomizawa et al., 1981). The replication control of a plasmid (ColE1) and the transcription of an Escherichia coli outer membrane protein $(o m p F)$ were found to be under the control of small RNA transcripts. Later on, small RNA molecules were used to influence gene regulation in eukaryotes. These

artificially designed RNA molecules were complementary to dedicated mRNA target sequences, and were therefore called "antisense RNAs" (asRNA). Although the mechanism 
was not fully understood, it became a useful tool for influencing gene expression in organisms that are not fully accessible to genetic manipulations (Aigner, 2006; Amarzguioui et al., 2005).

In eukaryotes, many types of small RNAs have influence on transcription and stability of different kinds of RNAs. Micro RNAs (miRNAs) play a role in the silencing of coding genes, control chromosome organisation, inhibit viruses and transposable elements, and have influence on proliferation, apoptosis, and differentiation (Ambros, 2004; Aravin and Tuschl, 2005; Xu et al., 2004; Ying et al., 2006). Small nuclear RNAs (snRNAs), small nucleolar RNAs (snoRNAs), and small guide RNAs (gRNAs) are involved in mRNA and rRNA processing (Carthew, 2006; Mattick and Makunin, 2005; Stuart and Panigrahi, 2002; Valadkhan, 2005; Wagner and Flärdh, 2002).

Although bacterial ncRNAs, which are also called small RNAs (sRNAs) (Gottesman, 2004), play a similar role in gene regulation as eukaryotic miRNAs, they differ in many aspects significantly from each other. While their eukaryotic counterparts are processed from double stranded regions of stem loop structures, bacterial ncRNAs are single stranded transcripts. Processed eukaryotic miRNAs also feature a size of about 21 to 23 nucleotides (Sewer et al., 2005). In contrast, bacterial ncRNAs are not processed and are generally 80-100 bps in size (Gottesman, 2004; Gottesman, 2005). They fold into stem-loop secondary structures which help to stabilize the molecules and prevent degradation (Massé et al., 2003). Although protein cofactors play a crucial role for the activity of regulatory RNAs in both kinds of organisms, the mode of regulation differs. In bacteria, a protein factor does not seem to be necessary in all cases, but for some ncRNAs the protein Hfq is important for their regulatory activity (Valentin-Hansen et al., 2004; Zhang et al., 2003). Hfq, first discovered in E. coli, seems to be necessary for the structural reorganisation of the interacting RNA molecules, and thus mediates binding (Møller et al., 2002). More than 20 ncRNAs are known to directly interact with Hfq (Zhang et al., 2003). For the degradation process of the target mRNA, RNase E seems to play a crucial role (Moll et al., 2003).

The regulatory RNAs can cause inhibition of translation and RNA degradation, but in contrast to miRNAs and siRNAs, also stimulation of translation and mRNA stabilisation was observed (Majdalani et al., 1998; Massé et al., 2003; Opdyke et al., 2004; Repoila and Gottesman, 2003). Bacterial non-coding RNAs are involved in the regulation of virulence, stress response, quorum sensing, uptake systems, and plasmid replication (Brantl, 2002; Guillier et al., 2006; Lenz et al., 2004; Massé et al., 2003; Massé et al., 2005; Repoila and Gottesman, 2003; Romby et al., 2006). 
In contrast to E. coli, there is not much known about ncRNAs in B. subtilis. Recently, it was found that the small regulatory RNA $s r l$ is involved in the fine tuning of arginine catabolism (see chapter 1.1.2) (Heidrich et al., 2006). Another small regulatory RNA (ratA) was found to act as an antitoxin (Silvaggi et al., 2005). Although YmaH was identified as an Hfq analog in B. subtilis it does not seem to be involved in $s r 1$ and ratA mediated regulation (Heidrich et al., 2006; Silvaggi et al., 2005). A recent study also revealed that ncRNAs take part in the complex regulatory process of sporulation in B. subtilis (Silvaggi et al., 2006).

\subsubsection{RNA switches}

The ability of single stranded RNA to adopt complex three-dimensional structures is essential for gene regulation mediated by RNA switches (riboswitches). The underlying principle is the possibility of an RNA sequence to fold into two distinct alternative structures (Mironov et al., 2002). This structural change is mediated by the presence of an external effector. The "switch" can be turned on or off by a lower molecular agent, a protein, another RNA molecule, or even in response to higher or lower temperature (Grundy and Henkin, 1993; Narberhaus, 2002; Stülke, 2002; Winkler, 2005). Thus, RNA-switches are cis acting elements that control gene regulation by folding into mutually exclusive structures.

Functional RNA-switching elements are composed of two domains: The first domain is a ligand-binding domain that serves as a natural aptamer, and the second one serves as a genetic control element also named expression platform. The genetic control element senses the status of the binding domain and exerts genetic control onto the subsequent coding genes (Winkler and Breaker, 2003). Therefore, RNA-switching elements are generally located in the 5 '-untranslated region of their regulated genes.

The ligand-binding sites of RNA switches bind their ligands very efficiently and independently from the presence of the genetic control sequence. Thereby, they are highly selective and discriminate between closely related structures (Nudler, 2006; Reichow and Varani, 2006). This selectivity is mediated by a mix of standard and non standard base pairs, hydrogen bonds, van der Waals, and electrostatic and stacking interactions (Nudler, 2006).

The regulation by the RNA genetic control elements can occur on the level of transcription and translation (Nudler and Mironov, 2004; Vitreschak et al., 2002). In the first case, the transcription of a coding gene is prematurely stopped by an intrinsic terminator. The formation or the suppression of the terminator structure is dependent on the binding of a certain ligand to the ligand-binding domain. 
Transcription termination

(a)
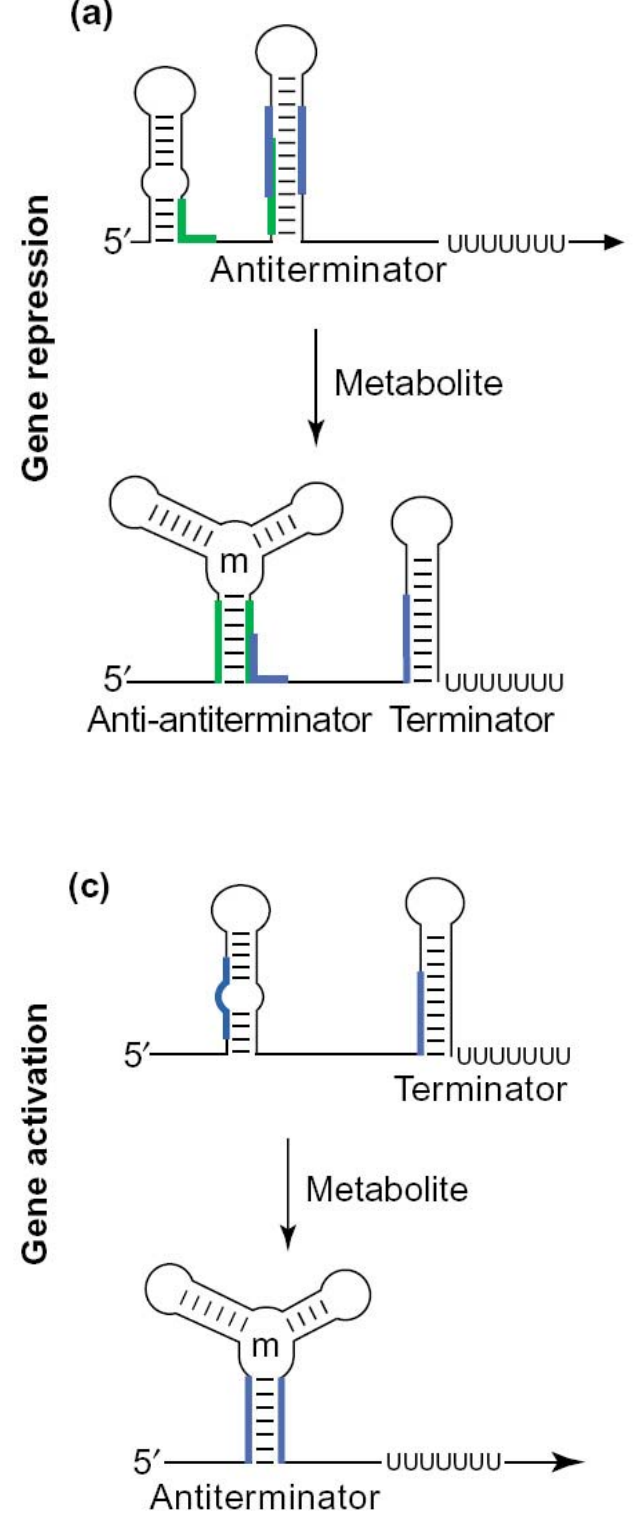

Translation initiation

(b)
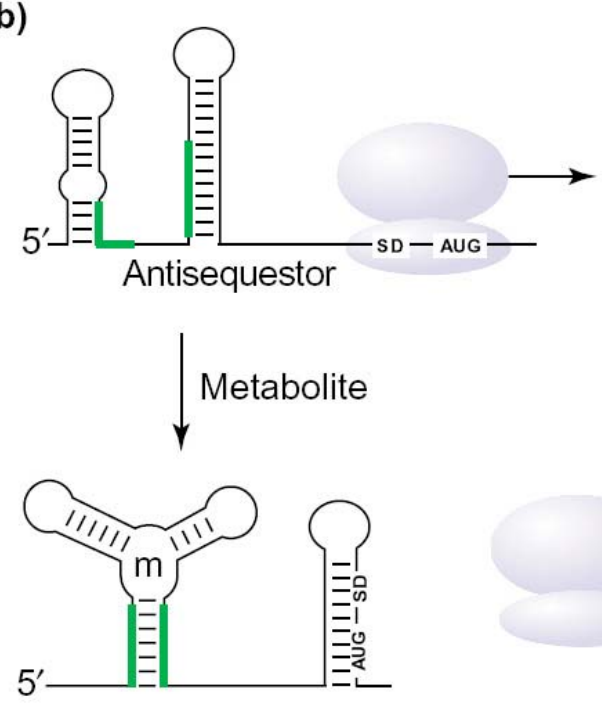

Anti-antisequestor Sequestor

(d)
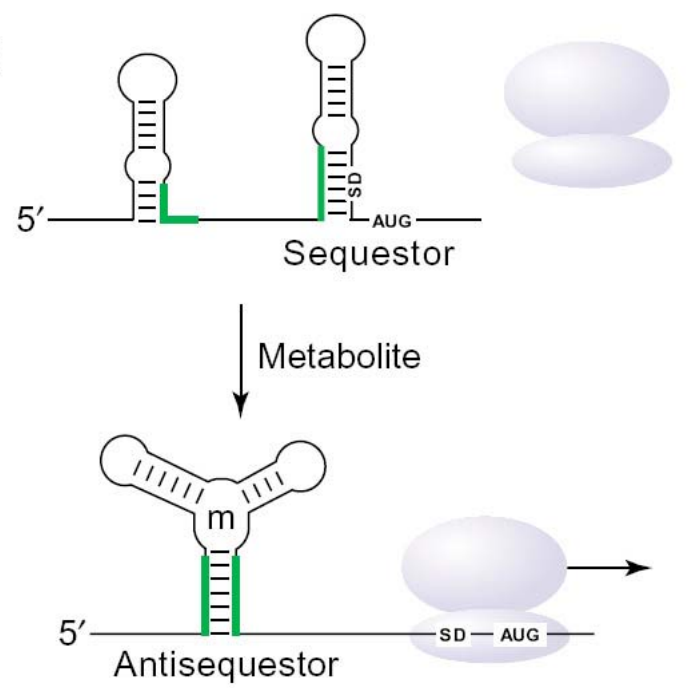

Figure 1.4. Possible regulation modes of RNA switches. Left $(a, c)$ : Regulation by transcription termination. The binding of a metabolite causes the formation of a terminator which leads to transcription termination (a), or metabolite binding represses the formation of a stable terminator and enables readthrough into the coding genes (c).

Right (b, d): Regulation by translation initiation. Metabolite binding causes formation of an RNA structure (sequestor) that comprises Shine Dalgarno sequence and start codon which inhibits translation initiation (b). Metabolite binding favors a secondary structure that comprises the part of the sequestor sequence. This represses the structure of the sequestor and gives access to Shine Dalgarno sequence and start codon (d) (Nudler and Mironov, 2004). 
The binding of the ligand can theoretically result in repression or activation of the coding gene dependent on the overall architecture of the RNA switch (see Fig. 1.4). As for the second case, regulation occurs when the whole mRNA is already synthesized. The RNA switch controls the accessibility of the Shine Dalgarno (SD) sequence for the ribosomes and thus controls gene regulation on the level of translation initiation (sequestration) (Nudler and Mironov, 2004; Winkler, 2005; Winkler and Breaker, 2003). Examples for both possibilities have already been found in different organisms. Whether the RNA switch represses or activates gene translation upon binding of the ligand, as mentioned above, depends also on the overall design of the riboswitch (see Fig. 1.4). However, most of the known regulatory elements that act on the level of translation initiation repress gene regulation upon ligand binding. There are also known cases where the mRNA is processed upon ligand binding and therefore, these RNA switches can be considered as metabolite dependent ribozymes (Jansen et al., 2006; Kubodera et al., 2003; Sudarsan et al., 2003a; Winkler et al., 2004).

It is possible that RNA switches are actually not able to "switch" their conformation back and forth in response to ligand availability. Perhaps the decision which structure to adopt is only made once in the lifetime of such an RNA molecule. The differentiated structure folding is a time critical process and occurs during transcription elongation depending on the availability of the ligand (Nudler, 2006). Thus, riboswitch folding might be a one way street.

Among bacteria, E. coli and B. subtilis are the best studied organisms. This also holds true for the research on gene regulation by RNA switches. It is notable, that riboswitches found in E. coli are mostly regulating on the level of translation initiation whereas riboswitches found in B. subtilis are generally regulating on the level of transcription termination (Nudler and Mironov, 2004).

The number of genes controlled by regulatory RNA elements is hard to evaluate. In 2003 , the number of genes that were known to be regulated by riboswitches was $2 \%$ of the total genome of B. subtilis (Winkler and Breaker, 2003). Two years later the number of genes known to be regulated in that way has increased to $4.1 \%$ (Winkler, 2005). Thus, gene regulation by riboswitches seems to be common in bacteria (Barrick et al., 2004). These cis acting regulatory RNA elements have been found in many other bacteria (Miranda-Rios et al., 2001; Rodionov et al., 2002; Vitreschak et al., 2002), archaea (Rodionov et al., 2002), and even in eukaryotes like fungi and plants (Sudarsan et al., 2003a; Thore et al., 2006), whereas in humans only one example has been found so far (Bugala et al., 2005).

In all domains of life, the ligand-binding domain of a particular RNA switch is highly conserved, whereas the regulatory element may vary. For example, the thiamine 
pyrophosphate binding riboswitch is found in Gram-negative and Gram-positive bacteria as well as in eukaryotes. Although the ligand binding domain is highly conserved among all these organisms (Serganov et al., 2006; Thore et al., 2006), the expression platform does not only vary in sequence but even differs in the mode of genetic control (Kubodera et al., 2003; Mironov et al., 2002; Winkler et al., 2002b). In Gram-positive bacteria, gene regulation is mediated by control of premature transcription termination while in Gram-negative bacteria the genes are controlled by translation initiation. In eukaryotes however, the riboswitch acts as a modulator of splicing. There are also cases known where different genes of the same metabolic pathway are regulated differently in the same organism. The ypaA gene of the Bacillus and Clostridium group is regulated both at the level of transcription and translation (Lee et al., 2001; Winkler et al., 2002a).

Most of the metabolite binding riboswitches are widespread among evolutionary distant organisms. Considering this together with the high conservation of the ligand binding sites of these regulators could lead to the assumption that riboswitches are of ancient origin and relics of the RNA world (Joyce, 2002) (see chapter 1.2.1). The particular metabolite binding sites were optimized throughout evolution and thus show this high similarities among distant organisms. The fact that no additional factors like proteins are involved also supports the theory of an ancient origin of riboswitches (Vitreschak et al., 2004; Winkler and Breaker, 2003). However, the same argument can also be quoted to suggest that RNA switches have been spread by numerous horizontal gene transfers. The lack of necessity for any additional factor makes the transfer from one organism to another a comparatively easy task.

Most of these RNA control elements have been found by comparative in silico analyses and are 70 to 200 bases in size. Recently, an RNA switch has been found whose functional region is only 35 bases in size (Winkler, 2005). This indicates the possibility that much more RNA regulatory elements will be found with more precise and better adapted algorithms.

\subsubsection{Regulation by RNA thermometers}

RNA thermometers are RNA structures that are located in the 5'-untranslated region (UTR) of the mRNA of a coding gene, but in contrast to all other RNA switches they are capable of regulating genes without the need of any additional factors (Lai, 2003; Narberhaus, 2002; Narberhaus et al., 2006). Instead of sensing the concentration of molecules, RNA thermometers respond to a physical signal, i.e. temperature. Like other RNA switches, the sequence in the 5'-UTR of an RNA thermometer can adopt two distinct structures. One 
structure blocks the Shine Dalgarno sequence and usually also the start codon by intramolecular base pairing, whereas the second structure allows access of the ribosome. The alteration of the RNA structure depends on a temperature shift that can be in some cases as small as $8^{\circ} \mathrm{C}$.

A conserved RNA element called ROSE ( $\underline{R}$ epression $\underline{O f}$ heat- $\underline{S}$ hock gene $\underline{E}$ xpression) in the 5'-UTR of multiple heat shock genes of Bradyrhizobium japonicum was found to control genes by sensing temperature (Münchbach et al., 1999; Narberhaus et al., 1998; Narberhaus et al., 1996). In the 5'-leader of $h s p A$, ROSE represses expression at temperatures of $30{ }^{\circ} \mathrm{C}$ and below, but deletion of this region abolished regulation and resulted in constitutive expression. The ROSE RNA thermometer does also control heat-shock genes in other Rhizobiales and many $\alpha$ - and $\gamma$-proteobacteria (Nocker et al., 2001; Waldminghaus et al., 2005). Some of these genes are under dual temperature regulation, as they are also controlled by a $\sigma^{32}$ dependent promoter.

Not only heat but also cold is a physical condition that can be sensed by RNA structures. Analysis of the $\operatorname{csp} A$ mRNA, coding for a cold shock gene in E. coli, revealed that this gene is controlled by alternative RNA structures in the 5'-leader region of its mRNA. In this case, accessibility for ribosome binding is possible at low temperatures, whereas higher temperatures block translation of this mRNA (Yamanaka et al., 1999). Additionally, rapid RNaseE degradation of the $\operatorname{csp} A$ mRNA occurs at higher temperatures (Fang et al., 1997). It is notable that all RNA thermometers found so far are regulating gene expression rather on the level of translation than transcription.

\subsubsection{RNA mediated regulation}

Gene regulation that depends on specific RNA-RNA interactions was already discussed in chapter 1.2.2. Here, only gene regulation where a second RNA molecule switches the folding status of an RNA dependent riboswitch will be described.

In many Gram-positive bacteria a conserved structural RNA element called T-box can be found in the leader region of many aminoacyl-tRNA synthetases, and genes of amino acid biosynthesis and amino acid transport (Gerdeman et al., 2003; Grundy and Henkin, 1993; Grundy and Henkin, 2003). The T-box regulates genes by an intrinsic terminator causing premature transcription termination. However, a conserved sequence in the 5'-region of the controlled mRNAs can directly interact with the appropriate uncharged tRNA. If, for example, the intramolecular concentration of glycine gets low, the ratio of charged to uncharged tRNA ${ }^{\text {Gly }}$ changes towards the latter. The intermolecular base pairings between the 
uncharged tRNA and the T-box mRNA stabilizes an antiterminator structure, which leads to the transcription of the downstream genes (Grundy et al., 2002; Putzer et al., 2002). The RNA-RNA interaction is specific for the T-box RNA of a certain gene and the associated uncharged tRNA. This specificity is mainly mediated by two points: First, a specifier sequence in the leader of the mRNA forms base pairing interactions with the tRNA anticodon sequence (Grundy and Henkin, 2003; Grundy et al., 1997). Second, the overall structure of the tRNA is recognized by the leader RNA (Grundy et al., 2000; Yousef et al., 2003). Additionally, the 3'-terminal region of the tRNA pairs with the mRNA and stabilizes a conformation that promotes expression of the coding genes (Grundy et al., 1994). These interactions work in a complex 3D-structural manner and there is also evidence that other factors apart from base specific interactions play a role in target recognition (Fauzi et al., 2005). Genes that are regulated by a tRNA-binding riboswitch are best studied in B. subtilis. Among others, the tRNA synthetase genes cysES (cysteine), leuS (leucine), pheS (phenylalanine), thrS, thrZ (threonine), glyQS (glycine), and the ilv-leu-operon (Condon et al., 1996; Grundy and Henkin, 2004) are regulated by the T-box riboswitches.

\subsubsection{Metabolite mediated regulation}

In addition to RNA thermometers, the metabolite binding riboswitches might represent the most ancient form of RNA switches, and probably even the oldest form of gene regulation (Vitreschak et al., 2004). Without the need of any additional protein factors, regulation is mediated by direct sensing the concentration of metabolites, e.g. the product of the regulated pathway (Henkin and Yanofsky, 2002). Among the best-studied metabolite binding riboswitches are those that regulate metabolic pathways of vitamin, amino acid, and purine biosynthesis.

The thi-box (Miranda-Rios et al., 1997; Miranda-Rios et al., 2001) is an RNA sequence of 39 nucleotides which is able to bind thiamine pyrophosphate (TPP). It is highly specific for TPP binding, as it binds neither its precursor thiamine (vitamine B1) nor thiamine monophosphate (Mironov et al., 2002; Winkler et al., 2002b). This regulatory element is very well documented in E. coli, Rhizobium etli, and B. subtilis (Begley et al., 1999; Petersen and Downs, 1997) and has been found in over 100 other bacteria (Rodionov et al., 2002; Sudarsan et al., 2003a). Furthermore, it has been found in archaea, fungi, and plants. Thus, the thielement is the most widespread riboswitch found so far. It is involved in the regulation of many genes of thiamine biosynthesis and transport. The ligand binding domain of this riboswitch has been found to interact with the negatively charged phosphate groups of TPP 
(Serganov et al., 2006; Thore et al., 2006). As RNA itself is a negatively charged molecule, this was unexpected. Interestingly, the structures of TPP binding riboswitches from the different organisms are almost identical, while the mode of regulation can vary between transcriptional or translational level or can even occur by modulating mRNA splicing (Kubodera et al., 2003; Sudarsan et al., 2003a; Winkler et al., 2004).

Another riboswitch involved in the biosynthesis of a vitamin is the $r f n$ element (Gelfand et al., 1999; Vitreschak et al., 2002; Winkler et al., 2002a). It has been found in more than 40 different bacterial groups and controls genes involved in the biosynthesis or transport of riboflavin (vitamin $\mathrm{B}_{2}$ ), a precursor of flavin mononucleotide (FMN) and flavin adenine dinucleotide (FAD). It is best studied in B. subtilis where the $r f n$ element is controlling the ribDEAHT operon by directly sensing the concentration of FMN (Kil et al., 1992; Mironov et al., 1994). The affinity to FMN is 100 -fold stronger than to riboflavin, which is chemically almost identical but lacks an additional phosphate group (Winkler et al., 2002a). The ypaA gene of B. subtilis, coding for a riboflavin transporter, is regulated at the level of translation as binding of FMN blocks the accessibility of the SD sequence for the ribosomes (Winkler et al., 2002a).

Some genes coding for amino acid synthetic operons are also controlled by riboswitch mechanisms. The S-box is a conserved riboswitch element involved in sulfur metabolism and the synthesis of the sulfur containing amino acids methionine and cysteine (Grundy and Henkin, 1998). This regulatory element has been almost exclusively found in Gram-positive bacteria and is best studied in B. subtilis (Grundy and Henkin, 2003). The regulation of these genes is dependent on the availability of S-adenosylmethionine (SAM), an essential coenzyme directly synthesized from methionine (Grundy and Henkin, 1998; Winkler et al., 2003).

The lys-element is part of another riboswitch controlling amino acid synthetic genes. Like the S-box, it has been found mainly in Gram-positive bacteria. In B. subtilis, it controls the lys $C$ gene coding for the first enzyme of lysine biosynthesis (Kochhar and Paulus, 1996; Mandal et al., 2003). The binding domain of this RNA switch is able to discriminate between L- and D-lysine and also rejects binding of ornithine, homolysine, and 5-hydroxylysine which all differ very slightly from each other (Sudarsan et al., 2003b). However, the compound aminoethylcysteine (AEC), which is an analog of L-lysine, is bound by this riboswitch. This molecule is toxic to $B$. subtilis cells and this could be due to the repression of lysine synthetic genes by premature transcription termination caused by the "wrong" ligand (Lu et al., 1992; Winkler and Breaker, 2005). 
A glycine dependent riboswitch has been found in a variety of bacteria (Barrick et al., 2004). The conserved element of this riboswitch is called gcvT-motif and has been studied in B. subtilis and Vibrio cholerae (Mandal et al., 2004). In B. subtilis, this RNA-motif is involved in regulation of a glycine catabolic operon $(g c v T-g c v P A-g c v P B)$ which is necessary to use glycine as an energy source. Interestingly, this riboswitch element has a tandem aptamer configuration with a 1:1 stoichiometry between glycine and each individual aptamer (Mandal et al., 2004). By binding of a single glycin molecule to one of the two aptamer sites, the glycine binding affinity of the second site is improved by 1000-fold. Thus, this data indicates a cooperative binding mode of the riboswitch resulting in an almost "digital" regulation of the controlled operon.

Riboswitches that control purine synthetic operons are also best studied in B. subtilis. Genes involved in guanine or adenine metabolism are controlled by a conserved RNA switching element (G-box) (Mandal et al., 2003; Mandal and Breaker, 2004). This regulatory element directly binds guanine (G-switch RNA) or adenine (A-switch RNA) specifically and although both compounds are very similar to each other, no cross-talk has been observed. The pur operon, consisting of 12 genes coding for de novo synthesis of inosine monophosphate (IMP), and the $x p t-p b u X$ operon, encoding xanthine phosphoribosyltransferase and a xanthin transporter, are regulated by the G-box riboswitch (Mandal et al., 2003). The conserved G-box element has also been found in the leader region of three other operons: $p b u G$, nup $G$ and $p b u E$ which are purine nucleoside transport proteins. The riboswitch of the $p b u E$ operon, coding for a purine efflux pump, binds adenine rather than guanine and upon binding of the ligand, gene expression is activated. The difference in ligand specificity is mediated by a single base pair exchange in the ligand-binding domain of these RNA switches. This single base exchange transforms a guanine-binding switch into an adenine-binding one (Lescoute and Westhof, 2005; Mandal and Breaker, 2004; Noeske et al., 2005).

The $g \operatorname{lm} S$-riboswitch is mainly found in Gram-positive bacteria where it regulates expression of the GlmS protein (Winkler et al., 2004), which catalyzes the conversion of fructose-6-phosphate to glucosamine-6-phosphate (GlcN6P). The glmS-riboswitch has a high affinity to GlcN6P and regulates this gene by cleavage of the $g \operatorname{lm} S$-mRNA upon binding of its ligand. Thus, the $g \operatorname{lm} S$-riboswitch acts like a GlcN6P dependent ribozyme and is therefore also called $g \operatorname{lm} S$-ribozyme (Winkler et al., 2004). Newer studies indicate that the $g l m S$ cleavage site is very close to the ligand binding domain of the riboswitch and GlcN6P could have a coenzyme-like role in the catalytic core of this ribozyme (Jansen et al., 2006). 


\subsubsection{Protein mediated regulation}

Protein mediated regulation of an RNA switch can be divided into two subclasses. The first class works similar to the metabolite binding riboswitches but instead of sensing the concentration of a small molecule it measures the concentration of a certain protein. In most cases, the protein is the product of a feedback-regulated pathway. A good example for this class is the feedback repression of some ribosomal proteins (r-proteins) (Zengel and Lindahl, 1994). These proteins are normally associated with ribosomal RNAs, but if synthesized in excess, they bind to the 5'-UTR of their coding gene and negatively affect translation (Springer et al., 1997). There is indication that the leader sequence of the regulated gene mimics the structure of the rRNA r-protein binding site (Merianos et al., 2004; Nomura et al., 1980; Stelzl et al., 2003). This negative feedback regulation ensures that no energy is wasted for r-protein synthesis if its concentration is already in excess to ribosomal RNA.

The second class of protein mediated RNA switches differs from the first as the proteins are specifically designed to regulate gene expression by RNA binding. For an additional level of regulation, these proteins are not just sensed by their concentration but are also regulated by their binding activity. Their binding activity can be regulated either by lower molecular weight factors, by another regulatory protein, or by protein modification events, such as phosphorylation (see also chapter 1.3). As they control transcription termination, they are named terminator or antiterminator proteins, depending on their mode of operation.

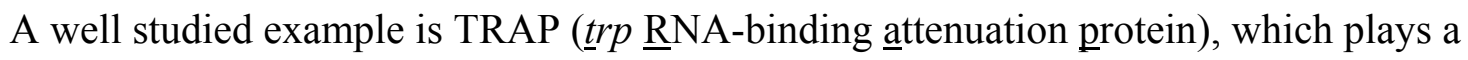
role in regulation of tryptophan biosynthesis in B. subtilis (Babitzke, 2004; Gollnick, 1994; Otridge and Gollnick, 1993). TRAP is composed of 11 identical subunits that form a ring-like structure and it controls expression of the trpEDCFBA operon and of trpG. The leader sequence of this operon is wrapped around the protein, which leads to transcription termination. TRAP binds to a stretch of $11(\mathrm{G} / \mathrm{U}) \mathrm{AG}$ repeats in the leader sequence (Antson et al., 1999; Antson et al., 1995; Gollnick et al., 1995). Additionally, it negatively regulates translation of some transport and efflux genes by blocking ribosomal binding to the mRNA (Babitzke and Gollnick, 2001; Yakhnin et al., 2006; Yakhnin et al., 2004). Activity of TRAP is activated by tryptophan binding. One molecule of tryptophan binds to each TRAP subunit in a cooperative manner. Its activity is negatively regulated when binding to anti-TRAP (AT) which is expressed when the concentration of uncharged tRNA ${ }^{\operatorname{trp}}$ is high (Sarsero et al., 2000; Shevtsov et al., 2005; Valbuzzi and Yanofsky, 2001). Therefore, TRAP activity is regulated by a lower molecular weight factor, i.e. tryptophan, as well as by a regulatory protein. Gene 
regulation by TRAP is highly nuanced in a way that would not be possible just with a tryptophan-dependent riboswitch.

Another example of an RNA switch controlling protein whose activity is regulated by a lower molecular weight factor is PyrR. In contrast to the purine-binding riboswitches (see chapter 1.2.3.3), the pyrimidine concentration is not directly sensed by a regulatory RNA. Instead, the PyrR protein binds as a dimer to the leader sequences in the pyr-operon coding for pyrimidine biosynthetic genes (Quinn et al., 1991). The binding sites are located in the 5'-UTR and within the pyrR-pyrP and pyrP-pyrB intercistronic regions of this operon (Grabner and Switzer, 2003; Zhang and Switzer, 2003). Without binding of PyrR, an antiterminator structure is formed, enabling transcription. Binding of active PyrR to its target sequence forms and stabilizes an anti-antiterminator structure, which prevents antiterminator formation. Under these conditions a terminator structure leads to transcription termination (Switzer et al., 1999). Activity of PyrR is dependent on the availability of pyrimidines. If the intracellular concentration of UMP and UTP is high, PyrR is active and binds as a dimer to its target sequence. In Bacillus caldolyticus GMP was found to bind to PyrR and antagonizing its RNA binding ability (Chander et al., 2005). As PyrR itself has uracil phosphoribosyltransferase activity it can be assumed that it was not originally designed to bind RNA structures. Therefore, this might be a good example of how a protein can evolve to exert new functionality and how the RNA coevolved in that case (Tomchick et al., 1998).

There are many more examples of RNA-binding proteins, which modulate RNA-structure upon binding, e.g. HutP (Kumar et al., 2006; Oda et al., 2000; Wray and Fisher, 1994), GlpP (Rutberg, 1997), AmiR (Wilson and Drew, 1995; Wilson et al., 1993), NasR (Chai and Stewart, 1998; Chai and Stewart, 1999), and BglG (Amster-Choder and Wright, 1992; Mahadevan and Wright, 1987; Schnetz and Rak, 1990). They can be found in many different kinds of bacteria. Antiterminator proteins that are controlled by reversible phosphorylation events, like the BglG family of antiterminator proteins, are discussed in chapter 1.3 .

\subsubsection{RNA aptamers: control by artificial regulatory RNA elements}

Genetic control elements, like the lac-operon regulatory system (Kercher et al., 1997), are common tools for controllable expression of gene constructs. Natural riboswitches are able to regulate gene expression without the need of any additional protein factors and could therefore expand the repertoire of genetic control elements with a more general applicable system. There was not much known about these natural regulatory elements when some 
scientists started to make use of the specific binding affinity that RNA provides. With an in vitro selection method called SELEX (Ellington and Szostak, 1990) they were able to screen for RNA sequences (aptamers) that specifically bind to small organic molecules. Even small proteins can bind to RNAs selected by this method (Hermann and Patel, 2000). With lessons learned from natural RNA switches it was possible to engineer artificial but functional RNA-elements capable of switching gene expression dependent on ligand binding (Werstuck and Green, 1998).

A good example is the Tet-repressor system (Orth et al., 2000; Saenger et al., 2000). This system derived from Escherichia coli where it controls tetracycline dependent gene expression. TetR binds to the tet-operator if no tetracycline is available and thereby blocks transcription. If the antibiotic is present, it binds TetR and induces a structural change that releases the protein from the DNA. The system is used as a genetic switch to control gene expression in bacteria and eukaryotes. However, this system has some limitations in eukaryotes. Therefore, a system was engineered to control expression without the need of a protein factor. A tetracycline-binding aptamer, found by SELEX, was placed in the 5'-UTR of a reporter gene and this construct was functional in controlling gene expression in yeast dependent on the availability of tetracycline. If the antibiotic is available, it binds to the RNA and interferes with its accessibility for the ribosomes (Hanson et al., 2005; Müller et al., 2006; Suess et al., 2003).

Another example is a synthetic theophylline responsive riboswitch. A theophylline binding aptamer was combined with a translational control element that exerts regulation. Normally, translation is blocked but binding of theophylline causes a minor structural reorganisation of the control element, which leads to efficient translation (Desai and Gallivan, 2004; Suess et al., 2004).

Aptamers are also used as biosensor elements for molecular analysis ranging from small molecules to proteins (Hesselberth et al., 2003; Seetharaman et al., 2001). However, the binding affinity of these artificially isolated RNA molecules to their targets is far from that observed for natural riboswitches (Nudler, 2006). 


\subsection{PTS-controlled antitermination in B. subtilis}

\subsubsection{The PTS and its role in sugar transport}

The phosphoenolpyruvate:sugar phosphotransferase system (PTS) plays a central role in the regulation of catabolic operons in many bacteria (Meadow et al., 1990; Postma et al., 1993; Reizer et al., 1988). As mentioned above (see chapter 1.1.1), the PTS consists of general and sugar specific components. The sugar specific enzymes (enzyme II or EII) consist of three or four domains. These domains can be separate proteins or fused together forming single polypeptides (Postma et al., 1993). The general proteins are the cytoplasmatic proteins enzyme I (EI) and HPr. EI transfers phosphate from phosphoenolpyruvate (PEP) to HPr. HPr features two phosphorylation sites. The phosphate coming from EI is transferred to position His-15 of HPr, and then passed to a sugar specific EII. When the relevant sugar is available, it is phosphorylated by EIIBA, whereas EIIC is involved in the uptake process (see Fig 1.5). Thus, the membrane spanning sugar permeases accomplish sugar uptake paired with simultaneous phosphorylation. The phosphate derived from PEP is transferred from phosphoenolpyruvate to the sugar via EI, HPr (His15), and EII (see Fig. 1.5).

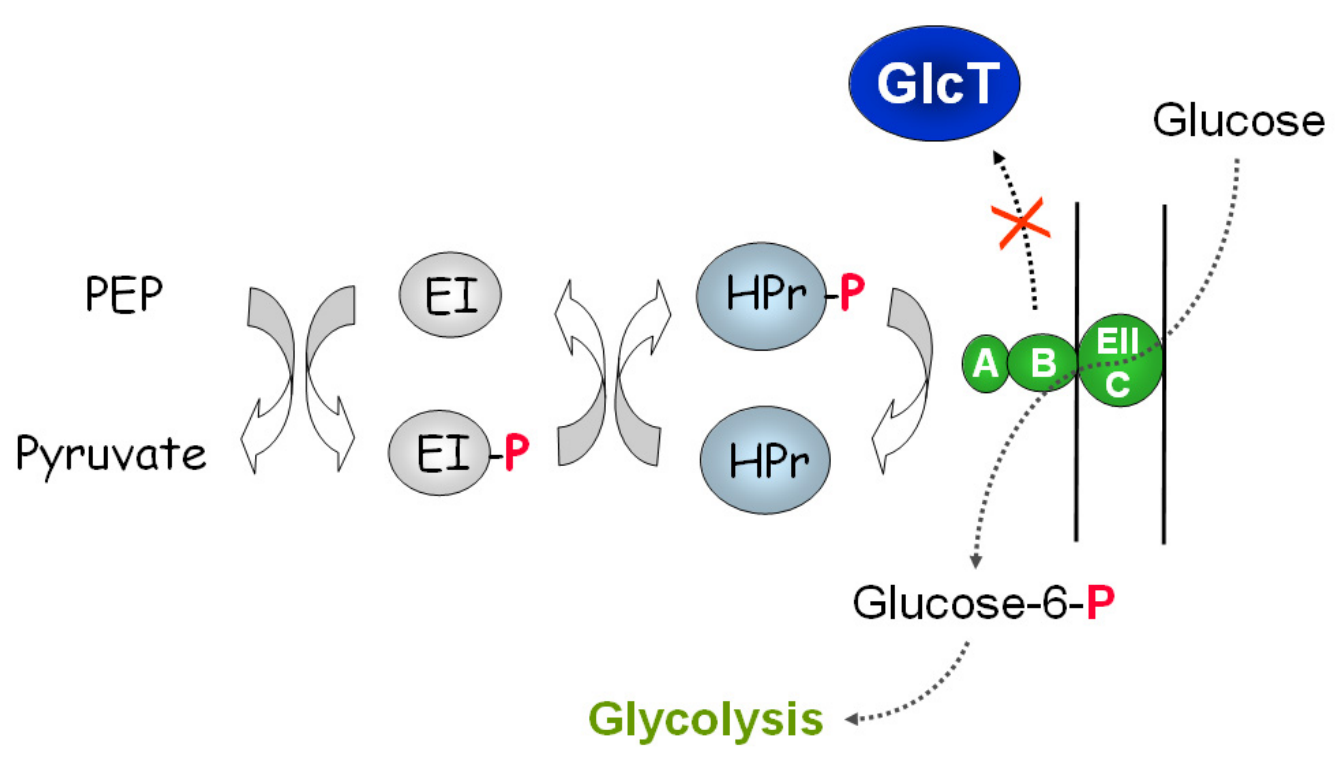

Figure 1.5. Schematic overview on the glucose-specific phosphotransferase system (PTS) of B. subtilis. The phosphoryl residue (-P) is transferred from PEP via enzyme I (EI), the HPr protein (His-15 residue), and enzymes EIIA (A) and EIIB (B) to the incoming glucose. The glucose uptake is mediated by the membrane spanning enzyme EIIC. If glucose is available, the phosphate residue is transferred to the incoming glucose. In the absence of glucose, the phosphate residue is transferred to the transcriptional antiterminator protein GlcT. Modified from Bachem and Stülke, 1998. 
In the presence of a PTS sugar the general components and the sugar specific permease are mainly in a non-phosphorylated state. If the sugar for a certain permease is not present, the phosphate accumulates at that permease. In such a case, the phosphate can be transferred to a regulatory protein.

The first of these proteins described was BglG from E. coli (Amster-Choder et al., 1989; Amster-Choder and Wright, 1992; Mahadevan and Wright, 1987). It modulates transcriptional antitermination of the $b g l$-operon and is therefore called "antiterminator protein". The activity of this "antiterminator" protein is controlled by the phosphorylation state of its cognate EII, i.e. BglF (Amster-Choder et al., 1989). This control mechanism involves a phosphoryl-transfer from EII to the antiterminator protein, leading to its inactivity in the absence of $\beta$-glucosides. In the presence of $\beta$-glucosides, the antiterminator protein remains in its unphosphorylated state and prevents transcription termination of its target RNA, the bglP-operon (Amster-Choder and Wright, 1993). In B. subtilis, there are four antiterminator proteins of the BglG family. These regulatory proteins can bind to RNA structures found in the 5'-UTR of some genes involved in the uptake and metabolism of the appropriate sugars. Upon binding, they prevent the formation of intrinsic terminators and enable transcription of the coding genes.

\subsubsection{Regulation of the ptsGHI operon by transcriptional antitermination}

The ptsGHI operon of B. subtilis codes for the sugar specific permease $\mathrm{EII}^{\mathrm{Glc}}(p t s G)$, and the general components $\operatorname{HPr}(p t s H)$ and Enzyme I ( $p t s I)$. Although there are constitutive promoters in front of ptsG and ptsHI, only HPr and EI are constitutively synthesized (GonzyTréboul et al., 1989; Stülke et al., 1997). High amounts of EII ${ }^{\mathrm{Glc}}$ can only be found if glucose is available. The pts $G$ mRNA is only found when glucose is present, which indicates that $p t s G$ might be controlled at the level of transcription. Further studies revealed that $p t s G$ expression is controlled by premature transcription termination (Stülke et al., 1997). If glucose is not available, only a short transcript of $p t s G$ is made. A terminator stem-loop located between the promoter region and the coding region of $p t s G$ is formed, causing transcriptional antitermination. In the presence of glucose, a second structure called RAT (RNA antiterminator) is formed, preventing terminator formation (see Fig. 1.6) (Langbein et al., 1999). Thus, the regulation mode of the $p t s G$ RNA switch is similar as described in Figure 1.4c. However, unlike metabolite binding riboswitches, the regulation of the pts $G$ RNA switch is not mediated by a direct glucose-RNA interaction. Instead, the regulatory 
protein GlcT causes transcriptional antitermination in the presence of glucose (Stülke et al., 1997).

The $g l c T$ gene is located upstream of the $p t s G H I$ operon, and its transcription is under the control of a constitutive promoter (see Fig. 1.6). The activity of GlcT is controlled by a phosphorylation event that indirectly correlates to the glucose concentration. When glucose is not present, GlcT is phosphorylated. Phosphorylated GlcT is inactive and does not bind the RAT RNA sequence. When glucose is present, GlcT remains in its unphosphorylated but active state (see Fig. 1.5).

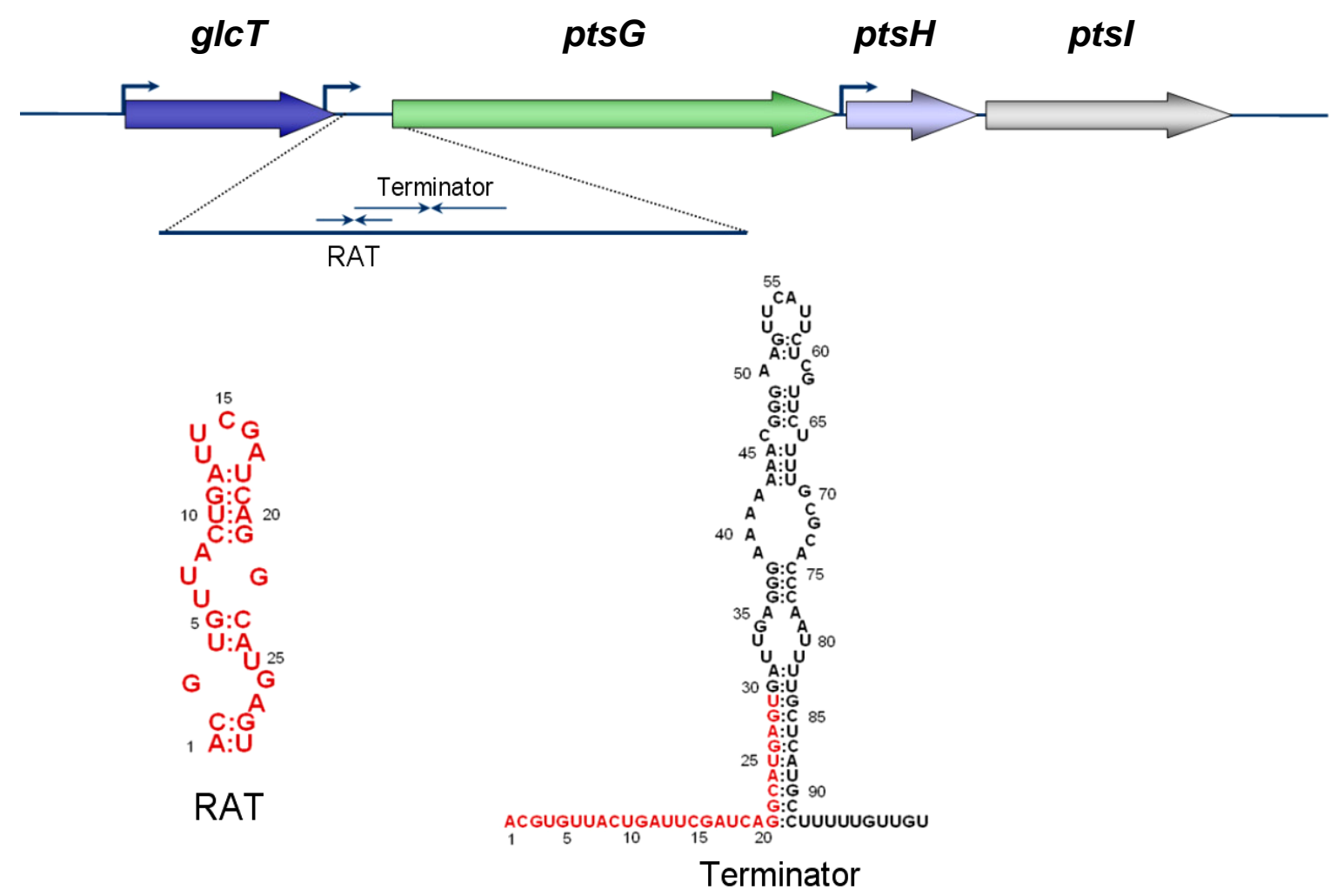

Figure 1.6. The ptsGHI operon and a proposed model of the antiterminator and terminator structures of the ptsG leader mRNA. The $g l c T$ gene is located upstream of the $p t s G H I$ operon. The $p t s G H I$ operon codes for the glucose specific permease $p t s G$, and the general PTS enzymes HPr and Enzyme I ( $p t s I)$. The promoters shown upstream of $g l c T$ and $p t s G H I$ as well as the promoter upstream of $p t s H$ are constitutively expressed.

In the untranslated region of $p t s G$, the RNA sequence can form two different secondary structures. The terminator is the thermodynamically more stable structure and causes transcriptional termination when formed. The formation of the RAT (RNA antiterminator) structure needs to be stabilized by the protein factor GlcT. Since RAT and terminator are overlapping sequences, both structures are mutually exclusive structures. Modified from Stülke and Hillen, 2000. 
GlcT consists of an RNA-binding domain (RBD), and two PTS regulatory domains (PRD-I and PRD-II, see Fig. 1.7) (Stülke et al., 1998). If no glucose is present, GlcT is phosphorylated at PRD-I by EII ${ }^{\mathrm{Glc}}$ and thereby inactivated (Schmalisch et al., 2003). However, if glucose is present, the sugar is phosphorylated rather than PRD-I by EII ${ }^{\mathrm{Glc}}$ and GlcT becomes active. Active GlcT binds as a dimer of two identical subunits to its RNA target sequence and stabilizes the RAT secondary structure. As the terminator sequence overlaps the RAT sequence partially, both structures are mutually exclusive (see Fig. 1.6). Thus, the formation of the RAT structure with the help of GlcT suppresses the thermodynamically more stable terminator and enables the transcription into the coding gene. It was also demonstrated that a protein consisting only of the RBD is sufficient to cause antitermination at the ptsGHI operon (Bachem and Stülke, 1998). This shortened protein can dimerize and binds to the RAT-sequence. As it can not be inactivated due to the lack of PRD-I, it shows constitutive activity and thus causes constitutive expression of $p t s G$. Besides the negative phosphorylation of GlcT by the sugar permease EII ${ }^{\mathrm{Glc}}$ at PRD-I, the antiterminator protein can also be phosphorylated at PRD-II by HPr. HPr dependent phosphorylation of active GlcT at PRD-II has a slightly positive effect on the GlcT activity (Schmalisch et al., 2003).

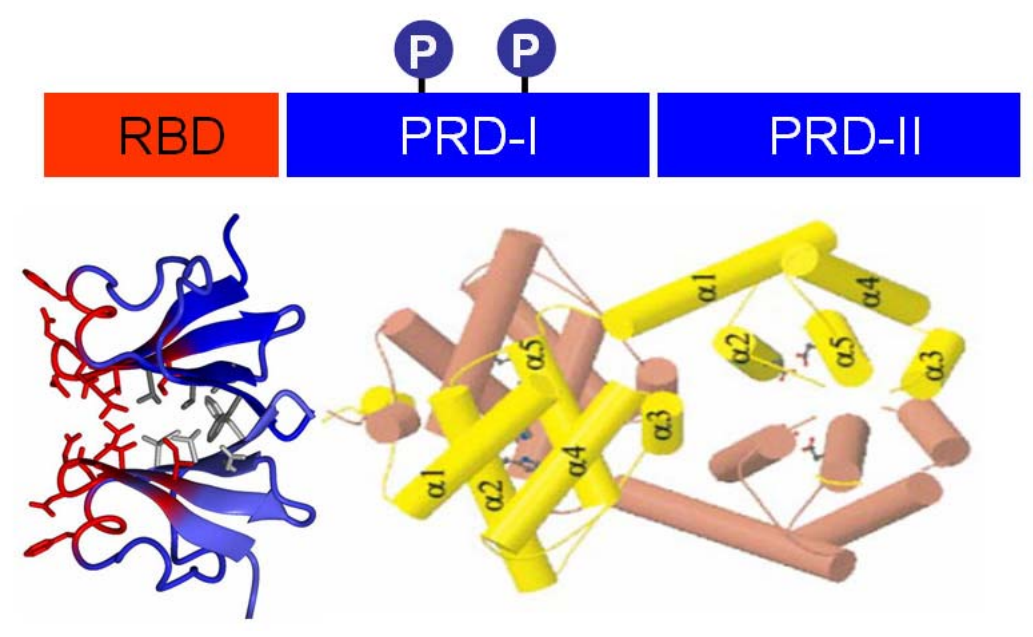

Figure 1.7. Schematic illustration of the antiterminator protein GlcT. Up to now, the structure of the full length GlcT protein could not be obtained. The picture of the RNA-binding domain (RBD) was taken from an NMR structure of the GlcT-RBD (Müller, 2001). PRD-I and PRD-II are taken from the crystal structure of LicT (van Tilbeurgh et al., 2001). The phosphorylation sites of PRD-II are not shown as they are not relevant for GlcT activity. 


\subsubsection{The family of homologous antiterminator systems of $B$. subtilis}

In B. subtilis, there are four PTS controlled antiterminator proteins regulating the transcription of several genes (see Fig. 1.8). These antiterminator proteins have all the same domain organization as GlcT (see Fig. 1.7) (Manival et al., 1997; Stülke et al., 1998; Tortosa et al., 1997). The PRD-I is negatively phosphorylated by the cognate EII if the appropriate sugar is not available, whereas the PRD-II is positively phosphorylated by HPr if no better carbon source is available. Similar than GlcT does for the ptsGHI operon, LicT controls the transcription of the $\operatorname{bglPH}$ operon and $\operatorname{licS}$ ( $\beta$-glucoside permease, phospho- $\beta$-glucoside hydrolase, and $\beta$-1,3-1,4-glucanase) by transcriptional antitermination. These genes are involved in the utilization of aryl- $\beta$-glucosides and $\beta$-glucans (Krüger and Hecker, 1995; Schnetz et al., 1996). The sugar permease BglP controls LicT activity by phosphorylation of the PRD-I, depending on the availability of $\beta$-glucosides (Lindner et al., 1999). The antiterminator protein SacT controls the $\operatorname{sacPA}$ operon (sucrose permease and sucrase). SacP mediates the uptake of sucrose, and SacT activity is controlled by SacP dependent phosphorylation of PRD-I (Arnaud et al., 1996; Arnaud et al., 1992).

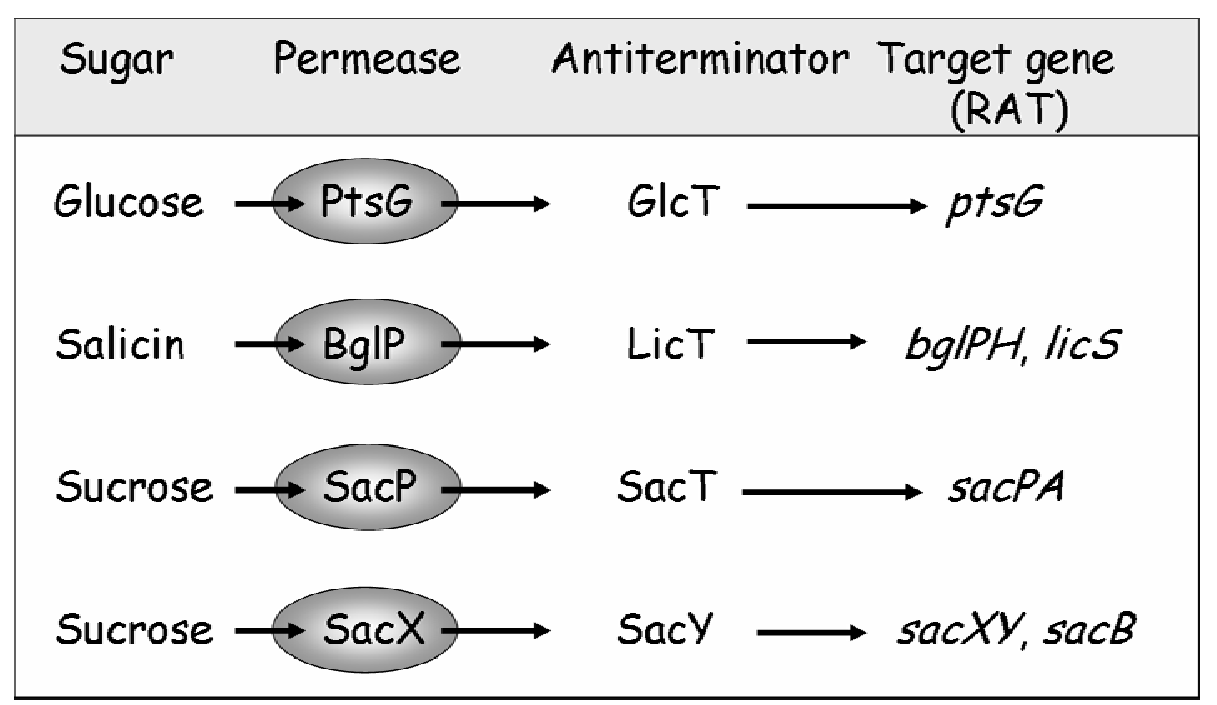

Figure 1.8. A family of antitermination systems controls sugar uptake and metabolism in B. subtilis. The design of the four signaling systems. The target genes encode the following proteins: $p t s G$, glucose permease of the PTS; $b g l P, \beta$-glucoside permease of the PTS; $b g l H$, phospho- $\beta$ glucoside hydrolase; licS, $\beta$-1,3-1,4-glucanase; sacP, sucrose permease of the PTS; sacA, sucrase; $s a c X$, sucrose permease of the PTS (low affinity); $s a c Y$, antitermination protein; $s a c B$, extracellular levansucrase. 
SacY activity is controlled by SacX, which codes for a low affinity sucrose permease. SacX only transports sucrose when the extracellular sucrose concentration is high. Thus, SacY is only active when plenty of sucrose is available. It controls the $\operatorname{sacXY}$ operon and $s a c B$ (extracellular levansucrase) (Arnaud et al., 1996; Crutz et al., 1990; Tortosa et al., 1997). To become active, LicT and SacT need to be dephosphorylated at PRD-I and positively phosphorylated at PRD-II (Arnaud et al., 1996; Lindner et al., 1999). When glucose is simultaneously available with sucrose or $\beta$-glucosides, the PRD-I of the relevant antiterminator protein is dephosphorylated, but the HPr dependent positive phosphorylation is lacking as most of the HPr molecules phosphorylate the E-II ${ }^{\mathrm{Glc}}$ and not the PRD-II of the respective antiterminator proteins. This prevents the activity of SacT or LicT in the presence of the preferred carbon source glucose. The PRD-II of GlcT and SacY can also be phosphorylated by HPr, but the activity of GlcT and SacY is not severely influenced by the phosphorylation state of PRD-II (Bachem and Stülke, 1998; Schmalisch et al., 2003; Tortosa et al., 1997).
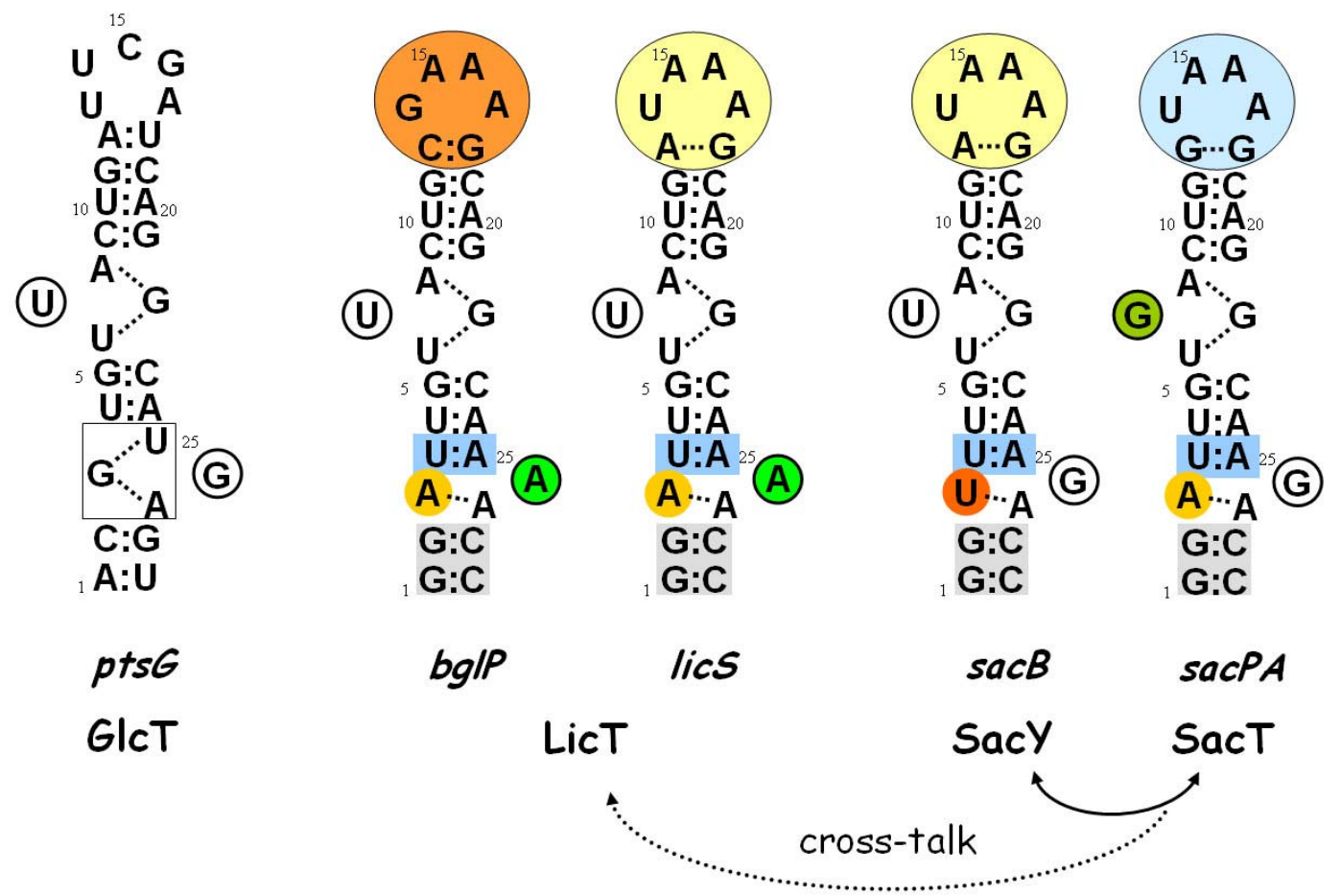

Figure 1.9. Proposed secondary structures for the related RAT RNAs in B. subtilis (Yang et al., 2002). The relevant antiterminator proteins are indicated below their cognate RAT structures. Boxes and circles indicate nucleotides that differ from the $p t s G$ RAT. Dashed lines indicate bases that are proposed to be in direct contact to each other. The main differences are located in the top loop and lower loop structures. The pts $G$ RAT holds an outstanding position due to its unique lower stem loop and the triple base pairing in the lower loop region. Binding of sucrose antiterminator proteins to RAT sequences of $b g l$ genes was reported previously. Also, cross-talk within the sucrose related antiterminator systems has been revealed (Aymerich and Steinmetz, 1992). 
Since all these proteins bind their target RNA as a dimer, it is most likely that the phosphorylation state of the PRDs modulates the dimerization ability of the two identical subunits. Although the four antiterminator proteins are part of four separate regulatory systems, the proteins are highly similar to each other. Not only the basic structures of the proteins are identical (see Fig. 1.7), but also the domains RBD, PRD-I, and PRD-II are each very similar among the related antiterminator proteins (Débarbouillé et al., 1990; Greenberg et al., 2002; Stülke et al., 1998; Tortosa et al., 1997). But not only the proteins share high homology, also the cognate RAT structures of the different systems share high similarity in sequence and structure (see Fig. 1.9) (Aymerich and Steinmetz, 1992; Yang et al., 2002). The antiterminator proteins of the sucrose systems, SacT and SacY, seem to be able to recognize the RAT structure of their respective counterpart. It was also reported that the antiterminator proteins of the sucrose systems can bind to the RATs of the $b g l$ genes (Aymerich and Steinmetz, 1992; Steinmetz et al., 1989). However, neither can LicT bind to $s a c B$ or $s a c P$ RATs, nor does sucrose induce licS or the $b g l P H$ operon. The GlcT/pts $G$ RAT couple is the most distant system and no cross-talk to other systems has been observed.

In general, not only the RAT/antiterminator protein recognition, but also the positive and negative phosphorylation of the PRDs, and also carbon catabolite repression, which limits the expression of some systems under certain conditions, contribute to the regulatory specificity. 


\subsection{Aim of this work}

B. subtilis is one of the best-studied organisms so far. Although much effort has been devoted to the elucidation of the genes involved in central metabolism, not much is known about their regulation and the regulatory networks by which each individual pathway is connected to the others. In this work, a global approach combining DNA microarrays and metabolic flux analysis should allow to draw conclusions not only about gene regulation on a transcriptomic level, but also about regulatory events on protein level. Therefore, the first aim was the design and establishment of working methods for global screening systems based on transcriptome and fluxome analysis. As a first proof of concept, the influence of organic acids, like glutamate and succinate, on the metabolism of B. subtilis in addition to glucose should be determined in minimal medium.

The PTS is part and also trigger of regulatory events in carbohydrate metabolism in B. subtilis. Some of the involved genes are regulated by premature transcription termination controlled by a protein dependent RNA switch. In B. subtilis, there is a family of four systems sharing the same mode of regulation. The components of these systems are highly conserved among each other and cross-talk for some systems was reported in vitro. The GlcT/ptsG system holds an outstanding position. Neither does GlcT bind any other riboswitch structures than that of ptsG RAT, nor do any other antiterminator proteins than GlcT bind to this riboswitch. In vivo only the genes needed for the utilization of the currently available carbon source are activated. Moreover, if several sugars are available, the bacterium only metabolizes the best carbon source. The second aim of this work was the characterization of the specificity determinants of the GlcT/ptsG antiterminator system and in a further approach the characterization of all factors needed to maintain specificity for the four different systems.

As site-directed mutagenesis was a major tool to determine the specificity determinants of the different protein/RAT couples, a fast and efficient method for the introduction of multiple mutations in the RAT sequences should be developed. 


\section{Transcriptional and metabolic responses of Bacillus subtilis to the availability of organic acids: Transcription regulation is important but not sufficient to account for metabolic adaptation}

The results described in this chapter were published in:

Schilling, O., O. Frick, C. Herzberg, A. Ehrenreich, E. Heinzle, C. Wittmann, and J.Stülke. 2007. Transcriptional and metabolic responses of Bacillus subtilis to the availability of organic acids: Transcription regulation is important but not sufficient to account for metabolic adaptation. Appl Environ Microbiol 73:499-507.

Author contributions:

This study was designed by O.S., O.F., C.W., E.H., and J.S.. O.S. performed and interpreted all reporter gene and transcriptome analyses. O.F. performed and interpreted all fluxome analyses. C.H. contributed substantially to all lab work related to transcriptome analysis. A.E. provided the equipment needed for microarray design and analysis. J.S. and O.S. wrote the paper. 


\begin{abstract}
The soil bacterium Bacillus subtilis can use sugars or organic acids as sources of carbon and energy. These nutrients are metabolized by glycolysis, the pentose phosphate pathway, and the Krebs citric acid cycle. While the response of $B$. subtilis to the availability of sugars is well understood, much less is known about the changes in metabolism if organic acids feeding into the Krebs cycle are provided. If B. subtilis is supplied with succinate and glutamate in addition to glucose, the cells readjust their metabolism as determined by transcriptome and metabolic flux analyses. The portion of glucose-6-phosphate that feeds into the pentose phosphate pathway is significantly increased in the presence of organic acids. Similarly, important changes were detected at the level of pyruvate and acetyl coenzyme A (acetyl-CoA). In the presence of organic acids, oxaloacetate formation is strongly reduced, whereas the formation of lactate is significantly increased. The als $S D$ operon required for acetoin formation is strongly induced in the presence of organic acids; however, no acetoin formation was observed. The recently discovered phosphorylation of acetolactate decarboxylase may provide an additional level of control of metabolism. In the presence of organic acids, both types of analyses suggest that acetyl-CoA was catabolized to acetate rather than used for feeding the Krebs cycle. Our results suggest that future work has to concentrate on the posttranslational mechanisms of metabolic regulation.
\end{abstract}

\title{
Introduction
}

Bacillus subtilis and its close relatives are among the most important industrial microorganisms. They are used for the production of antibiotics, enzymes such as proteases, amylases, lipases, or cellulases, vitamins such as riboflavin, and even insecticidal proteins. Due to this practical relevance, much research has been devoted to many aspects of these bacteria, making B. subtilis one of the best-studied living organisms.

The basis for understanding the potential of $B$. subtilis is a firm knowledge of its metabolism. These bacteria prefer sugars and ammonia as carbon and nitrogen sources, respectively. The preferred source of carbon and energy is glucose. This sugar is taken up and concomitantly phosphorylated by the bacterial phosphoenolpyruvate:sugar phosphotransferase system. The further metabolism involves glycolysis, the pentose phosphate pathway, and the Krebs cycle, resulting in the oxidation of glucose to carbon 
dioxide and the generation of ATP, a proton-motive force, reducing power, and intermediates for all anabolic reactions. Similarly, other sugars and polyols can be phosphorylated and are catabolized in a similar manner (for a review see Stülke and Hillen, 2000). The utilization of organic acids requires the conversion of these acids to intermediates of the Krebs cycle, gluconeogenesis, and the pentose phosphate pathway to obtain both the energy and the metabolic precursors needed by the cell (Doan et al., 2003). The central intermediate of nitrogen metabolism is glutamate, the universal amino-group donor for the biosynthesis of amino acids, nucleotides, and all other nitrogen-containing compounds in the cells (for a review see Fisher, 1999).

The details of the central carbon and nitrogen metabolic pathways in B. subtilis and their regulation have been the subject of extensive investigations. The ptsGHI operon, encoding the general and glucose-specific phosphotransferase system proteins, is induced by glucose due to a mechanism of transcriptional antitermination (Schmalisch et al., 2003; Stülke et al., 1997). The glycolytic enzymes that are needed for reversible reactions are constitutively transcribed, whereas those that catalyze irreversible reactions ( $p f k A, g a p A$, and pyk, encoding phosphofructokinase, glyceraldehyde-3-phosphate dehydrogenase, and pyruvate kinase, respectively) are induced in the presence of glucose (Fillinger et al., 2000; Ludwig et al., 2001). The complex gapA operon, containing six genes encoding enzymes of triose phosphate interconversions, is repressed by the CggR repressor in the absence of glycolytic substrates, and the mRNA is in addition subject to posttranscriptional processing. Moreover, an internal promoter ensures that those enzymes catalyzing reversible reactions are present under both glycolytic and gluconeogenic conditions (Doan and Aymerich, 2003; Meinken et al., 2003). The pdhABCD operon, encoding pyruvate dehydrogenase, the link between glycolysis and the Krebs cycle, is also induced by glucose by an as-yet-unknown mechanism (Blencke et al., 2003). Several genes encoding enzymes of the Krebs cycle, such as $c i t Z$ and $c i t B$, coding for the major citrate synthase and aconitase, respectively, are subject to a synergistic repression by glucose and a preferred nitrogen source such as glutamine. This repression is mediated by the LysR-type repressor protein CcpC (Blencke et al., 2006; Jourlin-Castelli et al., 2000). Even though it is important under all conditions, not much is known about the regulation of the pentose phosphate pathway. A recent transcriptome study suggested that the genes encoding the enzymes of this pathway are constitutively expressed (Blencke et al., 2003).

The assimilation of ammonium is initiated by its uptake by diffusion and by the AmtB (also designated $\mathrm{NrgA}$ ) ammonium transporter at high and low ammonium concentrations, 
respectively (Detsch and Stülke, 2003). In B. subtilis, ammonium is assimilated exclusively by the glutamine synthetase/glutamate synthase pathway. Briefly, glutamine is synthesized from glutamate and ammonium, and the glutamine is used as the amino-group donor for the production of two molecules of glutamate with 2-oxoglutarate as the acceptor (Belitsky et al., 2000). The gene encoding glutamine synthetase $(g \ln A)$ is controlled by the availability of glutamine through the GlnR repressor (Schreier et al., 1989). The gltAB operon encoding glutamate synthase is subject to a dual control by the carbon and nitrogen sources: in the absence of ammonium, expression of the operon is repressed by the TnrA transcription factor, whereas transcription is activated by the GltC protein in the presence of sugars (Belitsky et al., 2000; Commichau et al., 2006; Wacker et al., 2003).

Table 1. B. subtilis strains used in this study

\begin{tabular}{|c|c|c|}
\hline Strain & Genotype & Source $^{\mathrm{a}}$ \\
\hline 168 & $\operatorname{trp} C 2$ & Laboratory collection \\
\hline BSIP1114 & $\operatorname{trpC} 2$ amyE::(pta-lacZ cat) & Presecan-Siedel et al., 1999 \\
\hline GP133 & $\operatorname{trpC} 2$ amyE::(fbaA-lacZ cat $)$ & Ludwig et al., 2001 \\
\hline GP205 & $\operatorname{trp} C 2$ amyE::(citB-lacZ cat $)$ & Blencke et al., 2006 \\
\hline GP216 & $\operatorname{trpC} 2$ amyE::(pdhA-lacZ cat $)$ & $\mathrm{pGP} 721 \rightarrow 168^{\mathrm{a}}$ \\
\hline GP250 & $\operatorname{trp} C 2$ amyE::(nrgA-lacZ aphA3) & Detsch and Stülke, 2003 \\
\hline GP268 & $\operatorname{trpC} 2$ amyE::(glnR-lacZ aphA3) & $\mathrm{pGP} 189 \rightarrow 168^{\mathrm{a}}$ \\
\hline GP313 & $\operatorname{trpC} 2$ amyE::(cggR-lacZ cat $)$ & Ludwig et al., 2001 \\
\hline GP314 & $\operatorname{trpC} 2$ amyE::(pgi-lacZ cat $)$ & Ludwig et al., 2001 \\
\hline GP315 & $\operatorname{trpC2}$ amyE::(pfkA-lacZ cat) & Ludwig et al., 2001 \\
\hline GP317 & $\operatorname{trp} C 2$ amyE::(pgk-lacZ cat $)$ & Ludwig et al., 2001 \\
\hline GP342 & $\operatorname{trpC} 2$ amyE::(gltA-lacZ aphA3) & Wacker et al., 2003 \\
\hline QB5556 & $\operatorname{trpC2}$ amyE::(rocD'-'lacZ cat) & Gardan et al., 1995 \\
\hline QB7041 & $\operatorname{trpC} 2$ amyE::( $\Delta \mathrm{CA} p t s G-l a c Z$ cat $)$ & Stülke et al., 1997 \\
\hline
\end{tabular}

${ }^{a}$ Arrows indicate construction by transformation.

While much work has been devoted to the effects of the presence of glucose on gene expression and the cellular physiology of B. subtilis, relatively little is known about the response towards organic acids that feed into the Krebs cycle. These intermediates of the Krebs cycle and glutamate are known to induce specific transporters (Asai et al., 2000; Tanaka et al., 2003; Yamamoto et al., 2000). Moreover, glutamate represses the genes of the Krebs cycle as long as ammonium and glucose are also available (Blencke et al., 2006; 
Sonenshein, 2002). The impact of these Krebs cycle intermediates on the general metabolism has not been studied so far.

In this work, we investigated the response of B. subtilis to the presence of organic acids. This response was determined at the level of the subtranscriptome of genes of the central carbon and nitrogen metabolism and by assaying the carbon fluxes. Our results indicate that overflow metabolism, the Krebs cycle, and the reactions involved in the interconversions between glutamate and 2-oxoglutarate are strongly affected by the presence of the organic acids. Importantly, the data allow us to derive conclusions relating to possible posttranscriptional regulation events that had escaped attention until now.

\section{Materials and Methods}

\section{Bacterial strains, growth conditions, and enzyme assays}

The $B$. subtilis strains used in this study are listed in Table 1 . They are all isogenic derivatives of the strain 168. Bacteria were grown in $\mathrm{C}$ minimal medium supplemented with the auxotrophic requirement (tryptophan at $50 \mathrm{mg}^{-1}$ ) (Faires et al., 1999). Other media consisted of $\mathrm{C}$ medium supplemented with $99 \%\left[1-{ }^{13} \mathrm{C}\right]$ glucose (CG medium) or $\mathrm{C}$ medium supplemented with a mixture of $99 \%\left[1-{ }^{13} \mathrm{C}\right]$ glucose, succinate, and glutamate (CGSE medium). The labeled glucose was obtained from Campro Scientific (Veenendaal, The Netherlands). Glucose, succinate, and glutamate were added to final concentrations of $0.5 \%$, $0.6 \%$, and $0.8 \%$, respectively (Faires et al., 1999). Parallel cultivations were performed in $500-\mathrm{ml}$ shake flasks using a culture volume of $50 \mathrm{ml}$. The resulting physiological data on CG and CGSE are listed below (Table 2). Aliquots from identical cultures grown to mid-log phase (optical density at $600 \mathrm{~nm}$ of 1.5 ) were used for transcriptome and metabolic flux analyses. Quantitative assays of lacZ expression in B. subtilis were performed with cell extracts using $o$-nitrophenyl- $\beta$-galactopyranoside as the substrate, as described previously (Martin-Verstraete et al., 1992).

\section{Plasmid constructions}

Translational fusions with the lac $Z$ gene were constructed using the vector pAC5 (MartinVerstraete et al., 1992), which carries the pC194 chloramphenicol resistance gene cat, or the vector pAC7 (Weinrauch et al., 1991), containing the kanamycin resistance gene aphA3. 
Table 2. Specific rates of growth, substrate consumption, and product formation and yields of B. subtilis cultivated on CG and CGSE

\begin{tabular}{lcc}
\hline & \multicolumn{2}{c}{ Result for cultivation on: } \\
\cline { 2 - 3 } Measurement & CG & CGSE \\
\hline Specific growth rate $\mu\left[\mathrm{h}^{-1}\right]$ & 0.64 & 0.81 \\
Specific rates $\left[\mathrm{mmol} \mathrm{g}^{-1} \mathrm{~h}^{-1}\right]$ for: & & \\
Glucose & -9.1 & -10.0 \\
Succinate & 0 & -1.0 \\
Glutamate & 0 & -4.2 \\
Acetate & 5.3 & 8.3 \\
Lactate & 0 & 2.5 \\
Pyruvate & 0.1 & 0.6 \\
& & \\
Yield coefficients $\left[\mathrm{mol} \mathrm{mol}^{-1}\right]$ for: & & 0.08 \\
Acetate & 0.58 & 0.83 \\
Lactate & 0 & 0.06 \\
Pyruvate & 0.02 & 0.06 \\
2-Oxoglutarate & 0.01 & \\
Cell dry mass $\left[\mathrm{g} \mathrm{mmol}^{-1}\right]$ & 0.07 & \\
\hline
\end{tabular}

Both plasmids harbor a lacZ gene without a promoter located between two fragments of the B. subtilis amyE gene. Plasmid pGP189, containing a glnR-lacZ fusion, was constructed as follows. A 331-bp fragment containing the promoter region of the $g \ln R A$ operon was amplified by PCR using the primers CD43 (5' AAAGAATTCCATTATGGCA GCAGGGACGTT) and CD44 (5' TCCTATTGGATCCAAAGGCATTGAGCG). The PCR products were digested with EcoRI and BamHI (restriction sites were introduced by PCR and are underlined in the primer sequences) and ligated with pAC7 linearized with the same enzymes. Similarly, plasmid pGP721, used for the construction of the $p d h A$-lacZ fusion, was obtained using the primers HMB49 (5' CGGAATTCGATGCTGCAGGCTATCGTG) and HMB50 (5' CGGGATCCGCTTTTTTCGTTTTTGCAGCC) with pAC5 as the cloning vector. 


\section{RNA isolation and quality assessment}

To isolate high-quality RNA suited for the detection of long transcripts and their precursors, the following procedure was used. The cells were harvested at the exponential phase. For RNA preparation, $12 \mathrm{ml}$ of a cell suspension (optical density at $600 \mathrm{~nm}$ of 1.5 ) was used. After mechanical cell disruption, the frozen powder was instantly resuspended in $3 \mathrm{ml}$ lysis buffer (4 M guanidine isothiocyanate, $0.025 \mathrm{M}$ sodium acetate, $\mathrm{pH} 5.3,0.5 \%$ [wt/vol] $N$-laurylsarcosine). Subsequently, total RNA extraction with acid phenol solution was performed as described previously (Ludwig et al., 2001). The quality of the isolated RNA was tested by PCR to exclude the presence of traces of DNA and by Northern blot analysis to detect a large nonabundant mRNA (7.4 kb mRNA of the gapA operon). Digoxigenin RNA probes specific for gapA (Tobisch et al., 1999b) were used to detect the transcript of the gapA operon. The sizes of the RNA molecular weight markers (GIBCO BRL) were as follows: $9.49,7.46,4.40,2.37,1.35$, and $0.24 \mathrm{~kb}$.

\section{Transcriptome analysis}

The microarray slides contained 93 different PCR products of 300 to $500 \mathrm{bp}$ in length, representing 70 genes of the central carbon and nitrogen metabolic pathways of B. subtilis. The PCR products correspond to the 3 ' end of each gene. Additionally, selected genes were also present on the slides with 300 to $500 \mathrm{bp}$ of the $5^{\prime}$ ends as a quality control. To avoid PCR artifacts due to unspecific priming, all PCR products were checked on an agarose gel and sequenced. The PCR products were purified using a QIAquick 96 PCR purification kit (QIAGEN, Hilden, Germany) on a BioRobot 8000 (QIAGEN, Hilden, Germany). The slides were produced by spotting the PCR products using a Lucidea (Amersham Biosciences, Freiburg, Germany) contact printer. Each gene was present with eight spots organized in two rows with four spots on the microarray slide. Each row originated from an independent PCR. For laser calibration, a custom-made scorecard, consisting of 10 PCR products of Escherichia coli and Mycoplasma pneumoniae genes (400 to $500 \mathrm{bp}$ each), was used. These DNA fragments were spotted, and the absence of cross-hybridization with any of the labeled B. subtilis cDNAs was verified. Each spot contained $30 \mathrm{pg}$ of probe DNA and was generated by placing $100 \mathrm{pl}$ of a $50 \%$ aqueous dimethyl sulfoxide solution on amino-silane-coated Type 7* slides (Amersham Biosciences, Freiburg, Germany). The probes were cross-linked onto the slides by UV illumination $\left(50 \mathrm{~mJ} / \mathrm{cm}^{2}\right)$ after the total evaporation of the spotting solution. The arrays were stored in the dark in a vacuum desiccator. 


\section{cDNA labeling}

The isolated RNA was used to generate cDNAs labeled with fluorescent Cy-3 and Cy-5 dyes according to a direct labeling protocol (Hovey et al., 2005) with the following modifications. Annealing was performed with $25 \mu \mathrm{g}$ of total B. subtilis RNA, $40 \mathrm{mU}$ of RNA Guard (Amersham Biosciences, Germany), in vitro transcribed RNA of the 10 E. coli and M. pneumoniae genes (ranging from 1.5 to $25 \mathrm{ng}$ ) with defined ratios for the $\mathrm{Cy}-3$ and $\mathrm{Cy}-5$ reaction mixes $(1: 10,10: 1,1: 5,5: 1$, and $1: 1)$, and $1 \mu 1$ specific primer mix (including primers of the 10 scorecard genes). Each primer was present in the primer mix at a concentration of $5 \mathrm{pM}$. Primer annealing was performed in a thermocycler by asymptotic cooling from $70{ }^{\circ} \mathrm{C}$ to $22{ }^{\circ} \mathrm{C}$ within $30 \mathrm{~min}$. Then, fivefold reaction buffer, $200 \mathrm{U}$ Superscript III reverse transcriptase (Invitrogen GmbH, Karlsruhe, Germany), $2 \mu 1$ dithiothreitol (0.1 M), $1 \mu 1$ deoxynucleoside triphosphate mix (10 mM ATP, GTP, TTP, $3 \mathrm{mM} \mathrm{CTP}$ ), and $1 \mu \mathrm{Cy}-3$ or Cy-5 dyes (Amersham Biosciences, Germany) were added. Incubation was performed for 2 hours at $42{ }^{\circ} \mathrm{C}$. The labeled cDNA was purified with a CyScribe GFX purification kit (Amersham Biosciences, Germany). Finally, the amount of incorporated fluorescent nucleotides was determined by measuring the absorption of the purified labeled cDNA at 550 $\mathrm{nm}$ for Cy-3 and at $650 \mathrm{~nm}$ for Cy-5. In each hybridization, equal amounts of incorporated fluorescent dye, ranging from 80 to 150 pmol, were used.

\section{Hybridization}

For hybridization, the fluorescently labeled cDNA was denatured for $2 \mathrm{~min}$ at $95{ }^{\circ} \mathrm{C}$ followed by the addition of $50 \mu 1$ microarray hybridization buffer (CyScribe first-strand cDNA labeling kit; Amersham Biosciences, Germany) and $110 \mu$ formamide. Hybridization was carried out at $42{ }^{\circ} \mathrm{C}$ overnight using an automatic sample processor (Lucidea SlidePro hybridization chamber; Amersham Biosciences, Germany) (Hovey et al., 2005).

\section{Microarray analysis: data normalization and evaluation}

The signal intensities from each spot on the array were collected using a GenePix 4000B scanner and GenPix Pro software version 4.0 from Axon Instruments (Union City, CA). During a prescan at lower resolution, approximate normalization using the known concentrations of the scorecard was performed. The scan for data acquisition was then done at a $10-\mu \mathrm{m} /$ pixel resolution. The calculation of the expression ratios for all genes was performed using the GenPix Pro 4.0 software. Three mathematically different ratios were calculated: ratio of medians, ratio of means, and regression ratio. To ensure data quality, the following 
two criteria were applied: (i) the signal intensities of both fluorescent dyes of a spot corrected for its calculated background had to be greater than the standard deviation of the background of the corresponding fluorescent dye, and (ii) the deviation of the expression ratio calculated by the three different methods had to be within a $30 \%$ interval. To generate two biologically independent experiments, RNA was extracted from two independent cultures grown in CG or CGSE minimal medium. One pair of RNA samples was additionally subjected to a dye-flip experiment. As each open reading frame was spotted eight times on each microarray slide (see above), 24 expression values were generated for evaluation if each passed the quality control criteria. From at least 12 of the 24 potential values for each open reading frame, the average value of the ratio of median was calculated, and the standard deviations are shown elsewhere (see the supplemental material).

\section{Mass spectrometric ${ }^{13} \mathrm{C}$ labeling analysis}

Mass isotopomer fractions of amino acids from the cell protein were determined by gas chromatography-mass spectrometry (Kiefer et al., 2004; Wittmann et al., 2004). For this purpose, cells (about $1 \mathrm{mg}$ dry cell mass) were harvested from the culture and washed twice with deionized water. The pellet was then incubated with $50 \mu 16 \mathrm{M} \mathrm{HCl}$ for $24 \mathrm{~h}$ at $105^{\circ} \mathrm{C}$, subsequently neutralized with $6 \mathrm{M} \mathrm{NaOH}$, and cleared from insoluble matter by centrifugation (5 min; Ultrafree-MC filter units and 0.22- $\mu \mathrm{m}$ Durapore membrane; Millipore, Bedford, MA). The remaining clear solution was lyophilized. Analysis of the amino acids was performed after derivatization into the $t$-butyl-dimethylsilyl derivative (Krömer et al., 2004; Wittmann et al., 2002). All samples were first measured in scan mode to check for potential isobaric interference between analytes and other sample components. The labeling patterns of the amino acids were then determined in triplicate via selective ion monitoring of selected ion clusters, representing [M-57] fragments with the complete carbon skeletons of the amino acids. The mean experimental error for the mass isotopomer fractions was about $0.15 \%$.

\section{Metabolic modeling and parameter estimation}

The metabolic network for growth of B. subtilis on CG or CGSE comprised all central metabolic pathways, i.e. glycolysis, the pentose phosphate pathway, the Krebs cycle, and anaplerotic carboxylation. Additionally, the pathways for the formation of lactic acid, acetic acid, acetoine, and glycerol and the anabolic pathways from intermediary precursors into biomass were implemented. For glycine synthesis, two possible routes, i.e. via serine and via threonine aldolase, were considered (Simic et al., 2002). 
Table 3. Calculated and experimentally detected ${ }^{13} \mathrm{C}$ labeling patterns of amino acids in cell hydrolysates of $B$. subtilis cultivated on CG and CGSE

\begin{tabular}{|c|c|c|c|c|c|c|}
\hline \multirow{3}{*}{ Measured Metabolite ${ }^{a}$} & \multicolumn{6}{|c|}{ Result for cultivation on: } \\
\hline & \multicolumn{3}{|c|}{$\mathrm{CG}$} & \multicolumn{3}{|c|}{ CGSE } \\
\hline & $\mathrm{M}_{0}$ & $\mathrm{M}_{1}$ & $\mathrm{M}_{2}$ & $\mathrm{M}_{0}$ & $\mathrm{M}_{1}$ & $\mathrm{M}_{2}$ \\
\hline \multicolumn{7}{|l|}{ Alanine $\left(m / z 260, \mathrm{C}_{1-3}\right)$} \\
\hline calc. & 0.463 & 0.394 & 0.110 & 0.516 & 0.355 & 0.100 \\
\hline exp. & 0.462 & 0.398 & 0.110 & 0.517 & 0.357 & 0.098 \\
\hline \multicolumn{7}{|l|}{ Valine $\left(m / z 288, \mathrm{C}_{1-5}\right)$} \\
\hline calc. & 0.280 & 0.416 & 0.212 & 0.345 & 0.404 & 0.183 \\
\hline exp. & 0.279 & 0.419 & 0.212 & 0.344 & 0.405 & 0.183 \\
\hline \multicolumn{7}{|l|}{ Threonine $\left(m / z 404, \mathrm{C}_{1-4}\right)$} \\
\hline calc. & 0.305 & 0.388 & 0.206 & 0.560 & 0.273 & 0.123 \\
\hline exp. & 0.305 & 0.385 & 0.206 & 0.560 & 0.273 & 0.122 \\
\hline \multicolumn{7}{|l|}{ Aspartate $\left(m / z 418, \mathrm{C}_{1-4}\right)$} \\
\hline calc. & 0.305 & 0.388 & 0.206 & 0.559 & 0.273 & 0.124 \\
\hline exp. & 0.303 & 0.391 & 0.205 & 0.556 & 0.275 & 0.124 \\
\hline \multicolumn{7}{|l|}{ Glutamate $\left(m / z 432, \mathrm{C}_{1-5}\right)$} \\
\hline calc. & 0.205 & 0.365 & 0.264 & 0.629 & 0.231 & 0.107 \\
\hline exp. & 0.204 & 0.364 & 0.264 & 0.629 & 0.231 & 0.107 \\
\hline \multicolumn{7}{|l|}{ Lysine $\left(m / z 431, \mathrm{C}_{1-6}\right)$} \\
\hline calc. & 0.192 & 0.357 & 0.271 & 0.376 & 0.366 & 0.173 \\
\hline exp. & 0.198 & 0.352 & 0.267 & 0.374 & 0.367 & 0.174 \\
\hline \multicolumn{7}{|l|}{ Phenylalanine $\left(m / z 336, C_{1-9}\right)$} \\
\hline calc. & 0.2396 & 0.3946 & 0.2476 & 0.2478 & 0.3847 & 0.2449 \\
\hline exp. & 0.2389 & 0.3942 & 0.2477 & 0.2424 & 0.3943 & 0.2433 \\
\hline \multicolumn{7}{|l|}{ Tyrosine $\left(m / z 466, \mathrm{C}_{1-9}\right)$} \\
\hline calc. & 0.2063 & 0.3641 & 0.2616 & 0.2134 & 0.3564 & 0.2586 \\
\hline exp. & 0.2071 & 0.3645 & 0.2621 & 0.2123 & 0.3630 & 0.2558 \\
\hline
\end{tabular}

${ }^{a}$ calc., calculated values predicted by the solution of the mathematical model corresponding to the optimized set of fluxes; exp., experimental values obtained by gas chromatography-mass spectrometry analysis of $t$-butyl-dimethylsilyl-derivatized amino acids.

Calculation of the anabolic demands for the different precursors was based on literature data on the biomass composition of $B$. subtilis, previously determined as a function of growth rate (Dauner and Sauer, 2001; Dauner et al., 2002). The exact demands for both studied conditions were interpolated from these literature data depending on the specific growth rate measured in the present work (Table 2). 
Labeling data for proteinogenic amino acids and for glucose from all carbohydrates and the mean values of the stoichiometric data from two parallel cultivations were combined for a calculation of metabolic flux. The set of fluxes that gave minimum deviation between experimental $\left(\mathrm{M}_{\mathrm{i}, \exp }\right)$ and simulated $\left(\mathrm{M}_{\mathrm{i}, \text { calc }}\right)$ mass isotopomer fractions was taken as the best estimate for the intracellular flux distribution. The network was overdetermined, so that a least-squares approach was possible. As the error criterion, a weighted sum of least squares was used (Wittmann and Heinzle, 2002). All metabolic simulations were carried out on a personal computer by use of Matlab 7.0 (Mathworks Inc., Natick, MA). Details on the computational tools are given elsewhere (Wittmann and Heinzle, 2001; Wittmann and Heinzle, 2002; Wittmann et al., 2004). For the flux distributions, an excellent agreement between experimentally detected and calculated labeling patterns was achieved. The deviation between measured and calculated mass isotopomers was typically below 3\% and thus rather small (Table 3). Flux distributions, including deviations within intervals of $90 \%$ confidence, are shown in Fig. 2.1. The confidence intervals were calculated by a Monte Carlo approach as described previously (Wittmann and Heinzle, 2002).

\section{Results and Discussion}

\section{Regulation of glycolysis}

The expression of glycolytic genes was assayed by microarray analysis. The promoter activities of several glycolytic genes and operons were additionally studied by measuring $\beta$ galactosidase activities driven by these promoters. The transcriptome data suggested that the expression of glycolytic enzymes was only marginally affected by the addition of succinate and glutamate to the medium. These results are well supported by the comparison of the expression of lacZ fusions to promoters of glycolytic genes (Table 4). As judged from these expression data, the ptsGHI and gapA operons are highly transcribed under both conditions studied here, whereas the other promoters (pgi, pfkA, fbaA, and $p g k$ ) exhibit an intermediate activity (between 50 and 100 units of $\beta$-galactosidase/mg of protein). 


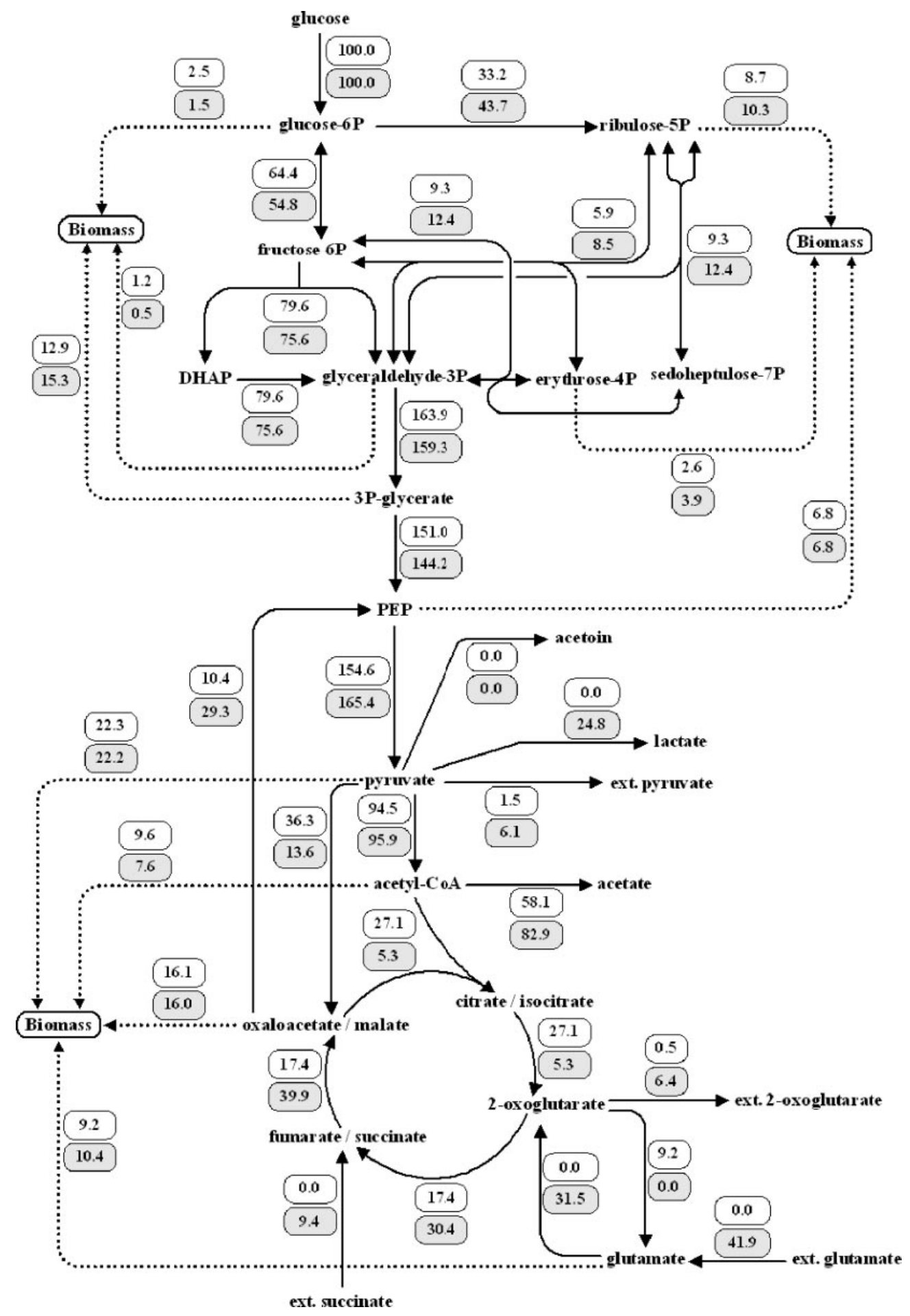

Figure 2.1. Intracellular carbon flux distribution of $B$. subtilis cultivated on $99 \%\left[1-{ }^{13} \mathrm{C}\right] \mathrm{glucose}$ under aerobic conditions in CG medium (white squares) and CGSE medium (gray squares). All fluxes are given as relative fluxes normalized to the specific glucose uptake rate (Table 2) including deviation values for $90 \%$ confidence. The fluxes correspond to the optimal fit between experimentally determined ${ }^{13} \mathrm{C}$ labeling patterns of amino acids of the cell protein and of glucose from cell carbohydrates and ${ }^{13} \mathrm{C}$ labeling patterns simulated via isotopomer modeling. PEP, phosphoenolpyruvate; DHAP, dihydroxyacetone phosphate; ext., external. 
Table 4. Expression of genes of central metabolism in B. subtilis as judged from transcriptional fusions to the $l a c Z$ gene ${ }^{\mathrm{a}}$

\begin{tabular}{cccccc}
\hline & & \multicolumn{2}{c}{$\beta$-Galactosidase activity } & \multicolumn{2}{c}{ CGSE/CG ratio by: } \\
\cline { 3 - 6 } Strain & Gene/ Operon & CG & CGSE & lacZ assay & $\begin{array}{c}\text { transcriptome } \\
\text { analysis }\end{array}$ \\
\hline QB7041 & $p t s G$ & 603 & 649 & 1.07 & 0.99 \\
GP314 & $p g i$ & 56 & 53 & 0.94 & 1.06 \\
GP315 & $p f k A$ & 63 & 93 & 1.47 & 1.30 \\
GP133 & $f b a A$ & 95 & 83 & 0.88 & 1.20 \\
GP313 & $c g g R(g a p A)$ & 393 & 496 & 1.26 & 1.13 \\
GP317 & $p g k$ & 35 & 55 & 1.56 & 1.25 \\
GP216 & $p d h A$ & 110 & 145 & 1.32 & 1.69 \\
BSIP1114 & $p t a$ & 50 & 220 & 4.30 & 1.67 \\
GP205 & $c i t B$ & 318 & 29 & 0.11 & 0.17 \\
GP250 & nrgA & 2 & 1.8 & 0.90 & 0.98 \\
GP268 & glnRA & 282 & 195 & 0.69 & 0.67 \\
GP342 & gltA & 295 & 172 & 0.58 & 0.42 \\
QB5556 & rocD & 12 & 12 & 1.00 & 1.17 \\
\hline
\end{tabular}

${ }^{a}$ The regulation of the indicated genes was determined using the results of the reporter gene studies as well as those of the transcriptome analysis.

${ }^{\mathrm{b}}$ Bacteria were grown in CG or CGSE minimal medium containing glucose. All strains contain transcriptional fusions of the promoter regions of the indicated genes to a promoterless lacZ gene. $\beta$-Galactosidase activities are given as units/mg of protein. Experiments were done in duplicate or triplicate. Representative results are shown.

These results are in good agreement with previous findings, which demonstrated induction of ptsGHI, gapA, and pfkA-pyk expression in the presence of glucose to levels similar to those observed in this study (Blencke et al., 2003; Ludwig et al., 2001).

The results of the transcription analysis demonstrate that the transcription of glycolytic genes and operons is unaffected by the presence of glutamate and succinate as long as glucose is present in the medium. This conclusion is supported and extended by the metabolic flux analysis: with the notable exception of the reaction catalyzed by phosphoglucoisomerase (Pgi), the flux of the carbon skeletons derived from glucose through glycolysis is not affected by the presence of the organic acids. Thus, the activity of the glycolytic enzymes remains constant in addition to their expression (with the exception of phosphoglucoisomerase [see below]). 


\section{The partition of glucose-6-phosphate and the pentose phosphate pathway}

Starting with glucose as the external substrate, glucose-6-phosphate is the first intracellular metabolite that is used by more than one enzyme. In CG minimal medium, glucose-6phosphate is used by the glycolytic enzyme phosphoglucoisomerase (Pgi), and about onethird is catabolized to ribulose-5-phosphate due to the activity of glucose-6-phosphate dehydrogenase (Zwf) and the further enzymes of the pentose phosphate pathway. Finally, a small amount (about 2.5\%) is used for the synthesis of biomass such as the cell wall. In the presence of glutamate and succinate, significantly less glucose-6-phosphate is converted to fructose-6-phosphate by the phosphoglucoisomerase than in the absence of the organic acids (54.8\% compared to $64.4 \%$ relative to the total amount of glucose) (Fig. 2.1). Similarly, relatively less glucose-6-phosphate is used for biosynthetic purposes under these conditions. This might result from the availability of glutamate and succinate as precursors for many amino acids that have to be produced from the glucose in CG but not in CGSE medium. In contrast, the portion of glucose-6-phosphate that is oxidized by glucose-6-phosphate dehydrogenase is significantly increased (43.7\% compared to $33.2 \%)$. This regulation of glucose-6-phosphate partition is not reflected by the transcriptional regulation of the genes encoding the two major enzymes that use glucose-6-phosphate as a substrate, i.e., pgi and $z w f$, which are constitutively expressed under the conditions used here. Previous studies indicated that the expression of these genes is also not affected by the presence of glucose in the medium (Blencke et al., 2003; Ludwig et al., 2001). A candidate that might cause the increased flux through the pentose phosphate pathway in the presence of succinate and glutamate is the $y w l F$ gene, tentatively believed to encode ribulose-5-phosphate epimerase. The expression of this gene increased weakly but significantly upon the addition of the organic acids (Fig. 2.2). Moreover, both the transketolase (Tkt) and transaldolase (YwjH) were recently shown to be phosphorylated in vivo in B. subtilis (Lévine et al., 2006). It is, however, unknown under which conditions these phosphorylations occur and how they affect the activities of the two enzymes. Due to the convergence of the pentose phosphate pathway with glycolysis at the fructose-6-phosphate and glyceraldehyde-3-phosphate levels, the changes in the partition of glucose-6-phosphate do not affect the fluxes through the lower part of glycolysis, which are essentially the same in both media (see above; Fig. 2.1). 


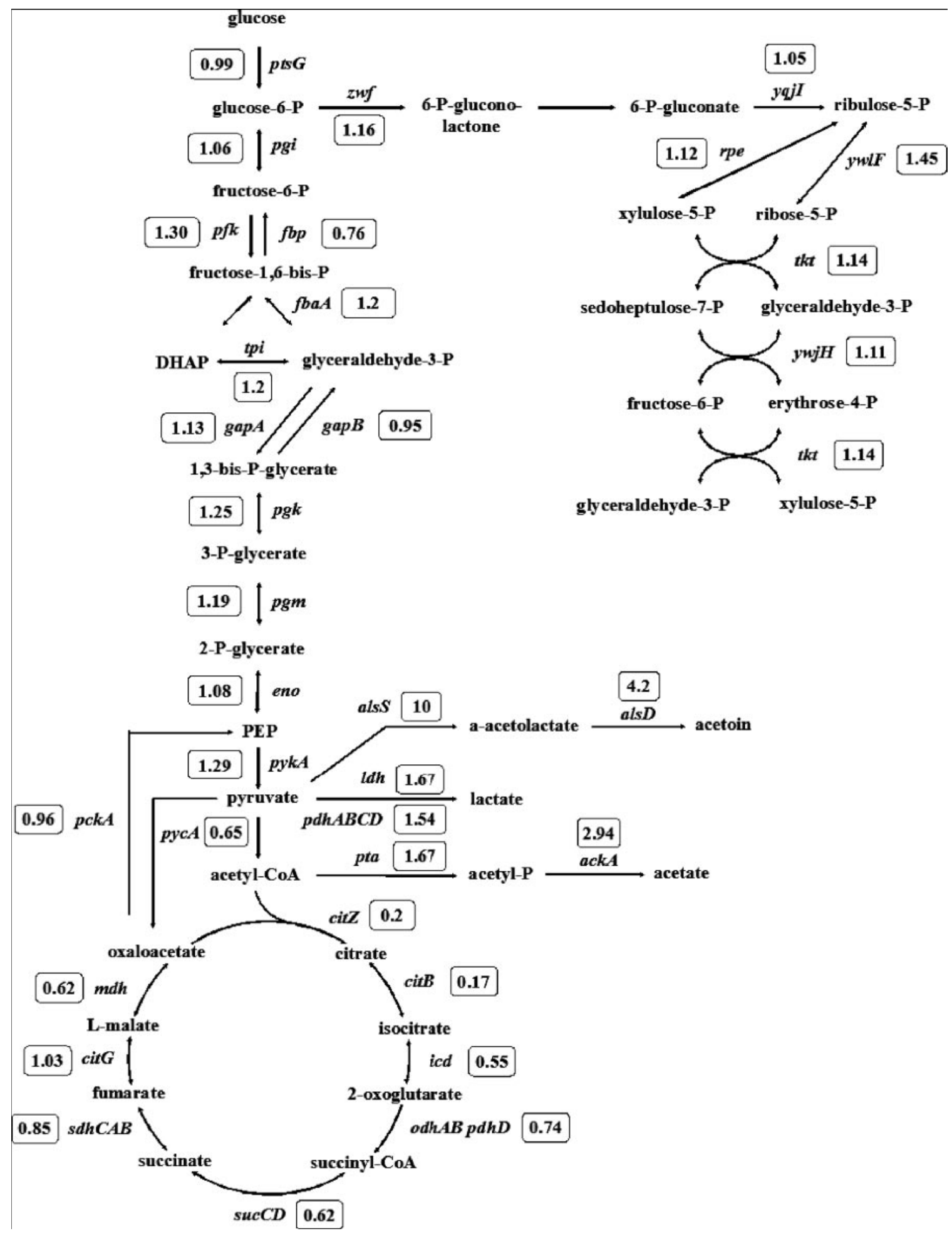

Figure 2.2. Overview on the regulation of genes encoding enzymes of glycolysis, the pentose phosphate shunt, and the Krebs cycle in B. subtilis. The result of the transcriptome analysis is given next to each gene. The numbers correspond to the ratios of gene expression under the two conditions (CGSE/CG). For multisubunit enzymes encoded by operons, the mean factors of regulation of the genes of the operon are shown. A complete list of all studied genes is given elsewhere (see the supplemental material). PEP, phosphoenolpyruvate; DHAP, dihydroxyacetone phosphate. 


\section{The partition of pyruvate and acetyl-CoA and the regulation of overflow metabolism}

Pyruvate is among the most versatile metabolic intermediates. It can be oxidized to generate acetyl coenzyme A (acetyl-CoA) to enter the Krebs cycle (pyruvate dehydrogenase complex; $p d h A B C D$ ), it can be converted to acetoin (acetolactate synthase and acetolactate decarboxylase; als $S$ and als $D$, respectively), it can be reduced for the regeneration of $\mathrm{NAD}^{+}$ (lactate dehydrogenase; $l d h$ ), and it can be used as an acceptor of carbon dioxide to replenish the Krebs cycle (pyruvate carboxylase; $p y c A$ ). Moreover, the synthesis of several amino acids requires pyruvate as a precursor. Finally, pyruvate can be excreted into the medium.

The transcriptome data revealed that expression of the pyruvate dehydrogenase complex $(p d h A B C D)$ is weakly but significantly increased in the presence of the organic acids. This observation is supported by an assay of the $\beta$-galactosidase activity driven by the $p d h A$ promoter (Table 4). However, this increase is not paralleled by an increased formation of acetyl-CoA from pyruvate in the presence of glutamate and succinate (probably due to the high activity of lactate dehydrogenase under these conditions [see below]).

Acetyl-CoA can be transferred to oxaloacetate to initiate the Krebs cycle (citrate synthase [CitZ]) for substrate level phosphorylation (generation of acetate involving phosphotransacetylase Pta and acetate kinase AckA [see below]) or for biosynthetic purposes. In the presence of glucose, B. subtilis is known to excrete large amounts of acetate. Indeed, significant acetate production was observed for CG medium. This acetate production was even strongly increased if glutamate and succinate were present (CGSE medium) (Fig. 2.1). This increased acetate excretion corresponds well with the increased transcription of the pta and $a c k A$ genes, encoding phosphotransacetylase and acetate kinase, respectively, as detected in the transcriptome analysis. Finally, the pta promoter was found to be stimulated fourfold by the presence of glutamate and succinate (Table 4). In contrast, the flux of acetyl-CoA into the tricarboxylic acid branch of the Krebs cycle was severely reduced in the presence of glutamate and succinate (Fig. 2.1). The reduction of citrate formation from acetyl-CoA is probably due to the fivefold reduced expression of $c i t Z$, encoding the major citrate synthase of B. subtilis in CGSE medium. Since the citrate synthase works according to the MichaelisMenten kinetics, the fivefold repression in citZ expression results in a fivefold reduced flux of acetyl-CoA into the Krebs cycle. This may reflect the lack of need of the Krebs cycle if glucose, as a source of carbon and energy, and glutamate and succinate, as Krebs cycle intermediates, are available for the cell.

As long as glucose is present in excess, B. subtilis produces acetate to benefit from the additional substrate-level phosphorylation. However, the accumulation of acetate results in (i) 
the acidification of the medium (Tobisch et al., 1999b) and (ii) the induction of the alsSD operon, encoding acetolactate synthase and acetolactate decarboxylase, and the subsequent synthesis of acetoin (Cruz Ramos et al., 2000). It is not known whether the decrease of the $\mathrm{pH}$ or the accumulation of acetate triggers AlsR, the transcription activator of the als $S D$ operon. Our microarray study revealed that transcription of the als $S D$ operon is strongly induced by the presence of glutamate and succinate (10-fold induction of alsS) (Fig. 2.2). In this experiment, the $\mathrm{pH}$ value of the culture medium decreased from 7.17 to 7.01 on $\mathrm{CG}$ and from 7.33 to 7.04 on CGSE. The nearly constant $\mathrm{pH}$ of the culture medium suggests that the accumulation of acetate is the primary trigger of AlsR activity. However, no excretion of acetoin was observed under either condition. This may be due to the fact that acetoin might have just started to accumulate and thus been below the detection limit even if the genes were actively transcribed. Alternatively, the translation or the activity of acetolactate synthase and/or acetolactate decarboxylase might be controlled at an additional level. Indeed, the phosphorylation of acetolactate decarboxylase was recently reported (Lévine et al., 2006). The expression of the $l d h$ gene encoding lactate dehydrogenase is induced more weakly in CGSE medium than in CG medium. However, no excretion of lactate was detected in CG medium, whereas significant amounts of lactate were detected in the medium of the culture grown in the presence of glutamate and succinate.

If glucose is provided as the only carbon source (as in CG medium), the growth of B. subtilis depends on the activity of pyruvate carboxylase to replenish the Krebs cycle. Indeed, a significant portion of pyruvate is used for oxaloacetate production. In the presence of glutamate and succinate, the anaplerotic reactions are less important. This idea is supported by the finding that the flux of pyruvate to oxaloacetate is reduced about threefold in CGSE medium. This reduced flux is paralleled by a reduced expression of the $p y c A$ gene, which encodes pyruvate carboxylase, in CGSE medium relative to what is seen for CG medium. However, the regulation of $p y c A$ expression is much weaker than that of the flux through the reaction catalyzed by the pyruvate carboxylase. Thus, additional factors that control the activity of the enzyme may be involved. In both media, an important portion of pyruvate (about 12 to $15 \%$ of the total pyruvate pool) is used for the production of biomass. Finally, a small amount of pyruvate is excreted into the medium. Excretion is increased fourfold in CGSE medium; however, the proteins and gene products involved in pyruvate excretion have not yet been identified. 


\section{Regulation of the Krebs cycle}

The Krebs cycle is central to the physiology of most heterotrophic organisms because it generates reducing power for respiration, precursors for anabolism, and an additional ATP in substrate-level phosphorylation. In B. subtilis, the expression of the genes encoding the initial enzymes of the Krebs cycle, citrate synthase and aconitase (citZ and citB, respectively), is synergistically repressed by glucose and glutamate/ammonium (Blencke et al., 2006; JourlinCastelli et al., 2000). Our transcriptome analysis confirmed this regulatory pattern: both genes were repressed fivefold in CGSE medium (Fig. 2.2). Moreover, the activity of the citB promoter was repressed about tenfold by the presence of the organic acids in addition to glucose (Table 4). This repression of initial enzymes of the Krebs cycle is paralleled by the 4.6-fold reduction of carbon flow from acetyl-CoA to 2-oxoglutarate in CGSE from the level seen for CG medium. In the presence of externally provided glutamate and succinate, these acids can be taken up and glutamate can be converted to 2-oxoglutarate. As a result, the flux from glutamate to oxaloacetate was higher in CGSE than in CG medium. The genes of the enzymes catalyzing the reactions from 2-oxoglutarate to oxaloacetate were weakly regulated by the presence of organic acids. This reflects the need for these enzymes under both conditions studied in this work.

The genes encoding the transporters for succinate and glutamate, $\operatorname{dct} P, \operatorname{glt} P$, and $\operatorname{glt} T$, are only weakly affected by the presence of organic acids. It should be noted, however, that $d c t P$ and $g l t P$ are subject to a catabolite repression in the presence of glucose (Blencke et al., 2003 and our unpublished results), suggesting that the two major transporters for succinate and glutamate are relatively weakly expressed under both conditions addressed in this study. In any case, significant amounts of glutamate and succinate were transported into the cells if these acids were available.

\section{Regulation of nitrogen assimilation}

The basic $\mathrm{C}$ medium used in this study contained ammonium, which can be transported into the cell by the $\operatorname{NrgA}(\mathrm{AmtB})$ transport protein and assimilated by the glutamine synthetase/glutamate synthase cycle made up of glutamine synthetase and glutamate synthase. In the presence of glutamate, neither the ammonium transporter nor the glutamate synthase is required for growth. The determination of the $n r g A$ promoter activity demonstrated that the gene was not expressed in the two media studied here (Table 4). This is due to the lack of transcription activation at high ammonium concentrations, which allow the entry of ammonia by diffusion (Detsch and Stülke, 2003). Similarly, the transcriptome data revealed that $n \operatorname{rg} A$ 
expression was not affected by the presence of glutamate. The expression of $g \ln A$ was slightly repressed in CGSE medium. This may result from the reduced need for glutamine synthesis if glutamate is present in the medium: under these conditions, glutamine synthesis is required only to meet the need for this amino acid, while it is an intermediate in glutamate biosynthesis in CG medium. The gltAB operon, encoding the two subunits of glutamate synthase, was repressed about twofold by the addition of glutamate and succinate, as judged from both transcriptome and promoter fusion data (Table 4). Accordingly, no conversion of 2-oxoglutarate to glutamate was detectable in CGSE medium. The imported glutamate was used for biomass production (about $25 \%$ of the glutamate), and the remaining $75 \%$ was converted to 2-oxoglutarate by the action of glutamate dehydrogenase encoded by rocG. Interestingly, $\operatorname{roc} G$ expression was somewhat lower in the presence of glutamate and succinate. However, it should be mentioned that only basal $\operatorname{roc} G$ expression occurs in both $\mathrm{CG}$ and CGSE media, since the expression of $\operatorname{roc} G$ requires induction by arginine and moreover is repressed by glucose (Belitsky and Sonenshein, 1998). Obviously, this low expression of glutamate dehydrogenase is sufficient to make a substantial contribution to feed the Krebs cycle in CGSE-grown bacteria. Thus, even though only weakly regulated at the level of transcription, the activities of the two enzymes interconverting 2-oxoglutarate and glutamate are mutually absolutely exclusive.

\section{Conclusion}

The metabolism of $B$. subtilis has evolved to adapt to a broad range of conditions of nutrient supply. This adaptation often involves changes in the transcription of the relevant genes. However, the $l d h$ gene, encoding lactate dehydrogenase, is constitutively transcribed under the conditions studied here, even though lactate formation in CGSE medium is strongly increased compared to that in CG medium. In this case, the enzymatic activity of lactate dehydrogenase might be controlled, perhaps by the intracellular redox state. This suggestion is in good agreement with the observation that the expression of the ldh gene is controlled by the redox state of the cell in the presence of nitrate (Reents et al., 2006). On the other hand, some transcriptional regulation events are not paralleled by changes in the carbon fluxes. This was observed for the alsSD operon, required for acetoin biosynthesis. In this case, posttranslational control of AlsD activity by protein phosphorylation might account for an additional level of regulation. Phosphorylation is a common means to adjust protein activities to the metabolic requirements. However, only with the advance of proteomic methods can 
these modifications be detected (Lévine et al., 2006). The role of protein phosphorylation in the control of metabolic pathways is well documented in the case of E. coli isocitrate dehydrogenase, and this phosphorylation also occurs in B. subtilis (El-Mansi et al., 2006; Lévine et al., 2006). Moreover, the control of 2-oxoglutarate dehydrogenase activity by phosphorylation was recently discovered for Corynebacterium glutamicum (Niebisch et al., 2006). Thus, much work remains to be done to study the details of the regulatory events that occur at the different stages after transcription involving changes in mRNA stability, differential translation, and posttranslational modifications. This work will be essential for the development of systems biology. 


\section{Multiple-mutation reaction: A method for the simultaneous introduction of multiple mutations into the glpK gene of Mycoplasma pneumoniae}

The results described in this chapter were published in:

Hames, C., S. Halbedel, O. Schilling, and J. Stülke. 2005. Multiple-mutation reaction: a method for simultaneous introduction of multiple mutations into the $g l p K$ gene of Mycoplasma pneumoniae. Appl Environ Microbiol 71:4097-4100.

Author contributions:

This study was designed by J.S., S.H. and O.S.. O.S. established the basic principles of the method. C.H. performed all experiments. S.H. designed the oligonucleotides and supervised the experimental work that was accomplished by C.H. during her diploma thesis. All authors were involved in the interpretation of the collected data and in the writing of the manuscript. 


\begin{abstract}
In Mycoplasma pneumoniae, the UGA opal codon specifies tryptophan rather than a translation stop site. This often makes it difficult to express Mycoplasma proteins in E. coli isolates. In this work, we developed a strategy for the one-step introduction of several mutations. This method, the multiple-mutation reaction, is used to simultaneously replace nine opal codons in the M. pneumoniae glpK gene.
\end{abstract}

\title{
Introduction
}

Mycoplasma pneumoniae is a pathogen that lives on mucosal surfaces and causes diseases such as mild pneumonia, tracheobronchitis, and complications affecting the central nervous system, the skin, and mucosal surfaces (Jacobs, 1997; Lind, 1983; Waites and Talkington, 2004). This bacterium possesses one of the smallest genomes of any free-living organism known so far. This reduced genome makes Mycoplasma spp. interesting from two points of view: (i) the analysis of these bacteria may help to identify the minimal set of genes that is required for independent life (Hutchison III et al., 1999), and (ii) M. pneumoniae and its close relative $M$. genitalium are well suited for the development of the methods of the postgenomic era (Jaffe et al., 2004; Wasinger et al., 2000). Another interesting aspect of the small genome is the observation that several enzymes of Mycoplasma spp. are "moonlighting"; i.e., they have multiple unrelated functions (Jeffery, 1999). This was discovered for glycolytic kinases, which are also active as nucleoside diphosphate kinases in M.pneumoniae and other Mycoplasma spp. (Pollack et al., 2002).

However, the analysis of proteins from Mycoplasma spp. is hampered by a peculiarity of the genetic code of these bacteria: they use the UGA opal codon to incorporate tryptophan rather than as a stop codon as in the universal genetic code (Inamine et al., 1990; Simoneau et al., 1993). Thus, if cloned into Escherichia coli or other hosts, the genes from M. pneumoniae may contain many stop codons that prevent heterologous expression. Several strategies have been developed to solve this problem. For example, some $M$. pneumoniae genes, such as $p t s H$ or $h p r K$, do not possess UGA codons and thus require no special care (Steinhauer et al., 2002). Expression of mollicute genes in Spiroplasma spp. that read the UGA as a tryptophan codon was reported, but these bacteria are difficult to handle (Stamburski et al., 1991). E. coli suppressor strains expressing an opal suppressor tRNA were developed, but they fail if 
multiple opal codons are present (Smiley and Minion, 1993). M. pneumoniae genes containing few UGA codons have been expressed in Bacillus subtilis with low efficiency (Kannan and Baseman, 2000). In cases with only a few opal codons, these were changed by site-directed mutagenesis to allow expression in E. coli (Knudtson et al., 1997; Noh et al., 2002). The M. pneumoniae P1 adhesin gene contains 21 opal codons, and a large-scale purification of the protein, though highly desired, has so far not been possible. In this case, protein fragments were expressed and purified (Chaudhry et al., 2005). Finally, Mycoplasma genes could be synthesized in vitro from oligonucleotides; this strategy is, however, quite expensive. In this work, we present a PCR-based method that allows the simultaneous introduction of several mutations in a single step. Using this strategy, 9 of the 10 opal codons of the $g l p K$ gene from $M$. pneumoniae were modified, leading to expression of glycerol kinase in E. coli.

\section{Outline of the multiple-mutation reaction (MMR) strategy}

Several methods for PCR-based site-directed mutagenesis have been developed. Among these, the combined chain reaction method (Bi and Stambrook, 1997; Bi and Stambrook, 1998) proved to be very rapid and reliable. The principle of this method is the use of mutagenic primers that hybridize more strongly to the template than the external primers. The mutagenic primers are phosphorylated at their $5^{\prime}$ ends, and these are ligated to the $3^{\prime} \mathrm{OH}$ groups of the extended upstream primers by the action of a thermostable DNA ligase. Moreover, the DNA polymerase employed must not exhibit $5^{\prime} \rightarrow 3^{\prime}$ exonuclease activity, to prevent the degradation of the extended primers. In our view, $P f u$ and $P w o$ polymerases are both well suited (Meinken et al., 2003; Schilling et al., 2004). The original protocol describes the introduction of two mutations simultaneously. In a previous study, we used a combined chain reaction to mutagenize four distant bases in a DNA fragment in a one-step reaction (our unpublished results).

For the introduction of up to nine mutations in a single experiment, we developed the MMR. This method requires the efficient binding of all the mutagenic primers to the target DNA. To ensure that extension of a PCR product is not possible beyond the next (i.e., more downstream) mutation site without ligation to the corresponding mutagenic primer, special care needs to be taken in primer design. This reaction is based on an accurate calculation of melting temperatures. For this purpose, the formula $T_{m}$ (melting temperature in ${ }^{\circ} \mathrm{C}$ ) $=81.5+$ $16.6(\log [\mathrm{Me}+])+0.41 \times \% \mathrm{G}+\mathrm{C}-(500 /$ oligonucleotide length $)-0.61 \times \%$ formamide was used (Meinkoth and Wahl, 1984). Only bases that match between primer and template were 
used for the calculation. One consideration was made when designing the mutagenic primers: ligation was facilitated by placing a $\mathrm{G}$ or $\mathrm{C}$ at the $5^{\prime}$ end of the oligonucleotide to favor close duplex formation between the primer and the target DNA.

Table 5. Oligonucleotides used in this study

\begin{tabular}{|c|c|c|c|}
\hline Oligonucleotide & Sequence $\left(5^{\prime}-3^{\prime}\right)^{\mathrm{a}}$ & Mutation & $T_{m}\left({ }^{\circ} \mathrm{C}\right)$ \\
\hline $\mathrm{CH} 7$ & $\begin{array}{l}\text { AAAAGTCGACATGGATCTAAAACAACAATAC } \\
\text { ATTCTTG }\end{array}$ & None & 59 \\
\hline $\mathrm{CH} 8$ & $\begin{array}{l}\text { TATAAAGCTTGTCTTAGTCTAAGCTAGCCCAT } \\
\text { TTTAG }\end{array}$ & A1512G & 63 \\
\hline $\mathrm{CH} 9$ & AAAAGTCGACATGGATCTAAAACAAC & None & 57 \\
\hline $\mathrm{CH} 10$ & TATAAAGCTTGTCTTAGTCTAAGCTAG & None & 59 \\
\hline $\mathrm{CH} 11$ & P-GATCCCTTAGAAATTTGGTCAGTCCAATTAG & A165G & 64 \\
\hline $\mathrm{CH} 12$ & $\begin{array}{l}\text { P-CCATTGTGTTATGGAACAAAGAAAATGGTT } \\
\text { TG }\end{array}$ & A273G & 63 \\
\hline $\mathrm{CH} 13$ & $\begin{array}{l}\text { P-CACTAAGATTGCTTGGATCTTGGAAAATGTT } \\
\text { C }\end{array}$ & A438G & 64 \\
\hline $\mathrm{CH} 14$ & P-CCTGGTTAATTTGGAAACTAACGGGTG & $\mathrm{A} 522 \mathrm{G}$ & 64 \\
\hline $\mathrm{CH} 15$ & P-CCATGACATGGTCACAAGAGTTAGGC & A606G & 65 \\
\hline $\mathrm{CH} 16$ & P-TACCGAGTCATTGGTCTACTAGTGC & A705G & 65 \\
\hline $\mathrm{CH} 17$ & P-CCTTAAAGTGGTTAAGGGATAGTCTTAAGG & A966G & 65 \\
\hline $\mathrm{CH} 18$ & $\begin{array}{l}\text { P-GCAGTTAATTATTGGAAGGACACTAAACAA } \\
\text { C }\end{array}$ & A1386G & 63 \\
\hline $\mathrm{CH} 19$ & P-GAAATCAAAGCGTTGGAACGAAGCTG & $\mathrm{A} 1482 \mathrm{G}$ & 65 \\
\hline
\end{tabular}

${ }^{a}$ The "P" at the 5 ' end of oligonucleotide sequences indicates phosphorylation.

The external primers were selected to have melting temperatures considerably lower (about $4{ }^{\circ} \mathrm{C}$ ) than those of the mutagenic primers. The MMR was performed with 2.5 units of Pfu DNA polymerase (MBI Fermentas, Lithuania) and 15 units of Ampligase ${ }^{\circledR}$ (Epicentre, Madison, WI) in MMR buffer (20 mM Tris- $\mathrm{HCl}$ [pH 8.5], $3 \mathrm{mM} \mathrm{MgCl}_{2}, 50 \mathrm{mM} \mathrm{KCl}$, $0.4 \mathrm{mg} / \mathrm{ml}$ bovine serum albumin, and $0.5 \mathrm{mM} \mathrm{NAD}^{+}$) in a total volume of $50 \mu 1$. Conditions for MMR included denaturation at $95^{\circ} \mathrm{C}$ for $30 \mathrm{~s}$, primer annealing at $57^{\circ} \mathrm{C}$ for $30 \mathrm{~s}$, and elongation at $65^{\circ} \mathrm{C}$ for $6 \mathrm{~min}$, for 35 cycles. Initially, the DNA fragment (100 ng) was denatured for $5 \mathrm{~min}$ at $95{ }^{\circ} \mathrm{C}$. Ten picomoles of each primer were used. The sequences and the arrangements of the oligonucleotides used in this study are shown in Table 5 and Figure 3.1, respectively. 


\section{Cloning of M. pneumoniae glpK and expression of the protein in E. coli}

An analysis of growth behavior and the in vivo protein phosphorylation pattern identified glycerol as a key carbon source associated with regulatory phenomena. This substrate triggered in vivo phosphorylation of the HPr protein of the phosphotransferase system by the metabolite-sensitive HPr kinase/phosphorylase (Halbedel et al., 2004; Steinhauer et al., 2002). We were therefore interested in studying glycerol metabolism and its regulation in more detail. As a first step, we intended to purify the glycerol kinase. This enzyme is known to be a key target of catabolite regulation in Gram-positive bacteria (Darbon et al., 2002; Stülke and Hillen, 2000). However, the corresponding glpK gene contains 10 opal codons and was therefore a good subject for MMR in order to change these codons to tryptophan codons for E. coli. The glpK gene was amplified using the oligonucleotides $\mathrm{CH} 7$ and $\mathrm{CH} 8$ and chromosomal DNA of M. pneumoniae M129 (ATCC 29342) as a template. With CH8, the most C-terminal opal codon was replaced by a TGG codon. The amplicon was cloned between the SalI and HindIII sites of the expression vector pWH844 (Schirmer et al., 1997). The resulting plasmid, pGP253, was used as a template for MMR with $\mathrm{CH} 9$ and $\mathrm{CH} 10$ as external primers and $\mathrm{CH} 11$ through $\mathrm{CH} 19$ as mutagenesis primers. Five independent MMRs were carried out, and the MMR products were individually cloned as a SalI/HindIII fragment into pWH844. The inserts of one clone resulting from each MMR were sequenced. Out of the five candidates, three contained the nine desired mutations without any additional mutations. One plasmid contained seven out of nine mutations, and the fifth plasmid bore all nine mutations and one additional undesired 1-bp deletion in one of the primer regions.

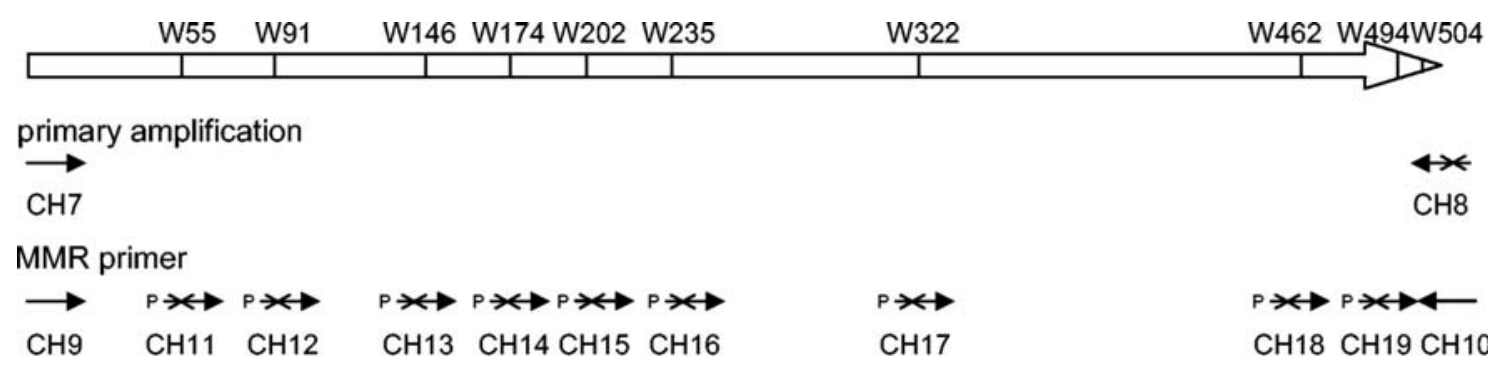

Figure 3.1. Strategy for amplification and mutagenesis of the $M$. pneumoniae glpK gene (MPN050 (Himmelreich et al., 1996)). The positions of the opal codons in the wild type glpK gene (indicated by a $\mathrm{W}$ followed by the number corresponding to the amino acid) and the position and orientation of the external and mutagenic oligonucleotides are shown. The annealing site of each oligonucleotide is indicated by an arrow. Oligonucleotides bearing an $A \rightarrow G$ transition are depicted by crossed arrows. 
Plasmids bearing all nine desired mutations but no additional mutations were designated pGP254. pGP254 allows the expression of M. pneumoniae glycerol kinase fused to an Nterminal hexahistidine sequence under the control of an isopropyl- $\beta$-D-thiogalactopyranoside (IPTG)-inducible promoter. To test the success of the mutagenesis, we compared the protein contents of E. coli cultures carrying either pWH844, pGP253, or pGP254.
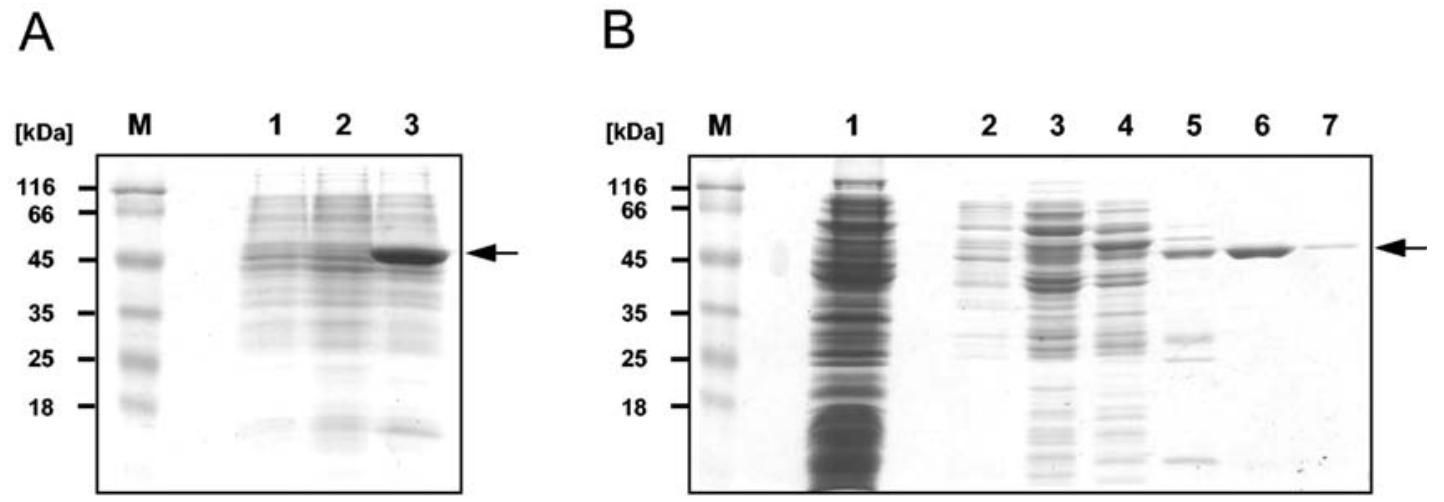

Figure 3.2. Overproduction and purification of $M$. pneumoniae GlpK. (A) Sodium dodecyl sulfate (SDS)-polyacrylamide gel electrophoresis for the detection of His $_{6}$-tagged GlpK in crude extracts of E. coli DH5 $\alpha$ bearing either the empty expression vector pWH844 (lane 1), the expression vector including the wild type $g l p K$ allele, pGP253 (lane 2), or the vector including the mutated $g l p K$ allele, pGP254 (lane 3). Cells were grown to an optical density at $600 \mathrm{~nm}$ of 0.8 , and expression from the IPTG-inducible promoter was induced by addition of $1 \mathrm{mM}$ IPTG (final concentration). After $2 \mathrm{~h}$, cells were harvested and disrupted by sonication. The insoluble fraction was pelleted in a centrifugation step and solubilized using $6 \mathrm{M}$ urea, and sample aliquots were separated on an SDS$12 \%$ polyacrylamide gel. (B) SDS-polyacrylamide gel electrophoresis to monitor the purification of His $_{6}$-tagged GlpK. Crude extract of the GlpK expression strain (E. coli DH5 $\alpha$ bearing the plasmid pGP254) that had been grown in the presence of $1 \mathrm{mM}$ IPTG was passed over a $\mathrm{Ni}^{2+}$-nitrilotriacetic acid superflow column ( $5 \mathrm{ml}$ bed volume; QIAGEN) and washed extensively with a buffer containing $10 \mathrm{mM}$ Tris- $\mathrm{HCl}$ ( $\mathrm{pH} 7.4$ ) and $200 \mathrm{mM} \mathrm{NaCl}$, followed by elution with an imidazole gradient (from 10 to $500 \mathrm{mM}$ imidazole). Aliquots of the individual fractions were separated on SDS- $12 \%$ polyacrylamide gels. A prestained protein molecular mass marker (Fermentas) served as a standard (lane M). Lane 1, flowthrough; lane 2, $10 \mathrm{mM}$ imidazole; lane 3, $20 \mathrm{mM}$ imidazole; lane 4, $50 \mathrm{mM}$ imidazole; lane 5, $100 \mathrm{mM}$ imidazole; lane 6, $200 \mathrm{mM}$ imidazole; lane 7, $500 \mathrm{mM}$ imidazole.

A prominent band corresponding to an approximate molecular mass of $56 \mathrm{kDa}$ is detectable in the strain bearing pGP254, while no such protein is expressed from pGP253 encoding the unmutated glpK gene (see Fig. 3.2A). The glycerol kinase was purified to apparent homogeneity by $\mathrm{Ni}^{2+}$-nitrilotriacetic acid chromatography as described previously (see Fig. 3.2B) (Meinken et al., 2003). Thus, MMR was successful in achieving efficient overproduction of M. pneumoniae glycerol kinase for biochemical studies. 
This study demonstrates that MMR can be used for the rapid and highly efficient introduction of multiple mutations into a gene. Four out of five individual clones had the desired mutations. Of these four, only one candidate contained an extra mutation, which was most probably due to an impure oligonucleotide mix. Indeed, other experiments indicated that the quality of the oligonucleotides is the limiting factor for MMR. Obviously, this method is useful not only for the expression of Mycoplasma species genes, but also to change codon usage patterns or for any other purpose that requires the introduction of many mutations or combinations of mutations at the same time. What is the maximum number of mutations that can be introduced by MMR in a single step? Our results suggest that the target of nine mutations is still far from a theoretical limit, and we are confident that this method can be made even more effective by taking care of the quality of the oligonucleotides (see above) and by using mutagenic primers that alternate between the two strands of the DNA. With this method at hand, even the expression of a functional P1 adhesin gene in E. coli, which has so far been beyond imagination (Chaudhry et al., 2005), now seems feasible. 


\section{A protein-dependent riboswitch controlling ptsGHI operon expression in Bacillus subtilis: RNA structure rather than sequence provides interaction specificity}

The results described in this chapter were published in:

Schilling, O., I. Langbein, M. Müller, M. H. Schmalisch, and J. Stülke. 2004. A proteindependent riboswitch controlling ptsGHI operon expression in Bacillus subtilis: RNA structure rather than sequence provides interaction specificity. Nucleic Acids Res 32:28532864.

\section{Author contributions:}

This study was designed by O.S., I.L., M.H.S., and J.S.. O.S. performed and interpreted all reporter gene analyses and most of the gel shift experiments. O.S. constructed all mutant strains from GP366 to GP392. I.L. perfomed the RNase T1 footprint analysis and one of the gel shift experiments. I.L. constructed the mutant strains from GP150 to GP191. M.M. constructed the GlcT-RBD overexpression vector pGP230. M.H.S. supervised O.S. in his diploma work. O.S. and J.S. wrote the paper. 


\begin{abstract}
The Gram-positive soil bacterium Bacillus subtilis transports glucose by the phosphotransferase system. The genes for this system are encoded in the ptsGHI operon. The expression of this operon is controlled at the level of transcript elongation by a proteindependent riboswitch. In the absence of glucose a transcriptional terminator prevents elongation into the structural genes. In the presence of glucose, the GlcT protein is activated and binds and stabilizes an alternative RNA structure that overlaps the terminator and prevents termination. In this work, we have studied the structural and sequence requirements for the two mutually exclusive RNA structures, the terminator and the RNA antiterminator (the RAT sequence). In both cases, the structure seems to be more important than the actual sequence. The number of paired and unpaired bases in the RAT sequence is essential for recognition by the antiterminator protein GlcT. In contrast, mutations of individual bases are well tolerated as long as the general structure of the RAT is not impaired. The introduction of one additional base in the RAT changed its structure and resulted in complete loss of interaction with GlcT. In contrast, this mutant RAT was efficiently recognized by a different B. subtilis antitermination protein, LicT.
\end{abstract}

\title{
Introduction
}

In bacteria, gene expression is most commonly regulated at the level of transcription initiation. This control is achieved by interactions of specific DNA sequences with regulatory proteins. A second mechanism of control of gene expression is mediated at the RNA level by riboswitches. This type of control may affect either transcription or translation. The formation of riboswitches can be triggered by low molecular weight effectors, tRNAs or by regulatory proteins (for reviews see Henkin, 2000; Stülke, 2002; Winkler and Breaker, 2003).

Regulatory protein-RNA interactions control the expression of genes involved in diverse physiological functions. In Bacillus subtilis and other Gram-positive bacteria, genes encoding enzymes for the biosyntheses of tryptophan and pyrimidines are controlled by a termination/antitermination mechanism (Babitzke and Gollnick, 2001; Switzer et al., 1999). Genes required for the utilization of several sugars are subject to regulation by antitermination in many bacteria (Rutberg, 1997). Similarly, the utilization of aliphatic amides in Pseudomonas aeruginosa and assimilatory nitrate reduction in Klebsiella oxytoca is 
controlled by RNA-binding antitermination proteins (Chai and Stewart, 1998; Wilson et al., 1996). Recently, an antitermination mechanism that controls gene expression after a cold shock in Escherichia coli was discovered (Bae et al., 2000).

As with all other riboswitches, protein-dependent antitermination/termination systems are based on the existence of alternative, mutually exclusive RNA structures. One of these structures is a transcriptional terminator. The alternative structure, also called the RNA antiterminator (RAT) (Aymerich and Steinmetz, 1992), prevents formation of the terminator and allows transcription elongation to proceed. Depending on the nature of the system, one of the structures is energetically favored and can form in the absence of any other factor. In contrast, the less favored structure depends on stabilization, which is caused by binding of the regulatory protein. While the antiterminators are more stable in the anabolic systems resulting in transcription elongation as the default state, formation of the terminators is energetically favored in many catabolic systems. Thus, the respective genes are not expressed unless the inducer is present and binding of the antiterminator protein allows formation of the antitermination structure (Aymerich and Steinmetz, 1992; Henkin, 2000; Stülke, 2002).

We are interested in the control of glucose utilization in B. subtilis. Glucose is the preferred source of carbon and energy for these bacteria. The sugar is transported by the bacterial sugar:phosphoenolpyruvate phosphotransferase system (PTS) and is subsequently catabolized in the glycolytic and pentose phosphate pathways (for a review see Stülke and Hillen, 2000). Two operons encoding enzymes of glucose catabolism are inducible by the presence of glucose in the medium, the ptsGHI operon and the gapA operon coding for the components of the PTS and for glycolytic enzymes of triose phosphate interconversions, respectively (Ludwig et al., 2001; Stülke et al., 1997; Tobisch et al., 1999b). While induction of the gapA operon is governed at the level of transcription initiation by a specific repressor (Doan and Aymerich, 2003; Fillinger et al., 2000; Ludwig et al., 2001), the ptsGHI operon is controlled by transcriptional antitermination (Stülke et al., 1997). Transcription of this operon is constitutively initiated but stops at a factor-independent terminator upstream of the ptsG structural gene. In the presence of glucose, an antiterminator protein, GlcT, is activated and is thought to bind a RAT sequence overlapping the transcription terminator (see Fig. 4.1). GlcT binding to the RAT prevents formation of the terminator and allows transcription elongation (Langbein et al., 1999). The RNA-binding activity of GlcT is controlled by reversible phosphorylation in response to the presence of the inducer, glucose. 


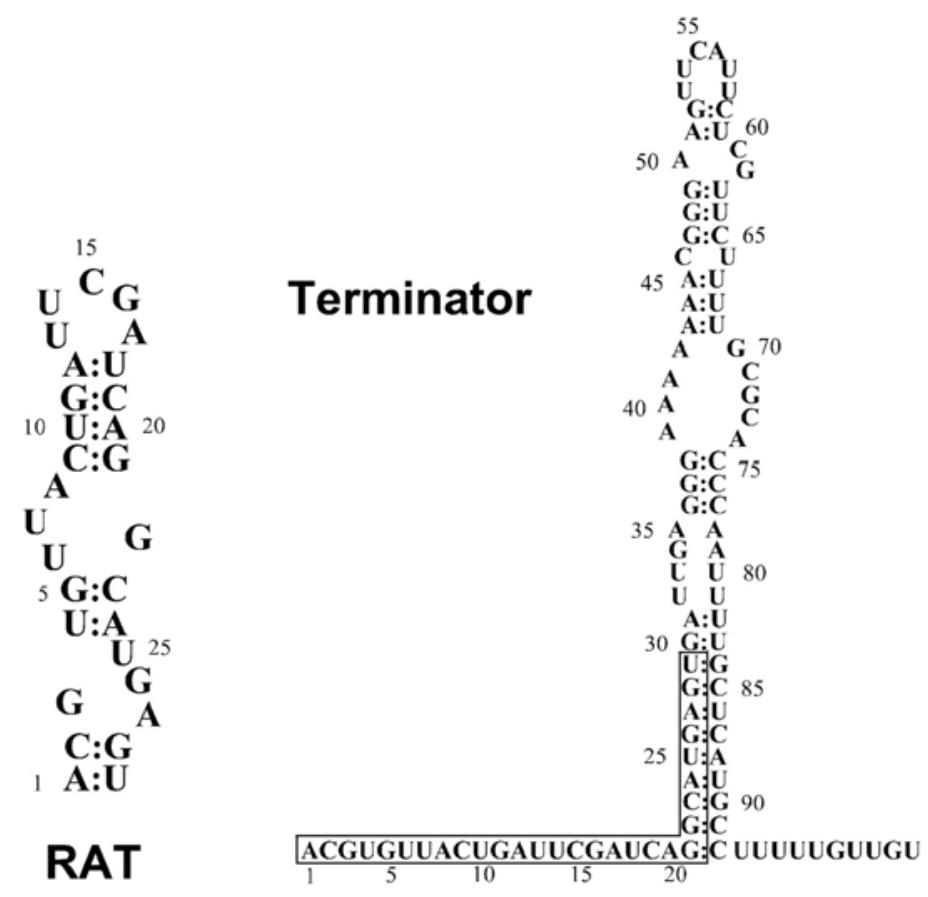

Figure 4.1. Proposed schematic model of the antiterminator and terminator structures of the ptsG leader mRNA. The secondary structure of the antiterminator is based on known RAT structures (Yang et al., 2002). The RAT sequence is boxed in the terminator structure to highlight the overlap between the RAT and the terminator that constitutes the riboswitch.

In the presence of glucose, the glucose-specific enzyme II of the PTS may transfer its phosphate group to the sugar. In contrast, enzyme II is permanently phosphorylated in the absence of glucose and this form may phosphorylate and thereby inactivate the antiterminator protein GlcT (Bachem and Stülke, 1998; Schmalisch et al., 2003).

GlcT is a member of a family of transcriptional antiterminators, the prototypes of which are BglG from E. coli and SacY and LicT from B. subtilis. These proteins are all composed of an N-terminal RNA-binding domain (Langbein et al., 1999; Manival et al., 1997) and two reiterated PTS regulation domains (PRDs) which control the RNA-binding activity of the antiterminators via phosphorylation-dependent dimerization (Amster-Choder and Wright, 1992; Fux et al., 2003; Stülke et al., 1998; Tortosa et al., 1997). The RNA sequences bound by these antiterminators are all very similar to each other. However, while antiterminators such as SacT and SacY from B. subtilis have a relaxed specificity, i.e. they bind RAT sequences of the $b g l$ type in addition to their cognate targets, binding of the B. subtilis LicT and E. coli BglG antiterminators is restricted to their cognate bgl RAT sequences (Manival et al., 1997; Schnetz et al., 1996). The structures of the RNA-binding domains of SacY and LicT were determined and revealed a novel fold for RNA-binding proteins (Declerck et al., 1999; Manival et al., 1997; van Tilbeurgh et al., 1997). Until the 
recent determination of the solution structure of the LicT-RNA complex, the question of how a symmetric dimeric protein would recognize an apparently asymmetric RNA has remained enigmatic. It now turned out that the $b g l P$ RAT sequence adopts a quasi-symmetric structure which allows binding of the protein dimer (Yang et al., 2002). In contrast, the RAT sequence recognized by GlcT is only distantly related to the $b g l$ - and sac-type RAT sequences and no cross-talk was observed (Langbein et al., 1999). Homologs of GlcT and the respective RAT sequence were identified in Staphylococcus carnosus (Knezevic et al., 2000).

In this work, we studied the interaction of the RNA-binding domain of GlcT with its cognate RAT target. Several mutations in the RAT sequence were found to affect the antitermination efficiency in vivo. Mutations that restored expression of the ptsGHI operon in the presence of a non-functional RAT all destroyed the transcriptional terminator in the ptsGHI leader mRNA. The sites of interaction between the RNA-binding domain of GlcT and its RAT were studied by in vitro footprinting and mutagenesis. As predicted by Yang et al. (Yang et al., 2002), our results suggest that the RAT sequence may form a stem-loop structure with a nearly perfect symmetry, which is the recognition site for GlcT. Moreover, we identified a structural specificity determinant which decides the interaction partner.

\section{Materials and Methods}

\section{Bacterial strains and growth conditions}

The B. subtilis strains used in this study are shown in Table 6. Strains used in the random and site-directed mutagenesis studies are listed in Tables 7 and 8. All B. subtilis strains are derivatives of the wild type strain 168. Escherichia coli DH5a (Sambrook et al., 1989) was used for cloning experiments and for expression of recombinant proteins.

Bacillus subtilis was grown in SP medium or in CSE minimal medium (Faires et al., 1999). The media were supplemented with auxotrophic requirements (at $50 \mathrm{mg} / \mathrm{l}$ ) and carbon sources and inducers as indicated. Escherichia coli was grown in LB medium and transformants were selected on plates containing ampicillin $(100 \mu \mathrm{g} / \mathrm{ml})$. LB and SP plates were prepared by the addition of $17 \mathrm{~g} / 1$ Bacto agar (Difco) to LB or SP medium, respectively. 
Table 6. B. subtilis strains used in this study

\begin{tabular}{|c|c|c|}
\hline Strain & Genotype & Source $^{\mathrm{a}}$ \\
\hline 168 & $\operatorname{trp} C 2$ & Laboratory collection \\
\hline BGW10 & trpC2 lys-3 $\Delta$ licTS::erm & $\begin{array}{l}\text { Krüger and Hecker, } \\
1995\end{array}$ \\
\hline GM1010 & 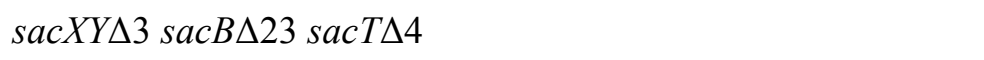 & Le Coq et al., 1995 \\
\hline QB5448 & $\operatorname{trp} C 2$ amyE::( $\Delta L A$ pts $G^{\prime}-{ }^{\prime} l a c Z$ aphA3) & Stülke et al., 1997 \\
\hline GP109 & $\operatorname{trp} C 2 \Delta$ glcT8 amyE::( $\triangle \mathrm{LA}$ ptsG'-'lacZ aphA3) & $\begin{array}{l}\text { Bachem and Stülke, } \\
1998\end{array}$ \\
\hline GP150 & $\operatorname{trpC2} \Delta g l c T 8$ amyE::('lacZ cat) & Langbein et al., 1999 \\
\hline GP151 & $\operatorname{trp} C 2$ amyE::( $\left(\mathrm{LA}_{\mathrm{C} 75 \mathrm{G} \mathrm{C} 76 \mathrm{G}} p t s G^{\prime}-' l a c Z\right.$ aphA3) & Langbein et al., 1999 \\
\hline GP169 & 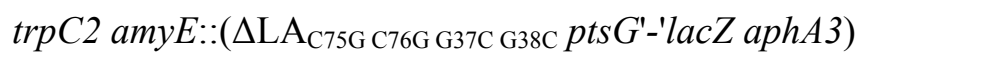 & pGP332 $\rightarrow 168$ \\
\hline GP172 & $\operatorname{trpC} 2$ amyE::( $\left(\mathrm{CA}_{\mathrm{G} 11 \mathrm{~A} \mathrm{G} 26 \mathrm{~A}} p t s G^{\prime}-' a p h A 3-' l a c Z\right.$ cat $)$ & $\mathrm{pGP} 339 \rightarrow 168$ \\
\hline GP173 & 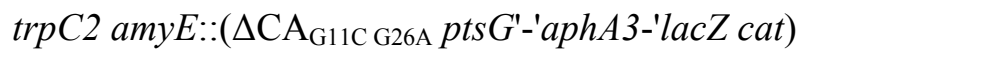 & $\mathrm{pGP} 340 \rightarrow 168$ \\
\hline GP174 & $\operatorname{trpC} 2 \operatorname{amyE}::(\triangle \mathrm{CA}$ ptsG'-'aphA3-'lacZ cat $)$ & pGP342 $\rightarrow 168$ \\
\hline GP385 & $\operatorname{trp} C 2$ amyE::( $\left(\mathrm{LA}_{\mathrm{A} \text {-ins }} p t s G^{\prime}-' l a c Z\right.$ aphA3) & pGP549 $\rightarrow 168$ \\
\hline GP386 & $\operatorname{trp} C 2$ amyE::( $\triangle \mathrm{LA}_{\mathrm{A} \text {-ins }} p t s G^{\prime}-{ }^{\prime} l a c Z$ aphA3) $\Delta g l c T 8$ & pGP549 $\rightarrow$ GP150 \\
\hline GP387 & amyE::( $(\mathrm{LA}$ ptsG'-'lacZ aphA3) sacXY $\triangle 3$ sacB $\Delta 23$ sacT $\Delta 4$ & pGP66 $\rightarrow$ GM1010 \\
\hline GP388 & 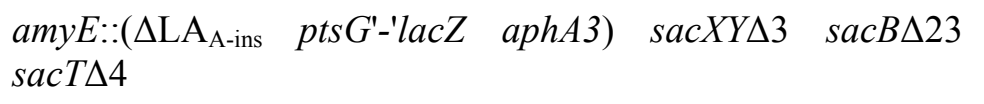 & pGP549 $\rightarrow$ GM1010 \\
\hline GP389 & 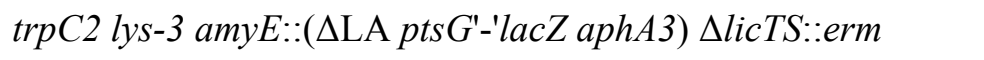 & pGP66 $\rightarrow$ BGW10 \\
\hline GP390 & 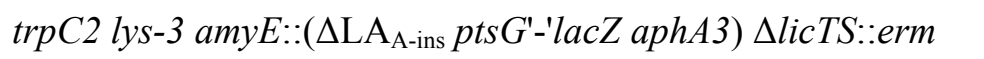 & pGP549 $\rightarrow$ BGW10 \\
\hline
\end{tabular}

${ }^{a}$ Arrows indicate construction by transformation.

\section{DNA manipulation}

Transformation of E. coli and plasmid DNA extraction were performed using standard procedures (Sambrook et al., 1989). Restriction enzymes, T4 DNA ligase and DNA polymerases were used as recommended by the manufacturers. DNA fragments were purified from agarose gels using a Nucleospin extract kit (Macherey and Nagel). Pfu DNA polymerase was used for PCR, as recommended by the manufacturer. The combined chain reaction was performed with $P f u$ DNA polymerase and thermostable DNA ligase (Ampligase ${ }^{\circledR}$; Epicentre, Madison, WI). DNA sequences were determined using the dideoxy chain termination method (Sambrook et al., 1989). Chromosomal DNA of B. subtilis was isolated as described (Kunst and Rapoport, 1995). 


\section{Site-directed mutagenesis of the pts $G$ terminator and RAT sequences}

Translational fusions of mutant variants of the $p t s G$ regulatory region with the lac $Z$ gene were constructed using the vector pAC7 (Weinrauch et al., 1991) containing the kanamycin resistance gene aphA3. The plasmid harbors a lacZ gene without a promoter located between two fragments of the B. subtilis amyE gene. To study the effect of point mutations in the conditional terminator and the RAT sequence preceding the pts $G$ structural gene the following strategy was applied. A DNA fragment carrying the mutant form of the terminator or ptsG-RAT was constructed by site-directed mutagenesis using PCR-based approaches as outlined previously (Bachem et al., 1997; Bi and Stambrook, 1998). Plasmid pGP66 (Stülke et al., 1997) containing the $p t s G$ promoter region served as a template. Multiple mutations were inserted using plasmids containing one or two mutations as templates. The mutagenic primers and the resulting plasmids are available upon request. The oligonucleotides JS11 (Stülke et al., 1997) and IL5 (Langbein et al., 1999) were used as outer primers. The final PCR products were purified and cut at the BamHI and MfeI sites introduced by the PCR primers. To introduce the constructed lacZ fusions into the chromosome of B. subtilis, competent cells of the wild type strain 168 were transformed with plasmids carrying the respective mutations linearized with $S c a \mathrm{I}$.

\section{Transformation and characterization of phenotype}

Bacillus subtilis was transformed with plasmid DNA according to the two-step protocol described previously (Kunst and Rapoport, 1995). Transformants were selected on SP plates containing chloramphenicol $(5 \mu \mathrm{g} / \mathrm{ml})$ or kanamycin $(5 \mu \mathrm{g} / \mathrm{ml})$. In B. subtilis, amylase activity was detected after growth on SP medium supplemented with $10 \mathrm{~g} / 1$ hydrolyzed starch (Connaught). Starch degradation was detected by sublimating iodine onto the plates.

Quantitative studies of lacZ expression in B. subtilis in liquid medium were performed as follows. Cells were grown in CSE medium supplemented with the carbon sources indicated. Cells were harvested at $\mathrm{OD}_{600}=0.6-0.8$. Cell extracts were obtained by treatment with lysozyme and DNase. B-Galactosidase activity was determined as previously described using o-nitrophenyl-galactoside as substrate (Kunst and Rapoport, 1995). One unit is defined as the amount of enzyme which produces $1 \mathrm{nmol} o$-nitrophenol per min at $28^{\circ} \mathrm{C}$.

\section{Protein purification}

To purify the RNA-binding domain of GlcT as a native protein, plasmid pGP230 was constructed as follows. Plasmid pGP114 (Langbein et al., 1999) containing the DNA 
fragment corresponding to amino acids 1-60 of GlcT fused to an N-terminal hexahistidine sequence was linearized with BamHI. The oligonucleotides MM6 (5'-GATCTCTGGTTCCGCGTGGTTCCATGA) and MM7 (5'-GATCTCATGGAACC ACGCGGAACCAGA) carrying a DNA fragment encoding a thrombin cleavage site were hybridized at $80^{\circ} \mathrm{C}$ and ligated to the linearized plasmid pGP114. Positive candidates were verified by sequencing.

Escherichia coli DH5 $\alpha$ was used as host for overexpression of the recombinant proteins. Expression was induced by the addition of IPTG (final concentration $1 \mathrm{mM}$ ) to logarithmically growing cultures $\left(\mathrm{OD}_{600}=0.8\right)$. The crude extracts were passed over Ni-NTA Superflow (Qiagen), followed by elution with an imidazole gradient. The Bio-Rad dyebinding assay was used to determine protein concentration. Bovine serum albumin was used as the standard. Purified His::GlcT-RBD protein was concentrated using a centriprep concentrator unit (Millipore). The GlcT-RBD peptidic fragment (7498 Da) was generated by thrombin cleavage (Pharmacia) of the His::GlcT-RBD protein according to the supplier's instructions (Pharmacia). After thrombin cleavage the protein preparation was loaded onto a Superdex 75 prep grade HR16/60 column (Pharmacia) for size exclusion chromatography in $300 \mathrm{mM} \mathrm{NaCl}, 50 \mathrm{mM} \mathrm{Na} \mathrm{HPO}_{4}(\mathrm{pH} \mathrm{7.8)}$. The purity of the protein was determined on 15\% Tris-Tricine gels using Coomassie brilliant blue staining (Schägger and von Jagow, 1987).

\section{In vitro transcription}

To obtain a template for in vitro synthesis of the wild type RAT RNA, a 84 bp PCR product was generated using pGP66 (Stülke et al., 1997) as template and primers IL59 (5'-CCAAGTAATACGACTCACTATAGGACGTGTTACTGATTCG) and IL60 (5'-CAAG AATTGGGACAACTCTTCTTCTCCTTTTTTTTCCTCAATCACTCATGCC). To generate a template for in vitro transcription of mutant RAT RNAs, the primers OS25 (5'-CCAAGTAATACGACTCACTATAGGAATTCAGTTTATCCTTAT) and OS26 (5'-TTG AGGGAAAAAAACGGGAAGTTC) were used to amplify a $99 \mathrm{bp}$ PCR product. The presence of a T7 RNA polymerase recognition site in primers IL59 and OS25 (underlined) allowed the use of the PCR product as a template for in vitro transcription with T7 RNA polymerase (Roche Diagnostics). As a non-specific RNA, a 350 bp gapA transcript was prepared as described previously. The integrity of the RNA transcripts was analyzed by denaturating agarose gel electrophoresis (Ludwig et al., 2001). 


\section{Assay of interaction between GIcT-RBD and RAT RNA}

Binding of GlcT-RBD to RAT RNA was analyzed by gel retardation experiments. The RAT RNA (in water) was denatured by incubation at $90^{\circ} \mathrm{C}$ for 2 min and renatured by dilution 1:1 with ice-cold water and subsequent incubation on ice. If required, non-specific RNA was renatured separately and mixed with the RAT RNA prior to protein addition. Purified GlcTRBD was added to the RAT RNA and the samples were incubated for $10 \mathrm{~min}$ at room temperature. After this incubation, glycerol was added to a final concentration of $10 \%(\mathrm{wt} / \mathrm{vol})$. The samples were then analyzed on $12 \%$ Tris-acetate polyacrylamide gels.

\section{RNase T1 footprinting}

The method used for RNase T1 digestion of the RAT RNA was as follows. Radiolabeled RNA was denatured by heating to $90{ }^{\circ} \mathrm{C}$ and was subsequently allowed to cool down at room temperature. Diluted GlcT-RBD (7 pmol in $1 \mu \mathrm{l})$ was added to 5'-radiolabeled RAT RNA (140 000 c.p.m., gel purified) in the presence of $20 \mathrm{mM} \mathrm{MgCl}_{2}$ and $300 \mathrm{mM} \mathrm{NaCl}$ (final concentration). The reaction was incubated at $37^{\circ} \mathrm{C}$ for $10 \mathrm{~min}$, after which $20 \mu \mathrm{g}$ yeast tRNA (Sigma) and appropriate diluted RNase T1 (1 or $2 \mathrm{U}$ ) was added, followed by 15 min incubation at $37^{\circ} \mathrm{C}$. The reaction was stopped by adding another $20 \mu \mathrm{g}$ tRNA. The control reactions were performed in the absence of protein or RNase T1 under corresponding conditions. The RAT RNA was extracted with acid phenol/chloroform, precipitated with ethanol, resuspended and analyzed by electrophoresis.

\section{Screening system to isolate random terminator mutants}

Plasmids containing the mutated $p t s G$-RAT and a promoterless kanamycin resistance cassette were obtained as follows. The mutated $p t s G$-RAT alleles were amplified via PCR using the plasmids pGP329 and pGP330 as template DNA (obtained by site-directed mutagenesis of the RAT sequence; see above) and the oligonucleotides IL5 (Langbein et al., 1999) and JS11 (Stülke et al., 1997). The PCR products were digested with $M f e \mathrm{I}$ and BamHI and the $0.5 \mathrm{~kb}$ fragment was cloned into pAC6 (Stülke et al., 1997) cut with the same enzymes. The resulting plasmids were designated pGP337 and pGP338. In a second step a promoterless aphA3 kanamycin resistance cassette (Trieu-Cuot and Courvalin, 1983) was amplified using the primers IL46 (5'-CGGGATCCTAATGTTAGAAAAGAGGAAGGAAATAA) and IL48 (5'-CGGGATCCCTACTAAAACAATTCATCCAGTAA) and pAC7 as template DNA. The DNA fragment was cut with BamHI and cloned into the target vectors pGP337 and pGP338 linearized with the same enzyme. The resulting plasmids were designated pGP339 and 
pGP340, respectively. Moreover, by the same strategy we constructed plasmid pGP342 harboring a $p t s G-a p h A 3$ lacZ fusion under the control of the wild type regulatory elements. These plasmids were used to introduce a ptsG-aphA3 lacZ fusion into the chromosome of B. subtilis 168 (see Table 6).

Liquid cultures of GP172 and GP173 containing the artificial ptsG-aphA3 lacZ operon were grown overnight in the absence of kanamycin. Aliquots of $100 \mu 1$ of independently grown cultures were plated onto SP plates containing kanamycin at a final concentration of 30 and $60 \mu \mathrm{g} / \mathrm{ml}$, respectively. The plates were incubated for 2 days at $28{ }^{\circ} \mathrm{C}$ to select for colonies able to grow on kanamycin and express the ptsG-aphA3 lacZ fusion. To verify the localization of the selected mutations in the control region of the artificial operon, B. subtilis 168 was transformed with chromosomal DNA of the mutant strains and transformants were selected for chloramphenicol resistance. Transformants resistant to both chloramphenicol and kanamycin contained the mutation in close proximity to the cat resistance gene, which is located downstream of the lacZ gene. The $p t s G$ promoter regions of these strains were analyzed by sequencing.

\section{Results}

\section{Mutational analysis of the ptsG terminator sequence}

In a previous work, we demonstrated that a mutation disrupting the presumed stem structure of the transcriptional terminator in the $p t s G$ leader region resulted in constitutive expression of ptsG (Langbein et al., 1999). To ensure that it is the structure of the terminator rather than its sequence we constructed a strain, GP169, carrying a compensatory mutation in the terminator region. In this strain, the potential stem structure of the terminator is restored without correcting the sequence. The pts $G$ promoter region containing this mutation was inserted into the chromosome of B. subtilis in front of a promoterless lac $Z$ gene. The synthesis of B-galactosidase after growth of the strains in CSE minimal medium in the absence and presence of glucose was monitored to assay transcription driven by the wild type and mutant $p t s G$ promoter regions (Table 7). While transcription was inducible by glucose in the wild type strain QB5448, constitutive expression was observed in the terminator mutant GP151. 
Table 7. Effect of mutations in the terminator on expression of a $p t s G-l a c Z$ fusion

\begin{tabular}{llll}
\hline Strain & Terminator & \multicolumn{2}{l}{ B-Galactosidase activity (U/mg protein) } \\
\hline & & - Glucose & + Glucose \\
QB5448 & Wild type & 19 & 294 \\
GP151 & C75G C76G & 779 & 360 \\
GP169 & G37C G38C C75G C76G & 18 & 204 \\
GP174 & Wild type & 5 & 450 \\
GP172 & 'Wild type' (G11A G26A) & 3 & 12 \\
GP186 & GP172 $\Delta 4$ & 824 & 584 \\
GP187 & GP172 G72U & 49 & 120 \\
GP188 & GP172 $\Delta 3$ & 841 & 651 \\
GP189 & GP172 G28U & 497 & 445 \\
GP190 & GP172 C75A & 133 & 177 \\
GP191 & GP172 G47U & 405 & 368 \\
GP173 & 'Wild type' (G11C G26A) & 7 & 11 \\
GP179 & GP173 $\Delta 1$ & 1635 & 1725 \\
GP181 & GP173 G38A & 71 & 95 \\
GP182 & GP173 G47U & 475 & 337 \\
GP183 & GP173 $\Delta 2$ & 796 & 510 \\
GP184 & GP173 C75U & 98 & 113 \\
GP185 & GP173 G90A & 170 & 150 \\
\hline
\end{tabular}

${ }^{\text {a }}$ Representative values of lacZ expression. All measurements were performed at least twice.

These results are in good agreement with previous findings (Langbein et al., 1999). The newly constructed compensatory mutation restored the inducibility of $p t s G$ expression and therefore the functionality of the transcriptional terminator. Thus, the structure of the terminator seems to be important rather than its actual sequence. The restoration of the terminator structure results in transcription termination in the absence of the inducer, glucose. To provide further evidence for the functional role of the terminator, we developed a screening and selection system that allowed us to select for spontaneous mutations resulting in constitutive ptsG expression. Briefly, an artificial aphA3 lacZ operon was created and placed under control of the pts $G$ expression signal. The two strains used here (GP172 and GP173) carried mutations of the RAT sequence that prevented their recognition by GlcT. Mutant strains allowing expression of the artificial operon were selected as decribed in Materials and Methods. 


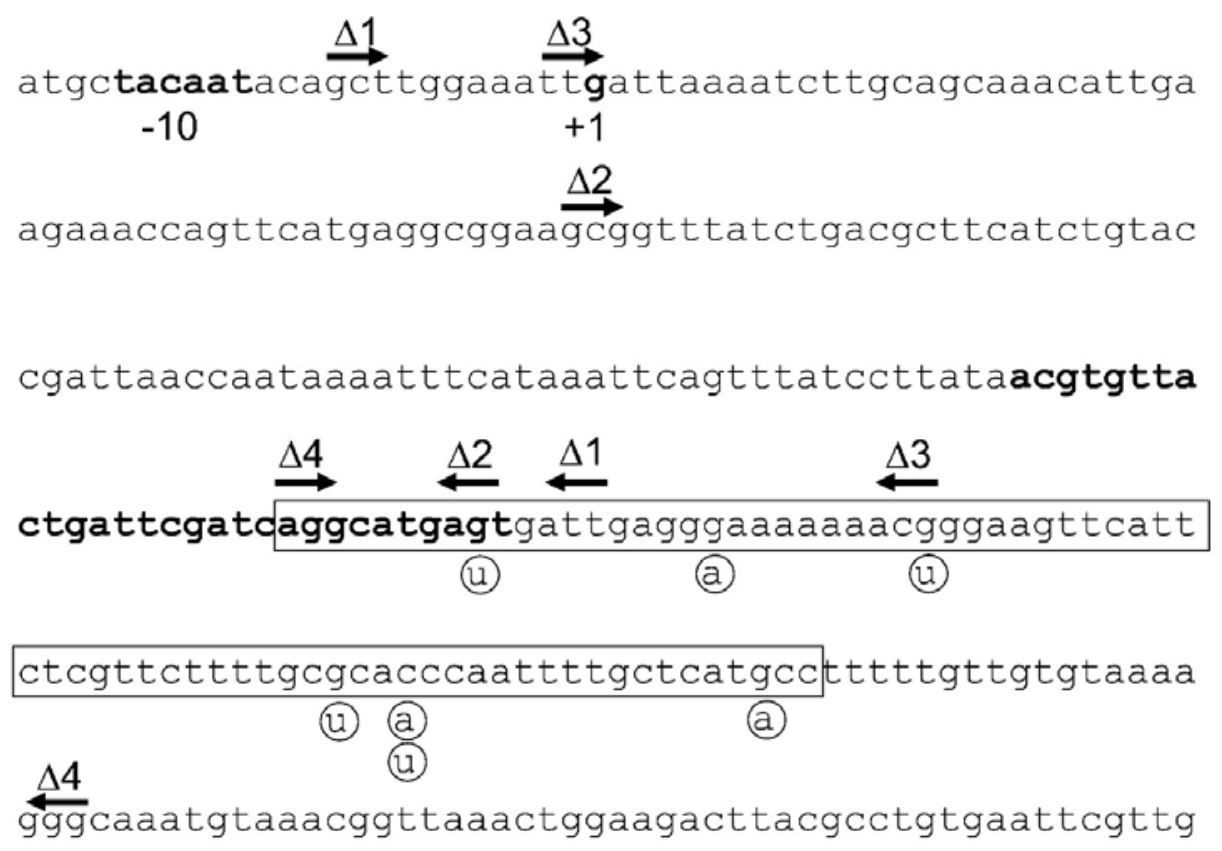

Figure 4.2. Sequence of the ptsG promoter region. The RAT sequence is shown in bold and the terminator is boxed. The -10 promoter region and the transcription start site are shown in bold. The identified point mutations are shown in circles below the original nucleotides. Arrows with numbers indicate the deleted regions found in the sequenced mutants. The strain designations, the 'numbering' of the positions and the effects of the mutations are given in Table 7.

Several mutants exhibiting both resistance to kanamycin and constitutive expression of the lacZ gene (as monitored by the blue color of colonies on X-Gal plates) were obtained and their ptsG promoter regions upstream of the aphA3 lacZ operon were sequenced. The mutations all affected the transcriptional terminator in the pts $G$ leader region. A total of 12 different spontaneous mutations, six derived from each strain, were identified. Among these mutations were four deletions encompassing the terminator region and eight point mutations. One of these point mutations $\left(\mathrm{G}_{47} \mathrm{~T}\right)$ was obtained with both original strains, whereas two different mutants obtained with either GP172 and GP173 affected the same position of the terminator $\left(\mathrm{C}_{75} \mathrm{~A}\right.$ and $\mathrm{C}_{75} \mathrm{~T}$, respectively). A summary of the sequence information concerning the mutations is shown in Figure 4.3. The effect of the mutations was quantified by assaying the $\beta$-galactosidase activity driven by the mutant pts $G$ control elements. As expected, inducible expression was observed in the wild type strain GP174, while no expression of the lacZ gene was detected in the mutant strains GP172 and GP173 that were used to select for terminator mutants. The mutations of the terminator all resulted in constitutive expression of the artificial aphA3 lacZ operon. However, the absolute level of expression was different for 
the individual mutants. The deletions and the mutation of $\mathrm{G}_{47}$ resulted in high constitutive expression (400-1700 U ß-galactosidase/mg protein; see Table 7). A point mutation at position $\mathrm{C}_{75}$ or $\mathrm{G}_{90}$ resulted in intermediate expression (100-200 U ß-galactosidase/mg protein), while the mutations of $\mathrm{G}_{38}$ and $\mathrm{G}_{72}$ resulted in weak constitutive expression $(<100 \mathrm{U}$ ß-galactosidase/mg protein; see Table 7).

Taken together, our findings indicate that the terminator is the only negatively acting regulatory element in the $p t s G$ promoter region. Moreover, it is the secondary structure rather than the actual sequence of the terminator which is important for the control of ptsG expression.

\section{Binding of the RNA-binding domain of GIcT to the RAT sequence}

Previous genetic and biochemical evidence suggested direct binding of GlcT to the RAT sequence that overlaps the terminator in the pts $G$ leader mRNA. To substantiate these observations and to study the molecular details of protein-RNA interaction, we first purified the N-terminal domain of GlcT devoid of a His tag. In a previous study, binding of His-tagged GlcT to the RAT RNA was demonstrated (Langbein et al., 1999). However, subsequent gel retardation experiments suggested that the affinity tag might cause some non-specific RNAbinding (our unpublished results). We therefore constructed a system that allowed removal of the His tag after initial purification of the protein.

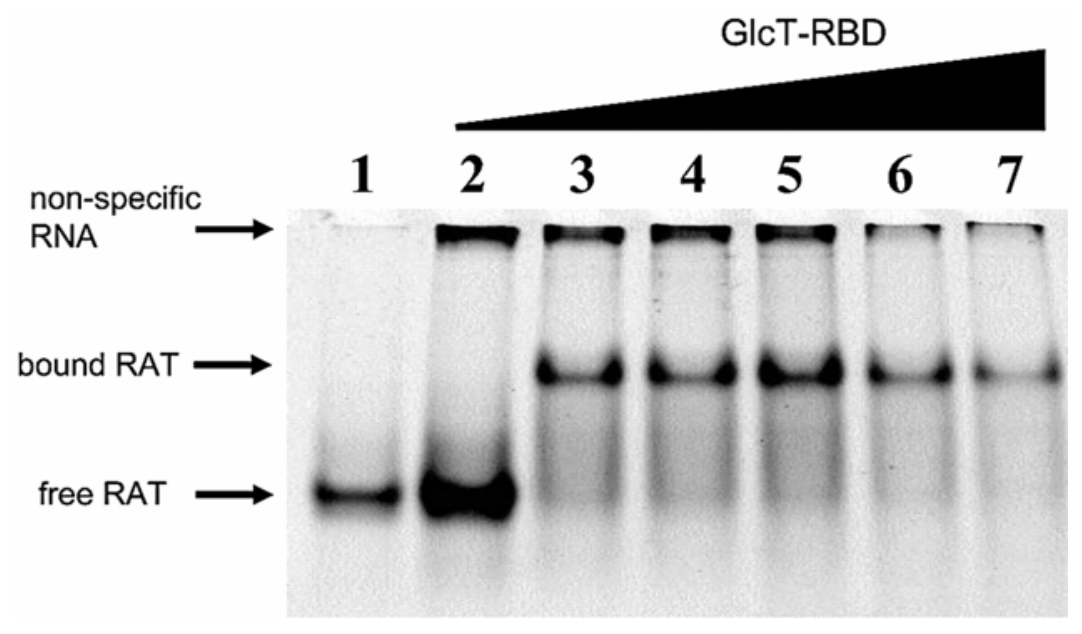

Figure 4.3. Electrophoretic mobility band shift analysis of the interaction between the RAT and the RBD. Lane 1 shows $125 \mathrm{pmol}$ of the free RAT RNA. In lanes 2-7, 250 pmol RAT RNA and 30 pmol non-specific RNA were used. Increasing concentrations of RBD-GlcT were added to the RNA prior to electrophoresis. Aliquots of 250, 500, 750, 1000 and 1500 pmol RBD-GlcT were used. The arrows indicate the different RNA species. 
The purified protein was efficiently cleaved at the introduced thrombin cleavage site. The resulting protein was purified to apparent homogeneity by size exclusion chromatography as described in Materials and Methods.

The ability of the in vitro generated RNA-binding domain of GlcT to bind its RNA target was tested by electrophoretic mobility shift analysis. As shown in Figure 4.4, addition of the RNA-binding domain to the RAT RNA resulted in a shift of the apparent size of the RAT fragment. At a high concentration of the RNA-binding domain (3-fold molar excess), the RAT RNA was completely shifted (see Fig. 4.3, lane 7). The shifted band contained a complex of both the RNA and the RNA-binding domain, as suggested by the differential detection of both components with ethidium bromide (detection of RNA) and Coomassie brilliant blue (detection of protein) (data not shown).

\section{Structural analysis of the GIcT-RAT interaction by RNase T1 protection footprint analysis}

Specificity determinants have already been studied for the RAT sequences of the $b g l$ and the $s a c$ type and the structure of the complex between the bglP RAT and the LicT antiterminator was determined (Aymerich and Steinmetz, 1992; Yang et al., 2002). However, there is no cross-talk between the antiterminators of the $\mathrm{Bgl}$ and Sac types and the pts $G$ RAT sequence and GlcT is not capable of causing antitermination at $b g l$ or $s a c$ RAT sequences (Langbein et $a l ., 1999)$. Thus GlcT and the ptsG RAT may be the most distant members of this large family of protein-dependent riboswitches. This prompted us to initiate an analysis of the molecular details of the interaction between the RNA-binding domain of GlcT and the ptsG RAT sequence by RNase $\mathrm{T} 1$ footprint analysis.

RNase T1 specifically cleaves adjacent to G residues in single-stranded regions of the RNA. Binding of the G residue to a protein or formation of a double-stranded RNA structure protects the RNA from degradation by RNase T1. As expected, incubation of denatured RAT RNA with RNase T1 resulted in RNA cleavage at each of the G residues in the RAT sequence (see Fig. 4.4, lane 2). Similarly, all G residues are available for cleavage by RNase T1 in the native form of the RAT RNA (see Fig. 4.4, lanes 3 and 5). Thus, the RAT does not seem to adopt a secondary structure in the absence of GlcT. In the presence of the RNA-binding domain of GlcT several G residues were protected from nucleolytic degradation, with only one $\mathrm{G}$ residue remaining accessible. 
A.

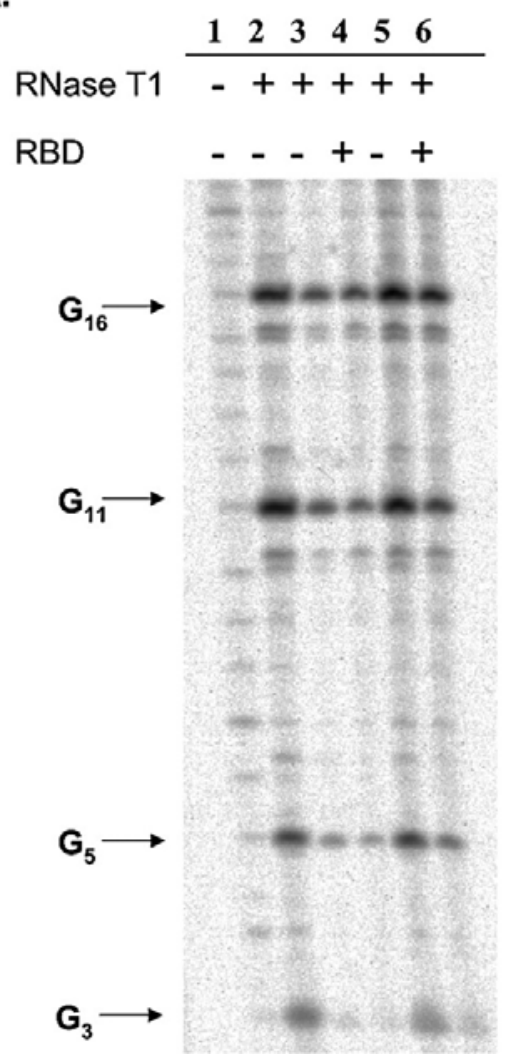

B.

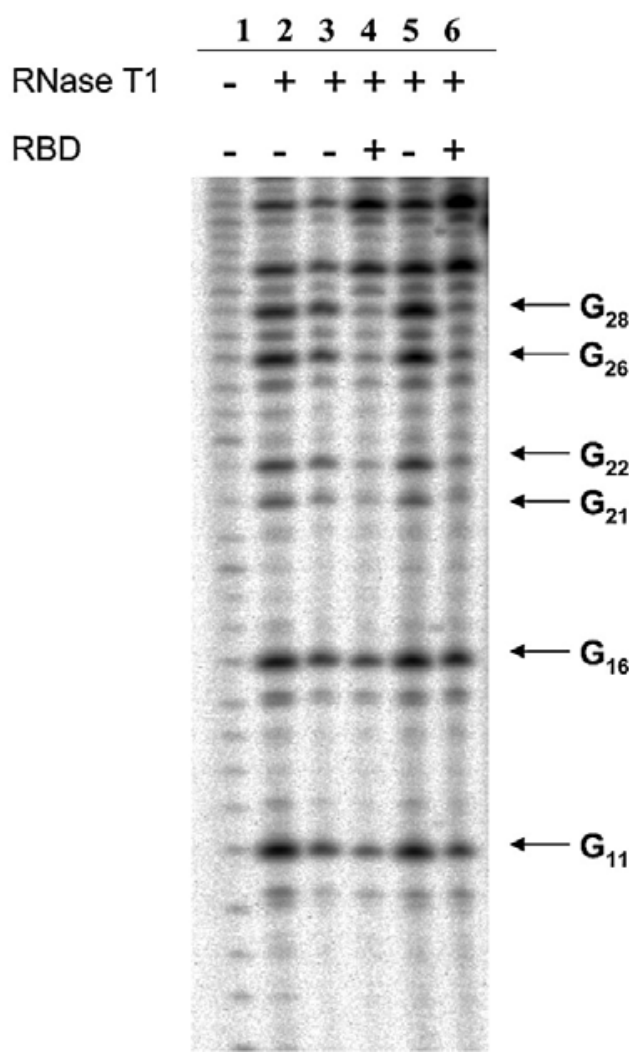

Figure 4.4. RNase T1 footprint of the ptsG RAT region. Two different polyacrylamide concentrations were used to resolve the complete region: (A) 26\%; (B) 20\%. Weak hydrolysis of the RNA in the absence of the RNase and the antiterminator protein shows every nucleotide of the RAT (lane 1). High concentrations of RNase T1 under denaturing conditions leads to cleavage at every guanine nucleotide (lane 2). In the other assays, the RAT RNA and the RBD were used as indicated. In lanes 5 and 6 , twice as much radiolabeled RNA was used as compared to lanes 3 and 4 . Arrows indicate the position of the guanine nucleotides in the RAT.

This residue, $\mathrm{G}_{16}$, is probably located in the top loop of the RAT secondary structure and thus part of a single-stranded region in the absence and in the presence of GlcT. Among the residues protected by GlcT, $\mathrm{G}_{22}, \mathrm{G}_{26}$ and $\mathrm{G}_{28}$ were most strongly protected. According to the secondary structure model of the RAT RNA (see Figs. 4.1 and 4.7), $\mathrm{G}_{22}$ and $\mathrm{G}_{26}$ are located in loop regions of the RAT RNA and may thus be protected by interaction with GlcT. $\mathrm{G}_{3}, \mathrm{G}_{11}$ and $\mathrm{G}_{21}$ were weakly protected in the presence of GlcT. This may reflect their location in a double-stranded region rather than interaction with GlcT. Indeed, $\mathrm{G}_{11}$ and $\mathrm{G}_{21}$ are both thought to be located in the third double-stranded region of the RAT structure. Thus, the footprinting data indicate that the RAT RNA exists in the single-stranded form in the absence of GlcT and adopts the double-stranded conformation upon GlcT addition. Moreover, GlcT seems to interact with residues in the two loops of the RAT structure. 


\section{Probing of the RAT structure and of protein-RNA interactions by site-directed mutagenesis}

Since only the guanine residues can be probed by RNase T1 footprinting, we attempted to study the role of the individual bases of the RAT sequence in more detail by site-directed mutagenesis. Mutations of the RAT sequence were introduced into the $p t s G$ promoter region, located in front of a promoterless lacZ gene. If the mutations in the RAT sequence were expected to affect the structural integrity of the overlapping terminator, we introduced compensatory mutations in the terminator to restore the presumed wild type secondary structure (see Fig. 4.1).

To assay the effects of the different mutations, the strains were grown in CSE minimal medium in the absence and presence of glucose and the $\beta$-galactosidase activities were determined as an indicator of termination/antitermination activity. The results are summarized in Table 8. As observed previously, a strong induction of the $p t s G-l a c Z$ fusion was detected for the wild type strain QB5448.

Mutations preventing formation of the first double-stranded region of the RAT RNA resulted in loss of induction (GP367, $\left.\mathrm{C}_{2} \mathrm{G} ; \mathrm{GP} 368, \mathrm{G}_{28} \mathrm{C}\right)$. Interestingly, a strain with an inverse arrangement of the $\mathrm{C}$ and $\mathrm{G}$ residues (and an additional compensatory mutation in the terminator, GP366; see Table 8) resulted in constitutive expression rather than in restoration of inducibility. Constitutive expression is a strong indication of inactivity of the transcriptional terminator. Thus, the overall structure of the RAT/terminator region may have changed in such a way that transcription termination no longer occurs in the triple mutant GP366. To address this hypothesis, we assayed the activity of the $p t s G$ control region present in GP366 in a $g l c T$ background. While no expression of the wild type $p t s G-l a c Z$ fusion was observed in the $g l c T$ mutant GP109, constitutive expression was detected for the $\mathrm{C}_{2} \mathrm{G}_{28} \mathrm{C}$ $\mathrm{C}_{85} \mathrm{G}$ triple mutant (data not shown). This result confirms that antitermination is not required to express $\beta$-galactosidase in this construct. To test whether GlcT binds the RAT sequence with the inverse C:G base pair, we performed a gel mobility shift assay. As shown in Figure 4.6, this fragment was as efficiently bound by GlcT as the wild type RAT fragment. In contrast, a fragment containing only the $\mathrm{C}_{2} \mathrm{G}$ mutation that interferes with formation of the lowest part of the stem was barely shifted by GlcT. Thus, formation of this stem is important for RNA-binding by GlcT, whereas the actual sequence does not seem to be critical. 
Table 8. Effect of mutations in the RAT sequence on expression of a $p t s G-l a c Z$ fusion

\begin{tabular}{|c|c|c|c|c|}
\hline \multirow[t]{2}{*}{ Strain } & \multirow[t]{2}{*}{ RAT } & \multirow[t]{2}{*}{ Compensatory mutation (terminator) } & \multicolumn{2}{|c|}{$\begin{array}{l}\text { B-Galactosidase } \\
(\mathrm{U} / \mathrm{mg} \text { protein })^{\mathrm{a}}\end{array}$} \\
\hline & & & - Glucose & + Glucose \\
\hline QB5448 & Wild type & & 11 & 610 \\
\hline GP367 & $\mathrm{C} 2 \mathrm{G}$ & & 17 & 7 \\
\hline GP368 & G28C & $\mathrm{C} 85 \mathrm{G}$ & 29 & 18 \\
\hline GP366 & $\mathrm{C} 2 \mathrm{G} \mathrm{G} 28 \mathrm{C}$ & $\mathrm{C} 85 \mathrm{G}$ & 360 & 302 \\
\hline GP375 & $\mathrm{G} 3 \mathrm{C}$ & & 14 & 3 \\
\hline GP376 & G3A & & 20 & 4 \\
\hline GP380 & $\mathrm{A} 27 \mathrm{U}$ & U86A & 9 & 7 \\
\hline GP381 & $\mathrm{A} 27 \mathrm{G}$ & $\mathrm{U} 86 \mathrm{C}$ & 686 & 1053 \\
\hline GP370 & G26U & G87A & 64 & 1038 \\
\hline GP379 & $\mathrm{U} 25 \mathrm{G}$ & $\mathrm{A} 88 \mathrm{C}$ & 8 & 12 \\
\hline GP369 & G5C C23G & G90C & 14 & 277 \\
\hline GP377 & U6G & & 7 & 3 \\
\hline GP378 & U6A & & 26 & 4 \\
\hline GP371 & $\mathrm{U} 7 \mathrm{G}$ & & 15 & 225 \\
\hline GP175 & U7C & & 9 & 580 \\
\hline GP176 & A $8 \mathrm{G}$ & & 8 & 58 \\
\hline GP177 & U7C A8G & & 33 & 60 \\
\hline GP373 & $\mathrm{G} 22 \mathrm{C}$ & C91G & 12 & 1 \\
\hline GP374 & G22U & C91A & 7 & 2 \\
\hline GP178 & $\mathrm{C} 9 \mathrm{U}$ & & 5 & 21 \\
\hline GP372 & C9G G21C & C92G & 376 & 418 \\
\hline GP160 & G11A & & 6 & 82 \\
\hline GP166 & A12U U13A & & 5 & 353 \\
\hline GP156 & U14A & & 12 & 367 \\
\hline GP158 & $\mathrm{C} 15 \mathrm{U}$ & & 7 & 492 \\
\hline GP159 & $\mathrm{C} 15 \mathrm{~A}$ & & 7 & 562 \\
\hline
\end{tabular}

\footnotetext{
${ }^{\mathrm{a}}$ Representative values of lacZ expression. All measurements were performed at least twice.
} 

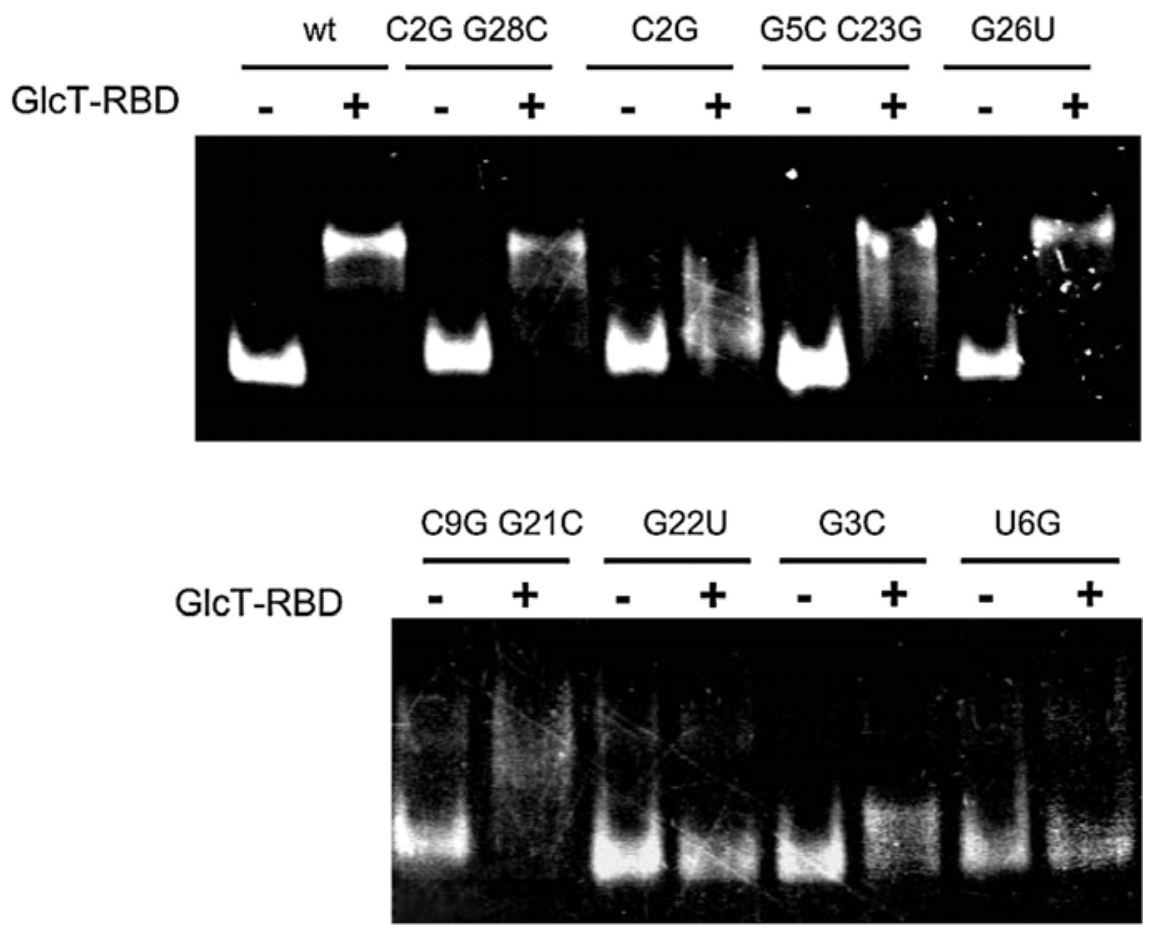

Figure 4.5. Electrophoretic mobility band shift analysis of the interaction between different mutant RAT species and the RBD.

Mutations affecting loop 1 of the RAT had different effects: two different mutations affecting the $\mathrm{G}_{3}$ residue resulted in complete loss of induction. This finding is in good agreement with the observed role of this residue in the interaction with GlcT as inferred from the RNase T1 protection experiment. Moreover, a RAT sequence containing the $\mathrm{G}_{3} \mathrm{C}$ mutation is not bound by GlcT in vitro (see Fig. 4.5). However, both mutations $\mathrm{G}_{3} \mathrm{C}$ and $\mathrm{G}_{3} \mathrm{~A}$ may alternatively affect the structure of the RAT RNA, as both introduced bases would find pairing partners on the opposing side of the RAT $\left(\mathrm{U}_{25}\right.$ or $\left.\mathrm{G}_{26}\right)$. Similarly, mutations affecting the residues $\mathrm{U}_{25}$ and $\mathrm{A}_{27}$ resulted in loss of antitermination. These mutations were not expected to alter the secondary structure of the RAT. However, the compensatory mutation for one of the mutations $\left(\mathrm{U}_{86} \mathrm{C}\right.$ in $\left.\mathrm{GP} 381\right)$ results in loss of terminator activity and thus in constitutive expression of $\beta$-galactosidase. Finally, the mutation $\mathrm{G}_{26} \mathrm{U}$ present in $\mathrm{GP} 370$ resulted in increased expression of the $p t s G-l a c Z$ fusion, both in the absence and presence of glucose. Here, the terminator may be leaky due to the compensatory mutation (A:U instead of $\mathrm{G}: \mathrm{C}$ base pair) and allow some basal expression even in the absence of glucose. Alternatively, the perfectly symmetrical structure of the RAT generated by the $\mathrm{G}_{26} \mathrm{U}$ mutation might result in improved binding of GlcT, including some weak antitermination in the absence of glucose. Indeed, the mutated RAT seems to be an efficient target for GlcT, as judged from the clear 
induction of $\beta$-galactosidase in this strain. In addition, the RAT RNA containing this mutation was efficiently bound by GlcT in vitro (see Fig. 4.5). Moreover, expression of this ptsG-lacZ fusion was completely lost in a glcT mutant strain (see Table 9). Taken together, our data indicate that loop 1 is of great importance for the proper interaction of the RAT RNA with GlcT.

One mutant affected in the second short double-stranded region of the RAT was studied. In this strain, GP369, the predicted G:C base pair was inverted to be C:G, and efficient induction of $\beta$-galactosidase activity was detected. Additionally, the RAT RNA carrying the inversion interacted with the RNA-binding domain of GlcT in vitro (see Fig. 4.5). Thus, the structure rather than the actual nucleotide at this position is important for GlcT binding and antitermination. This is in good agreement with the observed weak protection of $\mathrm{G}_{5}$ from RNase $\mathrm{T} 1$ digestion in the presence of GlcT.

Several mutations in loop 2 resulted in loss of GlcT binding to the RAT and thus in loss of antitermination, namely those affecting $\mathrm{U}_{6}$ and $\mathrm{G}_{22}$. Results obtained with the ptsG-lacZ fusion (Table 8) and the in vitro RAT-GlcT binding assay (see Fig. 4.5) are in perfect agreement for these mutations. In contrast, replacement of $U_{7}$ and/or $A_{8}$ showed no or only slight effects. The importance of $\mathrm{G}_{22}$ for efficient transcriptional antitermination is consistent with the strong RNase T1 protection obtained for this base in the presence of GlcT. However, similarly to loop 1, not all bases seem to play an important role in GlcT recognition.

As we approached the top of the proposed RAT structure, the mutations had lesser effects. The first base pair of the third double-stranded region is important for antitermination, as judged from lack of expression of the $p t s G-l a c Z$ fusion in the corresponding mutant strain GP178. An inversion of this base pair along with the required compensatory mutation in the terminator caused constitutive expression (GP372), thus we are unable to decide whether this inversion would affect antitermination. The constitutive expression of $\beta$-galactosidase in this strain was not abolished by deletion of the $g l c T$ gene (data not shown), indicating that the mutation inactivated the terminator. An in vitro assay of GlcT binding to the mutant RAT suggested that an interaction occurred, albeit the complex seemed to be less stable (see Fig. 4.5). A mutation of $\mathrm{G}_{11}$ had a mild effect whereas the $\mathrm{A}_{12} \mathrm{U} \mathrm{U}_{13} \mathrm{~A}$ double mutation present in GP166 did not influence the antitermination activity of GlcT. Similarly, three mutations in the top loop of the RAT did not affect induction by glucose and, therefore, binding of GlcT. These results indicate that the upper part of the RAT may only be required to obtain the correct RAT structure and is not involved in the interaction with GlcT. 
Table 9. Effect of mutations in the $p t s G$ RAT sequence on induction of a $p t s G-l a c Z$ fusion

\begin{tabular}{|c|c|c|c|c|c|c|}
\hline \multirow[t]{2}{*}{ Strain } & \multirow{2}{*}{$\begin{array}{l}\text { Relevant } \\
\text { genotype }\end{array}$} & \multicolumn{5}{|c|}{ ß-Galactosidase activity (U/mg protein) ${ }^{\mathrm{a}}$} \\
\hline & & CSE & CSE Glc & CSE Suc $(0.1 \%)$ & CSE Suc $(2 \%)$ & CSE Sal \\
\hline QB5448 & Wild type & 9 & 548 & 231 & 329 & 267 \\
\hline GP109 & $\Delta g l c T$ & 4 & 5 & 2 & 8 & 9 \\
\hline GP387 & $\Delta s a c T \Delta s a c Y$ & 10 & 327 & 85 & 166 & 335 \\
\hline GP389 & $\Delta l i c T$ & 12 & 412 & 222 & 422 & 312 \\
\hline GP385 & $\mathrm{RAT}-\mathrm{A}_{\mathrm{ins}}$ & 6 & 8 & 37 & 34 & 102 \\
\hline GP386 & $\mathrm{RAT}-\mathrm{A}_{\text {ins }} \Delta g l c T$ & 10 & 18 & 48 & 49 & 121 \\
\hline GP388 & $\begin{array}{l}\text { RAT-A } \mathrm{A}_{\text {ins }} \quad \Delta s a c T \\
\Delta s a c Y\end{array}$ & 6 & 11 & 5 & 6 & 110 \\
\hline GP390 & $\mathrm{RAT}^{-\mathrm{A}_{\text {ins }}} \Delta l i c T$ & 16 & 11 & 14 & 17 & 13 \\
\hline GP370 & $\mathrm{RAT}-\mathrm{G}_{26} \mathrm{U}$ & 38 & 790 & 622 & 740 & 665 \\
\hline GP392 & $\mathrm{RAT}-\mathrm{G}_{26} \mathrm{U} \Delta g l c T$ & 8 & 7 & 13 & 44 & 29 \\
\hline
\end{tabular}

${ }^{a}$ Representative values of lacZ expression. All measurements were performed at least twice.

\section{Converting the recognition specificity of the RAT sequence to LicT binding}

A comparison of RAT sequences of the glucose, sucrose, B-glucoside and lactose classes revealed that all but the glucose RAT sequences contain $3 \mathrm{bp}$ in the second double-stranded region whereas the pts $G$ RAT sequences of $B$. subtilis and $S$. carnosus contain only 2 bp (see Fig. 4.6). Moreover, loop 1 of the pts $G$ RAT is made up of four putatively non-paired bases whereas three non-paired bases form this loop in the other RAT sequences (Knezevic et al., 2000; Stülke et al., 1997; Yang et al., 2002) (see Fig. 4.6). Our mutagenesis studies suggested an important role of RAT structure in the loop regions for the interaction with GlcT. We therefore asked whether this unique arrangement of the $p t s G$ RAT is important for induction specificity by GlcT.

To address this question we constructed a $B$. subtilis strain with a pts $G$-lacZ fusion with an additional adenine nucleotide between $\mathrm{G}_{3}$ and $\mathrm{U}_{4}$ of the RAT. This insertion is predicted to result in the formation of an extra A:U base pair in the second paired region. Concomitantly, the mutant loop 1 would contain three bases instead of four. Thus, the structure of this RAT is expected to be similar to those of the sucrose and $\beta$-glucoside classes (see Fig. 4.6). 


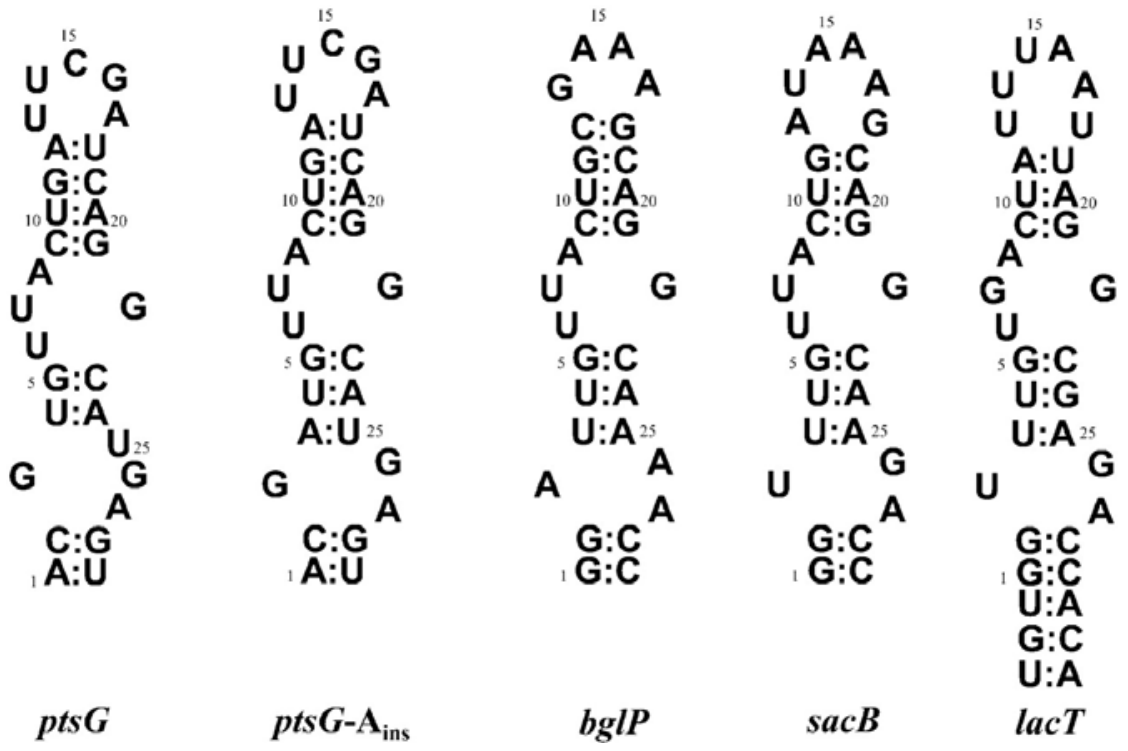

Figure 4.6. Proposed secondary structure for different RAT RNAs (Yang et al., 2002). The fold proposed for the $p t s G-\mathrm{A}_{\text {ins }}$ RAT is based on similarity to the $b g l P$ and $s a c B$ RAT RNAs.

Binding of antitermination proteins to this mutant RAT was assayed by determining the activity of the ptsG-lacZ fusion under conditions when the different antiterminator proteins are known to be active. In the wild type strain QB5448, glucose, sucrose and salicin induced expression of the fusion. This finding is in agreement with previous reports and results from the activity of GlcT due to non-specific regulation by glucose permease (compare QB5448 and GP109 in Table 9) (Görke et al., 2004; Langbein et al., 1999; Stülke et al., 1997). In contrast, the mutant $p t s G$ control region present in GP385 did not allow induction by glucose, suggesting that the altered RAT was not recognized by GlcT. However, significant induction was observed in the presence of sucrose or the $\beta$-glucoside salicin. This induction was independent of a functional glcT gene (compare GP385 and GP386 in Table 9), suggesting that the mutant RAT was bound by a different antiterminator. To test this possibility, we constructed strains carrying the wild type or mutant RAT sequences and deletions of the genes encoding the other B. subtilis antiterminators of the BglG family, i.e. sacT, sacY and licT. These deletions had no effect on the activity of a $p t s G-l a c Z$ fusion in the presence of the wild type RAT sequence. This observation is in good agreement with the idea that induction of pts $G$ by sucrose and salicin is mediated by GlcT (Görke et al., 2004; Langbein et al., 1999; see above). However, the lacZ fusion controlled by the mutant RAT was not induced by sucrose and salicin in the licT mutant strain GP390. Thus, LicT is essential to overcome 
transcription termination in the presence of the altered RAT sequence. Similarly, induction by sucrose is lost in the sacT sacY double mutant strain GP388. Sact and SacY recognize identical RAT sequences and therefore the effect of these mutations was not assayed individually. Interestingly, induction is still possible in the presence of salicin in GP388, confirming that this induction is caused by LicT.

\section{Discussion}

As a target of regulation, RNA differs substantially from DNA in its structural diversity and in its ability to adopt alternative, mutually exclusive structures. Moreover, these structures, the riboswitches, are capable of interacting with a wide variety of regulatory partners, such as metabolites, other RNAs or proteins. Due to this huge variability, the themes in RNA-protein recognition are much more diverse than those identified in DNA-protein interactions.

GlcT and the pts $G$ riboswitch are members of an expanding family of transcription regulatory systems (Stülke et al., 1998). A recent analysis of the evolution of the regulatory domains of the antitermination proteins of this family revealed that GlcT from B. subtilis and its ortholog from $S$. carnosus form a distinct subgroup whereas the antiterminators of the sucrose and ß-glucoside classes exhibit a close relationship with each other (Greenberg et al., 2002). This is supported by the finding that there is no cross-talk between the regulatory components of the glucose type on the one hand and those of the sucrose and $\beta$-glucoside type on the other (Langbein et al., 1999). In contrast, binding of sucrose antiterminator proteins to RAT sequences of $b g l$ genes was reported (Aymerich and Steinmetz, 1992).

Our studies regarding the overall structure of the pts $G$ RAT suggest that it folds similarly to the RAT RNAs of the $b g l$ and $s a c$ classes, thus confirming a proposal of Yang et al. (Yang et al., 2002). Interestingly, this structure deviates from that obtained by the Zuker algorithm proposed earlier (Langbein et al., 1999). However, both mutational and footprint analyses are in disagreement with the RAT structure suggested previously, whereas they confirm the model depicted here (see Figs. 4.1, 4.6, and 4.7). Mutations in the proposed stem regions had different consequences: single mutations prevented antitermination while compensatory mutations that restored the base pairing restored GlcT binding at the same time. Similar results were obtained with the $s a c B$ RAT sequence (Aymerich and Steinmetz, 1992). 


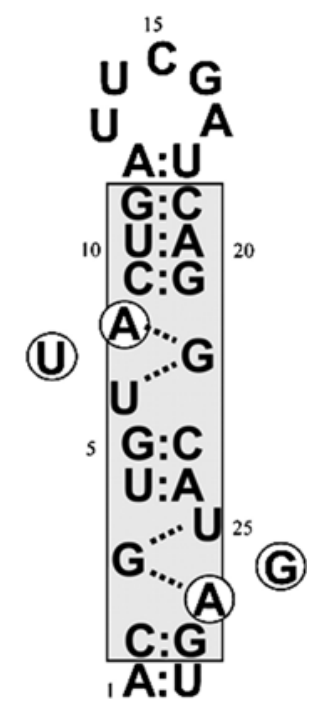

Figure 4.7. Proposed model of the base pairing in the RAT RNA. The circled bases are proposed to directly interact with GlcT.

While the sequence of the ptsG RAT differs substantially from those of the RAT sequences of the $s a c$ and $b g l$ classes, we detected a perfect match of loop 2 of the $p t s G, b g l P$ and $s a c B$ RAT sequences and the regions surrounding loop 2 (see Fig. 4.6). It therefore seems safe to conclude that this region of the RAT is structurally identical to that of the bglP RAT as determined by NMR spectroscopy (Yang et al., 2002). In loop 2, $\mathrm{G}_{22}$ forms contacts with two bases, $\mathrm{U}_{6}$ and $\mathrm{A}_{8}$. Thus, only $\mathrm{U}_{7}$ extrudes from the stem structure (see Fig. 4.7). Analysis of the LicT interaction with the $b g l P$ RAT revealed that $\mathrm{G}_{22}$ is not involved in direct interactions with the protein. However, the mutations of $\mathrm{G}_{22}$ and its partners studied in this work abolished antitermination. This confirms the critical importance of these residues for the structure of the RAT. The pts $G$ RAT is unique since it is the only RAT sequence with a symmetrical arrangement of the two loops. Therefore, it is tempting to speculate that $\mathrm{G}_{3}$ is involved in contacts with $U_{25}$ and $A_{27}$, with $G_{26}$ being exposed. This idea is again supported by the mutational analysis: mutations disrupting the 'ménage à trois' in loop 1 strongly interfere with antitermination and are therefore expected to interfere with the RAT structure, as observed for loop 2.

Our studies concerning the interaction of GlcT with the RAT revealed that loops 1 and 2, as well as the stems, are critical for antitermination. This is in good agreement with previous findings for the SacY-sacB RAT and LicT-bglP RAT interactions (Aymerich and Steinmetz, 1992; Yang et al., 2002). Our mutation and footprint analyses demonstrated that the top loop is important neither for adoption of the proper RAT structure nor for the 
interaction with GlcT. In contrast, the RNA-binding coat protein of bacteriophage MS2 and the phage $\lambda \mathrm{N}$ protein requires the top loop of their RNA targets for binding (Grahn et al., 1999; Legault et al., 1998). As far as the paired regions are concerned, our analysis fails to identify the bases and amino acids in the RAT and GlcT, respectively, that are directly involved in the interaction. However, in our footprint analysis $G_{26}$ in loop 1 was strongly protected from RNase T1 cleavage in the presence of GlcT, suggesting that it is contacted by the protein. The corresponding base in loop 2 was shown to be involved in interaction of the bglP RAT with several amino acids of LicT (Yang et al., 2002). We have analyzed mutations of these two exposed nucleotides in loop $1\left(\mathrm{G}_{26}\right)$ and loop $2\left(\mathrm{U}_{7}\right)$. In both cases a strong induction by glucose was observed. We may therefore propose that, although these exposed bases are contact partners for GlcT, their actual nature does not seem to be crucial. This fits well with previous observations on the interaction of the bglP RAT and LicT (Yang et al., 2002).

The idea that the structure of the RAT rather than its sequence is important for recognition by GlcT is strongly supported by the observation that insertion of a single nucleotide that makes the RAT structure more similar to that of the $b g l$ and $s a c$ RATs resulted in complete loss of GlcT-dependent induction. In contrast, the novel RAT is recognized by LicT even though the sequence remains quite different from that of the cognate LicT RAT sequences. On the other hand, a small sequence alteration with a probably important structural consequence results in complete loss of antitermination by GlcT. So far, it is known that SacT and SacY bind to RAT sequences of the $b g l$ and $s a c$ classes whereas LicT (and its E. coli counterpart BglG) binds only to bgl RAT sequences (Aymerich and Steinmetz, 1992; Manival et al., 1997). The structures of the RAT sequences of these two classes are very similar (see Fig. 4.6). Accordingly, the recognition specificity for LicT is determined in the sequence of the RAT, and mutations in loop 1 of the $s a c B$ RAT sequence allowed binding of BglG in addition to SacY. The effects of the corresponding mutations were cumulative (Aymerich and Steinmetz, 1992). This is in agreement with the idea that SacY and LicT recognize RAT RNAs with the same structure. In contrast, we report here the first example of a complete change of partner specificity. A single mutation that changes the structure of the RAT RNA is sufficient for altered recognition and results in an all-or-nothing effect. Obviously, a RAT can be a member of the $g l c$ or the sac/bgl family, with no intermediate possible. The RNAbinding domains of LicT and GlcT are well conserved (for alignments see Langbein et al., 1999; Yang et al., 2002). Five amino acids of LicT were shown to be involved in the direct interaction with the internal loops of the RAT RNA (Yang et al., 2002). Of these, three (N10, 
G26 and F31) are conserved in all B. subtilis antiterminator proteins of this family. The residue $\mathrm{K} 5$ is conserved in all proteins but SacY. Only R27 is not conserved at all and may thus be involved in providing interaction specificity.

The evolution of regulation by adaptation of DNA-binding proteins to new DNAbinding sites is well documented. In the case of protein-dependent riboswitches, the question arises what was first: does the protein evolve to recognize an altered RNA or does the RNA adapt to protein variations? Our results hint at the second idea: one mutation in the RNA is sufficient to gain a novel regulatory specificity. In contrast, many mutations in the protein would be required to recognize an RNA target with a similar sequence but different structure. It will be interesting to study the molecular details of GlcT-RAT interaction by determining the structure of the complex. 


\section{Keeping signals straight in transcription regulation: specificity determinants for the interaction of a family of conserved bacterial RNA- protein couples}

The results described in this chapter were published in:

Schilling, O., C. Herzberg, T. Hertrich, H. Vörsmann, D. Jessen, S. Hübner, F. Titgemeyer, and J. Stülke. 2006. Keeping signals straight in transcription regulation: specificity determinants for the interaction of a family of conserved bacterial RNA-protein couples. Nucleic Acids Res 34:6102-6115.

Author contributions:

This study was designed by O.S. and J.S.. O.S. performed and interpreted the reporter gene analyses and gel shift experiments. O.S. constructed all mutant strains. S.H. performed the Northern Blot analysis. T.H. performed the glucose uptake measurements and F.T. supervised T.H. during her diploma work. H.V. and D.J. contributed to some of the mutant strain constructions and reporter gene analyses during their practical training under the supervision of O.S.. C.H. contributed substantially to all lab work related to this project. O.S. and J.S. wrote the paper. 


\begin{abstract}
Regulatory systems often evolve by duplication of ancestral systems and subsequent specialization of the components of the novel signal transduction systems. In the Grampositive soil bacterium Bacillus subtilis, four homologous antitermination systems control the expression of genes involved in the metabolism of glucose, sucrose and $\beta$-glucosides. Each of these systems is made up of a sensory sugar permease that does also act as phosphotransferase, an antitermination protein, and a RNA switch that is composed of two mutually exclusive structures, a RNA antiterminator (RAT) and a transcriptional terminator. We have studied the contributions of sugar specificity of the permeases, carbon catabolite repression, and protein-RAT recognition for the straightness of the signaling chains. We found that the $\beta$-glucoside permease BglP does also have a minor activity in glucose transport. However, this activity is irrelevant under physiological conditions since carbon catabolite repression in the presence of glucose prevents the synthesis of the $\beta$-glucoside permease. Reporter gene studies, in vitro RNA-protein interaction analyses and northern blot transcript analyses revealed that the interactions between the antiterminator proteins and their RNA targets are the major factors contributing to regulatory specificity. Both structural features in the RATs and individual bases are important specificity determinants. Our study revealed that the specificity of protein-RNA interactions, substrate specificity of the permeases as well as the general mechanism of carbon catabolite repression together allow to keep the signaling chains straight and to avoid excessive cross-talk between the systems.
\end{abstract}

\title{
Introduction
}

To sense their environment and to adapt to changing conditions, all organisms possess signal transduction systems which are composed of a sensor that perceives the signal, a regulator that can modify its activity in response to the signal, and a target of regulation. This general scheme can be modified in many ways: The sensor and the regulator are often combined in the same molecule as in the Lac repressor. The sensor and the regulator are usually proteins, but regulatory RNAs continue to be uncovered. The target of the regulation may be a protein, i.e. an enzyme, but for the control of gene expression, specific DNA or RNA sequences are the most common targets. 
In bacteria, the number of environmental or internal signals that need to be sensed is much higher than the number of non-related regulatory systems. Thus, large families of regulation systems are present in bacteria. Among the most common families are the twocomponent regulatory systems, sigma factors with their anti-sigma factors as well as several families of repressor and activator proteins (Helmann, 1999; Huffman and Brennan, 2002; Pané-Farré et al., 2005; Ramos et al., 2005; Stock et al., 2000). All these families can be divided into sub-families that do often respond to similar signals. The evolution of signaling families is still in progress and can be observed in the transcriptional regulation of biodegradation pathways. Even more, new regulatory systems can be generated artificially (Galvao and de Lorenzo, 2006; Garmendia et al., 2001). The similarity of the components of many families of signal transduction systems raises the question how the bacteria avoid excessive cross-talk, i.e. the activation of a regulatory protein by gratuitous inducers or the induction of a gene by a non-cognate regulator protein that recognizes a similar DNA sequence. This problem was the subject of extensive analyzes for the two-component regulatory systems in the Gram-positive soil bacterium Bacillus subtilis (Hoch and Varughese, 2001).

We are interested in the control of glucose utilization in B. subtilis. This sugar is transported by a specific permease of the phosphotransferase system (PTS) encoded by ptsG and is subsequently catabolized via the glycolytic pathway (Stülke and Hillen, 2000). The expression of the pts $G$ gene and of several glycolytic genes is inducible by glucose, however, the mechanisms differ. While pts $G$ expression is induced by transcriptional antitermination, the glycolytic gapA operon is controlled by the repressor CggR (Doan and Aymerich, 2003; Fillinger et al., 2000; Ludwig et al., 2001; Stülke et al., 1997). Induction of ptsG expression involves a RNA switch which is the target of the antitermination protein GlcT, and the sensory glucose permease, PtsG. As part of the PTS, the glucose permease possesses two soluble domains that are involved in the phosphate transfer from phosphoenolpyruvate to the incoming sugar, the domains IIA and IIB (Bachem et al., 1997). If glucose is present, the phosphate groups are immediately transferred to the sugar, whereas they accumulate on the glucose permease as well as on the two general proteins of the PTS, enzyme I and HPr, in the absence of glucose. Under these conditions, the glucose permease can transfer a phosphate residue to GlcT thereby inactivating the antitermination protein (Bachem and Stülke, 1998; Schmalisch et al., 2003). GlcT is made up of three domains, an N-terminal RNA-binding domain, and two homologous PTS-regulation domains called PRD-I and PRD-II (Bachem and Stülke, 1998; Manival et al., 1997; Stülke et al., 1998). 


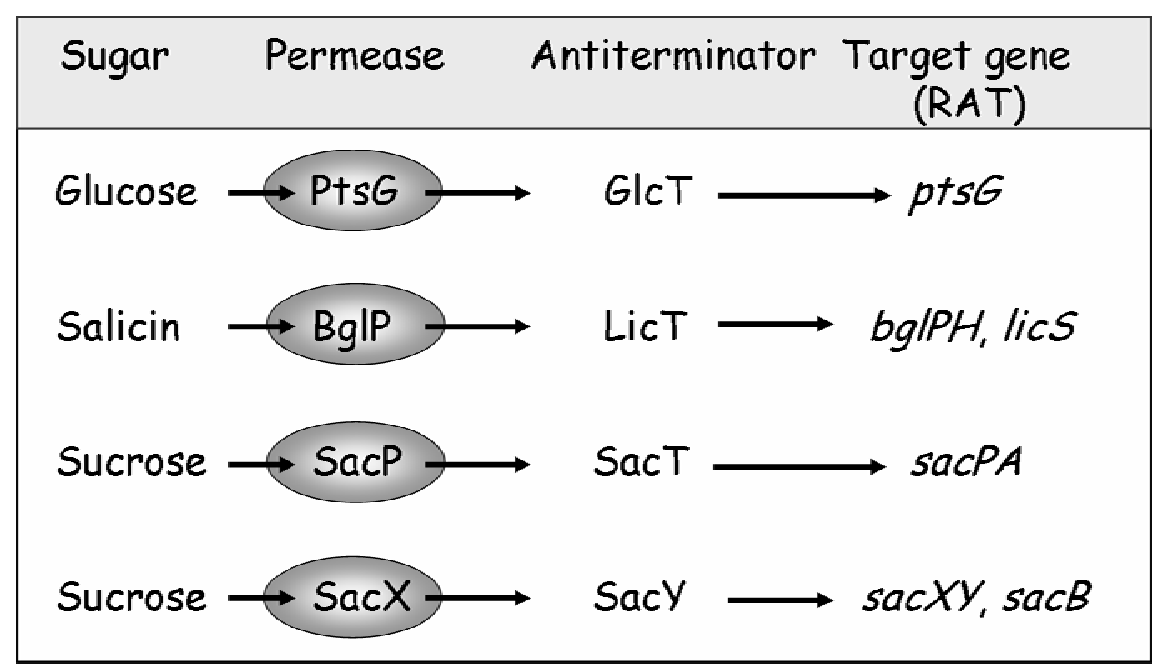

Figure 5.1. A family of antitermination systems controls sugar uptake and metabolism in B. subtilis. The design of the four signaling systems. The target genes encode the following proteins: $p t s G$, glucose permease of the PTS; $b g l P, \beta$-glucoside permease of the PTS; $b g l H$, phospho- $\beta$ glucoside hydrolase; lic $S$, $\beta$-1,3-1,4-glucanase; sacP, sucrose permease of the PTS; sacA, sucrase; $s a c X$, sucrose permease of the PTS (low affinity); $s a c Y$, antitermination protein; $s a c B$, extracellular levansucrase.

Phosphorylation of a conserved histidine residue in PRD-I by the glucose permease results in GlcT inactivation in the absence of glucose. Biochemical studies revealed that PRD-II of GlcT can also be phosphorylated on a conserved histidine residue. However, this phosphorylation is catalyzed by the HPr protein of the PTS and has only a very minor impact on the activity of GlcT (Schmalisch et al., 2003). If in the right phosphorylation state, i.e. if non-phosphorylated in PRD-I, GlcT can bind its target site on the pts $G$ mRNA called RNA antiterminator (RAT) (Aymerich and Steinmetz, 1992; Bachem and Stülke, 1998). The RAT overlaps a transcriptional terminator located in the leader region of the $p t s G$ mRNA and the two structures form a RNA switch since they are mutually exclusive. Binding of GlcT to the RAT is thought to prevent the formation of the terminator and to allow transcription elongation into the pts $G$ structural gene. This regulatory system couples the availability of the inducer glucose to the phosphorylation state of the sensor permease and the antitermination protein GlcT resulting in either of two states of the pts $G$ RNA switch and subsequently in $p t s G$ gene expression.

The regulatory system controlling pts $G$ expression is part of a family made up of highly conserved components, i.e. sensor permeases, antitermination proteins and RAT targets for the regulatory proteins (see Fig. 5.1). The additional permeases transport sucrose and the $\beta$-glucoside salicin. Two antitermination proteins, SacT and SacY, regulate expression of sucrose catabolic genes. While SacT is thought to be active at low sucrose concentrations, high concentrations of sucrose are required to activate SacY since its cognate permease SacX 
has a very weak transport activity (Stülke and Hillen, 2000). LicT controls the expression of the $\operatorname{bglPH}$ operon and the licS gene in the presence of salicin (Schnetz et al., 1996). SacT and LicT are only active if (i) their inducers are present and if (ii) no glucose is present in the medium. This allows their phosphorylation by HPr in the PRD-II leading to activation of the antiterminator proteins.
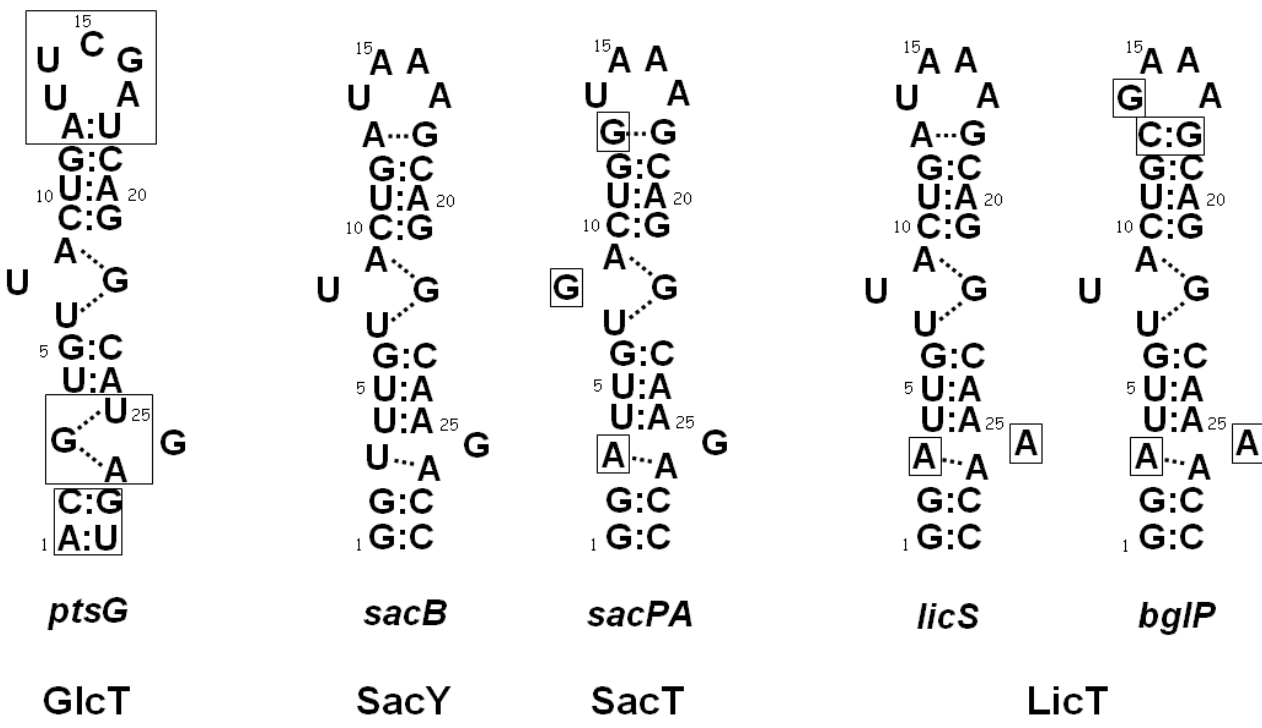

Figure 5.2. Comparison of the secondary structures of the related RAT-RNAs of $B$. subtilis (Schilling et al., 2004; Yang et al., 2002). The relevant antiterminator proteins are indicated below their cognate RAT structures. Boxes indicate nucleotides that differ from the sacB RAT. Dashed lines indicate bases that are proposed to be in direct contact to each other.

In contrast, SacY and GlcT, which are active in the presence of high sugar concentrations (or with the preferred sugar), are independent on a HPr-dependent activation even though HPr can phosphorylate these proteins. The glucose-dependent control of the antiterminator proteins' activity by $\mathrm{HPr}$ is part of the phenomenon of carbon catabolite repression which results in the preferential utilization of easily metabolizable carbon sources (Lindner et al., 1999; Stülke et al., 1998; Tortosa et al., 1997).

The RAT targets of the four antiterminator proteins are all similar to each other (see Fig. 5.2). Some determinants causing specificity of protein-RNA interaction have been identified in a pioneering work (Aymerich and Steinmetz, 1992). The determination of the structure of the complex between the RNA-binding domain of LicT and its cognate RAT RNA suggested that the essential contacts between the protein and the RNA are made in the loop regions of the RAT (Yang et al., 2002). The ptsG RAT recognized by GlcT is most 
different from all other RAT structures, and neither this RAT nor GlcT are involved in any cross-talk (Schilling et al., 2004). In this work, we identified determinants that result in the regulatory specificity of the four distinct antitermination systems.

\section{Materials and Methods}

\section{Bacterial strains and growth conditions}

The B. subtilis strains used in this study are shown in Table 10. All B. subtilis strains are derivatives of the wild type strain 168. Strains used in the cause of site-directed mutagenesis studies are listed in Tables 11 and 12. These strains were all derived by transformation from the basal mutant strains listed in Table 10. Escherichia coli DH5 $\alpha$ and BL21 (DE3) (Sambrook et al., 1989) were used for cloning experiments and for expression of recombinant proteins, respectively.

B. subtilis was grown in SP medium or in CSE minimal medium (Faires et al., 1999). The media were supplemented with auxotrophic requirements (at $50 \mathrm{mg} / \mathrm{l}$ ), carbon sources and inducers as indicated. E. coli was grown in Luria-Bertani medium (LB medium) and transformants were selected on plates containing ampicillin $(100 \mu \mathrm{g} / \mathrm{ml})$. LB and SP plates were prepared by the addition of $17 \mathrm{~g}$ Bacto agar/1 (Difco) to LB or SP medium, respectively.

\section{Transformation and characterization of the phenotype}

B. subtilis was transformed with plasmid DNA according to the two-step protocol described previously (Kunst and Rapoport, 1995). Transformants were selected on SP plates containing kanamycin $(\mathrm{Km} 5 \mu \mathrm{g} / \mathrm{ml})$, chloramphenicol $(\mathrm{Cm} 5 \mu \mathrm{g} / \mathrm{ml})$, spectinomycin (Spc $100 \mu \mathrm{g} / \mathrm{ml})$, or erythromycin plus lincomycin (Em $1 \mu \mathrm{g} / \mathrm{ml}$ and Lin $10 \mu \mathrm{g} / \mathrm{ml})$.

In B. subtilis, amylase activity was detected after growth on SP medium supplemented with $10 \mathrm{~g}$ hydrolyzed starch/1 (Connaught). Starch degradation was detected by sublimating iodine onto the plates.

Quantitative studies of lacZ expression in B. subtilis in liquid medium were performed as follows: cells were grown in CSE medium supplemented with the carbon sources indicated. Cells were harvested at $\mathrm{OD}_{600}$ 0.6-0.8. Cell extracts were obtained by treatment with lysozyme and DNase. $\beta$-Galactosidase activities were determined as previously described using $o$-nitrophenyl-galactoside as a substrate (Kunst and Rapoport, 1995). One unit is defined as the amount of enzyme which produces $1 \mathrm{nmol}$ of $o$-nitrophenol per min at $28{ }^{\circ} \mathrm{C}$. 


\section{DNA manipulation}

Transformation of E. coli and plasmid DNA extraction were performed using standard procedures (Sambrook et al., 1989). Restriction enzymes, T4 DNA ligase and DNA polymerases were used as recommended by the manufacturers. DNA fragments were purified from agarose gels using the QIAquick gel extraction kit (Qiagen ${ }^{\circledR}$, Hilden, Germany). Pfu DNA polymerase was used for the PCR as recommended by the manufacturer.

Table 10. B. subtilis strains used in this study

\begin{tabular}{|c|c|c|}
\hline Strain & Genotype & Source $^{\mathrm{a}}$ \\
\hline 168 & $\operatorname{trp} C 2$ & Laboratory collection \\
\hline BGW10 & trpC2 lys-3 $\Delta l i c T S::$ erm & Krüger and Hecker, 1995 \\
\hline GM1112 & $\begin{array}{l}\text { sacXY } 3 \text { sacB } 23 \text { sacT } 4 \text { bglP::Tn10 erm } \\
\text { amyE::(sacB-lacZ phl) }\end{array}$ & Le Coq et al., 1995 \\
\hline QB5435 & $\operatorname{trp} C 2 \Delta p t s G:: c a t$ & Stülke et al., 1997 \\
\hline QB5448 & 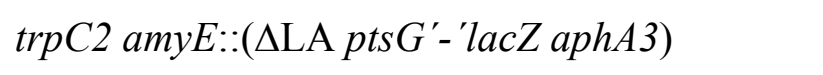 & Stülke et al., 1997 \\
\hline GP109 & 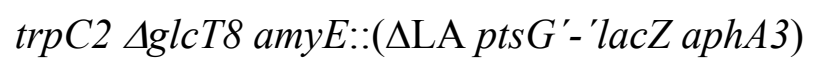 & Bachem and Stülke, 1998 \\
\hline GP150 & $\operatorname{trpC2} \Delta g l c T 8$ amyE::('lacZ cat) & Langbein et al., 1999 \\
\hline GP385 & 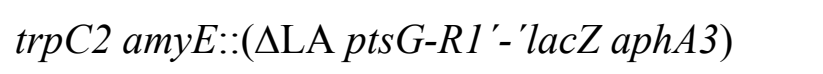 & Schilling et al., 2004 \\
\hline GP425 & $\operatorname{trp} C 2 \Delta s a c Y:: c a t$ & see Materials and Methods \\
\hline GP427 & $\operatorname{trpC2} \Delta$ licTS::erm & $\mathrm{BGW} 10 \rightarrow 168$ \\
\hline GP429 & $\operatorname{trpC2} \Delta s a c T:: s p c$ & see Materials and Methods \\
\hline GP430 & $\operatorname{trp} C 2 \Delta s a c Y:: c a t \Delta s a c T:: s p c$ & GP429 $\rightarrow$ GP425 \\
\hline GP431 & $\operatorname{trpC2} \Delta$ licTS ::erm $\Delta$ sacT::spc & GP429 $\rightarrow$ GP427 \\
\hline GP432 & $\operatorname{trpC2} \Delta$ licTS ::erm $\Delta$ sacY::cat & GP425 $\rightarrow$ GP427 \\
\hline GP433 & $\operatorname{trpC2} \Delta$ licTS ::erm $\Delta$ sacY::cat sacT::spc & GP429 $\rightarrow$ GP432 \\
\hline GP437 & $\operatorname{trpC} 2$ amyE::(sacB-lacZ aphA3) & pGP564 $\rightarrow 168$ \\
\hline GP470 & $\operatorname{trp} C 2 \Delta p t s G:: c a t$ bglP::Tn10 erm & GM1112 $\rightarrow$ QB5435 \\
\hline
\end{tabular}

${ }^{a}$ Arrows indicate construction by transformation.

The combined chain reaction and the multiple mutation reaction were performed with $P f u$ DNA polymerase and thermostable DNA ligase (Ampligase ${ }^{\circledR}$, Epicentre, Wisconsin, USA). DNA sequences were determined using the dideoxy chain termination method (Sambrook et al., 1989). Chromosomal DNA of B. subtilis was isolated as described (Kunst and Rapoport, 1995). 


\section{Construction of sacT and sacY mutant strains by allelic replacement}

To construct $s a c T$ and $s a c Y$ mutant strains, the long flanking homology PCR (LFH-PCR) technique was used (Wach, 1996). Briefly, cassettes carrying the cat and spc resistance genes were amplified from the plasmids pGEM-cat and pDG1726, respectively (Guérout-Fleury et al., 1995; Youngman, 1990). DNA fragments of $\sim 1000 \mathrm{bp}$ flanking the regions to be deleted at their $5^{\prime}$ and $3^{\prime}$ ends were amplified. The $3^{\prime}$ end of the upstream fragment as well as the 5 ' end of the downstream fragment extended into the gene(s) to be deleted in a way that all expression signals of genes up- and downstream of the targeted genes remained intact. The joining of the two fragments to the resistance cassette was performed in a second PCR as described previously (Jordan et al., 2006). In these reaction we used the primer pairs cat-fwd (5'-CGGCAATAGTTACCCTTATTATCAAG)/cat-rev (5'-CCAGCGTGGACCGGCGAGG CTAGTTACCC) and spec-fwd/spec-rev (Jordan et al., 2006) for the amplification and joining of the cat and spc cassettes, respectively. The PCR products were directly used to transform B. subtilis. The integrity of the regions flanking the integrated resistance cassettes was verified by sequencing PCR products of $\sim 1000 \mathrm{bp}$ amplified from chromosomal DNA of the resulting mutants. The resulting strains were GP425 ( $\Delta$ sacY::cat) and GP429 $(\Delta s a c T:: s p c)$.

\section{Site-directed mutagenesis}

Translational fusions of variants of the $p t s G$ and $s a c B$ regulatory regions with the lacZ gene were constructed using the vector pAC7 (Weinrauch et al., 1991) containing the kanamycin resistance gene aphA3. The plasmid harbours a lac $Z$ gene without a promoter located between two fragments of the $B$. subtilis amyE gene. To construct a translational $s a c B$-lacZ fusion the DNA upstream from the $s a c B$ gene $[-464$ to $+15 \mathrm{nt}$ relative to the translational start point of $s a c B$ (Steinmetz et al., 1985)] was amplified by PCR using the primers OS49

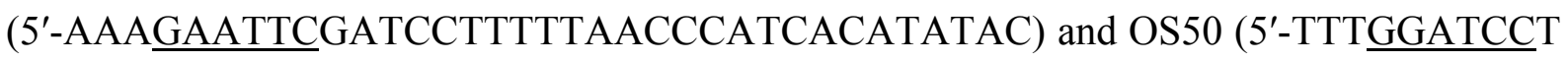
TTTTGATGTTCATCGTTCATGTC). The primers introduced BamHI and EcoRI cloning sites at the ends of the amplified fragment and created an in-frame translational fusion of the lacZ gene with the 5th codon of $s a c B$. The PCR product was inserted into pAC7, both linearized with the same enzymes producing plasmid pGP437.

To study the effect of point mutations in the RAT sequences the following strategy was applied: a DNA fragment carrying the mutant form of the RAT was constructed by sitedirected mutagenesis using either the combined chain reaction or the multiple mutation reaction (to introduce three or more mutations simultaneously) as outlined previously (Bi and 
Stambrook, 1998; Hames et al., 2005). Plasmids pGP66 (Stülke et al., 1997) and pGP437 containing the ptsG and $s a c B$ promoter regions, respectively, served as templates. The mutagenic primers and the resulting plasmids are available upon request. The oligonucleotides JS11 (Stülke et al., 1997)/IL5 (Langbein et al., 1999) and OS49/OS50 (see above) were used as outer primers for $p t s G$ and $s a c B$, respectively. The final PCR products were purified and cut by BamHI and $M f e \mathrm{I}$ (for $p t s G$ ) or BamHI and EcoRI (for $s a c B$ ) sites introduced by the PCR primers. To introduce the constructed lac $Z$ fusions into the chromosome of $B$. subtilis, competent cells of the wild type strain 168 were transformed with the plasmids carrying the respective mutations linearized with ScaI.

\section{Construction of expression vectors for the RNA-binding domains of antiterminator proteins}

A plasmid allowing the fusion of any protein to a Strep tag at the C-terminus was constructed as follows: First, the expression vector pET3C (Novagen) was digested with NdeI and BamHI. The insert containing a small multiple cloning site and the Strep tag was prepared by annealing the complementary oligonucleotides OS91 (5'-TATGGAGCTCGGATCCTGGAG CCACCCGCAGTTCGAAAAATGATAGT) and OS92 (5'-GATCACTATCATTTTTCGA ACTGCGGGTGGCTCCAGGATCCGAGCTCCA). The resulting DNA fragment carries ends compatible with $N d e \mathrm{I}$ and BamHI. Upon ligation, the NdeI site was conserved, whereas the BamHI site was lost. The resulting plasmid, pGP574, carries an IPTG-inducible promoter, a small cloning site (NdeI-SacI-BamHI) for the insertion of the coding sequences, and the sequence encoding the Strep tag followed by two stop codons.

To fuse the RNA-binding domains of GlcT, LicT and SacT to a Strep tag at their C-termini, plasmids pGP575, pGP576 and pGP577 were constructed: DNA fragments corresponding to amino acids 1-60 of GlcT, and 1-57 of LicT and SacT were amplified by PCR using chromosomal DNA of B. subtilis QB5448 and the primer pairs OS93/OS94, OS95/OS96, and OS97/OS98, respectively (the primer sequences are available upon request). The PCR products were digested with $\mathrm{NdeI}$ and $\mathrm{BamHI}$, and the resulting fragments were cloned into the expression vector pGP574 cut with the same enzymes.

\section{Protein purification}

E. coli $\mathrm{BL} 21(\mathrm{DE} 3) / \mathrm{pLysS}$ was used as host for the overexpression of recombinant proteins. Expression was induced by the addition of IPTG (final concentration $1 \mathrm{mM}$ ) to exponentially growing cultures $\left(\mathrm{OD}_{600}\right.$ of 0.8$)$. Cells were lysed using a french press. After lysis the crude 
extracts were centrifuged at $15000 \mathrm{~g}$ for $30 \mathrm{~min}$ and then passed over a Streptactin column (IBA, Göttingen, Germany). The recombinant protein was eluted with desthiobiotin (Sigma, final concentration $2.5 \mathrm{mM}$ ). After elution, the fractions were tested for the desired protein using 12.5\% SDS-PAGE gels. The relevant fractions were combined and dialyzed overnight. Purified proteins were concentrated using Microsep ${ }^{\mathrm{TM}}$ Microconcentrators with a molecular weight cut-off of $3 \mathrm{kDa}$ (Pall Filtron, Northborough, MA). The protein concentration was determined according to the method of Bradford using the Bio-rad dye-binding assay and BSA as the standard.

\section{Assay of interaction between the RNA-binding domains and RAT RNA}

To obtain templates for the in vitro synthesis of the $p t s G$ RAT-RNA, the primers OS25/OS26 (Schilling et al., 2004) were used to amplify a 99 bp PCR product using pGP66 or the plasmid carrying the desired mutation as template. Similarly, a 99 bp DNA fragment encompassing the $s a c B$ RAT was amplified using the oligonucleotides OS86 (5'-CCAAGTAATACGA CTCACTATAGGCGAAAAGTAAATCGCGCG) and OS87 (5'-GTATACACTTTGCCCTT TACAC) and pGP437 or a mutant variant as template. The presence of a T7 RNA polymerase recognition site on primers OS25 and OS86 (underlined) allowed the use of the PCR product as a template for in vitro transcription with T7 RNA polymerase (Roche Diagnostics). The integrity of the RNA transcripts was analyzed by denaturating agarose gel electrophoresis (Ludwig et al., 2001).

Binding of the RNA-binding domains to RAT RNA was analyzed by gel retardation experiments. The RAT RNA (in water) was denatured by incubation at $90{ }^{\circ} \mathrm{C}$ for 2 min and renatured by dilution 1:1 with ice cold water and subsequent incubation on ice. Purified protein was added to the RAT RNA and the samples were incubated for $10 \mathrm{~min}$ at room temperature in TAE buffer in the presence of $300 \mathrm{mM} \mathrm{NaCl}$. After this incubation, glycerol was added to a final concentration of $10 \%(\mathrm{wt} / \mathrm{vol})$. The samples were then analyzed on $10 \%$ Tris-acetate PAA gels.

\section{Northern blot analysis}

RNA was prepared by the modified 'mechanical disruption protocol' described previously (Ludwig et al., 2001). Briefly, $20 \mathrm{ml}$ of cells were harvested at the exponential phase. After mechanical cell disruption, the frozen powder was instantly resuspended in $3 \mathrm{ml}$ lysis buffer [4 $\mathrm{M}$ guanidine isothiocyanate; $0.025 \mathrm{M}$ sodium acetate, $\mathrm{pH} 5.3 ; 0.5 \% \mathrm{~N}$-laurylsarcosine (wt/vol)]. Subsequently, total RNA was extracted using the RNeasy Mini kit (Quiagen, 
Germany). Digoxigenin RNA probes specific for the E. coli lacZ gene were obtained by in vitro transcription with T7 RNA polymerase (Roche Diagnostics) using a PCR-generated fragment as templates. The primers used for PCR were SHU55 (5'-GTTTTACAACGTCG TGACTGG) and SHU56 (5'-CTAATACGACTCACTAT AGGGAGGTGTGCAGTTCAAC CACCG). The reverse primers contained a T7 RNA polymerase recognition sequence. In vitro RNA labeling, hybridization and signal detection were carried out according to the instructions of the manufacturer (DIG RNA labeling kit and detection chemicals; Roche Diagnostics).

\section{Uptake of radioactive glucose in vivo}

B. subtilis strains were grown in CSE medium with glucose $(10 \mathrm{~g} / \mathrm{l})$. Sugar uptake assays were performed as described previously (Stülke et al., 1997). Exponentially growing cells were harvested at an $\mathrm{OD}_{600}$ of $0.6-0.8$ and washed once with the incorporation medium. Labeled $\left[{ }^{14} \mathrm{C}\right]$ glucose $\left(184 \mathrm{mCi} \mathrm{mmol}{ }^{-1}\right)$ and non-labeled glucose (final concentration $0.4 \mathrm{mM}$ ) were added. Samples were taken and treated as described (Stülke et al., 1997).

\section{Results}

\section{Analysis of the loop structures in the ptsG RAT}

The $p t s G$ RAT differs from all other RAT sequences recognized by antiterminator proteins of the $\mathrm{BglG} / \mathrm{SacY}$ family in the structure of the lower loop (see Fig. 5.2). In a previous work, we have demonstrated that the insertion of one base into the lower loop of the pts $G$ RAT (the pts $G$-R1 mutation, see Fig. 5.3) makes its structure similar to that recognized by the other antiterminator proteins and results in exclusive binding of LicT to this structure, whereas it is not bound by GlcT (Schilling et al., 2004). From this result, it was concluded that structure rather than the nucleotide sequence is important for antiterminator protein-RAT recognition.

Since the antitermination proteins bind as dimers to the RAT, and LicT contacts different structures of the lower and the upper loop, we asked whether a RAT with an 'inversion' of the lower and upper RAT structures might be recognized by any of the antitermination proteins (see Fig. 5.3). 


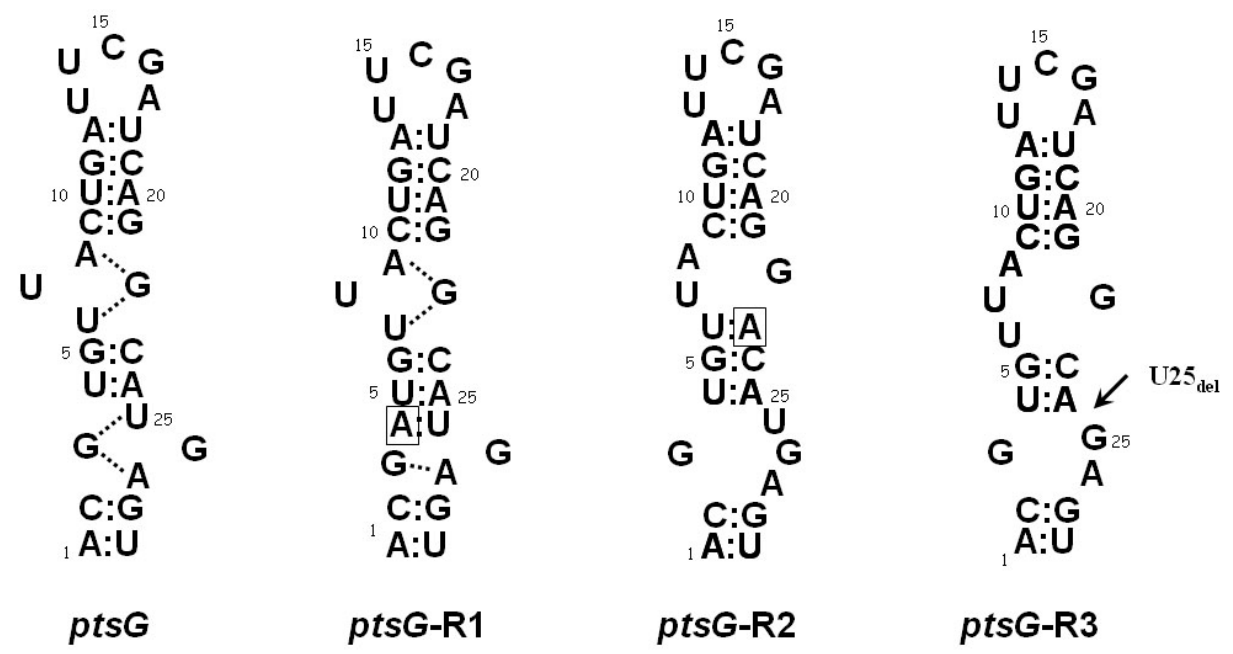

Figure 5.3. Predicted secondary structures of ptsG-RAT and the ptsG-RAT mutants ptsG-R1, ptsG-R2, and ptsG-R3. The insertions of an adenine at position 4 in the $p t s G-\mathrm{R} 1$ and at position 23 in the $p t s G-\mathrm{R} 2$ RAT mutants are boxed. The deletion of the base $\mathrm{U} 25$ in $p t s G-\mathrm{R} 3$ mutant is indicated by an arrow.

The activity of this mutant RAT, ptsG-R2, was assayed by analyzing the expression of a translational fusion of the mutated $p t s G$ control region to a promoterless lac $Z$ gene (see Table 11). While the presence of glucose in the growth medium resulted in a strong GlcT-dependent induction of the pts $G$ promoter in the wild type, salicin induced the $p t s G-\mathrm{R} 1$ promoter region in a LicT-dependent manner. In contrast, the ptsG-R2 promoter region did not allow expression of the lac $Z$ fusion irrespective of the potential inducing carbohydrate present in the medium. Thus, this RAT is not bound by any of the antitermination proteins in B. subtilis (Table 11).

The ptsG-R1 structure was obtained by inserting an A after position 3 of the RAT sequence. This did not only create a lower loop structure similar to those present in RAT structures bound by LicT, SacT, and SacY, but did also generate an additional base pair between the lower and upper loops (see Figs. 5.1, 5.2, and 5.3). To rule out any effect of this extra base pair we constructed the $p t s G$-R3 RAT mutant by deleting the $\mathrm{U}$ at position 25 . This results in a lower loop identical to that in the $p t s G$-R1 RAT, but separated from the upper loop by only 2 bp (Fig. 5.3). 

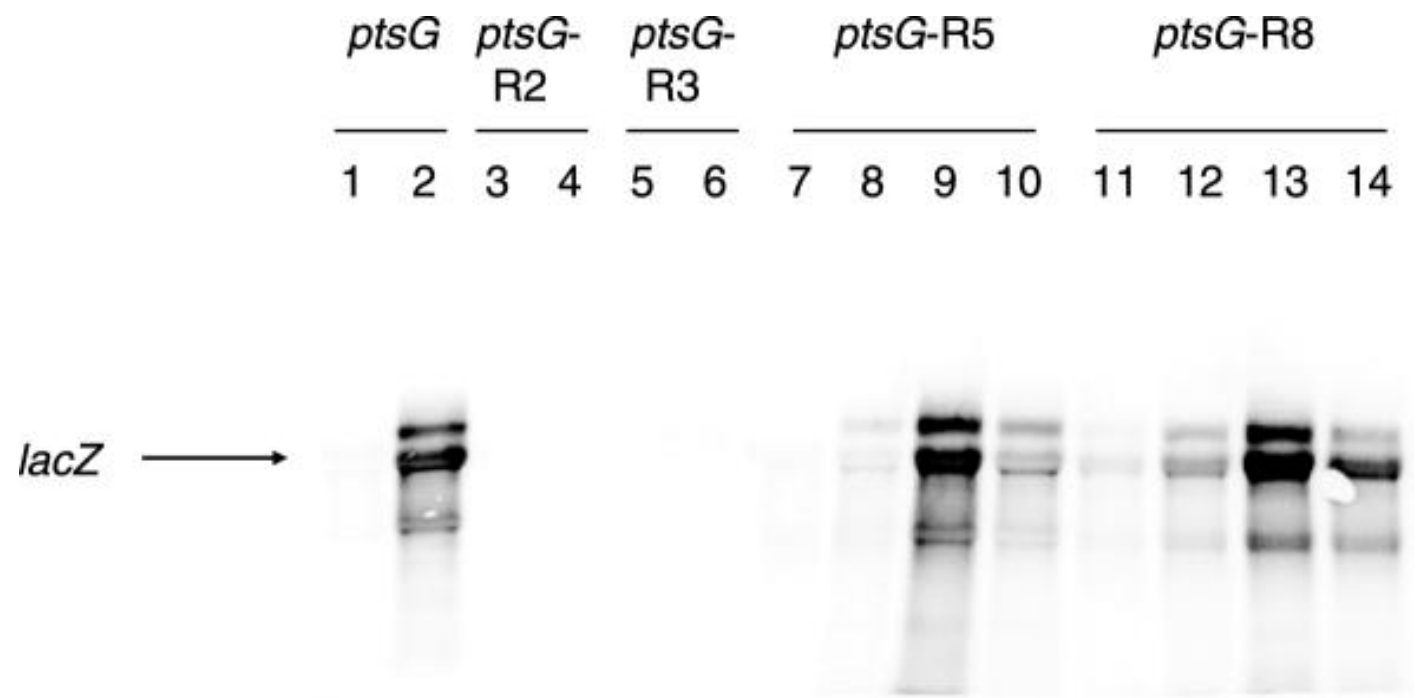

Figure 5.4. Northern Blot analysis of the expression of the lacZ gene under control of wild type and mutant RATs. Total RNA was separated by electrophoresis in $1.0 \%$ agarose gels and, after blotting, nylon membranes were hybridized to a riboprobe specific for lacZ. The mRNA corresponding to the lac $Z$ gene is marked by an arrow. Note that a larger transcript was detected due to imperfect termination of $l a c Z$ transcription. $5 \mu \mathrm{g}$ RNA per lane were applied. The RNAs were isolated from the wild type strain QB5448 (lanes 1, 2), and the RAT mutant strains GP413 (ptsG-R2, lanes 3, 4), GP415 ( $p t s G-\mathrm{R} 3$, lanes 5, 6), GP404 ( $p t s G$-R5, lanes 7 to 10), and GP419 (pts $G$-R8, lanes 11 to 14). The cultures for RNA isolation were grown in CSE minimal medium (lanes $1,3,5,7,11$ ), in CSE medium supplemented with glucose (lanes $2,4,6,8,12$ ), salicin (lanes 9, 13), or sucrose (lanes 10, 14).

The biological activity of this RAT mutant was determined by studying its effect on the expression of a ptsG-lacZ fusion. As shown in Table 11, the ptsG-R3 RAT did not confer induction under any of the conditions tested, suggesting that this structure is not recognized by any of the antitermination proteins (see Discussion). The inability of GlcT to bind to the $p t s G-\mathrm{R} 2$ and $p t s G$-R3 RATs was verified by a northern blot analysis. The amounts of lacZ mRNA were compared in the wild type strain QB5448 and the two mutant strains GP413 and GP415. As can be seen in Figure 5.4, the lacZ mRNA was strongly induced in cells grown in the presence of glucose whereas no induction was observed in the two mutant strains. This result is in perfect agreement with those obtained by the reporter gene assays (Table 11). 
Table 11. Effect of mutations in the pts $G$ RAT on recognition by the different antiterminator proteins.

\begin{tabular}{|c|c|c|c|c|c|c|c|}
\hline \multirow{2}{*}{ Strain } & \multirow{2}{*}{ RAT } & \multirow{2}{*}{ Relevant genotype } & \multicolumn{5}{|c|}{ ß-Galactosidase activity (U/mg protein) } \\
\hline & & & CSE & CSE-Glc & CSE Suc $(0.1 \%)$ & CSE Suc $(2 \%)$ & CSE Sal \\
\hline QB5448 & $p t s G$ & wild type & 9 & 548 & 231 & 329 & 267 \\
\hline GP109 & $p t s G$ & $g l c T$ & 4 & 5 & 2 & 8 & 9 \\
\hline GP387 & $p t s G$ & $\Delta s a c T \Delta s a c Y$ & 10 & 327 & 85 & 166 & 335 \\
\hline GP389 & $p t s G$ & $\Delta l i c T$ & 12 & 412 & 222 & 422 & 312 \\
\hline GP385 & $p t s G-\mathrm{R} 1$ & wild type & 6 & 8 & 34 & 37 & 102 \\
\hline GP386 & $p t s G-\mathrm{R} 1$ & $g l c T$ & 10 & 18 & 48 & 49 & 121 \\
\hline GP390 & $p t s G-\mathrm{R} 1$ & $\Delta l i c T$ & 16 & 11 & 17 & 14 & 13 \\
\hline GP413 & $p t s G-\mathrm{R} 2$ & wild type & 3 & 2 & 2 & 3 & 4 \\
\hline GP415 & $p t s G-\mathrm{R} 3$ & wild type & 14 & 11 & 12 & 7 & 14 \\
\hline GP416 & $p t s G-\mathrm{R} 4$ & wild type & 11 & 3 & 8 & 6 & 94 \\
\hline GP396 & $p t s G-\mathrm{R} 4$ & $g l c T$ & 7 & 9 & 34 & 20 & 90 \\
\hline GP417 & $p t s G-\mathrm{R} 4$ & $\Delta l i c T$ & 10 & 10 & 11 & 10 & 9 \\
\hline GP404 & $p t s G-\mathrm{R} 5$ & wild type & 34 & 33 & 174 & 121 & 877 \\
\hline
\end{tabular}




\begin{tabular}{|c|c|c|c|c|c|c|c|}
\hline GP400 & $p t s G-\mathrm{R} 5$ & $g l c T$ & 24 & 100 & 457 & 178 & 633 \\
\hline GP402 & $p t s G-\mathrm{R} 5$ & $\Delta l i c T$ & 12 & 18 & 66 & 80 & 16 \\
\hline GP455 & $p t s G-\mathrm{R} 5$ & $\Delta l i c T \Delta s a c Y$ & 7 & 9 & 42 & 40 & 8 \\
\hline GP454 & $p t s G-\mathrm{R} 5$ & $\Delta l i c T \Delta s a c T$ & 6 & 10 & 10 & 9 & 9 \\
\hline GP456 & $p t s G-\mathrm{R} 5$ & $\Delta l i c T \Delta s a c T \Delta s a c Y$ & 10 & 9 & 10 & 11 & 8 \\
\hline GP434 & $p t s G-\mathrm{R} 5$ & glcT $\Delta l i c T$ & 9 & 8 & - & - & 6 \\
\hline GP436 & $p t s G-\mathrm{R} 5$ & $\operatorname{glcT} \Delta s a c T$ & 32 & 70 & - & - & - \\
\hline GP435 & $p t s G-\mathrm{R} 5$ & $\operatorname{glcT} \Delta s a c Y$ & 33 & 73 & - & - & - \\
\hline GP408 & $p t s G-\mathrm{R} 6$ & wild type & 34 & 16 & 45 & 50 & 702 \\
\hline GP399 & $p t s G-\mathrm{R} 6$ & $g l c T$ & 19 & 50 & 239 & 156 & 514 \\
\hline GP409 & $p t s G-\mathrm{R} 6$ & $\Delta l i c T$ & 14 & 16 & 20 & 20 & 12 \\
\hline GP464 & $p t s G-\mathrm{R} 7$ & wild type & 37 & 32 & 36 & 37 & 60 \\
\hline GP419 & $p t s G-\mathrm{R} 8$ & wild type & 147 & 188 & 157 & 238 & 1038 \\
\hline GP420 & $p t s G-\mathrm{R} 8$ & $g l c T$ & 160 & 174 & 660 & 798 & 786 \\
\hline GP421 & $p t s G-\mathrm{R} 8$ & $\Delta l i c T$ & 209 & 150 & 300 & 287 & 217 \\
\hline
\end{tabular}

${ }^{a}$ Representative values of $l a c Z$ expression. All measurements were performed at least twice. 
Contribution of individual bases to the recognition of the RAT sequence by antitermination proteins

The cognate RATs bound by LicT, SacT, and SacY are very similar to each other both in terms of structure and sequence (see Fig. 5.2). However, the ptsG-R1 RAT is recognized by LicT only and not by SacY or SacT. Therefore, we decided to introduce further mutations into the $p t s G$-R1 RAT that allow the evaluation of the contribution of individual nucleotides to protein-RNA recognition.

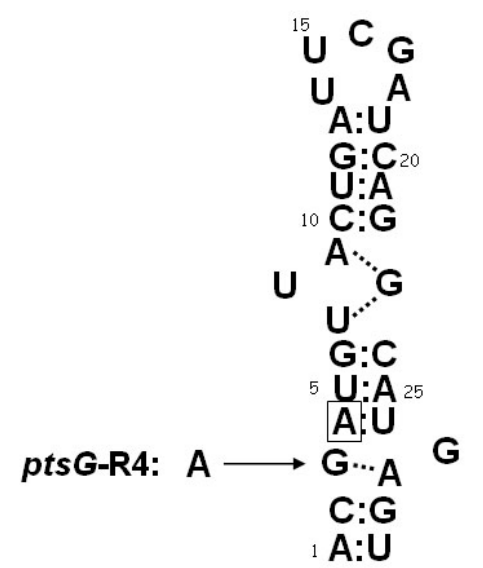

ptsG-R1

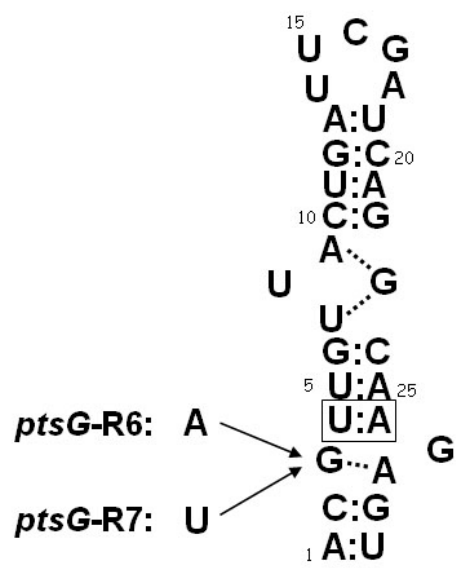

ptsG-R5

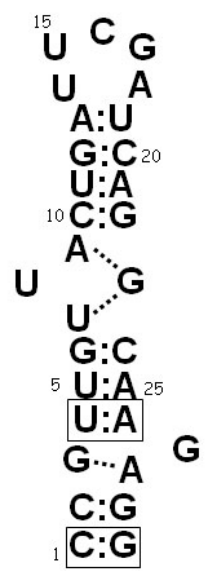

ptsG-R8

Figure 5.5. Secondary structures of the ptsG-R1, ptsG-R5, and ptsG-R8 RAT mutants. Bases that differ from $p t s G$-RAT are boxed.

The RATs recognized by LicT and SacT contain an A at position 3 in the lower loop rather than a $\mathrm{G}$ as in the ptsG-R1 RAT. Therefore, we exchanged the G3 for an A. The effect of this mutation, present in the ptsG-R4 RAT (see Fig. 5.5), was tested by the analysis of a $p t s G-\mathrm{R} 4-l a c Z$ fusion. This mutation resulted in a $l a c Z$ expression comparable to that observed with the ptsG-R1 RAT. As determined for $p t s G-\mathrm{R} 1$, the expression driven by the $p t s G-\mathrm{R} 4$ promoter region was completely dependent on a functional licT gene (Table 11). Thus, LicT is the only antiterminator protein binding to both the $p t s G$-R1 and pts $G$-R4 RATs.

Another important difference between the ptsG-R1 RAT and all other RATs recognized by LicT, SacT, or SacY is the U:A base pair above the lower loop, which is A:U in $p t s G$-R1 (see Figs. 5.2 and 5.3). Previous results suggested that inversions of base pairs in the stems of the RAT are tolerated as long as the general structure is conserved (Schilling et $a l ., 2004)$. However, due to the strict conservation of the U:A pair in this position in all RATs except ptsG-R1, we addressed the effect of such a base pair inversion. As can be seen in Table 11, this inversion, present in the ptsG-R5 mutation (see Fig. 5.5), resulted in an 
increased expression of the fusion under all conditions tested. However, the ptsG-R5 RAT conferred a strong induction in the presence of salicin, and this induction was dependent on the presence of the LicT antiterminator protein. In contrast, glucose did not induce this fusion suggesting that GlcT is unable to bind this RAT (Table 11).

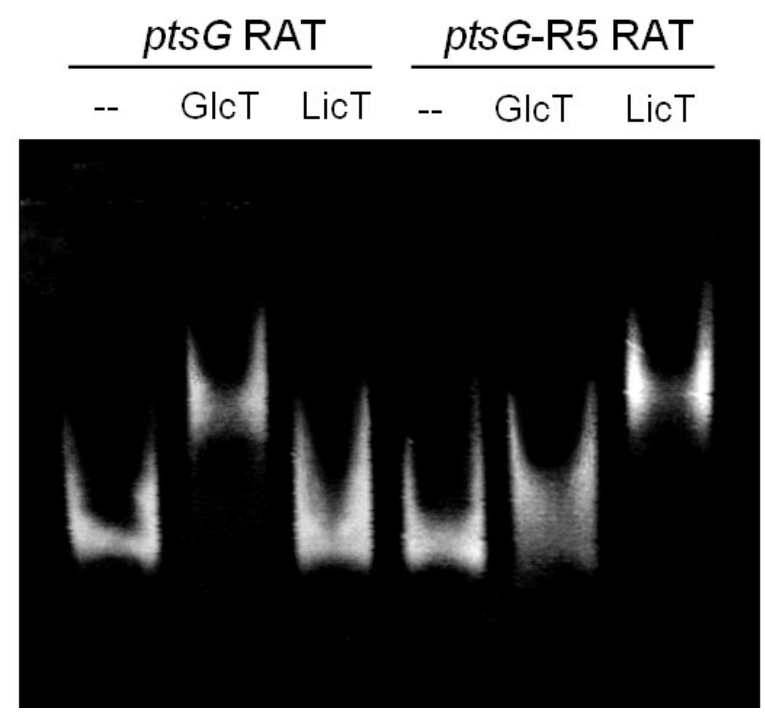

Figure 5.6. Electrophoretic mobility shift analysis of the interaction between the wild type ptsG and ptsG-R5 RATs, and the RNA-binding domains of GlcT or LicT. 100 pmol of the pts $G$ and $p t s G$-R5 RAT RNAs were used. GlcT or LicT ( $250 \mathrm{pmol})$ were added to the RNA as indicated prior to electrophoresis.

These observations were verified by an electrophoretic mobility shift analysis using the purified RNA-binding domains of GlcT and LicT (see Fig. 5.6). As reported previously (Schilling et al., 2004), GlcT efficiently bound the ptsG RAT RNA. In contrast, LicT was unable to bind this RNA. In good agreement with the reporter gene analysis, LicT but not GlcT was capable of binding the ptsG-R5 RAT in vitro (see Fig. 5.6). For the ptsG-R1 RAT, induction in the presence of salicin and, to a lesser extent, sucrose, is strictly LicT-dependent (Table 11) (Schilling et al., 2004). We tested therefore whether the induction of the ptsG-R5 RAT by sucrose was also due to binding of LicT. As mentioned above, a deletion of the licT gene resulted in loss of ptsG-R5 induction by salicin. However, the licT mutation did not abolish the induction by sucrose at the pts $G$-R5 RAT (Table 11) suggesting that either SacT or SacY (or both) bind this RNA and cause antitermination. To test this possibility, we assayed the expression of the ptsG-R5-lacZ fusion in strains containing combinations of mutations of the three antiterminator genes. In a $\operatorname{lic} T$ sacY $Y$ double mutant, a slight reduction of sucrose induction of the ptsG-R5-lacZ fusion was observed as compared to the licT mutant strain (Table 11). 
In contrast, induction was completely lost in the licT sacT and the licT sacT sacY double and triple mutant strains carrying deletions of two and of three antiterminator protein-encoding genes, respectively. From this result we may conclude that SacT can recognize the pts $G$-R5 RAT in addition to LicT. A northern blot analysis of the lacZ mRNA confirmed the strong induction by salicin and, to a lesser extent, by sucrose, conferred by the ptsG-R5 RAT (see Fig. 5.4). Taken together, these data demonstrate that the U:A base pair just above the lower loop is important to facilitate binding of LicT to this structure (compare the high $\beta$-galactosidase activity to that driven by the $p t s G$-R1 fusion, Table 11) and to allow binding of SacT.

The role of the base at position 3 in the lower loop was also analyzed in the context of the $p t s G$-R5 RAT. However, as observed with $p t s G$-R4, only minor effects of a substitution of G3 by A were observed (Table 11, see ptsG-R6, see Fig. 5.5). Induction by salicin was slightly decreased, and the induction with sucrose was also completely dependent on LicT indicating that SacT did not bind the $p t s G$-R6 RAT. A substitution of G3 by U ( $p t s G-\mathrm{R} 7$, as present in the $s a c B$ RAT recognized by SacY, see Fig. 5.5) resulted in loss of induction by sucrose and only weak induction upon the addition of salicin (15-fold reduction as compared to $p t s G$-R5, see Table 11). Taken together, these results indicate that the $\mathrm{G}$ at position 3 facilitates binding of the antitermination proteins. In contrast, an $U$ at this position strongly diminishes binding by LicT. These conclusions are validated by an analysis of the $s a c B$ RAT (see below).

Binding of the antitermination proteins to their RAT targets allows the formation of otherwise non-favored RAT structures and prevents concomitantly the formation of the transcription terminators. The relative stability of the RAT structures may therefore be important for the level of gene expression. The RATs recognized by LicT, SacT, and SacY contain two G:C base pairs in the bottom stem whereas the pts $G$ RAT contains a A:U and a $\mathrm{C}: \mathrm{G}$ base pair at this position. It seemed therefore possible that the replacement of the $A: U$ base pair by a C:G base pair would result in a more stable RAT structure and thus affect transcription. To test this idea, the ptsG-R8 RAT was constructed based on ptsG-R5 and analyzed (see Fig. 5.5, Table 11). While the ptsG-R5 RAT allowed only a weak basal expression in the absence of any inducer (CSE medium), a strongly increased basal expression was found for ptsG-R8 (34 versus $147 \mathrm{U}$ of $\beta$-galactosidase). This was also reflected in a northern blot analysis of lacZ mRNA if expressed under the control of the $p t s G$-R5 and ptsG-R8 RAT (compare Fig. 5.4, lanes 7 and 11). The ptsG-R8-lacZ fusion was also induced by salicin, and the induced expression was the sum of read through $(\sim 150 \mathrm{U})$ and 
real induction ( $\sim 900 \mathrm{U}$, see ptsG-R5, Table 11). However, the increased read through might also result from a destabilization of the terminator even though an extra mutation was introduced in the terminator to restore base pairing.

\section{Carbon catabolite repression interferes with the transport of glucose by BglP}

The analysis of the ptsG-R5 RAT revealed that this structure is efficiently bound by LicT but not by GlcT. The disruption of the glcT gene in a strain carrying the ptsG-R5-lacZ fusion resulted in induction of $\beta$-galactosidase by salicin and sucrose (see Table 11) as expected due to the binding of LicT and SacT, respectively (see above). Surprisingly, glucose did also activate expression of this fusion in a glcT mutant strain. Since GlcT is not available in this mutant, LicT or SacT must be activated in the presence of glucose in the glcT mutant. To test this idea, we studied the activity of the ptsG-R5 control region in glcT licT or glcT sacT double mutants. As shown in Table 11, only a minor effect of the sacT deletion was observed, whereas the deletion of the licT gene resulted in complete loss of induction by glucose. Thus, glucose can activate LicT in a glcT mutant strain.

Two scenarios for the activation of LicT by glucose can be envisaged. First, there might be some non-specificity in BglP that results in the transport of glucose by this permease and the subsequent dephosphorylation and activation of the cognate antiterminator LicT. Second, there might be some cross-talk between the glucose permease PtsG and the LicT antiterminator that results in LicT activation upon glucose transport. Several lines of evidence demonstrate that the former possibility reflects the truth: (i) The glucose permease PtsG is not expressed in a glcT mutant strain and is therefore unable to activate LicT in a glcT mutant (Bachem and Stülke, 1998; Stülke et al., 1997). (ii) BglP phosphorylates and thereby inactivates LicT in the absence of the substrate salicin, and this regulation would be dominant over any minor PtsG-dependent dephosphorylation of LicT (Lindner et al., 1999; Tortosa et al., 1997). (iii) To provide direct evidence for glucose uptake by BglG we measured the glucose transport of glucose-grown cells of a wild type strain (B. subtilis 168), a pts $G$ mutant (QB5435) and a ptsG bglP double mutant (GP470). As shown in Fig. 5.7, glucose was efficiently transported by the wild type strain (initial uptake rate $620 \pm 110$ pmol glucose per minute and $\mathrm{OD}_{600}$ ), whereas a significant reduction was observed in the $p t s G$ mutant (initial uptake rate $62 \pm 5$ pmol glucose per minute and $\mathrm{OD}_{600}$ ). 


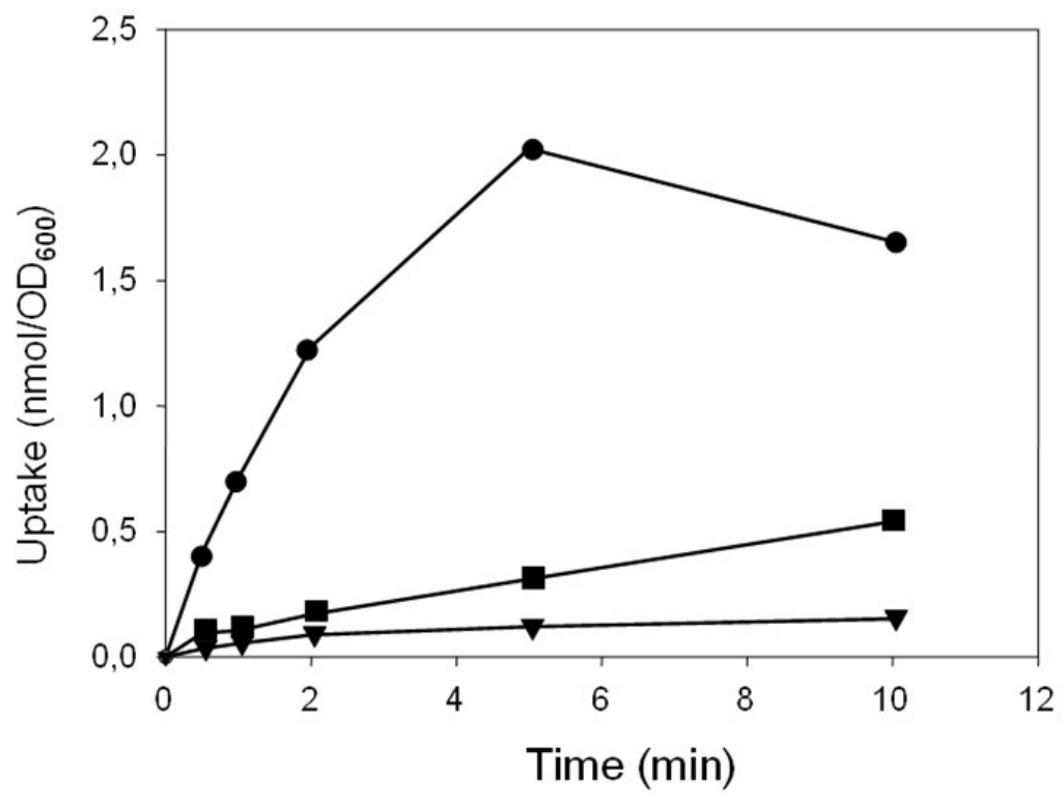

Figure 5.7. The implication of BglP in glucose uptake. For uptake measurements of radioactively labeled glucose the $B$. subtilis strains 168 (wild type, circles), QB5435 (pts $G$, squares), and GP470 (ptsG bglP, triangles) were grown in CSE minimal medium supplemented with $0.5 \%$ of glucose.

These results are in good agreement with previous studies of glucose transport in ptsG mutants (Bachem et al., 1997; Stülke et al., 1997). In the ptsG bglP double mutant GP470, the transport of glucose was further reduced (see Fig. 5.7, initial uptake rate $24 \pm 1$ pmol glucose per minute and $\mathrm{OD}_{600}$ ), confirming that $\mathrm{BglP}$ has some minor glucose transport activity which may explain glucose-dependent activation of LicT in the $g l c T$ mutant background (see Discussion).

\section{Conversion analysis of the $\operatorname{sacB}$ RAT sequence towards new recognition specificities}

The similarity of the ptsG-R6 RAT to that of the $\operatorname{sacPA}$ operon (see Figs. 5.1, 5.2 and 5.5) suggests that both RNA structures might be recognized by the same proteins. However, as shown in Table 11, ptsG-R6 is bound exclusively by LicT whereas the sacPA RAT is the target of SacT and is not recognized by LicT (Manival et al., 1997). Similarly, the ptsG-R7 RAT which is poorly recognized by LicT but by none of the other antiterminator proteins resembles strongly the $s a c B$ RAT which is the target of SacY (see Figs. 5.1, 5.2, and 5.5). Thus, additional components seem to play a role in RAT-antiterminator protein recognition. To unravel these factors, we decided to perform an in-depth conversion analysis of the $s a c B$ RAT to mutate it and shift it gradually to sequences that are not longer recognized by SacB but rather by one of the three other family members. We chose the $s a c B$ RAT for this purpose 
since $s a c B$ lacks any additional regulation by carbon catabolite repression (Steinmetz et al., 1989). This analysis was aimed at the identification of bases that are responsible for the specificity for one or the other antiterminator protein.

\section{Discrimination between SacY and SacT}

First, we determined the regulation mediated by the wild type $s a c B$ RAT. If the lacZ gene was expressed under the control of this RAT, induction was observed only in the presence of sucrose confirming that neither GlcT nor LicT bind the $s a c B$ RAT. Induction by sucrose occurred both at low and high sucrose concentrations which activate SacT and SacY, respectively. Indeed, induction at a low sucrose concentration was lost in the sacT mutant. In the $\operatorname{sac} Y$ mutant strain, induction was still visible at both concentrations suggesting that SacT is active under both conditions. In the $\operatorname{sacT} \operatorname{sacY}$ double mutant, the sacB RAT-terminator couple did not allow induction under all the conditions tested (Table 12).

The $p t s G$-R5 RAT, which is recognized by LicT and SacT, closely resembles the $s a c B$ RAT but contains a $\mathrm{G}$ at position 3 rather than a $\mathrm{U}$ as in the $\operatorname{sacB}$ RAT. We constructed therefore the $s a c B-\mathrm{R} 1$ RAT by replacing $\mathrm{U} 3$ by a G (see Fig. 5.8). This single mutation resulted in a significant specificity shift. The $s a c B$-R1 RAT was not longer a target for SacY, whereas the activation of SacT allowed a higher $\beta$-galactosidase expression as compared to the wild type $s a c B$ RAT (Table 12).
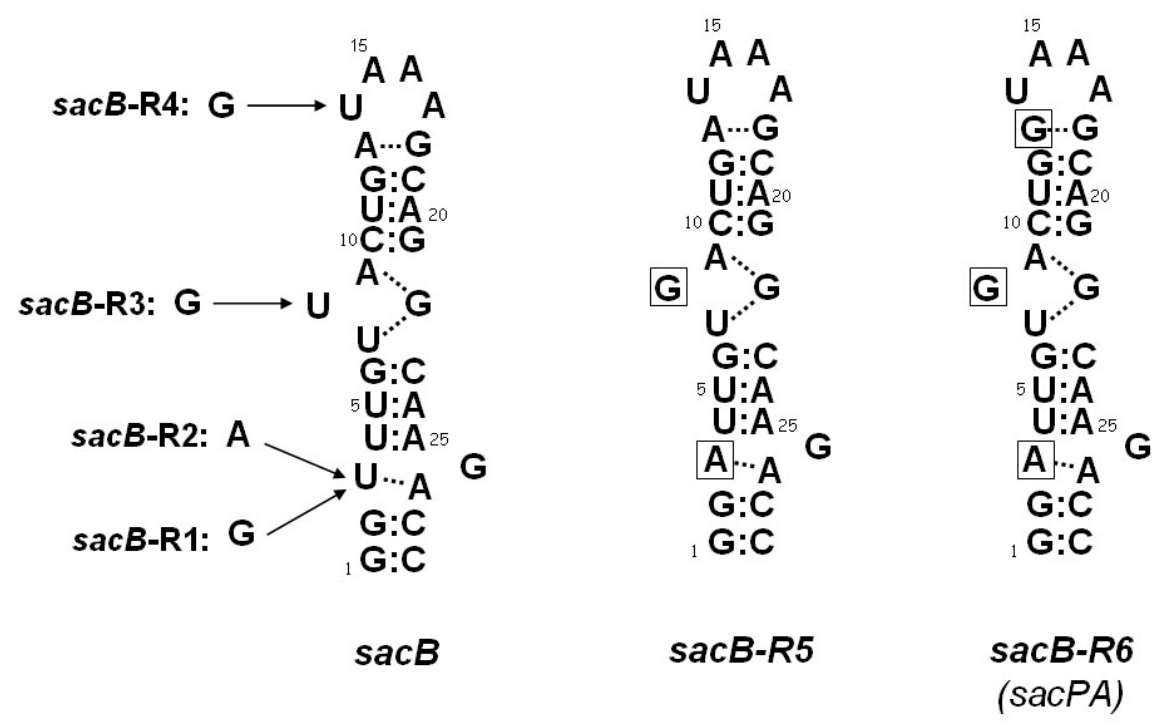

Figure 5.8. Secondary structures of the wild type $\operatorname{sacB}$, $\operatorname{sacB}-\mathrm{R5}$, and $\operatorname{sacB}-\mathrm{R} 6$ mutant RATs. Bases that differ from $s a c B$ RAT are boxed in the $s a c B$-R5 double- and in the $s a c B$-R6 triple-mutant. The $s a c B-\mathrm{R} 1$ to R4 mutant RATs are indicated as single base exchanges into $s a c B$ RAT. The $s a c B-\mathrm{R} 6$ triple mutant RAT is identical to the $s a c P A$ wild type RAT (see Fig. 5.2). 
Moreover, the $s a c B-\mathrm{R} 1$ RAT allowed LicT-dependent induction by salicin. These results are in good general agreement with the observed affinity of the similar ptsG-R5 RAT for SacT and LicT, however, the preference for the two antitermination proteins was inverse. The sacPA RAT, which is the cognate target of SacT, contains also a purine base at position 3, i.e. an A (see Fig. 5.2). Therefore, the $s a c B-\mathrm{R} 2$ containing an A at position 3 was constructed (see Fig. 5.8). The presence of this RAT conferred induction by sucrose but neither by salicin nor glucose (Table 12). Thus, this RAT is not bound by LicT. To distinguish whether it is recognized by SacY or SacT we analyzed the expression driven by the $s a c B-\mathrm{R} 2-l a c Z$ fusion in licT sacT and licT sacY double mutant strains. As shown in Table 12, the sacB-R2 RAT is efficiently bound by SacT at both low and high sucrose concentrations whereas it is not recognized by SacY. The results obtained with the $s a c B-\mathrm{R} 1$ and $-\mathrm{R} 2$ mutants suggest that the $\mathrm{U}$ at position 3 of the RAT is important for recognition by SacY. In contrast, SacT tolerates all three tested bases at this position.

The data presented above demonstrate that U3 is important for SacY binding in the context of the $\operatorname{sacB}$ RAT. However, since SacY is capable of recognizing the $\operatorname{sacPA}$ RAT (Manival et al., 1997), it seems to be able to accept bases different from $U$ at position 3. To address this question we exchanged the three bases in the $s a c B$ RAT that are different from the $\operatorname{sacPA}$ RAT. A replacement of $\mathrm{U} 8$ in the middle loop by a $\mathrm{G}$ as in $\operatorname{sacPA}$ ( $\operatorname{sacB}-\mathrm{R} 3$, see Fig. 5.8) resulted in loss of binding by SacY whereas SacT bound this RAT as judged from loss of sucrose induction in the sacT mutant (see Table 12). Both the $s a c B$ and $s a c P A$ RATs contain a UAAA tetraloop at the top. This loop is flanked by A-G and G-G pairs in $\operatorname{sacB}$ and $\operatorname{sacP} A$, respectively (see Fig. 5.2). Therefore, we constructed the $s a c B$-R4 mutant RAT with a G-G pair at the bottom of the top loop (A13G exchange, see Fig. 5.8). This mutation did not affect binding by SacY and SacT as compared to the wild type $\operatorname{sacB}$ RAT. Moreover, it did not confer induction by salicin indicating that it is no target for LicT (see Table 12). A combination of the two mutations of $s a c B-\mathrm{R} 2$ and $s a c B-\mathrm{R} 3$ (U3A and $\mathrm{U} 8 \mathrm{G}$ ) present in the $s a c B$-R5 RAT (Fig. 5.8) resulted in enhanced induction by sucrose which was exclusively dependent on SacT as determined using a sacT mutant strain (Table 11). Indeed, the $s a c B$-R5 RAT was efficiently bound by the RNA-binding domain of SacT (see Fig. 5.9). 
Table 12. Conversion analysis of the $s a c B$-RAT.

\begin{tabular}{|c|c|c|c|c|c|c|c|}
\hline \multirow{2}{*}{ Strain } & \multirow{2}{*}{ RAT } & \multirow{2}{*}{ Relevant genotype } & \multicolumn{5}{|c|}{ B-Galactosidase activity $(\mathrm{U} / \mathrm{mg} \text { protein })^{\mathrm{a}}$} \\
\hline & & & CSE & CSE Glc & CSE Suc $(0.1 \%)$ & CSE Suc $(2 \%)$ & CSE Sal \\
\hline GP437 & $s a c B$ & wild type & 9 & 6 & 54 & 78 & 5 \\
\hline GP440 & $s a c B$ & $\Delta s a c T$ & 5 & 4 & 5 & 28 & 4 \\
\hline GP438 & $s a c B$ & $\Delta s a c Y$ & 6 & 5 & 50 & 49 & 6 \\
\hline GP441 & $s a c B$ & $\Delta s a c T \Delta s a c Y$ & 6 & 3 & 5 & 4 & 3 \\
\hline GP461 & $s a c B-\mathrm{R} 1$ & wild type & 7 & 8 & 132 & 104 & 35 \\
\hline GP465 & $s a c B-\mathrm{R} 1$ & $\Delta s a c T \Delta l i c T$ & 5 & 4 & 3 & 4 & 2 \\
\hline GP466 & $s a c B-\mathrm{R} 1$ & $\Delta s a c Y \Delta l i c T$ & 5 & 5 & 80 & 74 & 3 \\
\hline GP463 & $s a c B-\mathrm{R} 1$ & $\Delta s a c T \Delta s a c Y$ & 11 & 8 & 10 & 12 & 28 \\
\hline GP462 & $s a c B-\mathrm{R} 1$ & $\Delta l i c T$ & 7 & 9 & 85 & 82 & 5 \\
\hline GP460 & $s a c B-\mathrm{R} 2$ & wild type & 7 & 6 & 93 & 145 & 8 \\
\hline GP472 & $s a c B-\mathrm{R} 2$ & $\Delta s a c Y \Delta l i c T$ & 3 & 5 & 97 & 112 & 5 \\
\hline GP471 & $s a c B-\mathrm{R} 2$ & $\Delta s a c T \Delta l i c T$ & 4 & 3 & 4 & 7 & 3 \\
\hline GP519 & $s a c B-\mathrm{R} 3$ & wild type & 5 & 2 & 96 & 88 & 4 \\
\hline GP521 & $s a c B-\mathrm{R} 3$ & $\Delta s a c T$ & 4 & - & 4 & 9 & 4 \\
\hline GP540 & $s a c B-\mathrm{R} 4$ & wild type & 8 & 8 & 70 & 117 & 7 \\
\hline GP541 & $s a c B-\mathrm{R} 4$ & $\Delta s a c T$ & 7 & 8 & 9 & 35 & 7 \\
\hline GP542 & $s a c B-\mathrm{R} 4$ & $\Delta s a c T \Delta s a c Y$ & 4 & 3 & 3 & 4 & 2 \\
\hline GP520 & $s a c B-\mathrm{R} 5$ & wild type & 4 & 3 & 216 & 208 & 6 \\
\hline GP522 & $s a c B-\mathrm{R} 5$ & $\Delta s a c T$ & 4 & 2 & 5 & 7 & 2 \\
\hline
\end{tabular}




\begin{tabular}{|c|c|c|c|c|c|c|c|}
\hline GP537 & $s a c B-\mathrm{R} 6$ & wild type & 4 & 6 & 349 & 463 & 11 \\
\hline GP538 & $s a c B-\mathrm{R} 6$ & $\Delta s a c T$ & 6 & 5 & 6 & 22 & 9 \\
\hline GP539 & $s a c B-\mathrm{R} 6$ & $\Delta s a c T \Delta s a c Y$ & 3 & 4 & 4 & 4 & 3 \\
\hline GP476 & $s a c B-\mathrm{R} 7$ & wild type & 3 & 6 & 176 & 145 & 92 \\
\hline GP544 & $s a c B-\mathrm{R} 7$ & $\Delta s a c T$ & 5 & 4 & 7 & 8 & 136 \\
\hline GP484 & $s a c B-\mathrm{R} 7$ & $\Delta l i c T$ & 3 & 2 & 195 & 185 & 2 \\
\hline GP536 & $s a c B-\mathrm{R} 7$ & $\Delta s a c T \Delta l i c T$ & 2 & 2 & 3 & 5 & 2 \\
\hline GP477 & $s a c B-\mathrm{R} 8$ & wild type & 6 & 7 & 248 & 294 & 218 \\
\hline GP486 & $s a c B-\mathrm{R} 8$ & $\Delta l i c T$ & 2 & 1 & 305 & 188 & 2 \\
\hline GP487 & $s a c B-\mathrm{R} 8$ & $\Delta s a c T$ & 5 & 4 & 9 & 8 & 376 \\
\hline GP480 & $s a c B-\mathrm{R} 9$ & wild type & 7 & 11 & 280 & 253 & 510 \\
\hline GP492 & $s a c B-\mathrm{R} 9$ & $\Delta l i c T$ & 3 & 4 & 291 & 189 & 3 \\
\hline GP493 & $s a c B-\mathrm{R} 9$ & $\Delta l i c T \Delta s a c T$ & 4 & 2 & 3 & 4 & 2 \\
\hline GP494 & $s a c B-\mathrm{R} 9$ & $\Delta l i c T \Delta s a c Y$ & 3 & 3 & 213 & 193 & 3 \\
\hline GP444 & $s a c B-\mathrm{R} 10$ & wild type & 6 & 3 & 24 & 18 & 10 \\
\hline GP543 & $s a c B-\mathrm{R} 10$ & $\Delta s a c T$ & 5 & - & 5 & 8 & 12 \\
\hline GP446 & $s a c B-\mathrm{R} 11$ & wild type & 48 & 32 & 43 & 34 & 23 \\
\hline GP448 & $s a c B-\mathrm{R} 12$ & wild type & 5 & 4 & 4 & 6 & 4 \\
\hline GP450 & $s a c B-\mathrm{R} 13$ & wild type & 28 & 95 & 112 & 129 & 33 \\
\hline GP453 & $s a c B-\mathrm{R} 13$ & $\Delta l i c T \Delta s a c T \Delta s a c Y$ & 48 & 86 & 104 & 80 & 28 \\
\hline GP451 & $s a c B-\mathrm{R} 13$ & $g l c T$ & 31 & 33 & 20 & 36 & 18 \\
\hline
\end{tabular}

${ }^{a}$ Representative values of $l a c Z$ expression. All measurements were performed at least twice. 
In contrast, the wild type $s a c B$ RAT was only weakly bound by SacT. These observations are in very good agreement with the high SacT-dependent induction of gene expression mediated by $s a c B-\mathrm{R} 5$ as compared to induction conferred by the wild type $s a c B$ RAT. An additional mutation of the base pair at the bottom of the top loop (A13G) made the resulting $s a c B-\mathrm{R} 6$ RAT identical to that of $s a c P A$, but in a sequence context of $s a c B$ (see Figs. 5.2 and 5.8).

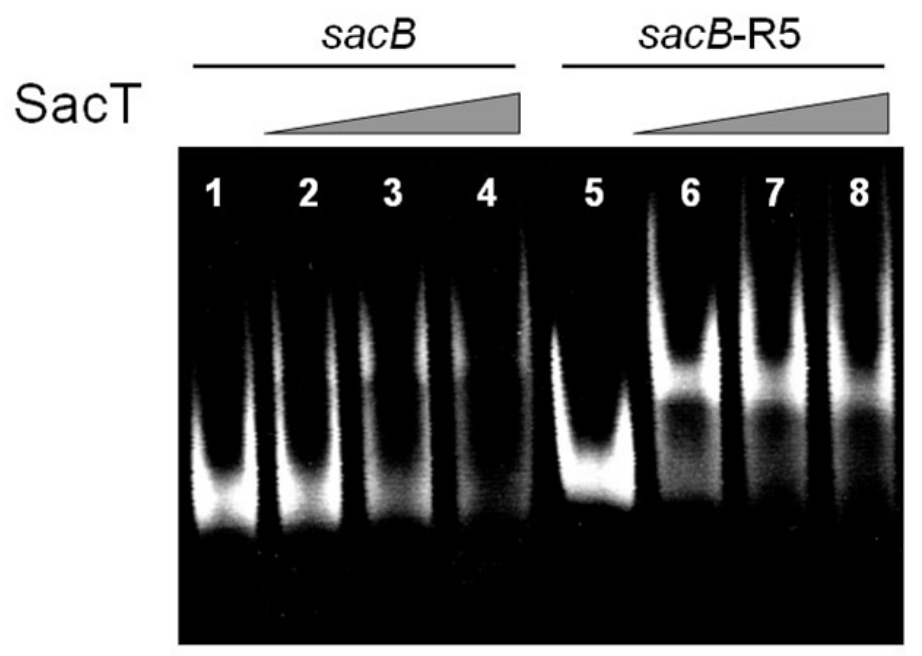

Figure 5.9. Electrophoretic mobility shift analysis of the interaction between the $\operatorname{sac} B$ and sacB-R5 RATs, and the RNA-binding domain of SacT. Lanes 1-4 and 5-8 contain 100 pmol of $s a c B$ and $s a c B$-R5 RAT RNAs, respectively. Increasing concentrations of SacT were added to the RNA in lanes 2-4 and 6-8 prior to electrophoresis. Aliquots of 75, 150 and 300 pmol SacT were used.

As expected, this RAT is most efficiently recognized by SacT. In the sacT mutant, only a very weak induction by sucrose was observed which was lost in the $\operatorname{sacT}$ sacY double mutant strain (Table 12). Thus, the A3 and G8 do both discriminate against binding by SacY. However, as demonstrated using the $s a c B$-R6 RAT, the G-G base pair at the bottom of the top loop seems to weaken this discrimination and does thus allow weak binding by SacY.

\section{Discrimination between SacY and LicT}

The $s a c B$ RAT differs from the licS and bglPH RATs that are the cognate targets of LicT by two bases in the lower loop. Additionally, the bglP RAT contains a C-G base pair at the bottom of the top loop and a GAAA tetraloop at the top (see Fig. 5.2). The first step in the conversion of the $s a c B$ RAT to a structure expected to be recognized by LicT was the $s a c B-\mathrm{R} 2$ mutation (U3A) described above. This RAT was bound by SacT but not by LicT (see Table 12). With the introduction of a second mutation in the lower loop (G26A) the resulting $s a c B$-R7 RAT was identical to that of licS (see Figs. 5.2 and 5.10). 


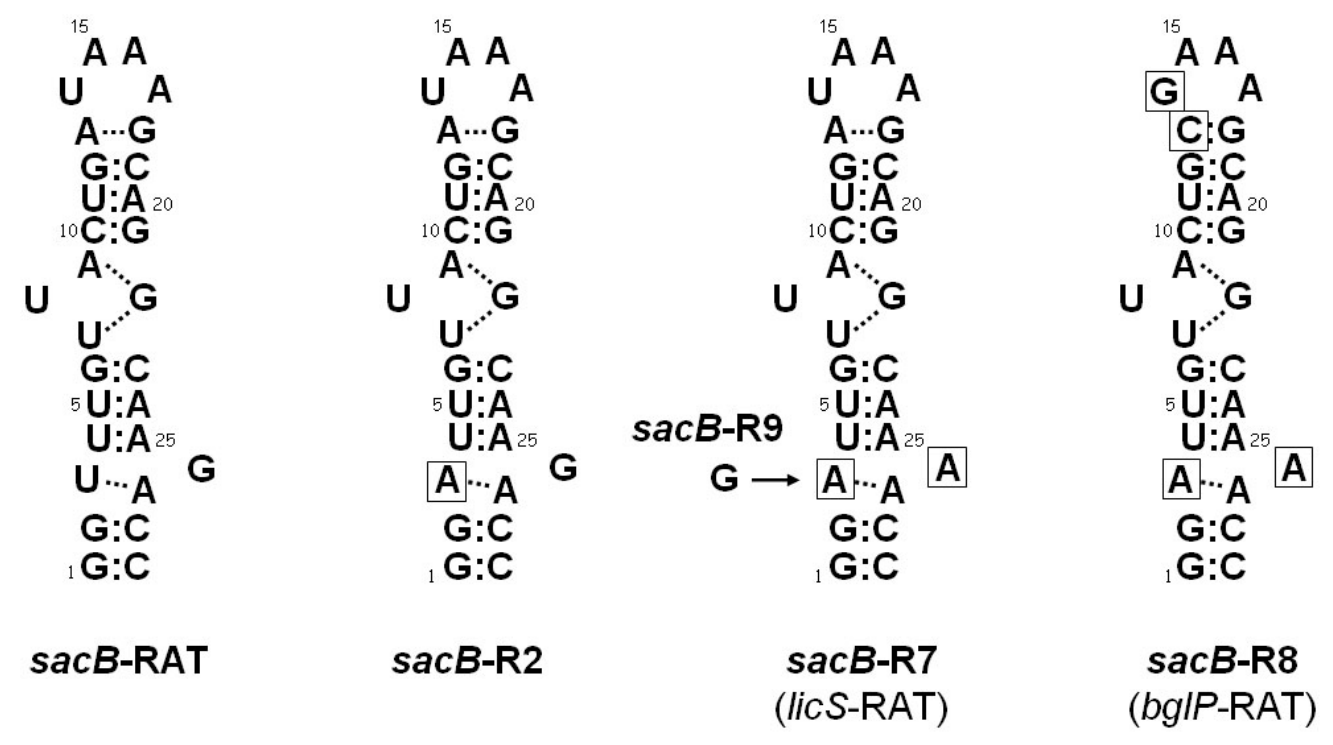

Figure 5.10. Secondary structures of the wild type sacB, sacB-R2, sacB-R7 and sacB-R8 RATs. Bases that differ from the $s a c B$ RAT are boxed. The mutations introduced into the $s a c B$ RAT convert it gradually to the $l i c S$ ( $\operatorname{sacB}-\mathrm{R} 7)$ and the $b g l P$ RATs ( $\operatorname{sac} B-\mathrm{R} 8)$.

The determination of $\beta$-galactosidase regulation conferred by this RAT demonstrated induction not only by sucrose but also by salicin (see Table 12). Induction by salicin was completely lost in a LicT mutant whereas sucrose induction was lost in the sacT mutant strain. Binding of LicT to the $s a c B$-R7 RAT was verified by an electrophoretic mobility shift assay. While the RNA-binding domain of LicT was unable to retard the wild type RAT of $s a c B$, the $s a c B-\mathrm{R} 7 \mathrm{RAT}$ was bound by this protein (see Fig. 5.11). Thus, the A at position 26 is an important feature that makes the RAT a target for LicT. A mutation of $2 \mathrm{bp}$ affecting the top loop converts the $l i c S$-RAT ( $s a c B-\mathrm{R} 7$ ) to the $b g l P$ RAT ( $s a c B-\mathrm{R} 8$ ). These mutations increase the affinity of both LicT and SacT as inferred from $\beta$-galactosidase activities of the $s a c B$-R8lacZ fusion strains (see Table 12). This finding is in good agreement with the observed stronger salicin-dependent induction of the $b g l P H$ operon as compared to the licS gene by the antiterminator LicT (Schnetz et al., 1996). As observed for the sacB-R7 RAT, the sacB-R8 RAT was bound by the RNA-binding domain of LicT in vitro (see Fig. 5.11). The importance of A26 for recognition by LicT is underlined by the analysis of the sacB-R9 RAT in which the $\mathrm{A}$ at position 3 (present in $s a c B-\mathrm{R} 7$ and the cognate targets of LicT) is replaced by a $\mathrm{G}$ (see Fig. 5.10). 


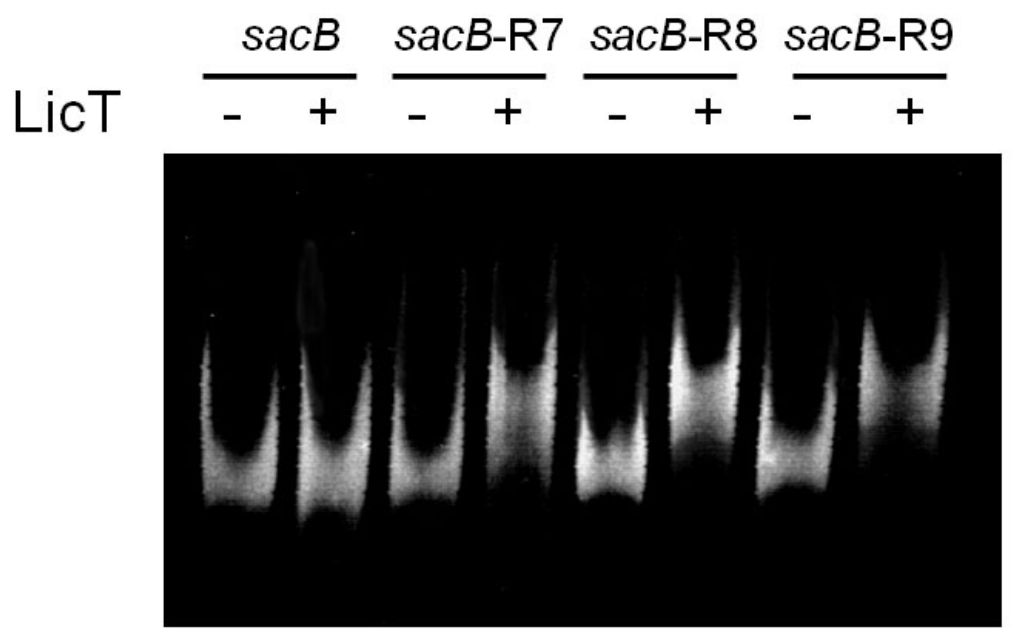

Figure 5.11. Electrophoretic mobility shift analysis of the interaction between the wild type sacB and several mutant RAT RNAs (see Fig. 5.9) with the RNA-binding domain of LicT. In all lanes, 100 pmol of RNA were used. In the lanes labeled with „,+, 250 pmol of LicT were added prior to electrophoresis.

As shown in Table 12, the presence of this RAT allows even higher LicT-dependent induction by salicin. Again, this RAT was recognized by LicT in vitro (see Fig. 5.11). However, this mutation did not affect binding specificity since $s a c B$-R9 was also a target for SacT. Thus, for LicT and SacT, position 3 seems to be most important to maintain the proper RAT structure, whereas the opposing A at position 26 is important for allowing efficient binding by LicT.

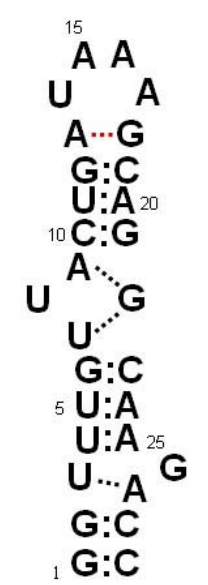

SacB-RAT

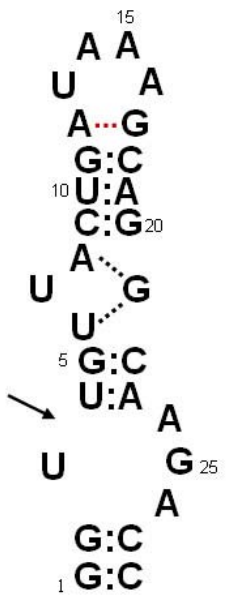

sacB-R10

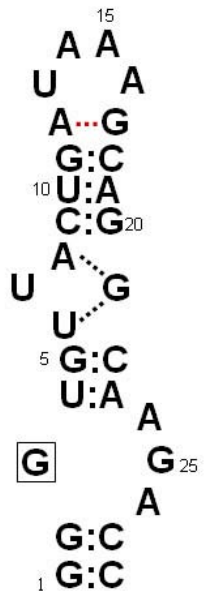

sacB-R11

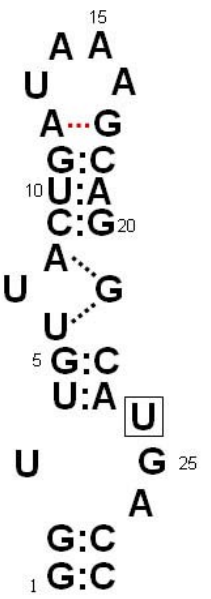

sacB-R12

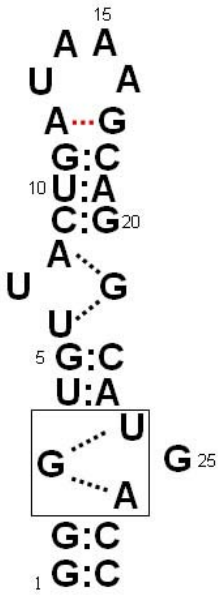

sacB-R13

Figure 5.12. Gradual conversion of the lower loop region of the wild type sacB RAT to a stucture similar to that found in the ptsG RAT. A deletion of a $\mathrm{U}$ in $s a c B-\mathrm{R} 10$ is indicated by an arrow. Bases that differ from $s a c B$-RAT are boxed. The lower loop region in the $s a c B$-R13 mutant is identical to that of the pts $G$ RAT (see Fig. 5.2) 


\section{Discrimination between SacY and GlcT}

The pts $G$ RAT is most different from all other RAT structures in B. subtilis due to the triple base pairing in the lower loop region (see above, Fig. 5.2; Schilling et al., 2004). It has been proposed that this distinct structure rather than the details of the actual nucleotide sequence is important for recognition by GlcT. To verify this assumption we introduced mutations into the $s a c B$ RAT that made its structure gradually more similar to that of the $p t s G$ RAT. In a first step, the $U$ at position 4 was deleted (Fig. 5.12). This mutation is a reversal of the conversion from the $p t s G$ to the $p t s G$-R1 RAT (insertion of one base at position 4, see Fig. 5.3), but in the context of the $s a c B$ RAT. The resulting $s a c B$-R10 RAT allowed a very weak SacT-dependent induction by sucrose. In contrast, this RAT was not at all recognized by GlcT as concluded from the absence of induction by glucose. Here, the U3 might form a base pair with either A24 or A26 thus forming a structure weakly recognized and sufficiently stabilized by SacT to allow antitermination.

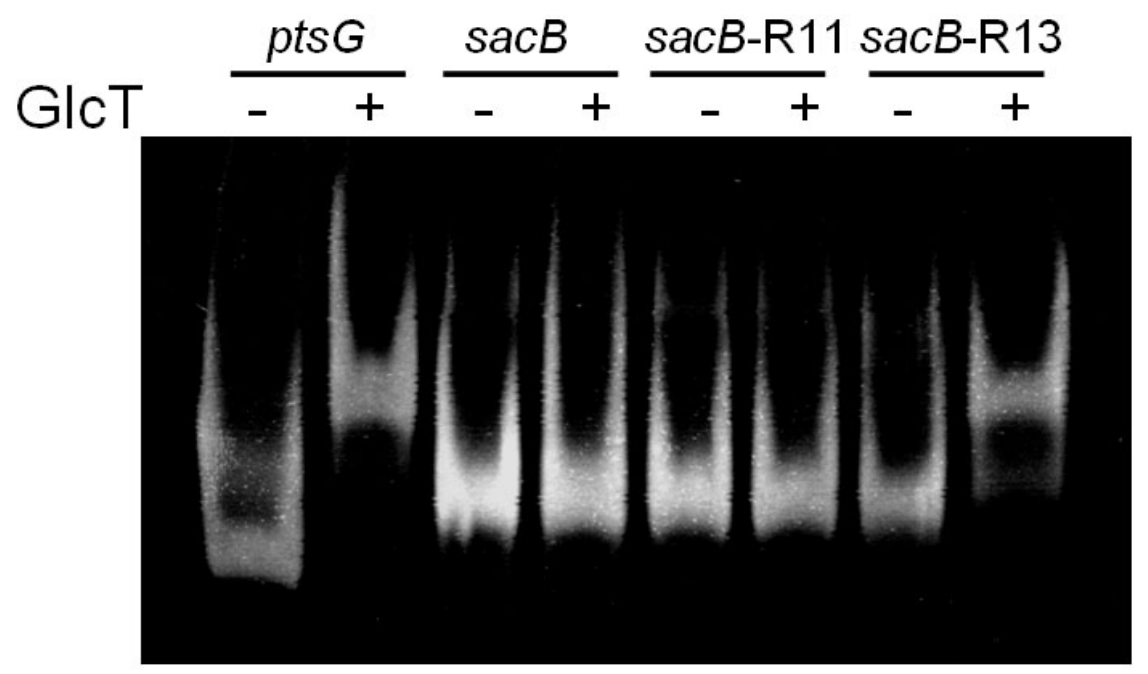

Figure 5.13. Electrophoretic mobility shift analysis of the interaction between the ptsG and sacB RAT RNAs, several sacB mutant RAT RNAs and the RNA-binding domain of GlcT. In all lanes, 100 pmol of RAT RNA were used. In the lanes labeled with „,““, 250 pmol of GlcT were added prior to electrophoresis.

The $s a c B$-R10 mutant RAT was then parent to two further variants. In the $s a c B-\mathrm{R} 11-\mathrm{RAT}$, the $\mathrm{U} 3$ was replaced by a $\mathrm{G}$ (see Fig. 5.12). In the $p t s G \mathrm{RAT}$, a $\mathrm{G}$ at this position contacts the nucleotides at positions 24 (U) and 26 (A). However, this does not seem to be the case with the two adenines in $s a c B-\mathrm{R} 11$. This RAT does not confer induction to the $l a c Z$ gene, however, the read through was somewhat increased even in the absence of any inducer (see Table 12). A replacement of $\mathrm{A} 24$ present in $s a c B-\mathrm{R} 10$ by a $\mathrm{U}$ (as in the ptsG RAT at this position) 
resulted in complete loss of expression of the reporter gene (see Table 12, sacB-R12, see Fig. 5.12). By replacing the $\mathrm{U} 3$ of $s a c B-\mathrm{R} 12$ by a $\mathrm{G}$, we obtained a lower loop that is identical to that found in the ptsG RAT ( $s a c B-\mathrm{R} 13$, Fig. 5.12). Indeed, the $s a c B-\mathrm{R} 13-l a c Z$ fusion was induced by glucose and sucrose (see Table 12). Since salicin and sucrose are known to activate GlcT (Langbein et al., 1999), we tested the expression of this fusion in a licT sacT sacY triple mutant as well as in a glcT mutant strain. As expected, the combined deletion of licT, sacT, and $s a c Y$ did not affect the induction by any of the sugars whereas no induction was observed in the glcT mutant strain (see Table 12). Thus, this RAT is exclusively recognized by GlcT. To verify this observation we performed electrophoretic mobility shift assays in the presence of the RNA-binding domain of GlcT using the RATs of $p t s G$ and $s a c B$ as controls as well as the $s a c B$-R11- and $s a c B$-R13-RATs (see Fig. 5.13). As previously observed, GlcT is capable of binding its cognate ptsG RAT. In contrast, the $s a c B$ RAT was not recognized by GlcT. Similarly, the $s a c B-\mathrm{R} 11-\mathrm{RAT}$ was not retarded. As expected from the transcription regulation conferred by GlcT and the $s a c B$-R13 RAT, an RNA-fragment containing this RAT was bound by the RNA-binding domain of GlcT. These findings confirm the important role of G3, U24, and A26 for the formation of the structure in the lower loop and in GlcT binding.

\section{Discussion}

Several distinct mechanisms contribute to the specificity of the four antitermination systems present in B. subtilis. These include, first, the sugar permeases and their interactions with their substrates and with the cognate antitermination proteins. Second, carbon catabolite repression limits the conditions under which certain systems are expressed and the antiterminator proteins active. Finally, the interaction between the antiterminator proteins and the RAT RNAs makes a major contribution to regulatory specificity.

The sugar permeases of the PTS can transport and phosphorylate only one substrate, or they can exhibit a relaxed specificity, i.e. they may transport more than one sugar. The glucose permease PtsG is known to transport sucrose and salicin in addition to glucose thus explaining the induction of ptsG expression by these sugars (Langbein et al., 1999) (see Table 11). A relaxed specificity has also been observed for the GlcB permease from Staphylococcus carnosus which is also capable of transporting salicin in addition to glucose (Christiansen and Hengstenberg, 1999). Similarly, the $\beta$-glucoside permease BglP is able to transport glucose, although with a low efficiency (see Fig. 5.7). In wild type strains, the $b g l P$ gene is strongly repressed in the presence of glucose, thus, this relaxed specificity has no 
biological consequence. In contrast, the two sucrose permeases seem to be highly specific for sucrose, and the SacX permease is regarded as being inactive since it does not contribute to sucrose transport (Kunst et al., 1974). All experiments with the different antitermination systems published so far did not provide any indication that a permease might interact with a non-cognate antiterminator protein. This might reflect the parallel evolution of the permeases and their targets, the PRD-I domains of the antitermination proteins (Greenberg et al., 2002). Indeed, the control of the antitermination proteins by the corresponding sugar-specific permeases works beyond the species barrier as shown for B. subtilis LicT in E. coli or S. carnosus GlcT in B. subtilis (Knezevic et al., 2000; Schnetz et al., 1996).

Bacteria use carbon sources in a hierarchical order, i.e. those that are most easily metabolized with a maximum yield of energy are preferred. In B. subtilis, glucose is the preferred carbon source, and the presence of glucose prevents the activity of many enzymes as well as the expression of genes and operons that are required for the utilization of alternative carbon sources. Among the genes studied here, only pts $G$ is induced by glucose (via antitermination) whereas $s a c P A, \operatorname{bglPH}$, and $l i c S$ are repressed. This repression is achieved by two independent mechanisms: First, the CcpA repressor protein binds target sites in the promoter regions of these genes and prevents their expression if glucose is present. Second, the antiterminator proteins SacT and LicT, which are required for the expression of these genes, are inactive as long as glucose is present. In the absence of glucose they are phosphorylated at their PRD-II and thereby activated by HPr (Krüger et al., 1996; Lindner et al., 1999; Stülke et al., 1998). These two mechanisms result in the absence of the BglP permease if glucose is available. Only in the glcT mutant strain, if glucose is unable to exert carbon catabolite repression since it can not efficiently be transported into the cell, the $b g l P$ gene can escape carbon catabolite repression and the BglP protein may exert a weak glucose transport activity which is sufficient for the activation of LicT (see Fig. 5.7, Table 11).

A major specificity determinant in transcription regulation by the four antiterminator proteins is the RNA-protein interaction. As shown previously, the loop structures of the RATs are crucial for the specific recognition (Schilling et al., 2004; Yang et al., 2002). In this work, we have identified all the factors that determine the specificity for any of the four antiterminator proteins (see Fig. 5.14).

GlcT is unique in that it requires two identical and nearly symmetrical triple base pairings in the RAT. In contrast, LicT, SacT, and SacY bind RAT structures that resemble the upper triple base pair in the ptsG RAT, but differ significantly in the lower loop. 


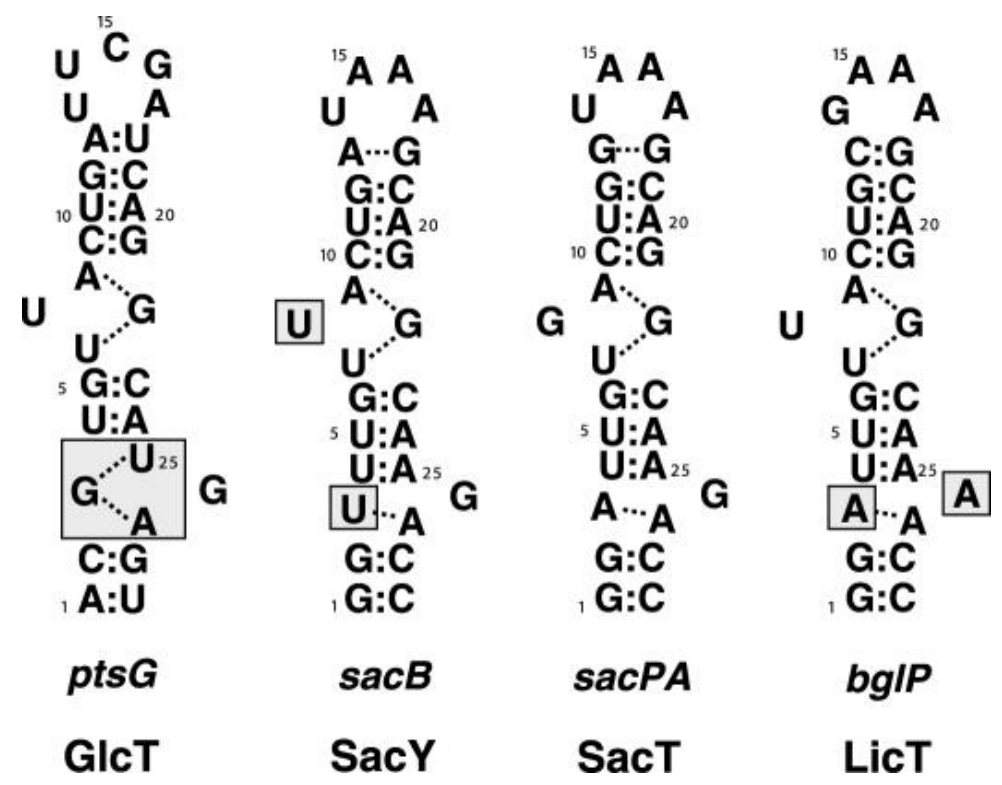

Figure 5.14. Summary of the relevant features that cause protein-RNA recognition specificity of the RAT RNAs of B. subtilis. Boxes indicate nucleotides that switch specificity towards the appropriate antiterminator proteins. For $p t s G$, the structure of the lower loop region discriminates it from all the other RAT-structures and facilitates exclusive GlcT binding. SacY binding depends on the Us found at positions 3 and 8 in the $\operatorname{sacB}$ RAT. No specificity determinant could be found for SacT, as it binds to all RAT structures except for that of $p t s G$. SacT signaling specificity is achieved by the control of the protein's activity by dual PTS-dependent phosphorylation. The LicT targets $(b g l P$, lic $S)$ are characterized by the essential As found at positions 3 and 26.

A mutation that changes the lower loop of the pts $G$ RAT towards that found in the RAT recognized by the other antiterminator proteins ( $p t s G-\mathrm{R} 1, p t s G-\mathrm{R} 5$, see Table 11) prevented recognition by GlcT and allowed binding by SacT and LicT. Similarly, a mutation of the $s a c B$ RAT, which affected the lower loop and allowed the formation of a triple base pair, resulted in loss of SacY and SacT binding whereas GlcT recognized such a structure ( $\operatorname{sacB}-\mathrm{R} 13$, see Figs. 5.12 and 5.13; Table 12).

LicT recognizes structures that are highly similar to the targets of SacT and SacY. An inspection of the RAT structures reveals that the LicT targets are unique in having an A at position 26 (see Fig. 5.2). The importance of this position is underlined by our mutation analysis of the $s a c B$ RAT. The importance of A26 for recognition by LicT is also supported by the structure of the LicT-RAT complex. There are several contacts of LicT with A26 and the sugar phosphate backbone in its immediate neighborhood (Yang et al., 2002). The differential role of guanine and adenine residues for recognition of nucleic acids by proteins is well established (Nobeli et al., 2001). Moreover, the data indicate that the A at position 26 is necessary but not sufficient for LicT binding. In addition, a purine base is required at the opposing position 3 of the RAT. This is in good agreement with a previous study (Aymerich 
and Steinmetz, 1992). Interestingly, a $\mathrm{G}$ at position 3 (sacB-R9) allows much higher LicT-, but also SacT-dependent antitermination as compared to a similar RAT containing an A at this position ( $\operatorname{sacB}-\mathrm{R} 7)$.

There are conflicting reports on the recognition of the $s a c B$ RAT by SacT (Aymerich and Steinmetz, 1992; Manival et al., 1997). We observed that the $\operatorname{sacB}$ RAT is recognized by both SacT and SacY. Interestingly, the SacT-dependent induction of $s a c B$ is stronger than the induction mediated by the cognate antiterminator, SacY. Thus, SacT induces both the $s a c P A$ operon and the $s a c B$ gene encoding levansucrase. In contrast, SacY exerts only a very minor effect at the $s a c P A$ RAT (identical to $s a c B-R 6$ ). Since SacT is active at both high and low sucrose concentrations whereas SacY is active only in the presence of large amounts of sucrose, SacT may be regarded as the major antiterminator protein controlling sucrose utilization. The minor role of SacY is also illustrated by the weak affinity of this protein to the $s a c B$ RAT which is two orders of magnitude lower than the affinities observed for LicT and GlcT with their respective targets (Declerck et al., 1999, I. Langbein and J. Stülke, unpublished data). A step-wise conversion of the $s a c B$ RAT into a $s a c P A$-RAT-like structure revealed the following observations: Single base mutations (see Fig. 5.8, sacB-R2, sacB-R3, $s a c B-\mathrm{R} 4$, Table 12) all enhanced the binding of SacT, whereas the double and triple mutations (see Fig. 5.8, sacB-R5, $s a c B$-R6) had additive effects. Most single mutations and the double mutation prevented SacY binding. However, the triple mutation which did also affect the top loop neutralized the negative effect of the two other nucleotides and restored binding of SacY.

Taken together, our data indicate that SacT is the most promiscuous of the antitermination proteins whereas GlcT at the other end of the spectrum is strictly confined to its cognate $p t s G$ RAT due to its specific structural demands. In the living cell, glucose plays a special role as the by far most preferred carbon source. Therefore, it is advantageous for the bacteria to have a regulatory system for glucose utilization that avoids any risk of cross-talk. On the other hand, SacT induces both sucrose catabolic systems, but it does not mediate antitermination at the bglP RAT in wild type bacteria (Krüger and Hecker, 1995). As shown here and in previous publications, SacT can bind $b g l P$-like RAT structures (Manival et al., 1997) (Table 12). It is so far unknown why SacT does not induce the $b g l P H$ operon in wild type bacteria. More factors such as the sequence context surrounding the RAT, the top loop, and the overall stability of the different RAT/terminator couples may provide additional levels for controlling the effective interaction with the antiterminator proteins. A careful analysis of the data presented here indicates that this is indeed the case. More work will be required to study the contributions of these factors. 


\section{Discussion}

\subsection{Effects of organic acids on the central metabolism of $B$. subtilis}

In its natural habitat, $B$. subtilis faces a highly competitive environment. The bacterium is subjected to rapidly changing conditions, e.g. temperature and nutrient supply. Nevertheless, B. subtilis is also competing with other organisms for the available resources. Some soil organisms avoid direct competition by occupying exclusive ecological niches, others developed ingenious strategies to defend their habitat against intruders, e.g. by the production of antibiotics. In addition, metabolism of the organisms needs to adapt to the above mentioned challenges. The environmental conditions are sensed, and the obtained information is interpreted and transferred into gene and protein regulatory processes.

As B. subtilis is widely used for the industrial production of amylases, proteases, lipases, and vitamins, there is much interest in optimizing the metabolism of industrially used B. subtilis strains towards higher production yields (Harwood, 1992; Sauer et al., 1996). To monitor the effects of different nutrient sources or genomic modifications on a global level, new screening methods have been developed over the last decade. B. subtilis was among the first fully sequenced bacteria (Kunst et al., 1997), which enabled scientists to study this organism on global transcriptomic and proteomic levels (Hecker, 2003; Hecker and Engelmann, 2000; Yoshida et al., 2001). Much effort has been devoted to study the influence of glucose and other sugars on the central metabolism of different eukaryotic and prokaryotic organisms. In this work, the methods of DNA-microarray and fluxome analyses were used to study the influences of the organic acids glutamate and succinate on the central metabolism of glucose grown B. subtilis. The combined evaluation of data derived from these two methods was expected to indicate for so far unknown regulatory contexts.

\section{Metabolic pathways that show accurate correlation between the two global screening methods}

With only few exceptions, the results obtained from the two global screening methods, i.e. microarray analysis and metabolic flux analysis, are in good agreement for most of the studied genes. The transcriptomic data revealed that the expression of most of the glycolytic genes is not affected by the availability of glutamate and succinate. This is also true for the genes coding for the pentose phosphate pathway (see Figs. 2.2 and 6.1). The glycolytic genes coding for enzymes that catalyze reversible reactions are known to be constitutively expressed, and it is therefore no surprise that no significant regulation was observed in the 
transcriptomic analysis. The highest regulatory effect on genes of these two pathways has been observed for the ptsGHI and gapA operon in previous studies (Ludwig et al., 2001; Stülke et al., 1997). Both were highly induced in the presence of glucose, and were not expressed when no sugar was available. The transcription of the gapA operon has also been found to be influenced by the availability of amino acids (Ludwig et al., 2001). Full induction was only observed when the medium was supplemented with casein hydrolysate. However, the addition of glutamate and succinate had no effect on the transcription of this operon in this study. The highest upregulation of glycolytic genes was observed for $p f k A$ and $p y k A$ when glutamate and succinate were present in the medium, but only a weak 1.3-fold induction was observed. $P y k A$ and $p f k A$ are clustered in the same operon. The phosphofructokinase is known to be allosterically controlled by ADP and fructose-6-phosphate in Bacilli (Evans et al., 1981; Yoshida, 1972), but its regulation on a transcriptional or translational level is a controversial issue. On the one hand, it was reported that $p f k A$ is slightly upregulated in the presence of glucose when using $\beta$-galactosidase assays and macroarrays (Blencke et al., 2003; Ludwig et al., 2001). On the other hand, no higher protein amount was detectable in a proteomic study under the same conditions (Tobisch et al., 1999b), but the method of 2D gel electrophoresis, that was used to obtain the proteomic data, is not sensitive enough to detect such a small regulatory effect. In Lactococcus lactis, pfk and pyk are also organized in an operon, i.e. the las operon (Llanos et al., 1993). Transcription of the las operon is activated by the presence of sugars transported via the PTS by means of CcpA (Luesink et al., 1998). In a ccpA mutant strain, the transcription level of this operon is 4-fold reduced. A cre site was found upstream of the promoter region of the las operon, confirming the assumption that CcpA induces this operon when PTS sugars are available. Also, in L. casei pfk and pyk are clustered in an operon. However, in a $c c p A$ mutant the transcription of these genes is slightly increased, indicating a PTS/CcpA dependent regulation mode that differs from that of L. lactis (Viana et al., 2005). Similar to the situation in B. subtilis, the mode of $p f k A$ regulation is not clear in L. casei. Data obtained from microarray analysis alone are not accurate enough to suggest a significant upregulation with the observed 1.3-fold change for $p f k A$. Due to the extensive tests that were carried out prior to this study, all transcription changes above 1.5-fold are considered as significant.

But the transcriptomic data can only give ratios for the gene expression under the two studied conditions. They can neither give information about the absolute expression rate of the analyzed genes, nor do they reflect the activity of the respective enzymes. The $\beta$-galactosidase assays that were carried out in this work can also not reflect the activity of a 
certain enzyme, but in addition to microarray analysis, it can give indication if a gene is highly expressed or not, even if the ratio for the gene examined under the two conditions is 1 . The $\beta$-galactosidase assays revealed that $p t s G$ and the gap $A$ operon are highly expressed under both conditions studied here. This high expression is caused by the presence of glucose under both conditions and is also in good agreement with previous findings (Ludwig et al., 2001; Stülke et al., 1997). It was also reported, that casein hydrolysate has a positive effect on the transcription of this operon (Ludwig et al., 2001). Thus, glucose and amino acids seem to have a synergistic effect on the expression of the gapA operon. However, no additional effect upon addition of glutamate and succinate was observed in the microarray analysis.

Contrary to the constant expression of the glycolytic genes, the flux into glycolysis is slightly decreased in the presence of organic acids. The reason for that is a reduced flux from glucose-6-phosphate to fructose-6-phosphate, carried out by the glucose-6-phosphate isomerase (Pgi), in the presence of glutamate and succinate. This can be explained with the higher portion of glucose-6-phosphate entering the pentose phosphate pathway via glucose-6phosphate dehydrogenase (Zwf) under this condition. For both enzymes, glucose-6-phosphate is the direct substrate. With the used methods, it can not be distinguished whether a lower enzymatic activity of glucose-6-phosphate isomerase or a higher activity of glucose-6phosphate dehydrogenase is the reason for the change in metabolic fluxes. The transcription of both genes is not altered under any of the analyzed conditions. It was reported in previous studies that the expression of these genes is also not affected by the presence or absence of glucose (Blencke et al., 2003; Ludwig et al., 2001). In E. coli, it was shown that the deletion of pgi resulted in a significant higher flux of glucose-6-phosphate into the PPP when grown on glucose. At the same time, the maximum growth rate was greatly reduced (Canonaco et $a l ., 2001)$. The impact on growth rate could be traced back to a disturbed reducing power balance due to the difficulty of $E$. coli metabolism to reoxidize NADPH in the pgi mutant. When grown on fructose, which does not need the glucose-6-phosphate isomerase to enter glycolysis, the pgi mutant grew with maximum specific growth rate (Canonaco et al., 2001). This demonstrates that a reduced activity of glucose-6-phosphate isomerase could indeed lead to a higher flux of glucose-6-phosphate into the PPP. However, an enzyme catalyzing a downstream reaction could also lead to the observed metabolic fluxes. A good candidate gene would be $y w l F$, coding for ribulose-5-phosphate epimerase. 


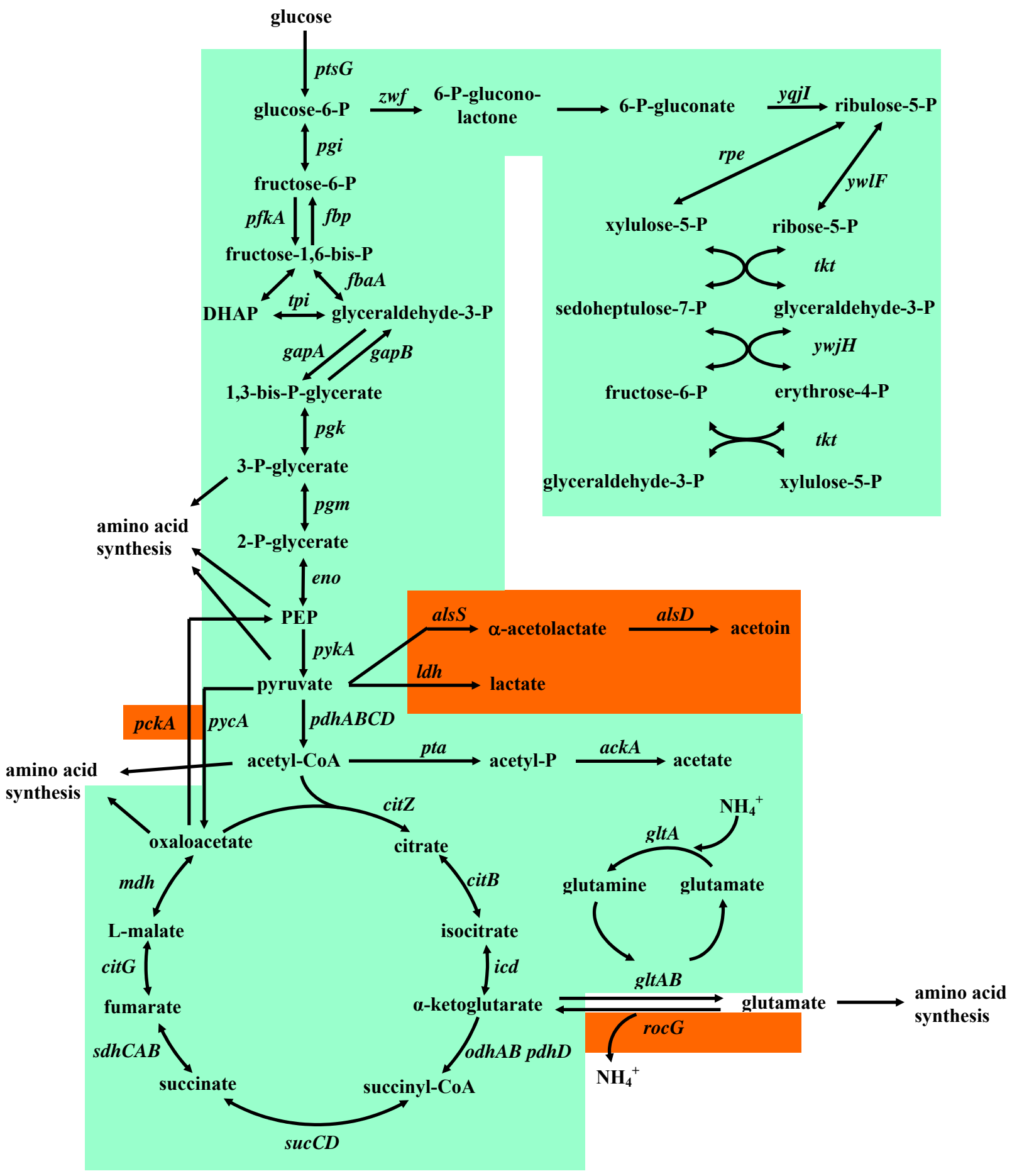

Figure 6.1 Overview on the genes of central metabolic pathways in B. subtilis.

Metabolic pathways that showed good correlation for the used screening methods are indicated in green. Pathways that showed no correlation between the used methods are indicated in red. 
The transcriptomic analysis revealed a weak induction of $y w l F$ in the presence of organic acids (1.45-fold), and this could be a possible reason for the increased flux into the pentose phosphate pathway. It is interesting to note that the flux into the PPP was relatively high under both tested conditions. In a previous study, it was reported that the metabolic flux into the PPP increases with the growth rate of B. subtilis (Dauner and Sauer, 2001). Also, a relatively high flux into this pathway, which is between 30 and 50 percent of the metabolized glucose, has been observed (Sauer et al., 1997; Sauer et al., 1996). Therefore, the pentose phosphate pathway can be considered as one of the major pathways for glucose catabolism besides glycolysis. At higher growth rate, and therefore also higher biomass production, $B$. subtilis would have to increase the flux into the PPP to meet its higher demand of NADPH for anabolic reactions. In agreement with this theory, the growth rate was increased by $27 \%$ when glutamate and succinate were available, and the data gained from the fluxome analysis confirmed that a higher portion of the metabolic intermediates is used for biomass generation.

The TCA cycle shows a strong repression in the presence of organic acids. The transcriptomic data revealed a 5- and 6-fold repression of $c i t Z$ and citB, respectively (see Fig. 2.2). These genes code for enzymes that catalyze the initial reactions of the TCA cycle. This significant repression was observed not only on the transcriptomic level, but also the fluxome data revealed a 5-fold lower flux of acetyl-CoA into the TCA cycle when glutamate and succinate were available (see Fig. 2.1). Moreover, the reporter gene analysis of a citB-lacZ fusion showed a 9-fold reduced expression upon addition of these organic compounds. The observed repression is a synergistic effect coming from both glucose and glutamate. It was shown previously, that glucose alone causes a repression of citZ and citB. This repression is increased when glucose and amino acids are present simultaneously (Rosenkrantz et al., 1985; Sonenshein, 2002). Similarly to the synergistic induction of the gapA operon by glucose and amino acids, the TCA cycle is synergistically repressed by the same compounds (Blencke et al., 2003; Blencke et al., 2006; Ludwig et al., 2001). Since the TCA cycle has a dual role, i.e. the catabolism of carbon sources and the anabolism of amino acids, it makes sense that its regulation is affected from both directions (see also chapter 1.1.2).

The regulation mode of $c i t Z$ and $c i t B$ might not be the only reason for the reduced flux from acetyl-CoA through the TCA cycle. A significant increase of acetate production due to an induced transcription of the respective enzymes, phosphotransacetylase (Pta) and acetate kinase (AckA) lowers the availability of acetyl-CoA for the TCA cycle and therefore leads to a "by-pass" of this pathway. It was shown in a pta deletion mutant that citB expression is 2-fold increased, and when overexpressing phosphotransacetylase, citB is even repressed 
when no PTS sugar but gluconate is available (Blencke et al., 2006). This indicates that the flux from acetyl-CoA to acetate has indeed an influence on the regulation of the TCA cycle.

High amounts of acetate are already produced without the addition of glutamate and succinate, since pta and ackA are induced in the presence of glucose by the means of CcpA (Grundy et al., 1993; Presecan-Siedel et al., 1999; Turinsky et al., 1998). Similar as observed for the TCA cycle, the presence of glutamate and succinate has a synergistic effect on gene transcription, but now in the opposite direction. AckA was found to be 3 -fold induced and the flux from acetyl-CoA to acetate was significant higher in the presence of glutamate and succinate. It was recently found that $a c k A$, in addition to CcpA, is also regulated by the transcriptional regulator CodY (Molle et al., 2003; Shivers et al., 2006). The activity of CodY is regulated by the intracellular levels of GTP and branched-chain amino acids (BCAA), i.e. leucine, isoleucine, and valine, which are synthesized from pyruvate. CodY is the repressor of more than 100 genes (Molle et al., 2003). However, ackA represents the first gene that is positively regulated by CodY and this positive effect is exclusively dependent on the availability of BCAA (Shivers et al., 2006). It is known that CcpA and CodY are both involved in the repression of the TCA cycle and genes of BCAA biosynthesis (Kim et al., 2003a; Kim et al., 2002; Kim et al., 2003b; Ludwig et al., 2002a; Shivers and Sonenshein, 2004; Shivers and Sonenshein, 2005; Tojo et al., 2005). The fact that both regulators are also involved in the regulation of ackA suggests that $\operatorname{CodY}$ plays also a significant role in controlling cellular carbon flow. It was found that CcpA and CodY have an additive effect and both regulators are necessary for full activation of ackA expression (Shivers et al., 2006). This would fit very well to the regulatory pattern observed in this study. However, it remains unclear how the presence of glutamate and succinate could lead to the activation of CodY. Site directed mutageneses of the ackA promoter region, affecting CodY binding, could indicate whether CodY is involved in the observed induction of ack $A$ or not.

The transcription of TCA cycle genes other than $c i t Z$ and $c i t B$ is not severely affected by the availability of glutamate and succinate. This reflects the need of the respective enzymes under both studied conditions. The fluxome analysis revealed that a high portion of the external glutamate is converted to 2-oxoglutarate and thus entering the TCA cycle (see Fig. 2.1). Also external succinate feeds into this pathway. Under these conditions, the TCA cycle does only seem to fulfill a biosynthetic function. This behavior of the TCA cycle in excess of nutrient sources was also observed for Saccharomyces cerevisiae. The performance of the catabolic and anabolic function was found to be related to the glucose uptake rate and extracellular pH (Blank and Sauer, 2004). 


\section{More than just the sum of its individual parts: Data that show no correlation between the two global screening methods}

The comparison of the data derived from transcriptome and fluxome analyses revealed that in some cases, there is no close correlation for the two global screening methods. The genes coding for acetolactate synthase and acetolactate decarboxylase, converting pyruvate to $\alpha$-acetolactate and acetoin (Cruz Ramos et al., 2000), are highly induced without a notable change in flux to acetoin in the presence of organic acids. In fact, these metabolites were undetectable under any of the studied conditions (see Figs. 2.1 and 6.1). The transcription of $p c k A$ did not change, while a notable change in flux from oxaloacetate to pyruvate occurred. The ratio found by microarray analysis was close to 1 , but the conversion from oxaloacetate to PEP was threefold increased. For $l d h$, a change towards the same direction was measured for the transcriptome and fluxome analyses, but the fold change of 1.7 does not meet the observed 25-fold increase in metabolic fluxes from pyruvate to lactate when glutamate and succinate are present (see Figs. 2.1, 2.2, and 6.1).

These observations do not necessarily mean that the data obtained by any of these two methods is wrong. For a better interpretation of the information, it is important to know the limitations of the two methods. Microarray analysis gives only information about the presence of a certain transcript under a certain condition. But it does not give information for the absolute amount of a transcript. In fact, only the ratio in relation to another condition is obtained (Harrington et al., 2000). For the analysis, it is also assumed that the amount of a certain transcript reflects the amount of the respective protein. But this does not necessarily need to be the case. Due to alternative splicing, a single mRNA can code for several different proteins in eukaryotic cells (Black, 2000). Although this is not the case in prokaryotic organisms, it can not be considered that the amount of transcripts found in a cell completely reflects the amount of the corresponding proteins. A transcribed mRNA can interact with several factors that regulate its translation. These factors can stimulate or inhibit translation initiation (see also chapter 1.2). Also the complete protein synthesis machinery can interact differently with the same mRNA under varied conditions (Laursen et al., 2005). Protein activity can also be modulated by modifications, e.g. phosphorylation events, and nevertheless, the half-life of a protein can also be altered under different environmental conditions (Jenal and Hengge-Aronis, 2003). Therefore, the transcriptome can only partly reflect the regulatory processes of an organism.

A way to determine the activity of metabolic enzymes is the combination of transcriptome analysis with metabolic flux analysis. Metabolic flux analysis is a method to 
determine the proportion that each metabolic pathway exerts when an organism is metabolizing a given substrate under a certain condition (Christensen et al., 2002; Nielsen, 2003; Sauer, 2004). However, this method also has some drawbacks. Metabolic pathways that are not coupled to measurable fluxes can not be resolved (Wiechert, 2001). Also in case of "parallel metabolic pathways", where none of the branches is coupled to a measurable variable, it is impossible to resolve the "two branch fluxes" (Sonntag et al., 1993; Wiechert, 2001). Moreover, the whole method is based on a computer model of the studied organism. Therefore, the data obtained with this method can only be as accurate as the underlying model (Covert et al., 2001; Wiechert, 2001).

Both methods are established and already extensively used to analyze the metabolism of B. subtilis (Blencke et al., 2003; Dauner et al., 2001; Koburger et al., 2005; Moreno et al., 2001; Sauer et al., 1996). The combination of data sets from both methods can indicate so far unknown regulatory processes on the protein level. This could be the case for the above mentioned genes. The regulation of the alsSD operon and $l d h$ are emphasized in this discussion, since they represent both extremes observed in this study, i.e. a high induction of transcription without changed enzymatic activity, and a significant higher enzymatic activity at almost unchanged transcription rate.

The alsSD operon was found to be 10 -fold induced in the presence of organic acids. However, no acetoin formation was detectable under both studied conditions (see Figs. 2.1 and 2.2). It was reported that detectable amounts of acetoin are only produced when $B$. subtilis is in the stationary phase. Interestingly, a 10-fold induction was also observed when the bacteria were in stationary phase (Renna et al., 1993). The alsSD operon is controlled by the transcription activator AlsR (Cruz Ramos et al., 2000; Renna et al., 1993). As mentioned above, high amounts of acetate are produced when the bacterium grows on glucose, and it was reported that either acetate or a lowered $\mathrm{pH}$ value activates AlsR (Holtzclaw and Chapman, 1975; Renna et al., 1993). There is also evidence that the alsSD operon is indirectly induced by the redox regulator Fnr under anaerobic conditions (Cruz Ramos et al., 2000). Additionally, CcpA plays an indirect role in the induction of this operon in the presence of high amounts of glucose (Renna et al., 1993; Tobisch et al., 1999b). The data indicate that no acetoin is formed although the alsSD operon is highly induced upon addition of glutamate and succinate. Since glucose is present under both conditions, it can also be assumed that the operon is already induced without the addition of organic acids. The absence of the respective product under both conditions might indicate an additional regulatory level. On the one hand, translation initiation could be controlled by an additional factor, e.g. a 
riboswitch, ncRNA, or a protein factor. On the other hand, activity of acetolactate synthase and/or acetolactate decarboxylase might be controlled by allosteric inhibitors or by protein modification. Indeed, the phosphorylation of acetolactate decarboxylase was recently reported (Lévine et al., 2006). The trigger might be the intracellular acetate concentration, since the $\mathrm{pH}$ value of the medium was constant and no oxygen limitations occurred.

The transcription of the $l d h$ gene, encoding lactate dehydrogenase, was 1.7-fold induced when glutamate and succinate were present. Although no lactate was detectable before, significant amounts of lactate were produced upon addition of organic acids. On the other hand, the flux from pyruvate to acetyl-CoA was not affected by the higher activity of lactate dehydrogenase, which also uses pyruvate as its direct substrate (see Figs. 2.1, 2.2, 6.1). It was demonstrated in $E$. coli that overexpression of $l d h$ did also not alter the flux from pyruvate to acetyl-CoA, and when the organism was grown under the same conditions, a higher level of lactate dehydrogenase did also not automatically result in higher lactate production (Yang et al., 1999). This implies that the amount of lactate dehydrogenase is not a limiting factor for the respective enzymatic reaction, and the enzyme might be controlled on the protein level. In addition, a significant higher amount of acetate production was observed when organic acids were present (see above). By producing acetate, B. subtilis yields additional ATP, but more $\mathrm{NAD}^{+}$is reduced to $\mathrm{NADH}$ as well. Since much higher acetate production was observed in the presence of organic acids, the lactate production might be necessary to maintain the redox balance since $\mathrm{NAD}^{+}$is regenerated in this reaction. It was shown for Lactococcus lactis that a functional lactate dehydrogenase is essential for the regeneration of $\mathrm{NAD}^{+}$under aerobic and anaerobic conditions (Neves et al., 2002). Moreover, it was observed that the redox state of B. subtilis controls $l d h$ gene expression in the presence of nitrate (Reents et al., 2006). This supports the idea that the redox state of the cell might be responsible for the higher activity of the lactate dehydrogenase in the presence of organic acids. The increased flux from pyruvate to lactate when glutamate and succinate are present (see above) is paralleled by a reduced flux from pyruvate to oxaloacetate, which is catalyzed by the pyruvate carboxylase (see Fig 2.1). This reciprocal ratio of these two fluxes explains why the flux from pyruvate to acetyl-CoA is not altered under both tested conditions. 


\section{To be or not to be...: Metabolic adaption to a highly competitive environment}

From a global point of view, a general strategy becomes visible. In excess of glucose, glycolysis is highly induced whereas the flux of acetyl-CoA is directed to the overflow metabolic pathways rather than to the TCA cycle. This regulation pattern is intensified upon addition of glutamate and succinate. The TCA cycle is almost exclusively used for anabolism of amino acids and glycolysis, apart from delivering precursors for amico acid synthesis, provides the energy for the cell. The PPP delivers NADPH and building blocks for cell wall and nucleotide synthesis, but the relatively high flux of glucose-6-phosphate might exceed the necessary amount for the supply of these compounds. Thus, the PPP might be also a major pathway for glucose catabolism.

Although this regulation pattern seems to be reasonable, it is noteworthy that the bacterium wastes much energy with this strategy. Normally, bacteria are noted for their efficiency. If the metabolism of $B$. subtilis is not oxygen-limited, it could yield much more ATP per mole glucose when using the TCA cycle for catabolism of acetyl-CoA and oxidative phosphorylation for $\mathrm{NAD}^{+}$recovery instead of using fermentation pathways. To see the benefit of its metabolic strategy, the natural habitat of B. subtilis has to be considered. The bacterium lives in a very competitive environment and struggles with many other organisms for the available nutrient resources. All organisms have to make a tradeoff between rate and yield of ATP production (Pfeiffer et al., 2001; Stucki, 1980). In general, single cellular microorganisms benefit from a higher ATP production rate when they have to share resources with other organisms. This is true for both eukaryotes and prokaryotes. Lactobacilli and Fungi, like Saccharomyces, use fermentation for ATP production even in the presence of oxygen (Poolman, 1993; van Dijken et al., 1993). The result is a rapid exhaustion of the given resources, and the aim of these organisms is to get the most from the dish. This strategy can be seen as a selfish contest in biomass production. However, when the glucose uptake rate becomes limited, Saccharomyces increases respiration and catabolic TCA cycle activity (Blank and Sauer, 2004).

In multicellular organisms, the cells learned to cooperate with each other and to share the available resources. Since they are mostly metabolizing internal resources, they are also not in direct competition with other organisms for nutrient sources. As a result, they aim for a high yield of ATP production rather than a high rate (Pfeiffer et al., 2001). In consequence, the metabolism of $B$. subtilis is optimized for a high production rate and therefore is well adapted to a highly competitive environment. 


\subsection{Specificity in gene regulatory systems}

Although the specificity determinants of the related RAT-antiterminator couples were in the focus of this study, it is obvious that the interplay of several mechanisms results in the specific activation of the relevant systems. This starts already with the more or less specific interaction of the permeases with their appropriate substrate. However, relaxed specificity was found for BglP in this study and was previously reported for PtsG (Langbein et al., 1999). In addition to transcription control by antitermination, the expression of the permeases, except for PtsG and SacX, is also under the control of carbon catabolite repression. This helps to prevent the expression of any other permeases than PtsG and SacX in the presence of glucose. Finally, the negative and positive regulation of the antiterminator proteins at PRD-I and PRD-II, respectively, also helps to keep signals straight in transcriptional antitermination (see also chapter 1.3.3). PRD-I directly interacts with the appropriate permease. The interaction of the permeases with their corresponding antiterminator protein is very specific and no crosstalk was reported so far. On the other hand, it has been reported that HPr, which normally phosphorylates PRD-II, can phosphorylate the PRD-I of LicT in vitro. However, this effect was led back to the non-physiologically high HPr concentrations used in that study (Lindner et al., 1999; Tortosa et al., 2001). Furthermore, high SacT concentration resulted in dimerization and RAT-binding of SacT in vitro independent from its phosphorylation state (Arnaud et al., 1996). Both observations indicate that specificity is also a matter of the concentration of the potential interaction partners.

As described here, a biological gene regulatory system is composed of many different elements that have to interact specifically with each other. An external or internal signal has to be sensed and the gained information is directly or indirectly transmitted to a regulator. The RNA thermometers seem to be the most basic regulatory systems in biological organisms (Narberhaus, 2002; Narberhaus et al., 2006). A certain sequence in the 5'-UTR of an mRNA can act as both sensor and regulator. The only needed interactions are RNA-intramolecular base pairings. This is also true for metabolite dependent riboswitches, but additionally, the RNA has to interact specifically with the sensed molecule.

As soon as a regulatory protein gets involved, the complexity of a regulatory system increases by a considerable amount. This increases also the necessity for specific interactions to prevent any cross-talk with other regulatory systems in the cell. The proteins of the four antiterminator systems interact specifically with their cognate RAT and with two other protein factors, i.e. the respective sugar permease and HPr. The RNA binding domains of the four 
related antiterminator proteins seem to recognize both structure and sequence of their target. However, the structure seems to be more relevant for the specific target recognition.

In contrast, TRAP is a good example of an antiterminator protein that exclusively seems to recognize the sequence rather than the structure of its target RNA (see chapter 1.2.3.4). This protein is composed of 11 identical subunits and controls expression of the $\operatorname{trpEDCFBA}$ operon and $\operatorname{trp} G$ (Antson et al., 1995; Babitzke, 2004; Gollnick, 1994). TRAP forms a donut-like structure and the target RNA is wrapped around the protein ring. It was shown that 11 subunits interact specifically with $11(\mathrm{G} / \mathrm{U}) \mathrm{AG}$ repeats in the RNA target sequence, each repeat separated by two or three nucleotide spacers (Babitzke et al., 1996; Baumann et al., 1997; Yang et al., 1997). In addition to the target RNA sequence, TRAP binds to free tryptophan and to a protein called anti-TRAP. Both ligands modulate TRAP activity.

The fact that most regulatory proteins are made up of distinct subunits, whereas each subunit has a dedicated function and interaction specificity, facilitates the adoption of existing proteins to other functions and thus the evolution of new regulatory systems. The overall composition of LevR, controlling levDEFGsacC operon, and LicR, controlling licBCAH operon, is very similar to that of the four PTS controlled antiterminator proteins (MartinVerstraete et al., 1998; Martin-Verstraete et al., 1990; Stülke et al., 1995; Tobisch et al., 1999a). Likewise, they have two C-terminal PRDs that can be phosphorylated by components of the PTS system or sugar permeases. However, they feature an N-terminal DNA binding domain instead of an RNA binding domain. Thus, they are transcription factors rather than antiterminator proteins. This shows how the exchange of a single subunit could alter the functionality of a regulatory protein.

In contrast to RNA, double stranded DNA does not form complex secondary structures. For a specific interaction with a certain target sequence, a regulatory protein can only interact with the side chains and the edges of the base pairs by hydrogen bonding interactions. The distance of the protein interacting base pairs, the minor and major groove, and the DNA bending and torque are used as specificity determinants (Pabo and Sauer, 1984). The helix-turn-helix motive is the most common DNA binding motive, and DNA binding proteins bind their target mostly as symmetric dimers. The LysR family of transrciptional regulators is a good example how more than 50 autoregulatory transcriptional regulators, each binding to different DNA sequences and regulating the target genes by transcription activation or repression, can evolve from a distant ancestor. The activity of these transcription regulators is modulated by direct interaction with lower molecular weight factors (Schell, 1993). 
Two-component systems make specific signal transduction even more crucial. At least two proteins, a sensor kinase and a response regulator, lead to the specific activation of a target gene mostly based on an external signal. The signal is always transmitted by phosphate group transfer among the proteins of the regulatory system (Albright et al., 1989; Goudreau and Stock, 1998; Hoch and Varughese, 2001). Bacteria with a large genome, such as E. coli and $B$. subtilis, possess up to 40 pairs of two component systems, each dedicated to individual signals and genes. Nostoc punctiformis was found to have even 145 sensor kinases and 103 response regulators (Fabret et al., 1999; Mizuno, 1997). The signaling pathways of chemotaxis or sporulation increase the amount of needed sensor and signaling proteins by a considerable amount and specific protein-protein interaction as well as the concentration of the involved proteins are critical to keep signal transduction straight within the regulatory system (Burbulys et al., 1991; Levit et al., 1998; Trach et al., 1991).

\section{Specificity determinants of the PTS-controlled antiterminator systems}

RNA can form complex secondary and tertiary structures similar to that of proteins (see chapter 1.2.1). Because of the possibility of alternative intramolecular base pairings and the resulting structural complexity of even small RNAs, the exact folding of the RATs as well as the RNA bases involved in RAT-antiterminator interactions are not predictable. Besides NMR and X-ray structural analysis, single nucleotide exchanges can give an indication for secondary structure and specificity determinants. The exchange of nucleotides essential for RNA-protein interaction or secondary structure formation might affect the functionality of the antiterminator system.

The overall secondary structure of the RATs can be subdivided into a top loop and upper and lower loop regions. Furthermore, there is an upper, middle, and lower stem region (see Fig. 6.2). Not only the basic structures of $p t s G, s a c B$, $s a c P A$, licS, and $b g l P$ RAT are very similar, but GlcT, LicT, SacT, and SacY are also recognizing structures that are very similar in sequence (see Fig. 6.3). Therefore, it is not obvious how these proteins can distinguish their specific target from other RATs. The major differences among these sequences can be found in the top loop and lower loop regions (see Fig. 6.3). Hence, it is very likely that the loop regions are involved in specificity determination.

It was found in previous studies that bases located in loop regions are often involved in RNA-protein recognition, whereas bases in stem regions are responsible for stability and structure formation (Nagai, 1996). 


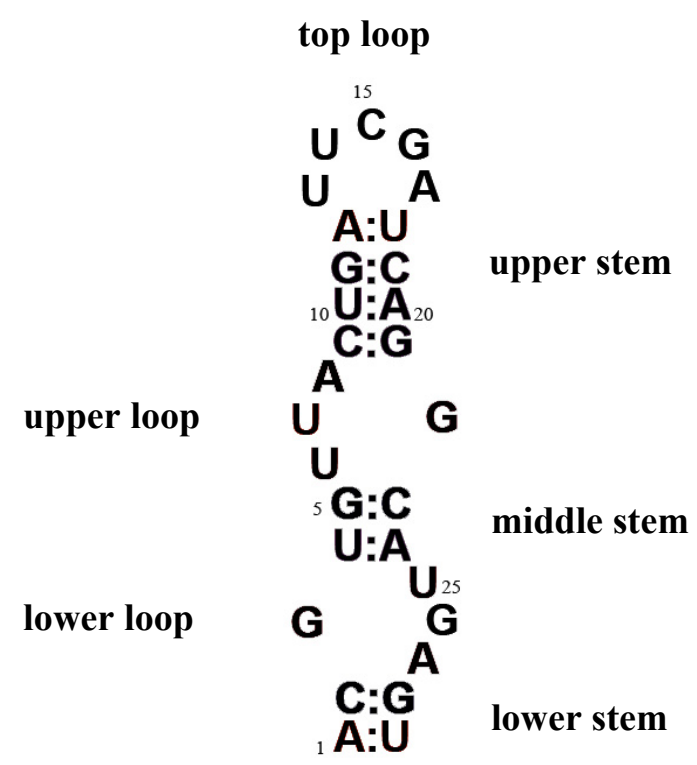

Figure 6.2. Overall secondary structure of the related RATs at the basis of the ptsG RAT (Yang et al., 2002). All RATs share the same basic structure, which can be subdivided into three loop regions (top loop, upper and lower loop) and three stem regions (upper, middle, and lower stem).

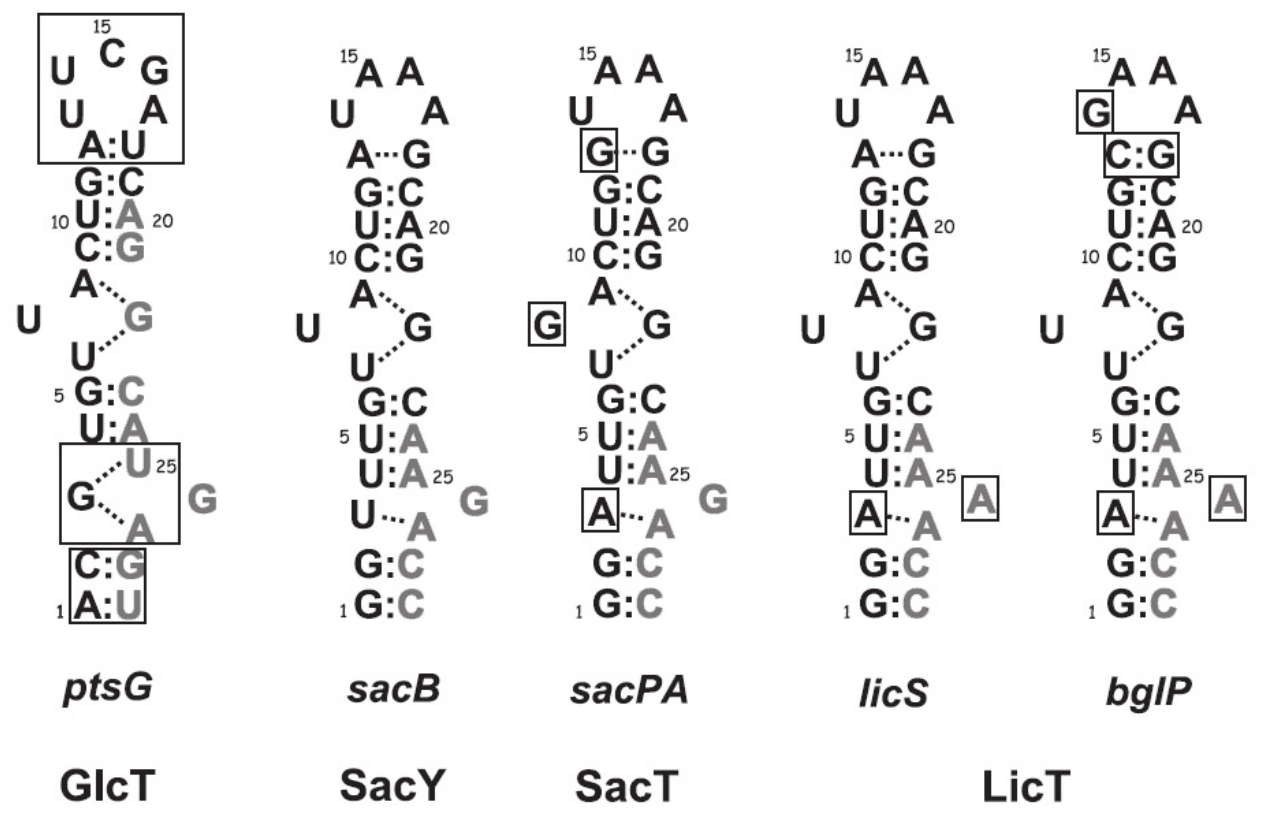

Figure 6.3. Comparison of the secondary structures of the related RAT RNAs of B. subtilis (Schilling et al., 2004; Yang et al., 2002). The relevant antiterminator proteins are indicated below their cognate RAT structures. Boxes indicate nucleotides that differ from the $s a c B$ RAT. Dashed lines indicate bases that are proposed to be in direct contact to each other. Gray nucleotides are overlapping with the terminator structure. The main differences are located in the top loop and lower loop regions. The pts $G$ RAT holds an outstanding position due to its unique lower stem loop and the triple base pairing in the lower loop region. 
The N-peptide of phage $\lambda$ and the MS2 coat protein of phage R17 recognize the top loop structure of their respective target RNA for binding (Grahn et al., 1999; Legault et al., 1998; Valegård et al., 1997). In contrast, site directed mutagenesis of the $s a c B$ and $p t s G$ top loop regions had no effect on target recognition or specificity (Aymerich and Steinmetz, 1992; this work). As the upper loop regions of most of the related RAT structures are identical (except for $b g l P$ and ptsG RAT, see Fig 6.3), it seems to be reasonable that the upper loop cannot contribute to specificity determination.

When analyzing the NMR structure of the LicT-bglP RAT complex, it turned out that the top loop region is indeed not involved in RNA-protein interaction, whereas the upper and lower loop regions of $b g l P$ RAT are both bound by LicT (Yang et al., 2002) (see Figs. 6.4 and 6.5). As the upper loop regions (with the exception of G8 in sacPA RAT, see Fig. 6.3) are also identical in the related RAT structures, it seems unlikely that it can contribute to the specificity of the RAT-protein interaction.

In contrast to the top loop and upper loop regions, the lower loop features more diversity among the RAT sequences (see Fig. 6.3). Accordingly, the site directed mutagenesis of $p t s G$ and $s a c B$ RAT revealed that the specificity determinants of the related RNA-protein couples are indeed located in the lower loop region of the respective RAT structure. The adenines at position 3 and 26 are essential for RAT recognition by LicT. SacT has a relaxed specificity as it binds to all $s a c B$ derived RAT mutants. The data indicate that SacT, aside from binding to its cognate RAT, i.e. $s a c P A$, is also able to bind $s a c B, b g l P$, and licS RAT. However, SacT dependent transcriptional antitermination of $b g l P$ or licS RAT has not been reported in the presence of sucrose. The sacPA RAT seems to have the optimal configuration for SacT binding, as it is bound by SacT with the highest efficiency of all tested RAT sequences. SacY, apart from binding $s a c X$ and $s a c B$ RAT, also binds to the $s a c P A$ RAT sequence. In contrast to $\mathrm{SacT}$, SacY has a more stringent specificity since it does not tolerate the exchange of any of the bases that differ in $s a c B$ and $s a c P A$. These bases are located at positions 3, 8, and 13 in the respective RAT structure (see Fig. 6.3). Only the strict combination of these bases, as found in $\operatorname{sacB}$ (U3, U8, A13) or sacPA RAT (A3, G8, G13), is recognized by SacY (see Fig. 6.3). A single nucleotide exchange in these combinations is not tolerated by SacY.

According to the NMR structure of the LicT-bglP RAT complex, some bases of the middle stem loop are directly interacting with certain amino acids of LicT. Additionally, the protein is directly interacting with the sugar phosphate backbone of the nucleotides of the middle stem loop (see Fig. 6.4). 


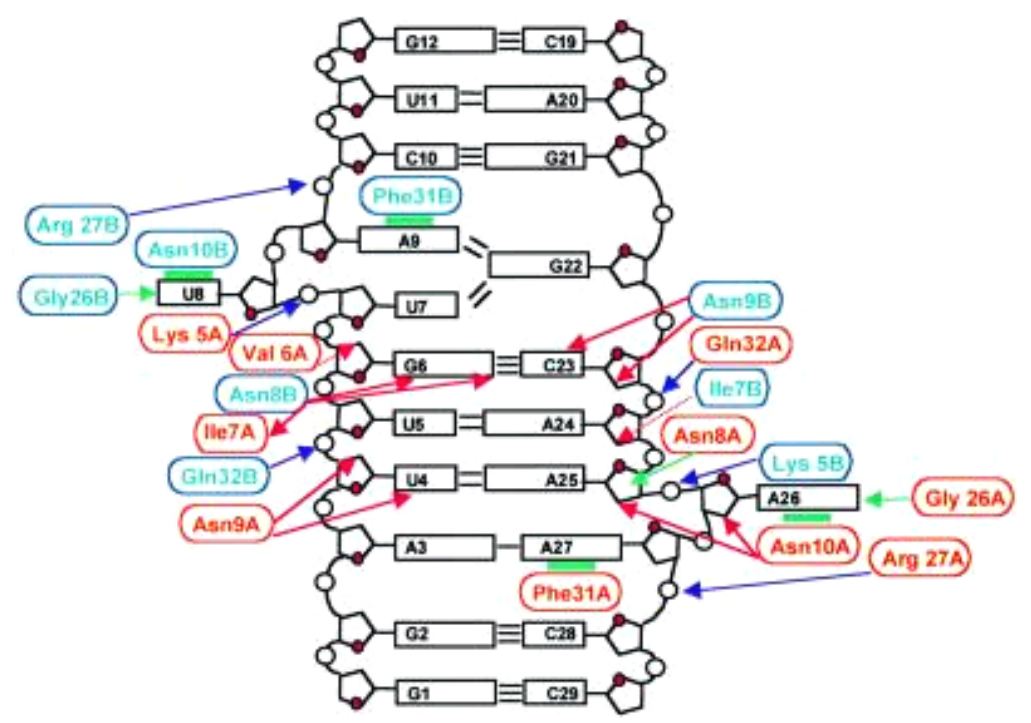

Figure 6.4. Schema of the direct molecular interactions of the LicT-bgIP RAT complex. Solid red lines indicate hydrogen bonds involving an amino acid side chain atom, dashed red lines indicate hydrogen bonds involving a protein backbone atom, green lines indicate van der Waals interactions and blue lines indicate possible interactions with the RNA phosphodiester backbone (Yang et al., 2002).

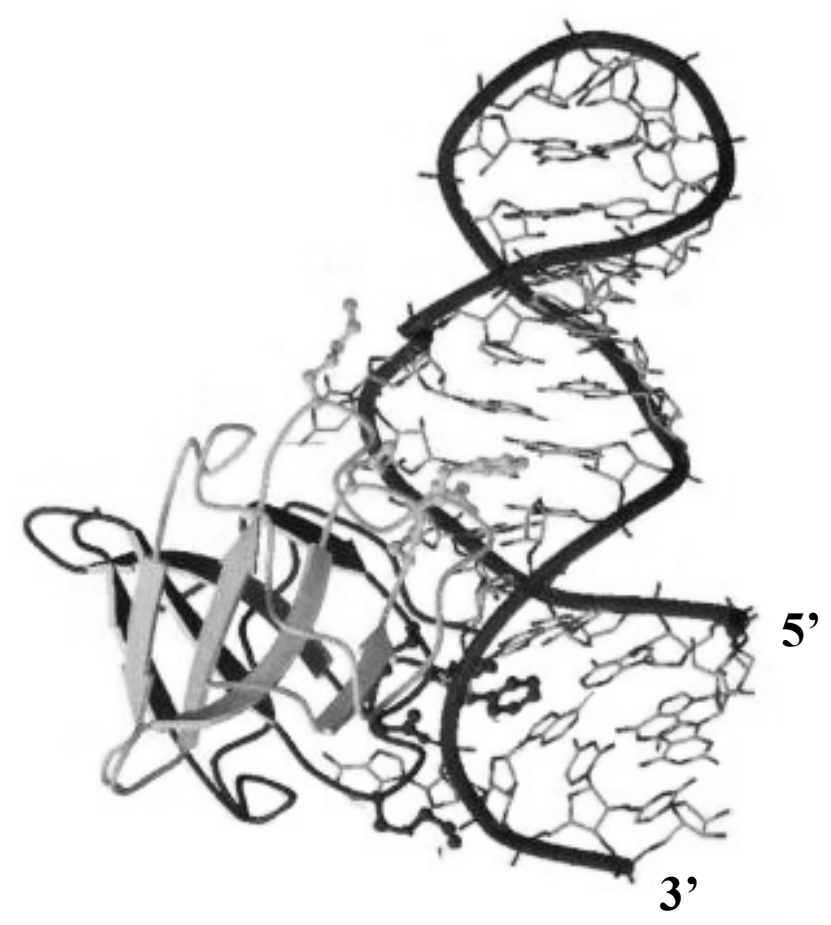

Figure 6.5. MOLSCRIPT (Kraulis, 1991) representation of the LicT dimer interacting with its RAT hairpin target. Modified from Yang et al., 2002. 
This is similar to the viral Rev-Peptide from HIV-1, which induces the splicing of a viral mRNA when binding to the RRE-sequence (Rev responsive element) (Daly et al., 1989; Zapp and Green, 1989). Here, the protein interacts also with the sugar phosphate backbone and several nucleotides located in the stem region of the RRE secondary structure. This interaction induces stem structure formation and is also essential for RNA-protein recognition (Bartel et al., 1991; Battiste et al., 1996; Battiste et al., 1994). Whether the interaction of LicT with the $\operatorname{bglP}$ middle stem-region is essential for RNA structure formation or not cannot be determined with the methods used in this study. However, with the exception of ptsG, the middle stem structure and sequence is identical for all the related RAT structures and is therefore not considered to be a specificity determinant. Thus, the lower loop region of the RATs seems to be the central element for the specific RNA-protein interaction.

Interestingly, a single nucleotide exchange in $s a c B$ RAT or the introduction of an additional nucleotide into pts $G$ RAT could change specificity towards another antiterminator protein. Something similar has been previously observed for metabolite dependent riboswitches. Guanine and adenine binding riboswitches are also very similar in structure and sequence and bind to their respective target with high affinity (Mandal et al., 2003; Mandal and Breaker, 2004) (see also chapter 1.2.3.3). The exchange of a single cytosine to uridine in its RNA sequence converts the guanine specific riboswitch to an adenine binding riboswitch (Mandal and Breaker, 2004). Similar to ptsG RAT, an unusual triple base pairing is responsible for specificity and target interaction (Noeske et al., 2005).

\section{The exceptional position of GlcT and ptsG-RAT}

GlcT and pts $G$ RAT hold an outstanding position since neither binds GlcT to any of the other related RAT sequences nor do any of the other antiterminator proteins bind to the pts $G$ RAT sequence. To understand the high specificity of the GlcT-pts $G$ RAT couple, a detailed comparison of all antiterminator systems is required. When having a closer look to the predicted secondary structures of the related RATs, it becomes apparent that $\operatorname{sacB}$, $\operatorname{sacPA}$, bglP, and licS RAT are all very similar in structure and sequence. On the other hand, pts $G$ RAT is more distant from the other RATs and exhibits some exclusive features.

First, the top loop features an additional nucleotide and a sequence (UUCGA) that is very different from the other four RAT top loop sequences (UAAA or GAAA, see Fig 6.3). Secondly, the middle stem region consists of only two base pairings instead of the three found in the other RATs. As the unpaired nucleotide at position U25 is part of the lower loop region, the $p t s G$ RAT lower loop comprises four nucleotides instead of three. Thirdly, the lower stem 
of pts $G$ RAT is unique and differs completely from the lower stem region of the other RATs (see Figs. 6.2, 6.3). Finally, a larger amount of nucleotides of the pts $G$ RAT sequence is overlapping with the terminator sequence (see Fig. 6.3).

The NMR structure of the LicT-bglP RAT complex clearly shows that the upper and lower loop regions are actually no open loop structures as shown in Figure 6.2 for ptsG RAT. Most nucleotides located in the loop regions are in unusual base pairings with opposing nucleotides. In the lower loop A3 interacts with A27 and in the upper loop region U7, A9, and G22 are forming a triple base pairing. Therefore, only the two nucleotides U8 and A26 remain unpaired. Thus, most of the nucleotides in the loop regions are participating in stem structure formation (see Fig. 6.4) (Yang et al., 2002). Since all related RAT structures, including ptsG RAT, feature the same nucleotide configuration in the top loop region, it is safe to propose the same triple base pairing for all RATs. The $p t s G$ RAT features this nucleotide composition also for the lower loop region (see Figs. 6.2 and 6.3). It is therefore predicted that the same triple base pairing formation is also present in the lower loop region forming a highly symmetrical RAT structure. When introducing this structural element into $s a c B$ RAT, binding of GlcT to the mutant RAT is observed. This confirms that this structural feature is indeed the specificity determinant for GlcT-ptsG RAT interaction.

When analyzing the evolution of the antiterminator protein regulatory domains it was found that GlcT of B. subtilis and Staphylococcus carnosus (Christiansen and Hengstenberg, 1996) is more distant from the antiterminator proteins of the sucrose and $\beta$-glucoside classes. The latter exhibit a closer relationship with each other and form a distinct subgroup (Greenberg et al., 2002). Thus, both the ptsG RAT and the GlcT antiterminator protein are the most distant components of the related antiterminator systems.

Considering all this, it becomes more clear why the GlcT-ptsG RAT interaction is the most specific of the related antitermination systems. The sugar transported by this system holds also an exclusive position. Glucose is the preferred carbon source of B. subtilis and when available, carbon catabolite repression suppresses genes needed for the utilization of alternative sugars. Eliminating any cross-talk is economical for the bacterium and helps to repress the expression of genes that are not required. Escherichia coli follows the same strategy. However, the control of PTS dependent glucose uptake differs fundamentally from that of B. subtilis. In E. coli, external glucose is also transported and phosphorylated via the PTS system (Meadow et al., 1990; Postma et al., 1993). The expression of the glucose permease PtsG is negatively controlled by the global regulator Mlc on the level of transcription initiation (Kimata et al., 1998; Tanaka et al., 1999). When glucose is transported 
into the cell, the membrane spanning domain of the glucose permease (enzyme IIBC ${ }^{\mathrm{Glc}}$ ) is mainly in a dephosphorylated state. Under these conditions, IIBC ${ }^{\mathrm{Glc}}$ binds Mlc resulting in sequestration of the transcription factor to the membrane (Lee et al., 2000; Tanaka et al., 2000). The membrane localization is the actual reason for Mlc inactivation (Tanaka et al., 2004). Additionally, PtsG synthesis is also regulated on a post-transcriptional level by modulating mRNA stability. The small non-coding RNA SgrS is expressed and, with the help of Hfq (see chapter 1.2.2), interacts with the 5'-end of the pts $G$ mRNA resulting in SgrS-pts $G$ base pairing (Vanderpool and Gottesman, 2004). The SgrS-ptsG complex is then subjected to RNase E-dependent degradation (Kawamoto et al., 2005; Kimata et al., 2001; Morita et al., 2004).

Although the implementation differs significantly, the PTS and regulatory RNA elements play a crucial role in both bacteria. Considering the special role of glucose within the PTS system, it can be assumed that the PTS evolved primarily as a glucose regulon. This was already proposed previously for E. coli (Plumbridge, 2002) and would give a plausible explanation for the outstanding position of glucose within the PTS in both bacteria. In addition, the PTS dependent antitermination systems of B. subtilis might also have evolved originally for the regulation of glucose uptake. However, the presence of PRD-II in GlcT, which is not of big regulatory relevance, could be an evidence for another origin of this system (Greenberg et al., 2002). Maybe an existing antitermination system regulating a sugarpermease that is under catabolite repression in the presence of glucose (like $s a c P A$ or bglPH) was the origin of the GlcT-ptsG system. In the original system, PRD-II might be needed for switching the system off when both the respective sugar and glucose are present simultaneously (see also chapter 1.3.3). When the system adapted to glucose uptake regulation, the PRD-II lost its relevance.

\section{More than just nucleotide-amino acid interaction mediates specificity}

Other factors than specific nucleotide-amino acid interaction seem to have influence on the specificity of the four antiterminator systems. Although not bound by the antiterminator proteins, the top loops seem to have indirect influence on gene expression. The nucleotide sequences and especially the nucleotides found at position 13 and 18 (or 12 and 18 in the case of $p t s G$ ), which are proposed to be in direct base pairing (see Fig. 6.3), are found to have influence on the expression level of the respective genes (data not shown). The GAAA tetraloop motive found in bglP RAT is very common in RNA secondary structures and is known to stabilize adjacent stem structures (Conn and Draper, 1998; Moore, 1999; Murphy 
and Cech, 1994). The exchange of a single nucleotide within the tetraloop can result in an overall destabilization of the RNA secondary structure. Thus, the configuration of the top loop has an indirect influence on the stability of the stem region and therefore on the expression level of the coding gene. The same was found for some nucleotides that are bound by the antiterminator proteins. It is proposed that $\mathrm{U} 7$ and G26 in ptsG RAT are in direct contact with amino acids of GlcT. Although the individual base is not relevant for interaction specificity, the type of nucleotide had an influence on the level of expression. An U7 U26 configuration resulted in a twofold higher expression rate, whereas a G7 G26 configuration resulted in a much lower expression rate in comparison to the wildtype RAT.

It was also found that nucleotides in the upper and lower loop region have a dual role by stabilizing the overall RAT structure and interacting with the respective protein. All RAT structures feature an adenine at position 27 (see Fig. 6.3) and this adenine is known to be in direct contact with the protein in the LicT-bglP RAT complex (see Fig. 6.4). Additionally, this nucleotide is in base pairing with an opposing $\mathrm{G}, \mathrm{U}$, or $\mathrm{A}$ and thus participating in stem structure formation. The Rev peptide of HIV-I is also recognizing an unusual GG base pairing in its target RNA. Furthermore, it was shown that binding of Rev induces the GG base pairing formation (Battiste et al., 1994). It was also reported that non Watson-Crick base pairings are necessary to maintain stem formation in double stranded RNA structures and induce RNA bending (Baeyens et al., 1996). The NMR structure of the LicT-bglP RAT complex clearly shows that the RNA structure is twisted (see Fig. 6.5) and the type of pairing at position 3 and 27 in the respective RAT might affect the torsion. Finally, the thermodynamic stability of the terminator structure has also influence on the overall expression rate of the coding genes. Considering all this, it is hard to separate the effects coming from a single nucleotide exchange. It could affect the stability of the RAT secondary structure, the strength of RATprotein interaction, the torsion of the RAT structure, or, when compensatory mutations were introduced, the stability of the terminator structure.

In their active state, all four antiterminator proteins form dimers from identical subunits. As the monomers of the cognate proteins share a high homology among each other, it is reasonable to wonder whether the belonging subunits specifically interact with each other or whether heterodimers formed from different subunits might exist. The data obtained from site directed mutagenesis of $s a c B$ RAT revealed that one of the RAT mutants could only cause antitermination when both SacT and SacY were present and active. The deletion of either SacT or SacY resulted in transcription termination of the relevant RAT mutant (data not shown). Although this proposed heterodimer does not seem to interact with any of the wild 
type RAT structures, it might be possible that this interaction helps to fine-tune the activity of SacT and SacY under certain conditions by forming inactive heterodimers.

Furthermore, there still seem to be unknown factors involved in specificity determination. SacT was found to bind to all $\operatorname{sac} B$ derived RAT structures. However, it is not reported that the presence of sucrose results in SacT dependent expression of bglPH or licS. On the other hand, a mutant RAT structure that was derived from $p t s G$ RAT sequence and was changed towards $\operatorname{sacPA}$ RAT sequence exhibited strong LicT binding and only minor interaction with SacT was observed. This could indicate that sequences other than RAT and terminator might be relevant for the specific interaction of some RAT-protein couples. It is also noteworthy that the RNA-protein recognition is a highly dynamic process occurring while the RNA is just originating. The sequence upstream of the respective RAT might be involved in RAT structure formation or in guiding the antiterminator protein to the nascent RAT sequence. However, the dynamic RNA synthesis and folding process cannot be monitored in detail. 


\subsection{Outlook}

The results in this work clearly show the influence of the organic acids glutamate and succinate on the central metabolism of B. subtilis when given in addition to glucose. The data obtained from the two methods used, cDNA microarray analysis and metabolic flux analysis, are in good agreement for most metabolic pathways. However, the data obtained with the microarray analysis for $p c k A$ and some overflow metabolic pathways are not congruent with the data gained from the metabolic flux analysis. This could indicate that the relevant enzymes are regulated rather on the level of protein activity than on the level of transcription or translation. Classical molecular biological methods and 2D gelelectrophoresis could give further evidence for the mode of regulation of these enzymes.

Furthermore, two modern and powerful screening methods were established and optimized for the analysis of the central metabolic pathways of B. subtilis. With these methods at hand, further analysis of the influence of nutrients on the central metabolism of $B$ subtilis is a comparatively easy task.

The mutational analysis of the ptsG and $s a c B$ RAT sequences revealed the specificity determinants of all related RAT-protein couples. However, it is still unclear if other sequences than RAT and terminator might be involved in the dynamic process of RAT folding and protein recognition. The potential of site directed mutagenesis also comes to its limits as the consequence of a single nucleotide exchange cannot be studied isolated from side effects (see chapter 6.2). Therefore, it is desirable to obtain crystal or NMR structures of the RAT mutants in complex with the recognized antiterminator protein. Moreover, a mutational analysis of the antiterminator proteins could give further insights into RNA-protein recognition and could elucidate how RNA and protein adapted to target recognition.

B. subtilis is able to grow in the range of $12{ }^{\circ} \mathrm{C}$ to $52^{\circ} \mathrm{C}$. The temperature sensitive RNA switches (see chapter 1.2.3.1) clearly indicate that even a small shift in temperature can alter RNA secondary structure. It might be interesting to study whether the PTS controlled antitermination systems are functional within the entire temperature range and how the systems are affected by heavy temperature shifts. The RATs and antiterminator proteins of thermophilic bacilli could be studied to address these questions. 


\section{References}

Aigner, A. 2006. Gene silencing through RNA interference (RNAi) in vivo: strategies based on the direct application of siRNAs. J Biotechnol 124:12-25.

Akashi, H. and T. Gojobori. 2002. Metabolic efficiency and amino acid composition in the proteomes of Escherichia coli and Bacillus subtilis. Proc Natl Acad Sci USA 99:3695-3700.

Albright, L. M., E. Huala and F. M. Ausubel. 1989. Prokaryotic signal transduction mediated by sensor and regulator protein pairs. Annu Rev Genet 23:311-336.

Amarzguioui, M., J. J. Rossi and D. Kim. 2005. Approaches for chemically synthesized siRNA and vector-mediated RNAi. FEBS Lett 579:5974-5981.

Ambros, V. 2004. The functions of animal microRNAs. Nature 431:350-355.

Amster-Choder, O., F. Houman and A. Wright. 1989. Protein phosphorylation regulates transcription of the beta-glucoside utilization operon in E. coli. Cell 58:847-855.

Amster-Choder, O. and A. Wright. 1992. Modulation of the dimerization of a transcriptional antiterminator protein by phosphorylation. Science 257:1395-1398.

Amster-Choder, O. and A. Wright. 1993. Transcriptional regulation of the $b g l$ operon of Escherichia coli involves phosphotransferase system-mediated phosphorylation of a transcriptional antiterminator. $J$ Cell Biochem 51:83-90.

Antson, A. A., E. J. Dodson, G. Dodson, R. B. Greaves, X. Chen and P. Gollnick. 1999. Structure of the trp RNA-binding attenuation protein, TRAP, bound to RNA. Nature 401:235-242.

Antson, A. A., J. Otridge, A. M. Brzozowski, E. J. Dodson, G. G. Dodson, K. S. Wilson, T. M. Smith, M. Yang, T. Kurecki and P. Gollnick. 1995. The structure of trp RNAbinding attenuation protein. Nature 374:693-700.

Aravin, A. and T. Tuschl. 2005. Identification and characterization of small RNAs involved in RNA silencing. FEBS Lett 579:5830-5840.

Arnaud, M., M. Débarbouillé, G. Rapoport, M. H. Saier and J. Reizer. 1996. In vitro reconstitution of transcriptional antitermination by the SacT and SacY proteins of Bacillus subtilis. J Biol Chem 271:18966-18972.

Arnaud, M., P. Vary, M. Zagorec, A. Klier, M. Débarbouillé, P. Postma and G. Rapoport. 1992. Regulation of the sacPA operon of Bacillus subtilis: identification of phosphotransferase system components involved in SacT activity. J Bacteriol 174:3161-3170. 
Asai, K., S. H. Baik, Y. Kasahara, S. Moriya and N. Ogasawara. 2000. Regulation of the transport system for C4-dicarboxylic acids in Bacillus subtilis. Microbiology 146:263-271.

Aymerich, S. and M. Steinmetz. 1992. Specificity determinants and structural features in the RNA target of the bacterial antiterminator proteins of the BglG/SacY family. Proc Natl Acad Sci USA 89:10410-10414.

Babitzke, P. 2004. Regulation of transcription attenuation and translation initiation by allosteric control of an RNA-binding protein: the Bacillus subtilis TRAP protein. Curr Opin Microbiol 7:132-139.

Babitzke, P. and P. Gollnick. 2001. Posttranscription initiation control of tryptophan metabolism in Bacillus subtilis by the trp RNA-binding attenuation protein (TRAP), antiTRAP, and RNA structure. J Bacteriol 183:5795-5802.

Babitzke, P., J. Yealy and D. Campanelli. 1996. Interaction of the trp RNA-binding attenuation protein (TRAP) of Bacillus subtilis with RNA: effects of the number of GAG repeats, the nucleotides separating adjacent repeats, and RNA secondary structure. $J$ Bacteriol 178:5159-5163.

Bachem, S., N. Faires and J. Stülke. 1997. Characterization of the presumptive phosphorylation sites of the Bacillus subtilis glucose permease by site-directed mutagenesis: implication in glucose transport and catabolite repression. FEMS Microbiol Lett 156:233-238.

Bachem, S. and J. Stülke. 1998. Regulation of the Bacillus subtilis GlcT antiterminator protein by components of the phosphotransferase system. J Bacteriol 180:5319-5326.

Bae, W., B. Xia, M. Inouye and K. Severinov. 2000. Escherichia coli CspA-family RNA chaperones are transcription antiterminators. Proc Natl Acad Sci USA 97:7784-7789.

Baeyens, K. J., H. L. De Bondt, A. Pardi and S. R. Holbrook. 1996. A curved RNA helix incorporating an internal loop with G'A and A A non-Watson-Crick base pairing. Proc Natl Acad Sci U S A 93:12851-12855.

Barash, D. 2003. Deleterious mutation prediction in the secondary structure of RNAs. Nucleic Acids Res 31:6578-6584.

Barrick, J. E., K. A. Corbino, W. C. Winkler, A. Nahvi, M. Mandal, J. Collins, M. Lee, A. Roth, N. Sudarsan, I. Jona, J. K. Wickiser and R. R. Breaker. 2004. New RNA motifs suggest an expanded scope for riboswitches in bacterial genetic control. Proc Natl Acad Sci USA 101:6421-6426.

Bartel, D. P., M. L. Zapp, M. R. Green and J. W. Szostak. 1991. HIV-1 Rev regulation involves recognition of non-Watson-Crick base pairs in viral RNA. Cell 67:529-536.

Battiste, J. L., H. Mao, N. S. Rao, R. Tan, D. R. Muhandiram, L. E. Kay, A. D. Frankel and J. R. Williamson. 1996. $\alpha$ Helix-RNA major groove recognition in an HIV-1 Rev peptide-RRE RNA complex. Science 273:1547-1551. 
Battiste, J. L., R. Y. Tan, A. D. Frankel and J. R. Williamson. 1994. Binding of an HIV Rev peptide to Rev responsive element RNA induces formation of purine-purine base pairs. Biochemistry 33:2741-2747.

Baumann, C., S. Xirasagar and P. Gollnick. 1997. The trp RNA-binding attenuation protein (TRAP) from Bacillus subtilis binds to unstacked trp leader RNA. J Biol Chem 272:19863-19869.

Begley, T. P., D. M. Downs, S. E. Ealick, F. W. McLafferty, A. P. Van Loon, S. Taylor, N. Campobasso, H. J. Chiu, C. Kinsland, J. J. Reddick and J. Xi. 1999. Thiamin biosynthesis in prokaryotes. Arch Microbiol 171:293-300.

Belitsky, B. R. and A. L. Sonenshein. 1998. Role and regulation of Bacillus subtilis glutamate dehydrogenase genes. J Bacteriol 180:6298-6305.

Belitsky, B. R., L. V. Wray, Jr., S. H. Fisher, D. E. Bohannon and A. L. Sonenshein. 2000. Role of TnrA in nitrogen source-dependent repression of Bacillus subtilis glutamate synthase gene expression. J Bacteriol 182:5939-5947.

Bernhardt, J., J. Weibezahn, C. Scharf and M. Hecker. 2003. Bacillus subtilis during feast and famine: visualization of the overall regulation of protein synthesis during glucose starvation by proteome analysis. Genome Res 13:224-237.

Bi, W. and P. J. Stambrook. 1997. CCR: a rapid and simple approach for mutation detection. Nucleic Acids Res 25:2949-2951.

Bi, W. and P. J. Stambrook. 1998. Site-directed mutagenesis by combined chain reaction. Anal Biochem 256:137-140.

Black, D. L. 2000. Protein diversity from alternative splicing: a challenge for bioinformatics and post-genome biology. Cell 103:367-370.

Blank, L. M. and U. Sauer. 2004. TCA cycle activity in Saccharomyces cerevisiae is a function of the environmentally determined specific growth and glucose uptake rates. Microbiology 150:1085-1093.

Blencke, H.-M., G. Homuth, H. Ludwig, U. Mäder, M. Hecker and J. Stülke. 2003. Transcriptional profiling of gene expression in response to glucose in Bacillus subtilis: regulation of the central metabolic pathways. Metab Eng 5:133-149.

Blencke, H.-M., I. Reif, F. M. Commichau, C. Detsch, I. Wacker, H. Ludwig and J. Stülke. 2006. Regulation of citB expression in Bacillus subtilis: integration of multiple metabolic signals in the citrate pool and by the general nitrogen regulatory system. Arch Microbiol 185: 136-146.

Brantl, S. 2002. Antisense RNAs in plasmids: control of replication and maintenance. Plasmid 48:165-173. 
Bugala, K., M. Zywicki, E. Wyszko, M. Z. Barciszewska and J. Barciszewski. 2005. Riboswitches. Postepy Biochem 51:111-119.

Burbulys, D., K. A. Trach and J. A. Hoch. 1991. Initiation of sporulation in B. subtilis is controlled by a multicomponent phosphorelay. Cell 64:545-552.

Canonaco, F., T. A. Hess, S. Heri, T. Wang, T. Szyperski and U. Sauer. 2001. Metabolic flux response to phosphoglucose isomerase knock-out in Escherichia coli and impact of overexpression of the soluble transhydrogenase UdhA. FEMS Microbiol Lett 204:247-252.

Carthew, R. W. 2006. Gene regulation by microRNAs. Curr Opin Genet Dev 16:203-208.

Chai, W. and V. Stewart. 1998. NasR, a novel RNA-binding protein, mediates nitrateresponsive transcription antitermination of the Klebsiella oxytoca M5al nasF operon leader in vitro. J Mol Biol 283:339-351.

Chai, W. and V. Stewart. 1999. RNA sequence requirements for NasR-mediated, nitrateresponsive transcription antitermination of the Klebsiella oxytoca M5al nasF operon leader. $J$ Mol Biol 292:203-216.

Chambliss, G. H. 1993. Carbon source-mediated catabolite repression. In: Bacillus subtilis and other gram-positive bacteria, biochemistry, physiology, and molecular genetics. A. L. Sonenshein, J. A. Hoch and R. Losick. (eds.), ASM press, Washington DC, pp. 213-219.

Chander, P., K. M. Halbig, J. K. Miller, C. J. Fields, H. K. Bonner, G. K. Grabner, R. L. Switzer and J. L. Smith. 2005. Structure of the nucleotide complex of PyrR, the pyr attenuation protein from Bacillus caldolyticus, suggests dual regulation by pyrimidine and purine nucleotides. J Bacteriol 187:1773-1782.

Chaudhry, R., N. Nisar, B. Hora, S. R. Chirasani and P. Malhotra. 2005. Expression and immunological characterization of the carboxy-terminal region of the P1 adhesin protein of Mycoplasma pneumoniae. J Clin Microbiol 43:321-325.

Christensen, B., A. K. Gombert and J. Nielsen. 2002. Analysis of flux estimates based on ${ }^{13}$ C-labelling experiments. Eur J Biochem 269:2795-2800.

Christiansen, I. and W. Hengstenberg. 1996. Cloning and sequencing of two genes from Staphylococcus carnosus coding for glucose-specific PTS and their expression in Escherichia coli K-12. Mol Gen Genet 250:375-379.

Christiansen, I. and W. Hengstenberg. 1999. Staphylococcal phosphoenolpyruvatedependent phosphotransferase system - two highly similar glucose permeases in Staphylococcus carnosus with different glucoside specificity: protein engineering in vivo? Microbiology 145:2881-2889.

Commichau, F. M., K. Forchhammer and J. Stülke. 2006. Regulatory links between carbon and nitrogen metabolism. Curr Opin Microbiol 9:167-172. 
Condon, C., M. Grunberg-Manago and H. Putzer. 1996. Aminoacyl-tRNA synthetase gene regulation in Bacillus subtilis. Biochimie 78:381-389.

Conn, G. L. and D. E. Draper. 1998. RNA structure. Curr Opin Struct Biol 8:278-285.

Covert, M. W., C. H. Schilling, I. Famili, J. S. Edwards, Goryanin, II, E. Selkov and B. O. Palsson. 2001. Metabolic modeling of microbial strains in silico. Trends Biochem Sci 26:179-186.

Crutz, A. M., M. Steinmetz, S. Aymerich, R. Richter and D. Le Coq. 1990. Induction of levansucrase in Bacillus subtilis: an antitermination mechanism negatively controlled by the phosphotransferase system. J Bacteriol 172:1043-1050.

Cruz Ramos, H., T. Hoffmann, M. Marino, H. Nedjari, E. Presecan-Siedel, O. Dreesen, P. Glaser and D. Jahn. 2000. Fermentative metabolism of Bacillus subtilis: physiology and regulation of gene expression. $J$ Bacteriol 182:3072-3080.

Daly, T. J., K. S. Cook, G. S. Gray, T. E. Maione and J. R. Rusche. 1989. Specific binding of HIV-1 recombinant Rev protein to the Rev-responsive element in vitro. Nature 342:816819.

Darbon, E., P. Servant, S. Poncet and J. Deutscher. 2002. Antitermination by GlpP, catabolite repression via CcpA and inducer exclusion triggered by $\mathrm{P} \sim \mathrm{GlpK}$ dephosphorylation control Bacillus subtilis glpFK expression. Mol Microbiol 43:1039-1052.

Dauner, M., J. E. Bailey and U. Sauer. 2001. Metabolic flux analysis with a comprehensive isotopomer model in Bacillus subtilis. Biotechnol Bioeng 76:144-156.

Dauner, M. and U. Sauer. 2001. Stoichiometric growth model for riboflavin-producing Bacillus subtilis. Biotechnol Bioeng 76:132-143.

Dauner, M., M. Sonderegger, M. Hochuli, T. Szyperski, K. Wüthrich, H. P. Hohmann, U. Sauer and J. E. Bailey. 2002. Intracellular carbon fluxes in riboflavin-producing Bacillus subtilis during growth on two-carbon substrate mixtures. Appl Environ Microbiol 68:17601771.

Débarbouillé, M., M. Arnaud, A. Fouet, A. Klier and G. Rapoport. 1990. The sacT gene regulating the $s a c P A$ operon in Bacillus subtilis shares strong homology with transcriptional antiterminators. J Bacteriol 172:3966-3973.

Declerck, N., F. Vincent, F. Hoh, S. Aymerich and H. van Tilbeurgh. 1999. RNA recognition by transcriptional antiterminators of the $\mathrm{BglG} / \mathrm{SacY}$ family: functional and structural comparison of the CAT domain from SacY and LicT. J Mol Biol 294:389-402.

Desai, S. K. and J. P. Gallivan. 2004. Genetic screens and selections for small molecules based on a synthetic riboswitch that activates protein translation. J Am Chem Soc 126:1324713254. 
Detsch, C. and J. Stülke. 2003. Ammonium utilization in Bacillus subtilis: transport and regulatory functions of NrgA and NrgB. Microbiology 149:3289-3297.

Deutscher, J., B. Pevec, K. Beyreuther, H. H. Kiltz and W. Hengstenberg. 1986. Streptococcal phosphoenolpyruvate-sugar phosphotransferase system: amino acid sequence and site of ATP-dependent phosphorylation of HPr. Biochemistry 25:6543-6551.

Deutscher, J. and M. H. Saier, Jr. 1983. ATP-dependent protein kinase-catalyzed phosphorylation of a seryl residue in HPr, a phosphate carrier protein of the phosphotransferase system in Streptococcus pyogenes. Proc Natl Acad Sci USA 80:67906794.

Diesterhaft, M. D. and E. Freese. 1973. Role of pyruvate carboxylase, phosphoenolpyruvate carboxykinase, and malic enzyme during growth and sporulation of Bacillus subtilis. J Biol Chem 248:6062-6070.

Doan, T. and S. Aymerich. 2003. Regulation of the central glycolytic genes in Bacillus subtilis: binding of the repressor CggR to its single DNA target sequence is modulated by fructose-1,6-bisphosphate. Mol Microbiol 47:1709-1721.

Doan, T., P. Servant, S. Tojo, H. Yamaguchi, G. Lerondel, K. Yoshida, Y. Fujita and S. Aymerich. 2003. The Bacillus subtilis ywkA gene encodes a malic enzyme and its transcription is activated by the YufL/YufM two-component system in response to malate. Microbiology 149:2331-2343.

Domingo, E. and J. J. Holland. 1997. RNA virus mutations and fitness for survival. Annu Rev Microbiol 51:151-178.

Eigen, M., W. Gardiner, P. Schuster and R. Winkler-Oswatitsch. 1981. The origin of genetic information. Sci Am 244:88-92.

El-Mansi, M., A. J. Cozzone, J. Shiloach and B. J. Eikmanns. 2006. Control of carbon flux through enzymes of central and intermediary metabolism during growth of Escherichia coli on acetate. Curr Opin Microbiol 9:173-179.

Ellington, A. D. and J. W. Szostak. 1990. In vitro selection of RNA molecules that bind specific ligands. Nature 346:818-822.

Evans, P. R., G. W. Farrants and P. J. Hudson. 1981. Phosphofructokinase: structure and control. Philos Trans R Soc Lond B Biol Sci 293:53-62.

Fabret, C., V. A. Feher and J. A. Hoch. 1999. Two-component signal transduction in Bacillus subtilis: how one organism sees its world. J Bacteriol 181:1975-1983.

Faires, N., S. Tobisch, S. Bachem, I. Martin-Verstraete, M. Hecker and J. Stülke. 1999. The catabolite control protein CcpA controls ammonium assimilation in Bacillus subtilis. $J$ Mol Microbiol Biotechnol 1:141-148. 
Fang, L., W. Jiang, W. Bae and M. Inouye. 1997. Promoter-independent cold-shock induction of $\operatorname{csp} A$ and its derepression at $37^{\circ} \mathrm{C}$ by mRNA stabilization. Mol Microbiol 23:355-364.

Fauzi, H., K. D. Jack and J. V. Hines. 2005. In vitro selection to identify determinants in tRNA for Bacillus subtilis tyrS $\mathrm{T}$ box antiterminator mRNA binding. Nucleic Acids Res 33:2595-2602.

Fillinger, S., S. Boschi-Muller, S. Azza, E. Dervyn, G. Branlant and S. Aymerich. 2000. Two glyceraldehyde-3-phosphate dehydrogenases with opposite physiological roles in a nonphotosynthetic bacterium. J Biol Chem 275:14031-14037.

Fisher, S. H. 1999. Regulation of nitrogen metabolism in Bacillus subtilis: vive la différence! Mol Microbiol 32:223-232.

Fisher, S. H. and B. Magasanik. 1984. 2-Ketoglutarate and the regulation of aconitase and histidase formation in Bacillus subtilis. J Bacteriol 158:379-382.

Fujita, Y., K. Yoshida, Y. Miwa, N. Yanai, E. Nagakawa and Y. Kasahara. 1998. Identification and expression of the Bacillus subtilis fructose-1,6-bisphosphatase gene $(f b p) . J$ Bacteriol 180:4309-4313.

Fux, L., A. Nussbaum-Shochat and O. Amster-Choder. 2003. Interactions between the PTS regulation domains of the BglG transcriptional antiterminator from Escherichia coli. $J$ Biol Chem 278:46203-46209.

Galinier, A., J. Deutscher and I. Martin-Verstraete. 1999. Phosphorylation of either Crh or HPr mediates binding of CcpA to the Bacillus subtilis xyn cre and catabolite repression of the xyn operon. J Mol Biol 286:307-314.

Galinier, A., J. Haiech, M. C. Kilhoffer, M. Jaquinod, J. Stülke, J. Deutscher and I. Martin-Verstraete. 1997. The Bacillus subtilis crh gene encodes a HPr-like protein involved in carbon catabolite repression. Proc Natl Acad Sci USA 94:8439-8444.

Galinier, A., M. Kravanja, R. Engelmann, W. Hengstenberg, M. C. Kilhoffer, J. Deutscher and J. Haiech. 1998. New protein kinase and protein phosphatase families mediate signal transduction in bacterial catabolite repression. Proc Natl Acad Sci USA 95:1823-1828.

Galvao, T. C. and V. de Lorenzo. 2006. Transcriptional regulators à la carte: engineering new effector specificities in bacterial regulatory proteins. Curr Opin Biotechnol 17:34-42.

Gardan, R., G. Rapoport and M. Débarbouillé. 1995. Expression of the rocDEF operon involved in arginine catabolism in Bacillus subtilis. J Mol Biol 249:843-856.

Garmendia, J., D. Devos, A. Valencia and V. de Lorenzo. 2001. À la carte transcriptional regulators: unlocking responses of the prokaryotic enhancer-binding protein XylR to nonnatural effectors. Mol Microbiol 42:47-59. 
Gautheret, D. and R. R. Gutell. 1997. Inferring the conformation of RNA base pairs and triples from patterns of sequence variation. Nucleic Acids Res 25:1559-1564.

Gelfand, M. S., A. A. Mironov, J. Jomantas, Y. I. Kozlov and D. A. Perumov. 1999. A conserved RNA structure element involved in the regulation of bacterial riboflavin synthesis genes. Trends Genet 15:439-442.

Gerdeman, M. S., T. M. Henkin and J. V. Hines. 2003. Solution structure of the Bacillus subtilis T-box antiterminator RNA: seven nucleotide bulge characterized by stacking and flexibility. J Mol Biol 326:189-201.

Gilbert, W. 1986. The RNA world. Nature (London) 319:618.

Gollnick, P. 1994. Regulation of the Bacillus subtilis trp operon by an RNA-binding protein. Mol Microbiol 11:991-997.

Gollnick, P., C. Baumann, M. Yang, J. Otridge and A. Antson. 1995. Interaction of the 11-subunit trp RNA-binding attenuation protein (TRAP) with its RNA target. Nucleic Acids Symp Ser 43-45.

Gonzy-Tréboul, M. Zagorec, M. C. Rain-Guion and M. Steinmetz. 1989. Phosphoenolpyruvate:sugar phosphotransferase system of Bacillus subtilis: nucleotide sequence of $p t s X, p t s H$ and the 5'-end of ptsI and evidence for a ptsHI operon. Mol Microbiol 3:103-112.

Görke, B., L. Fraysse and A. Galinier. 2004. Drastic differences in Crh and HPr synthesis levels reflect their different impacts on catabolite repression in Bacillus subtilis. J Bacteriol 186:2992-2995.

Gottesman, S. 2004. The small RNA regulators of Escherichia coli: roles and mechanisms. Annu Rev Microbiol 58:303-328.

Gottesman, S. 2005. Micros for microbes: non-coding regulatory RNAs in bacteria. Trends Genet 21:399-404.

Goudreau, P. N. and A. M. Stock. 1998. Signal transduction in bacteria: molecular mechanisms of stimulus-response coupling. Curr Opin Microbiol 1:160-169.

Grabner, G. K. and R. L. Switzer. 2003. Kinetic studies of the uracil phosphoribosyltransferase reaction catalyzed by the Bacillus subtilis pyrimidine attenuation regulatory protein PyrR. J Biol Chem 278:6921-6927.

Grahn, E., N. J. Stonehouse, J. B. Murray, S. van den Worm, K. Valegård, K. Fridborg, P. G. Stockley and L. Liljas. 1999. Crystallographic studies of RNA hairpins in complexes with recombinant MS2 capsids: implications for binding requirements. RNA 5:131-138. 
Greenberg, D. B., J. Stülke and M. H. Saier, Jr. 2002. Domain analysis of transcriptional regulators bearing PTS regulatory domains. Res Microbiol 153:519-526.

Grundy, F. J., J. A. Collins, S. M. Rollins and T. M. Henkin. 2000. tRNA determinants for transcription antitermination of the Bacillus subtilis tyrS gene. RNA 6:1131-1141.

Grundy, F. J. and T. M. Henkin. 1993. tRNA as a positive regulator of transcription antitermination in B. subtilis. Cell 74:475-482.

Grundy, F. J. and T. M. Henkin. 1998. The S box regulon: a new global transcription termination control system for methionine and cysteine biosynthesis genes in gram-positive bacteria. Mol Microbiol 30:737-749.

Grundy, F. J. and T. M. Henkin. 2003. The T box and $\mathrm{S}$ box transcription termination control systems. Front Biosci 8:20-31.

Grundy, F. J. and T. M. Henkin. 2004. Regulation of gene expression by effectors that bind to RNA. Curr Opin Microbiol 7:126-131.

Grundy, F. J., S. E. Hodil, S. M. Rollins and T. M. Henkin. 1997. Specificity of tRNAmRNA interactions in Bacillus subtilis tyrS antitermination. J Bacteriol 179:2587-2594.

Grundy, F. J., S. M. Rollins and T. M. Henkin. 1994. Interaction between the acceptor end of tRNA and the T box stimulates antitermination in the Bacillus subtilis tyrS gene: a new role for the discriminator base. $J$ Bacteriol 176:4518-4526.

Grundy, F. J., D. A. Waters, S. H. Allen and T. M. Henkin. 1993. Regulation of the Bacillus subtilis acetate kinase gene by CcpA. J Bacteriol 175:7348-7355.

Grundy, F. J., W. C. Winkler and T. M. Henkin. 2002. tRNA-mediated transcription antitermination in vitro: codon-anticodon pairing independent of the ribosome. Proc Natl Acad Sci USA 99:11121-11126.

Guérout-Fleury, A. M., K. Shazand, N. Frandsen and P. Stragier. 1995. Antibioticresistance cassettes for Bacillus subtilis. Gene 167:335-336.

Guillier, M., S. Gottesman and G. Storz. 2006. Modulating the outer membrane with small RNAs. Genes Dev 20:2338-2348.

Halbedel, S., C. Hames and J. Stülke. 2004. In vivo activity of enzymatic and regulatory components of the phosphoenolpyruvate:sugar phosphotransferase system in Mycoplasma pneumoniae. J Bacteriol 186:7936-7943.

Hames, C., S. Halbedel, O. Schilling and J. Stülke. 2005. Multiple-mutation reaction: a method for simultaneous introduction of multiple mutations into the $g l p K$ gene of Mycoplasma pneumoniae. Appl Environ Microbiol 71:4097-4100. 
Hanson, K. G., K. Steinhauer, J. Reizer, W. Hillen and J. Stülke. 2002. HPr kinase/phosphatase of Bacillus subtilis: expression of the gene and effects of mutations on enzyme activity, growth and carbon catabolite repression. Microbiology 148:1805-1811.

Hanson, S., G. Bauer, B. Fink and B. Suess. 2005. Molecular analysis of a synthetic tetracycline-binding riboswitch. RNA 11:503-511.

Harrington, C. A., C. Rosenow and J. Retief. 2000. Monitoring gene expression using DNA microarrays. Curr Opin Microbiol 3:285-291.

Harwood, C. R. 1992. Bacillus subtilis and its relatives: molecular biological and industrial workhorses. Trends Biotechnol 10:247-256.

Hecker, M. 2003. A proteomic view of cell physiology of Bacillus subtilis-bringing the genome sequence to life. Adv Biochem Eng Biotechnol 83:57-92.

Hecker, M. and S. Engelmann. 2000. Proteomics, DNA arrays and the analysis of still unknown regulons and unknown proteins of Bacillus subtilis and pathogenic gram-positive bacteria. Int J Med Microbiol 290:123-134.

Hederstedt, L. 1993. The Krebs Citric Acid Cycle. In: Bacillus subtilis and other grampositive bacteria, biochemistry, physiology, and molecular genetics. A. L. Sonenshein, J. A. Hoch and R. Losick. (eds.), ASM press, Washington DC, pp. 181-197.

Heidrich, N., A. Chinali, U. Gerth and S. Brantl. 2006. The small untranslated RNA SR1 from the Bacillus subtilis genome is involved in the regulation of arginine catabolism. Mol Microbiol 62:520-536.

Helmann, J. D. 1999. Anti-sigma factors. Curr Opin Microbiol 2:135-141.

Hemilä, H., A. Palva, L. Paulin, L. Adler, S. Arvidson and I. Palva. 1991. The secretory S complex in Bacillus subtilis is identified as pyruvate dehydrogenase. Res Microbiol 142:779785 .

Henkin, T. M. 1996. The role of the CcpA transcriptional regulator in carbon metabolism in Bacillus subtilis. FEMS Microbiol Lett 135:9-15.

Henkin, T. M. 2000. Transcription termination control in bacteria. Curr Opin Microbiol 3:149-153.

Henkin, T. M., F. J. Grundy, W. L. Nicholson and G. H. Chambliss. 1991. Catabolite repression of alpha-amylase gene expression in Bacillus subtilis involves a trans-acting gene product homologous to the Escherichia coli lacI and galR repressors. Mol Microbiol 5:575584 . 
Henkin, T. M. and C. Yanofsky. 2002. Regulation by transcription attenuation in bacteria: how RNA provides instructions for transcription termination/antitermination decisions. Bioessays 24:700-707.

Hermann, T. and D. J. Patel. 2000. Adaptive recognition by nucleic acid aptamers. Science 287:820-825.

Hesselberth, J. R., M. P. Robertson, S. M. Knudsen and A. D. Ellington. 2003. Simultaneous detection of diverse analytes with an aptazyme ligase array. Anal Biochem 312:106-112.

Himmelreich, R., H. Hilbert, H. Plagens, E. Pirkl, B.-C. Li and R. Herrmann. 1996. Complete sequence analysis of the genome of the bacterium Mycoplasma pneumoniae. Nucleic Acids Res 24:4420-4449.

Hoch, J. A. and K. I. Varughese. 2001. Keeping signals straight in phosphorelay signal transduction. J Bacteriol 183:4941-4949.

Holtzclaw, W. D. and L. F. Chapman. 1975. Degradative acetolactate synthase of Bacillus subtilis: purification and properties. J Bacteriol 121:917-922.

Hovey, R., S. Lentes, A. Ehrenreich, K. Salmon, K. Saba, G. Gottschalk, R. P. Gunsalus and U. Deppenmeier. 2005. DNA microarray analysis of Methanosarcina mazei Gö1 reveals adaptation to different methanogenic substrates. Mol Genet Genomics 273:225-239.

Hueck, C. J., W. Hillen and M. H. Saier, Jr. 1994. Analysis of a cis-active sequence mediating catabolite repression in gram-positive bacteria. Res Microbiol 145:503-518.

Huffman, J. L. and R. G. Brennan. 2002. Prokaryotic transcription regulators: more than just the helix-turn-helix motif. Curr Opin Struct Biol 12:98-106.

Hutchison III, C. A., S. N. Peterson, S. R. Gill, R. T. Cline, O. White, C. M. Fraser, H. O. Smith and J. C. Venter. 1999. Global transposon mutagenesis and a minimal mycoplasma genome. Science 286:2165-2169.

Huynen, M. A., T. Dandekar and P. Bork. 1999. Variation and evolution of the citric-acid cycle: a genomic perspective. Trends Microbiol 7:281-291.

Inamine, J. M., K.-C. Ho, S. Loechel and P.-C. Hu. 1990. Evidence that UGA is read as a tryptophan codon rather than as a stop codon by Mycoplasma pneumoniae, Mycoplasma genitalium, and Mycoplasma gallisepticum. J Bacteriol 172:504-506.

Jacob, F. and J. Monod. 1961. Genetic regulatory mechanisms in the synthesis of proteins. $J$ Mol Biol 3:318-356.

Jacobs, E. 1997. Mycoplasma infections of the human respiratory tract. Wien Klin Wochenschr 109:574-577. 
Jadhav, V. R. and M. Yarus. 2002. Coenzymes as coribozymes. Biochimie 84:877-888.

Jaffe, J. D., H. C. Berg and G. M. Church. 2004. Proteogenomic mapping as a complementary method to perform genome annotation. Proteomics 4:59-77.

Jansen, J. A., T. J. McCarthy, G. A. Soukup and J. K. Soukup. 2006. Backbone and nucleobase contacts to glucosamine-6-phosphate in the $g \operatorname{lm} S$ ribozyme. Nat Struct Mol Biol 13:517-523.

Jault, J. M., S. Fieulaine, S. Nessler, P. Gonzalo, A. Di Pietro, J. Deutscher and A. Galinier. 2000. The HPr kinase from Bacillus subtilis is a homo-oligomeric enzyme which exhibits strong positive cooperativity for nucleotide and fructose-1,6-bisphosphate binding. $J$ Biol Chem 275:1773-1780.

Jeffares, D. C., A. M. Poole and D. Penny. 1998. Relics from the RNA world. J Mol Evol 46:18-36.

Jeffery, C. J. 1999. Moonlighting proteins. Trends Biochem Sci 24:8-11.

Jenal, U. and R. Hengge-Aronis. 2003. Regulation by proteolysis in bacterial cells. Curr Opin Microbiol 6:163-172.

Jin, S., M. De Jesus-Berrios and A. L. Sonenshein. 1996. A Bacillus subtilis malate dehydrogenase gene. J Bacteriol 178:560-563.

Johnston, W. K., P. J. Unrau, M. S. Lawrence, M. E. Glasner and D. P. Bartel. 2001. RNA-catalyzed RNA polymerization: accurate and general RNA-templated primer extension. Science 292:1319-1325.

Jones, B. E., V. Dossonnet, E. Küster, W. Hillen, J. Deutscher and R. E. Klevit. 1997. Binding of the catabolite repressor protein CcpA to its DNA target is regulated by phosphorylation of its corepressor HPr. J Biol Chem 272:26530-26535.

Jordan, S., A. Junker, J. D. Helmann and T. Mascher. 2006. Regulation of LiaRSdependent gene expression in Bacillus subtilis: identification of inhibitor proteins, regulator binding sites, and target genes of a conserved cell envelope stress-sensing two-component system. $J$ Bacteriol 188:5153-5166.

Jourlin-Castelli, C., N. Mani, M. M. Nakano and A. L. Sonenshein. 2000. CcpC, a novel regulator of the LysR family required for glucose repression of the citB gene in Bacillus subtilis. J Mol Biol 295:865-878.

Joyce, G. F. 1989. RNA evolution and the origins of life. Nature 338:217-224.

Joyce, G. F. 2002. The antiquity of RNA-based evolution. Nature 418:214-221. 
Kannan, T. R. and J. B. Baseman. 2000. Expression of UGA-containing Mycoplasma genes in Bacillus subtilis. J Bacteriol 182:2664-2667.

Kawamoto, H., T. Morita, A. Shimizu, T. Inada and H. Aiba. 2005. Implication of membrane localization of target mRNA in the action of a small RNA: mechanism of posttranscriptional regulation of glucose transporter in Escherichia coli. Genes Dev 19:328-338.

Kercher, M. A., P. Lu and M. Lewis. 1997. Lac repressor-operator complex. Curr Opin Struct Biol 7:76-85.

Kiefer, P., E. Heinzle, O. Zelder and C. Wittmann. 2004. Comparative metabolic flux analysis of lysine-producing Corynebacterium glutamicum cultured on glucose or fructose. Appl Environ Microbiol 70:229-239.

Kil, Y. V., V. N. Mironov, I. Gorishin, R. A. Kreneva and D. A. Perumov. 1992. Riboflavin operon of Bacillus subtilis: unusual symmetric arrangement of the regulatory region. Mol Gen Genet 233:483-486.

Kim, H. J., S. I. Kim, M. Ratnayake-Lecamwasam, K. Tachikawa, A. L. Sonenshein and M. Strauch. 2003a. Complex regulation of the Bacillus subtilis aconitase gene. J Bacteriol 185:1672-1680.

Kim, H. J., A. Roux and A. L. Sonenshein. 2002. Direct and indirect roles of CcpA in regulation of Bacillus subtilis Krebs cycle genes. Mol Microbiol 45:179-190.

Kim, J. H., Z. T. Guvener, J. Y. Cho, K. C. Chung and G. H. Chambliss. 1995. Specificity of DNA binding activity of the Bacillus subtilis catabolite control protein CcpA. $J$ Bacteriol 177:5129-5134.

Kim, S. I., C. Jourlin-Castelli, S. R. Wellington and A. L. Sonenshein. 2003b. Mechanism of repression by Bacillus subtilis CcpC, a LysR family regulator. J Mol Biol 334:609-624.

Kimata, K., T. Inada, H. Tagami and H. Aiba. 1998. A global repressor (Mlc) is involved in glucose induction of the pts $G$ gene encoding major glucose transporter in Escherichia coli. Mol Microbiol 29:1509-1519.

Kimata, K., Y. Tanaka, T. Inada and H. Aiba. 2001. Expression of the glucose transporter gene, pts $G$, is regulated at the mRNA degradation step in response to glycolytic flux in Escherichia coli. Embo J 20:3587-3595.

Knezevic, I., S. Bachem, A. Sickmann, H. E. Meyer, J. Stülke and W. Hengstenberg. 2000. Regulation of the glucose-specific phosphotransferase system (PTS) of Staphylococcus carnosus by the antiterminator protein GlcT. Microbiology 146:2333-2342.

Knudtson, K. L., M. Manohar, D. E. Joyner, E. A. Ahmed and B. C. Cole. 1997. Expression of the superantigen Mycoplasma arthritidis mitogen in Escherichia coli and characterization of the recombinant protein. Infect Immun 65:4965-4971. 
Koburger, T., J. Weibezahn, J. Bernhardt, G. Homuth and M. Hecker. 2005. Genomewide mRNA profiling in glucose starved Bacillus subtilis cells. Mol Genet Genomics 274:112.

Kochhar, S. and H. Paulus. 1996. Lysine-induced premature transcription termination in the lys $C$ operon of Bacillus subtilis. Microbiology 142:1635-1639.

Kraulis, P. J. 1991. MOLSCRIPT: a program to produce both detailed and schematic plots of protein structures. J Appl Crystallogr 24:946-950.

Krömer, J. O., O. Sorgenfrei, K. Klopprogge, E. Heinzle and C. Wittmann. 2004. Indepth profiling of lysine-producing Corynebacterium glutamicum by combined analysis of the transcriptome, metabolome, and fluxome. J Bacteriol 186:1769-1784.

Krüger, S., S. Gertz and M. Hecker. 1996. Transcriptional analysis of $b g l P H$ expression in Bacillus subtilis: evidence for two distinct pathways mediating carbon catabolite repression. $J$ Bacteriol 178:2637-2644.

Krüger, S. and M. Hecker. 1995. Regulation of the putative $b g l P H$ operon for aryl-ßglucoside utilization in Bacillus subtilis. J Bacteriol 177:5590-5597.

Kubodera, T., M. Watanabe, K. Yoshiuchi, N. Yamashita, A. Nishimura, S. Nakai, K. Gomi and H. Hanamoto. 2003. Thiamine-regulated gene expression of Aspergillus oryzae thiA requires splicing of the intron containing a riboswitch-like domain in the 5'-UTR. FEBS Lett 555:516-520.

Kumar, P. K., T. Kumarevel and H. Mizuno. 2006. Structural basis of HutP-mediated transcription anti-termination. Curr Opin Struct Biol 16:18-26.

Kunst, F., N. Ogasawara, I. Moszer, A. M. Albertini, G. Alloni, V. Azevedo, M. G. Bertero, P. Bessieres, A. Bolotin, S. Borchert, R. Borriss, L. Boursier, A. Brans, M. Braun, S. C. Brignell, S. Bron, S. Brouillet, C. V. Bruschi, B. Caldwell, V. Capuano, N. M. Carter, S. K. Choi, J. J. Codani, I. F. Connerton, A. Danchin and et al. 1997. The complete genome sequence of the gram-positive bacterium Bacillus subtilis. Nature 390:249256.

Kunst, F., M. Pascal, J. A. Lepesant, J. Walle and R. Dedonder. 1974. Purification and some properties of an endocellular sucrase from a constitutive mutant of Bacillus subtilis Marburg 168. Eur J Biochem 42:611-620.

Kunst, F. and G. Rapoport. 1995. Salt stress is an environmental signal affecting degradative enzyme synthesis in Bacillus subtilis. J Bacteriol 177:2403-2407.

Lai, E. C. 2003. RNA sensors and riboswitches: self-regulating messages. Curr Biol 13:R285-R291. 
Langbein, I., S. Bachem and J. Stülke. 1999. Specific interaction of the RNA-binding domain of the Bacillus subtilis transcriptional antiterminator GlcT with its RNA target, RAT. J Mol Biol 293:795-805.

Larralde, R., M. P. Robertson and S. L. Miller. 1995. Rates of decomposition of ribose and other sugars: implications for chemical evolution. Proc Natl Acad Sci USA 92:8158-8160.

Laursen, B. S., H. P. Sørensen, K. K. Mortensen and H. U. Sperling-Petersen. 2005. Initiation of protein synthesis in bacteria. Microbiol Mol Biol Rev 69:101-123.

Le Coq, D., C. Lindner, S. Krüger, M. Steinmetz and J. Stülke. 1995. New ß-glucoside (bgl) genes in Bacillus subtilis: the bglP gene product has both transport and regulatory functions similar to those of BglF, its Escherichia coli homolog. J Bacteriol 177:1527-1535.

Lee, J. M., S. Zhang, S. Saha, S. Santa Anna, C. Jiang and J. Perkins. 2001. RNA expression analysis using an antisense Bacillus subtilis genome array. J Bacteriol 183:73717380.

Lee, S. J., W. Boos, J. P. Bouche and J. Plumbridge. 2000. Signal transduction between a membrane-bound transporter, PtsG, and a soluble transcription factor, Mlc, of Escherichia coli. Embo J 19:5353-5361.

Legault, P., J. Li, J. Mogridge, K. E. Lewis and J. Greenblatt. 1998. NMR structure of the bacteriophage $\lambda \mathrm{N}$ peptide/boxB RNA complex: recognition of a GNRA fold by an argininerich motive. Cell 93:289-299.

Lenz, D. H., K. C. Mok, B. N. Lilley, R. V. Kulkarni, N. S. Wingreen and B. L. Bassler. 2004. The small RNA chaperone Hfq and multiple small RNAs control quorum sensing in Vibrio harveyi and Vibrio cholerae. Cell 118:69-82.

Leontis, N. B., J. Stombaugh and E. Westhof. 2002. The non-Watson-Crick base pairs and their associated isostericity matrices. Nucleic Acids Res 30:3497-3531.

Leontis, N. B. and E. Westhof. 2001. Geometric nomenclature and classification of RNA base pairs. $R N A$ 7:499-512.

Lescoute, A. and E. Westhof. 2005. Riboswitch structures: purine ligands replace tertiary contacts. Chem Biol 12:10-13.

Lévine, A., F. Vannier, C. Absalon, L. Kuhn, P. Jackson, E. Scrivener, V. Labas, J. Vinh, P. Courtney, J. Garin and S. J. Séror. 2006. Analysis of the dynamic Bacillus subtilis Ser/Thr/Tyr phosphoproteome implicated in a wide variety of cellular processes. Proteomics 6:2157-2173.

Levit, M. N., Y. Liu and J. B. Stock. 1998. Stimulus response coupling in bacterial chemotaxis: receptor dimers in signalling arrays. Mol Microbiol 30:459-466. 
Leyva-Vazquez, M. A. and P. Setlow. 1994. Cloning and nucleotide sequences of the genes encoding triose phosphate isomerase, phosphoglycerate mutase, and enolase from Bacillus subtilis. J Bacteriol 176:3903-3910.

Licht, A. and S. Brantl. 2006. Transcriptional repressor CcpN from Bacillus subtilis compensates asymmetric contact distribution by cooperative binding. J Mol Biol 364:434448.

Licht, A., S. Preis and S. Brantl. 2005. Implication of CcpN in the regulation of a novel untranslated RNA (SR1) in Bacillus subtilis. Mol Microbiol 58:189-206.

Lillo, F., S. Basile and R. N. Mantegna. 2002. Comparative genomics study of inverted repeats in bacteria. Bioinformatics 18:971-979.

Lind, K. 1983. Manifestations and complications of Mycoplasma pneumoniae disease: a review. Yale J Biol Med 56:461-468.

Lindner, C., A. Galinier, M. Hecker and J. Deutscher. 1999. Regulation of the activity of the Bacillus subtilis antiterminator LicT by multiple PEP-dependent, enzyme I- and HPrcatalysed phosphorylation. Mol Microbiol 31:995-1006.

Llanos, R. M., C. J. Harris, A. J. Hillier and B. E. Davidson. 1993. Identification of a novel operon in Lactococcus lactis encoding three enzymes for lactic acid synthesis: phosphofructokinase, pyruvate kinase, and lactate dehydrogenase. J Bacteriol 175:2541-2551.

Long, M. B., J. P. Jones, 3rd, B. A. Sullenger and J. Byun. 2003. Ribozyme-mediated revision of RNA and DNA. J Clin Invest 112:312-318.

Lu, Y., T. N. Shevtchenko and H. Paulus. 1992. Fine-structure mapping of cis-acting control sites in the lysC operon of Bacillus subtilis. FEMS Microbiol Lett 71:23-27.

Ludwig, H., G. Homuth, M. Schmalisch, F. M. Dyka, M. Hecker and J. Stülke. 2001. Transcription of glycolytic genes and operons in Bacillus subtilis: evidence for the presence of multiple levels of control of the gapA operon. Mol Microbiol 41:409-422.

Ludwig, H., C. Meinken, A. Matin and J. Stülke. 2002a. Insufficient expression of the ilvleu operon encoding enzymes of branched-chain amino acid biosynthesis limits growth of a Bacillus subtilis ccpA mutant. J Bacteriol 184:5174-5178.

Ludwig, H., N. Rebhan, H. M. Blencke, M. Merzbacher and J. Stülke. 2002b. Control of the glycolytic gapA operon by the catabolite control protein A in Bacillus subtilis: a novel mechanism of CcpA-mediated regulation. Mol Microbiol 45:543-553.

Luesink, E. J., R. E. van Herpen, B. P. Grossiord, O. P. Kuipers and W. M. de Vos. 1998. Transcriptional activation of the glycolytic las operon and catabolite repression of the gal operon in Lactococcus lactis are mediated by the catabolite control protein CcpA. Mol Microbiol 30:789-798. 
Mahadevan, S. and A. Wright. 1987. A bacterial gene involved in transcription antitermination: regulation at a rho-independent terminator in the $b g l$ operon of E. coli. Cell 50:485-494.

Majdalani, N., C. Cunning, D. Sledjeski, T. Elliott and S. Gottesman. 1998. DsrA RNA regulates translation of RpoS message by an anti-antisense mechanism, independent of its action as an antisilencer of transcription. Proc Natl Acad Sci USA 95:12462-12467.

Mandal, M., B. Boese, J. E. Barrick, W. C. Winkler and R. R. Breaker. 2003. Riboswitches control fundamental biochemical pathways in Bacillus subtilis and other bacteria. Cell 113:577-586.

Mandal, M. and R. R. Breaker. 2004. Adenine riboswitches and gene activation by disruption of a transcription terminator. Nat Struct Mol Biol 11:29-35.

Mandal, M., M. Lee, J. E. Barrick, Z. Weinberg, G. M. Emilsson, W. L. Ruzzo and R. R. Breaker. 2004. A glycine-dependent riboswitch that uses cooperative binding to control gene expression. Science 306:275-279.

Manival, X., Y. Yang, M. P. Strub, M. Kochoyan, M. Steinmetz and S. Aymerich. 1997. From genetic to structural characterization of a new class of RNA-binding domain within the SacY/BglG family of antiterminator proteins. EMBO J 16:5019-5029.

Martin-Verstraete, I., V. Charrier, J. Stülke, A. Galinier, B. Erni, G. Rapoport and J. Deutscher. 1998. Antagonistic effects of dual PTS-catalysed phosphorylation on the Bacillus subtilis transcriptional activator LevR. Mol Microbiol 28:293-303.

Martin-Verstraete, I., M. Débarbouillé, A. Klier and G. Rapoport. 1990. Levanase operon of Bacillus subtilis includes a fructose-specific phosphotransferase system regulating the expression of the operon. J Mol Biol 214:657-671.

Martin-Verstraete, I., M. Débarbouillé, A. Klier and G. Rapoport. 1992. Mutagenesis of the Bacillus subtilis "-12, -24" promoter of the levanase operon and evidence for the existence of an upstream activating sequence. J Mol Biol 226:85-99.

Massé, E., F. E. Escorcia and S. Gottesman. 2003. Coupled degradation of a small regulatory RNA and its mRNA targets in Escherichia coli. Genes Dev 17:2374-2383.

Massé, E., C. K. Vanderpool and S. Gottesman. 2005. Effect of RyhB small RNA on global iron use in Escherichia coli. J Bacteriol 187:6962-6971.

Mathews, D. H., M. D. Disney, J. L. Childs, S. J. Schroeder, M. Zuker and D. H. Turner. 2004. Incorporating chemical modification constraints into a dynamic programming algorithm for prediction of RNA secondary structure. Proc Natl Acad Sci USA 101:7287-7292.

Mathews, D. H., J. Sabina, M. Zuker and D. H. Turner. 1999. Expanded sequence dependence of thermodynamic parameters improves prediction of RNA secondary structure. $J$ Mol Biol 288:911-940. 
Mattick, J. S. and I. V. Makunin. 2005. Small regulatory RNAs in mammals. Hum Mol Genet 14 Spec No 1:R121-132.

Meadow, N. D., D. K. Fox and S. Roseman. 1990. The bacterial phosphoenolpyruvate: glycose phosphotransferase system. Annu Rev Biochem 59:497-542.

Meinken, C., H.-M. Blencke, H. Ludwig and J. Stülke. 2003. Expression of the glycolytic gapA operon in Bacillus subtilis: differential syntheses of proteins encoded by the operon. Microbiology 149:751-761.

Meinkoth, J. and G. Wahl. 1984. Hybridization of nucleic acids immobilized on solid supports. Anal Biochem 138:267-284.

Merianos, H. J., J. Wang and P. B. Moore. 2004. The structure of a ribosomal protein $\mathrm{S} 8 / \mathrm{spc}$ operon mRNA complex. RNA 10:954-964.

Miranda-Rios, J., C. Morera, H. Taboada, A. Davalos, S. Encarnacion, J. Mora and M. Soberon. 1997. Expression of thiamin biosynthetic genes (thiCOGE) and production of symbiotic terminal oxidase cbb3 in Rhizobium etli. J Bacteriol 179:6887-6893.

Miranda-Rios, J., M. Navarro and M. Soberon. 2001. A conserved RNA structure (thi box) is involved in regulation of thiamin biosynthetic gene expression in bacteria. Proc Natl Acad Sci USA 98:9736-9741.

Mironov, A. S., I. Gusarov, R. Rafikov, L. E. Lopez, K. Shatalin, R. A. Kreneva, D. A. Perumov and E. Nudler. 2002. Sensing small molecules by nascent RNA: a mechanism to control transcription in bacteria. Cell 111:747-756.

Mironov, V. N., A. S. Kraev, M. L. Chikindas, B. K. Chernov, A. I. Stepanov and K. G. Skryabin. 1994. Functional organization of the riboflavin biosynthesis operon from Bacillus subtilis SHgw. Mol Gen Genet 242:201-208.

Miwa, Y., A. Nakata, A. Ogiwara, M. Yamamoto and Y. Fujita. 2000. Evaluation and characterization of catabolite-responsive elements (cre) of Bacillus subtilis. Nucleic Acids Res 28:1206-1210.

Mizuno, T. 1997. Compilation of all genes encoding two-component phosphotransfer signal transducers in the genome of Escherichia coli. DNA Res 4:161-168.

Mizuno, T., M. Y. Chou and M. Inouye. 1984. A unique mechanism regulating gene expression: translational inhibition by a complementary RNA transcript (micRNA). Proc Natl Acad Sci USA 81:1966-1970.

Mogk, A., R. Hayward and W. Schumann. 1996. Integrative vectors for constructing single-copy transcriptional fusions between Bacillus subtilis promoters and various reporter genes encoding heat-stable enzymes. Gene 182:33-36. 
Moll, I., T. Afonyushkin, O. Vytvytska, V. R. Kaberdin and U. Blasi. 2003. Coincident Hfq binding and RNase E cleavage sites on mRNA and small regulatory RNAs. RNA 9:13081314.

Molle, V., Y. Nakaura, R. P. Shivers, H. Yamaguchi, R. Losick, Y. Fujita and A. L. Sonenshein. 2003. Additional targets of the Bacillus subtilis global regulator CodY identified by chromatin immunoprecipitation and genome-wide transcript analysis. $J$ Bacteriol 185:1911-1922.

Møller, T., T. Franch, P. Hojrup, D. R. Keene, H. P. Bachinger, R. G. Brennan and P. Valentin-Hansen. 2002. Hfq: a bacterial Sm-like protein that mediates RNA-RNA interaction. Mol Cell 9:23-30.

Moore, P. B. 1999. Structural motifs in RNA. Annu Rev Biochem 68:287-300.

Moreno, M. S., B. L. Schneider, R. R. Maile, W. Weyler and M. H. Saier, Jr. 2001. Catabolite repression mediated by the CcpA protein in Bacillus subtilis: novel modes of regulation revealed by whole-genome analyses. Mol Microbiol 39:1366-1381.

Morita, T., H. Kawamoto, T. Mizota, T. Inada and H. Aiba. 2004. Enolase in the RNA degradosome plays a crucial role in the rapid decay of glucose transporter mRNA in the response to phosphosugar stress in Escherichia coli. Mol Microbiol 54:1063-1075.

Müller, M. 2001. NMR-Untersuchungen an einer gefalteten RNA. Diplomarbeit, FriedrichAlexander-Universität Erlangen-Nürnberg.

Müller, M., J. E. Weigand, O. Weichenrieder and B. Suess. 2006. Thermodynamic characterization of an engineered tetracycline-binding riboswitch. Nucleic Acids Res 34:26072617.

Münchbach, M., A. Nocker and F. Narberhaus. 1999. Multiple small heat shock proteins in rhizobia. J Bacteriol 181:83-90.

Murphy, F. L. and T. R. Cech. 1994. GAAA tetraloop and conserved bulge stabilize tertiary structure of a group I intron domain. J Mol Biol 236:49-63.

Nagai, K. 1996. RNA-protein complexes. Curr Opin Struct Biol 6:53-61.

Narberhaus, F. 2002. mRNA-mediated detection of environmental conditions. Arch Microbiol 178:404-410.

Narberhaus, F., R. Käser, A. Nocker and H. Hennecke. 1998. A novel DNA element that controls bacterial heat shock gene expression. Mol Microbiol 28:315-323.

Narberhaus, F., T. Waldminghaus and S. Chowdhury. 2006. RNA thermometers. FEMS Microbiol Rev 30:3-16. 
Narberhaus, F., W. Weiglhofer, H. M. Fischer and H. Hennecke. 1996. The Bradyrhizobium japonicum rpoH $\mathrm{H}_{1}$ gene encoding a $\sigma^{32}$-like protein is part of a unique heat shock gene cluster together with groESL $L_{1}$ and three small heat shock genes. $J$ Bacteriol 178:5337-5346.

Neves, A. R., R. Ventura, N. Mansour, C. Shearman, M. J. Gasson, C. Maycock, A. Ramos and H. Santos. 2002. Is the glycolytic flux in Lactococcus lactis primarily controlled by the redox charge? Kinetics of NAD ${ }^{+}$and NADH pools determined in vivo by ${ }^{13} \mathrm{C}$ NMR. $J$ Biol Chem 277:28088-28098.

Niebisch, A., A. Kabus, C. Schultz, B. Weil and M. Bott. 2006. Corynebacterial protein kinase $\mathrm{G}$ controls 2-oxoglutarate dehydrogenase activity via the phosphorylation status of the OdhI protein. J Biol Chem 281:12300-12307.

Nielsen, J. 2003. It is all about metabolic fluxes. J Bacteriol 185:7031-7035.

Nobeli, I., R. A. Laskowski, W. S. Valdar and J. M. Thornton. 2001. On the molecular discrimination between adenine and guanine by proteins. Nucleic Acids Res 29:4294-4309.

Nocker, A., N. P. Krstulovic, X. Perret and F. Narberhaus. 2001. ROSE elements occur in disparate rhizobia and are functionally interchangeable between species. Arch Microbiol 176:44-51.

Noeske, J., C. Richter, M. A. Grundl, H. R. Nasiri, H. Schwalbe and J. Wöhnert. 2005. An intermolecular base triple as the basis of ligand specificity and affinity in the guanine- and adenine-sensing riboswitch RNAs. Proc Natl Acad Sci USA 102:1372-1377.

Noh, E. J., S. W. Kang, Y. J. Shin, D. C. Kim, I. S. Park, M. Y. Kim, B. G. Chun and B. H. Min. 2002. Characterization of Mycoplasma arginine deiminase expressed in E. coli and its inhibitory regulation of nitric oxide synthesis. Mol Cells 13:137-143.

Noller, H. F. 1993. Peptidyl transferase: protein, ribonucleoprotein, or RNA? J Bacteriol 175:5297-5300.

Nomura, M., J. L. Yates, D. Dean and L. E. Post. 1980. Feedback regulation of ribosomal protein gene expression in Escherichia coli: structural homology of ribosomal RNA and ribosomal protein mRNA. Proc Natl Acad Sci USA 77:7084-7088.

Nudler, E. 2006. Flipping riboswitches. Cell 126:19-22.

Nudler, E. and A. S. Mironov. 2004. The riboswitch control of bacterial metabolism. Trends Biochem Sci 29:11-17.

Oda, M., N. Kobayashi, A. Ito, Y. Kurusu and K. Taira. 2000. cis-acting regulatory sequences for antitermination in the transcript of the Bacillus subtilis hut operon and histidine-dependent binding of HutP to the transcript containing the regulatory sequences. Mol Microbiol 35:1244-1254. 
Opdyke, J. A., J. G. Kang and G. Storz. 2004. GadY, a small-RNA regulator of acid response genes in Escherichia coli. J Bacteriol 186:6698-6705.

Orth, P., D. Schnappinger, W. Hillen, W. Saenger and W. Hinrichs. 2000. Structural basis of gene regulation by the tetracycline inducible Tet repressor-operator system. Nat Struct Biol 7:215-219.

Otridge, J. and P. Gollnick. 1993. MtrB from Bacillus subtilis binds specifically to trp leader RNA in a tryptophan-dependent manner. Proc Natl Acad Sci USA 90:128-132.

Pabo, C. O. and R. T. Sauer. 1984. Protein-DNA recognition. Annu Rev Biochem 53:293321.

Pané-Farré, J., R. J. Lewis and J. Stülke. 2005. The RsbRST stress module in bacteria: a signalling system that may interact with different output modules. J Mol Microbiol Biotechnol 9:65-76.

Petersen, L. A. and D. M. Downs. 1997. Identification and characterization of an operon in Salmonella typhimurium involved in thiamine biosynthesis. J Bacteriol 179:4894-4900.

Pfeiffer, T., S. Schuster and S. Bonhoeffer. 2001. Cooperation and competition in the evolution of ATP-producing pathways. Science 292:504-507.

Plumbridge, J. 2002. Regulation of gene expression in the PTS in Escherichia coli: the role and interactions of Mlc. Curr Opin Microbiol 5:187-193.

Pollack, J. D., M. A. Myers, T. Dandekar and R. Herrmann. 2002. Suspected utility of enzymes with multiple activities in the small genome Mycoplasma species: the replacement of the missing "household" nucleoside diphosphate kinase gene and activity by glycolytic kinases. OMICS 6:247-258.

Poolman, B. 1993. Energy transduction in lactic acid bacteria. FEMS Microbiol Rev 12:125147.

Postma, P. W., J. W. Lengeler and G. R. Jacobson. 1993. Phosphoenolpyruvate:carbohydrate phosphotransferase systems of bacteria. Microbiol Rev 57:543-594.

Presecan-Siedel, E., A. Galinier, R. Longin, J. Deutscher, A. Danchin, P. Glaser and I. Martin-Verstraete. 1999. Catabolite regulation of the pta gene as part of carbon flow pathways in Bacillus subtilis. J Bacteriol 181:6889-6897.

Putzer, H., C. Condon, D. Brechemier-Baey, R. Brito and M. Grunberg-Manago. 2002. Transfer RNA-mediated antitermination in vitro. Nucleic Acids Res 30:3026-3033.

Quinn, C. L., B. T. Stephenson and R. L. Switzer. 1991. Functional organization and nucleotide sequence of the Bacillus subtilis pyrimidine biosynthetic operon. $J$ Biol Chem 266:9113-9127. 
Ramos, J. L., M. Martinez-Bueno, A. J. Molina-Henares, W. Teran, K. Watanabe, X. Zhang, M. T. Gallegos, R. Brennan and R. Tobes. 2005. The TetR family of transcriptional repressors. Microbiol Mol Biol Rev 69:326-356.

Ramström, H., S. Sanglier, E. Leize-Wagner, C. Philippe, A. Van Dorsselaer and J. Haiech. 2003. Properties and regulation of the bifunctional enzyme HPr kinase/phosphatase in Bacillus subtilis. J Biol Chem 278:1174-1185.

Reents, H., R. Münch, T. Dammeyer, D. Jahn and E. Härtig. 2006. The Fnr regulon of Bacillus subtilis. J Bacteriol 188:1103-1112.

Reichow, S. and G. Varani. 2006. Structural biology: RNA switches function. Nature 441:1054-1055.

Reizer, J., C. Hoischen, F. Titgemeyer, C. Rivolta, R. Rabus, J. Stülke, D. Karamata, M. H. Saier, Jr. and W. Hillen. 1998. A novel protein kinase that controls carbon catabolite repression in bacteria. Mol Microbiol 27:1157-1169.

Reizer, J., M. H. Saier, Jr., J. Deutscher, F. Grenier, J. Thompson and W. Hengstenberg. 1988. The phosphoenolpyruvate:sugar phosphotransferase system in grampositive bacteria: properties, mechanism, and regulation. Crit Rev Microbiol 15:297-338.

Renna, M. C., N. Najimudin, L. R. Winik and S. A. Zahler. 1993. Regulation of the Bacillus subtilis als $S$, alsD, and alsR genes involved in post-exponential-phase production of acetoin. J Bacteriol 175:3863-3875.

Repoila, F. and S. Gottesman. 2003. Temperature sensing by the $d s r A$ promoter. $J$ Bacteriol 185:6609-6614.

Rodionov, D. A., A. G. Vitreschak, A. A. Mironov and M. S. Gelfand. 2002. Comparative genomics of thiamin biosynthesis in procaryotes. New genes and regulatory mechanisms. $J$ Biol Chem 277:48949-48959.

Romby, P., F. Vandenesch and E. G. Wagner. 2006. The role of RNAs in the regulation of virulence-gene expression. Curr Opin Microbiol 9:229-236.

Rosenkrantz, M. S., D. W. Dingman and A. L. Sonenshein. 1985. Bacillus subtilis citB gene is regulated synergistically by glucose and glutamine. J Bacteriol 164:155-164.

Rutberg, B. 1997. Antitermination of transcription of catabolic operons. Mol Microbiol 23:413-421.

Saenger, W., P. Orth, C. Kisker, W. Hillen and W. Hinrichs. 2000. The Tetracycline Repressor-A Paradigm for a Biological Switch. Angew Chem Int Ed Engl 39:2042-2052. 
Saier, M. H., Jr., S. R. Goldman, R. R. Maile, M. S. Moreno, W. Weyler, N. Yang and I. T. Paulsen. 2002. Transport capabilities encoded within the Bacillus subtilis genome. J Mol Microbiol Biotechnol 4:37-67.

Sambrook, J., E. F. Fritsch and T. Maniatis. 1989. Molecular cloning: A laboratory manual. Cold Spring Harbor Laboratory Press, Cold Spring Harbor, N.Y.

Sarsero, J. P., E. Merino and C. Yanofsky. 2000. A Bacillus subtilis operon containing genes of unknown function senses tRNATrp charging and regulates expression of the genes of tryptophan biosynthesis. Proc Natl Acad Sci USA 97:2656-2661.

Sauer, U. 2004. High-throughput phenomics: experimental methods for mapping fluxomes. Curr Opin Biotechnol 15:58-63.

Sauer, U. and B. J. Eikmanns. 2005. The PEP-pyruvate-oxaloacetate node as the switch point for carbon flux distribution in bacteria. FEMS Microbiol Rev 29:765-794.

Sauer, U., V. Hatzimanikatis, J. E. Bailey, M. Hochuli, T. Szyperski and K. Wüthrich. 1997. Metabolic fluxes in riboflavin-producing Bacillus subtilis. Nat Biotechnol 15:448-452.

Sauer, U., V. Hatzimanikatis, H. P. Hohmann, M. Manneberg, A. P. van Loon and J. E. Bailey. 1996. Physiology and metabolic fluxes of wild-type and riboflavin-producing Bacillus subtilis. Appl Environ Microbiol 62:3687-3696.

Schägger, H. and G. von Jagow. 1987. Tricine-sodium dodecyl sulfate-polyacrylamide gel electrophoresis for the separation of proteins in the range from 1 to $100 \mathrm{kDa}$. Anal Biochem 166:368-379.

Schell, M. A. 1993. Molecular biology of the LysR family of transcriptional regulators. Annu Rev Microbiol 47:597-626.

Schilling, O., O. Frick, C. Herzberg, A. Ehrenreich, E. Heinzle, C. Wittmann and J. Stülke. 2007. Transcriptional and Metabolic Responses of Bacillus subtilis to the Availability of Organic Acids: Transcription Regulation Is Important but Not Sufficient To Account for Metabolic Adaptation. Appl Environ Microbiol 73:499-507.

Schilling, O., C. Herzberg, T. Hertrich, H. Vorsmann, D. Jessen, S. Hübner, F. Titgemeyer and J. Stülke. 2006. Keeping signals straight in transcription regulation: specificity determinants for the interaction of a family of conserved bacterial RNA-protein couples. Nucleic Acids Res 34:6102-6115.

Schilling, O., I. Langbein, M. Müller, M. Schmalisch and J. Stülke. 2004. A proteindependent riboswitch controlling ptsGHI operon expression in Bacillus subtilis: RNA structure rather than sequence provides interaction specificity. Nucleic Acids Res 32:28532864. 
Schirmer, F., S. Ehrt and W. Hillen. 1997. Expression, inducer spectrum, domain structure, and function of MopR, the regulator of phenol degradation in Acinetobacter calcoaceticus NCIB8250. J Bacteriol 179:1329-1336.

Schmalisch, M. H., S. Bachem and J. Stülke. 2003. Control of the Bacillus subtilis antiterminator protein GlcT by phosphorylation: elucidation of the phosphorylation chain leading to inactivation of GlcT. J Biol Chem 278:51108-51115.

Schnetz, K. and B. Rak. 1990. Beta-glucoside permease represses the $b g l$ operon of Escherichia coli by phosphorylation of the antiterminator protein and also interacts with glucose-specific enzyme III, the key element in catabolite control. Proc Natl Acad Sci USA 87:5074-5078.

Schnetz, K., J. Stülke, S. Gertz, S. Krüger, M. Krieg, M. Hecker and B. Rak. 1996. LicT, a Bacillus subtilis transcriptional antiterminator protein of the $\mathrm{BglG}$ family. $J$ Bacteriol 178:1971-1979.

Schreier, H. J. 1993. Biosynthesis of glutamine and glutamate and the assimilation of ammonia. In: Bacillus subtilis and other gram-positive bacteria, biochemistry, physiology, and molecular genetics. A. L. Sonenshein, J. A. Hoch and R. Losick. (eds.), ASM press, Washington DC, pp. 281-298.

Schreier, H. J., S. W. Brown, K. D. Hirschi, J. F. Nomellini and A. L. Sonenshein. 1989. Regulation of Bacillus subtilis glutamine synthetase gene expression by the product of the glnR gene. J Mol Biol 210:51-63.

Schroeder, R., A. Barta and K. Semrad. 2004. Strategies for RNA folding and assembly. Nat Rev Mol Cell Biol 5:908-919.

Schumacher, M. A., K. Y. Choi, H. Zalkin and R. G. Brennan. 1994. Crystal structure of LacI member, PurR, bound to DNA: minor groove binding by alpha helices. Science 266:763770 .

Seetharaman, S., M. Zivarts, N. Sudarsan and R. R. Breaker. 2001. Immobilized RNA switches for the analysis of complex chemical and biological mixtures. Nat Biotechnol 19:336-341.

Serganov, A., A. Polonskaia, A. T. Phan, R. R. Breaker and D. J. Patel. 2006. Structural basis for gene regulation by a thiamine pyrophosphate-sensing riboswitch. Nature 441:11671171 .

Servant, P., D. Le Coq and S. Aymerich. 2005. CcpN (YqzB), a novel regulator for CcpAindependent catabolite repression of Bacillus subtilis gluconeogenic genes. Mol Microbiol 55:1435-1451.

Sewer, A., N. Paul, P. Landgraf, A. Aravin, S. Pfeffer, M. J. Brownstein, T. Tuschl, E. van Nimwegen and M. Zavolan. 2005. Identification of clustered microRNAs using an $a b$ initio prediction method. BMC Bioinformatics 6:267. 
Shevtsov, M. B., Y. Chen, P. Gollnick and A. A. Antson. 2005. Crystal structure of Bacillus subtilis anti-TRAP protein, an antagonist of TRAP/RNA interaction. Proc Natl Acad Sci USA 102:17600-17605.

Shin, B. S., S. K. Choi and S. H. Park. 1999. Regulation of the Bacillus subtilis phosphotransacetylase gene. J Biochem 126:333-339.

Shivers, R. P., S. S. Dineen and A. L. Sonenshein. 2006. Positive regulation of Bacillus subtilis ackA by CodY and CcpA: establishing a potential hierarchy in carbon flow. Mol Microbiol 62:811-822.

Shivers, R. P. and A. L. Sonenshein. 2004. Activation of the Bacillus subtilis global regulator CodY by direct interaction with branched-chain amino acids. Mol Microbiol 53:599-611.

Shivers, R. P. and A. L. Sonenshein. 2005. Bacillus subtilis ilvB operon: an intersection of global regulons. Mol Microbiol 56:1549-1559.

Silvaggi, J. M., J. B. Perkins and R. Losick. 2005. Small untranslated RNA antitoxin in Bacillus subtilis. J Bacteriol 187:6641-6650.

Silvaggi, J. M., J. B. Perkins and R. Losick. 2006. Genes for small, noncoding RNAs under sporulation control in Bacillus subtilis. J Bacteriol 188:532-541.

Simic, P., J. Willuhn, H. Sahm and L. Eggeling. 2002. Identification of glyA (encoding serine hydroxymethyltransferase) and its use together with the exporter ThrE to increase Lthreonine accumulation by Corynebacterium glutamicum. Appl Environ Microbiol 68:33213327.

Simoneau, P., C.-M. Li, S. Loechel, R. Wenzel, R. Herrmann and P.-C. Hu. 1993. Codon reading scheme in Mycoplasma pneumoniae revealed by the analysis of the complete set of tRNA genes. Nucleic Acids Res 21:4967-4974.

Simoni, R. D., M. Levinthal, F. D. Kundig, W. Kundig, B. Anderson, P. E. Hartman and S. Roseman. 1967. Genetic evidence for the role of a bacterial phosphotransferase system in sugar transport. Proc Natl Acad Sci USA 58:1963-1970.

Skarlatos, P. and M. K. Dahl. 1998. The glucose kinase of Bacillus subtilis. J Bacteriol 180:3222-3226.

Smiley, B. K. and F. C. Minion. 1993. Enhanced readthrough of opal (UGA) stop codons and production of Mycoplasma pneumoniae P1 epitopes in Escherichia coli. Gene 134:33-40.

Sonenshein, A. L. 2002. The Krebs citric acid cycle. In: Bacillus subtilis and its closest relatives: from genes to cells. A. L. Sonenshein, J. A. Hoch and R. Losick. (eds.), ASM Press, Washington, DC, pp. 151-162. 
Sonntag, K., L. Eggeling, A. A. De Graaf and H. Sahm. 1993. Flux partitioning in the split pathway of lysine synthesis in Corynebacterium glutamicum. Quantification by ${ }^{13} \mathrm{C}-$ and ${ }^{1} \mathrm{H}-$ NMR spectroscopy. Eur J Biochem 213:1325-1331.

Springer, M., C. Protier and M. Grunberg-Manago. 1997. RNA mimicry in the translational apparatus. In: RNA structure and function. R. W. Simons and M. GrunbergManago. (eds.), Cold Spring Harbor Laboratory Press, Cold Spring Harbor, N.Y., pp. 377414.

Stamburski, C., J. Renaudin and J. M. Bove. 1991. First step toward a virus-derived vector for gene cloning and expression in spiroplasmas, organisms which read UGA as a tryptophan codon: synthesis of chloramphenicol acetyltransferase in Spiroplasma citri. J Bacteriol 173:2225-2230.

Steinhauer, K., T. Jepp, W. Hillen and J. Stülke. 2002. A novel mode of control of Mycoplasma pneumoniae HPr kinase/phosphatase activity reflects its parasitic lifestyle. Microbiology 148:3277-3284.

Steinmetz, M., D. Le Coq and S. Aymerich. 1989. Induction of saccharolytic enzymes by sucrose in Bacillus subtilis: evidence for two partially interchangeable regulatory pathways. $J$ Bacteriol 171:1519-1523.

Steinmetz, M., D. Le Coq, S. Aymerich, G. Gonzy-Tréboul and P. Gay. 1985. The DNA sequence of the gene for the secreted Bacillus subtilis enzyme levansucrase and its genetic control sites. Mol Gen Genet 200:220-228.

Stelzl, U., J. M. Zengel, M. Tovbina, M. Walker, K. H. Nierhaus, L. Lindahl and D. J. Patel. 2003. RNA-structural mimicry in Escherichia coli ribosomal protein L4-dependent regulation of the S10 operon. J Biol Chem 278:28237-28245.

Stock, A. M., V. L. Robinson and P. N. Goudreau. 2000. Two-component signal transduction. Annu Rev Biochem 69:183-215.

Strauss, J. H. and E. G. Strauss. 1988. Evolution of RNA viruses. Annu Rev Microbiol 42:657-683.

Stuart, K. and A. K. Panigrahi. 2002. RNA editing: complexity and complications. Mol Microbiol 45:591-596.

Stucki, J. W. 1980. The optimal efficiency and the economic degrees of coupling of oxidative phosphorylation. Eur J Biochem 109:269-283.

Stülke, J. 2002. Control of transcription termination in bacteria by RNA-binding proteins that modulate RNA structures. Arch Microbiol 177:433-440.

Stülke, J., M. Arnaud, G. Rapoport and I. Martin-Verstraete. 1998. PRD - a protein domain involved in PTS-dependent induction and carbon catabolite repression of catabolic operons in bacteria. Mol Microbiol 28:865-874. 
Stülke, J. and W. Hillen. 2000. Regulation of carbon catabolism in Bacillus species. Annu Rev Microbiol 54:849-880.

Stülke, J., I. Martin-Verstraete, V. Charrier, A. Klier, J. Deutscher and G. Rapoport. 1995. The HPr protein of the phosphotransferase system links induction and catabolite repression of the Bacillus subtilis levanase operon. J Bacteriol 177:6928-6936.

Stülke, J., I. Martin-Verstraete, M. Zagorec, M. Rose, A. Klier and G. Rapoport. 1997. Induction of the Bacillus subtilis ptsGHI operon by glucose is controlled by a novel antiterminator, GlcT. Mol Microbiol 25:65-78.

Stülke, J. and M. H. Schmalisch. 2004. The bacterial phosphotransferase system: a perfect link of sugar transport and signal transduction. Topics Curr Genet 9:179-205.

Sudarsan, N., J. E. Barrick and R. R. Breaker. 2003a. Metabolite-binding RNA domains are present in the genes of eukaryotes. $R N A$ 9:644-647.

Sudarsan, N., J. K. Wickiser, S. Nakamura, M. S. Ebert and R. R. Breaker. 2003b. An mRNA structure in bacteria that controls gene expression by binding lysine. Genes Dev 17:2688-2697.

Suess, B., B. Fink, C. Berens, R. Stentz and W. Hillen. 2004. A theophylline responsive riboswitch based on helix slipping controls gene expression in vivo. Nucleic Acids Res 32:1610-1614.

Suess, B., S. Hanson, C. Berens, B. Fink, R. Schroeder and W. Hillen. 2003. Conditional gene expression by controlling translation with tetracycline-binding aptamers. Nucleic Acids Res 31:1853-1858.

Switzer, R. L., R. J. Turner and Y. Lu. 1999. Regulation of the Bacillus subtilis pyrimidine biosynthetic operon by transcriptional attenuation: control of gene expression by an mRNAbinding protein. Prog Nucleic Acid Res Mol Biol 62:329-367.

Sykes, M. T. and M. Levitt. 2005. Describing RNA structure by libraries of clustered nucleotide doublets. $J$ Mol Biol 351:26-38.

Tanaka, K., K. Kobayashi and N. Ogasawara. 2003. The Bacillus subtilis YufLM twocomponent system regulates the expression of the malate transporters MaeN (YufR) and YflS, and is essential for utilization of malate in minimal medium. Microbiology 149:2317-2329.

Tanaka, Y., F. Itoh, K. Kimata and H. Aiba. 2004. Membrane localization itself but not binding to IICB is directly responsible for the inactivation of the global repressor Mlc in Escherichia coli. Mol Microbiol 53:941-951.

Tanaka, Y., K. Kimata and H. Aiba. 2000. A novel regulatory role of glucose transporter of Escherichia coli: membrane sequestration of a global repressor Mlc. Embo J 19:5344-5352. 
Tanaka, Y., K. Kimata, T. Inada, H. Tagami and H. Aiba. 1999. Negative regulation of the pts operon by Mlc: mechanism underlying glucose induction in Escherichia coli. Genes Cells 4:391-399.

Thore, S., M. Leibundgut and N. Ban. 2006. Structure of the eukaryotic thiamine pyrophosphate riboswitch with its regulatory ligand. Science 312:1208-1211.

Tobisch, S., J. Stülke and M. Hecker. 1999a. Regulation of the lic operon of Bacillus subtilis and characterization of potential phosphorylation sites of the LicR regulator protein by site-directed mutagenesis. J Bacteriol 181:4995-5003.

Tobisch, S., D. Zühlke, J. Bernhardt, J. Stülke and M. Hecker. 1999b. Role of CcpA in regulation of the central pathways of carbon catabolism in Bacillus subtilis. $J$ Bacteriol 181:6996-7004.

Tojo, S., T. Satomura, K. Morisaki, J. Deutscher, K. Hirooka and Y. Fujita. 2005. Elaborate transcription regulation of the Bacillus subtilis ilv-leu operon involved in the biosynthesis of branched-chain amino acids through global regulators of CcpA, CodY and TnrA. Mol Microbiol 56:1560-1573.

Tomchick, D. R., R. J. Turner, R. L. Switzer and J. L. Smith. 1998. Adaptation of an enzyme to regulatory function: structure of Bacillus subtilis PyrR, a pyr RNA-binding attenuation protein and uracil phosphoribosyltransferase. Structure 6:337-350.

Tomizawa, J., T. Itoh, G. Selzer and T. Som. 1981. Inhibition of ColE1 RNA primer formation by a plasmid-specified small RNA. Proc Natl Acad Sci USA 78:1421-1425.

Tortosa, P., S. Aymerich, C. Lindner, M. H. Saier, Jr., J. Reizer and D. Le Coq. 1997. Multiple phosphorylation of SacY, a Bacillus subtilis transcriptional antiterminator negatively controlled by the phosphotransferase system. J Biol Chem 272:17230-17237.

Tortosa, P., N. Declerck, H. Dutartre, C. Lindner, J. Deutscher and D. Le Coq. 2001. Sites of positive and negative regulation in the Bacillus subtilis antiterminators LicT and SacY. Mol Microbiol 41:1381-1393.

Tozzi, M. G., M. Camici, L. Mascia, F. Sgarrella and P. L. Ipata. 2006. Pentose phosphates in nucleoside interconversion and catabolism. Febs $J$ 273:1089-1101.

Trach, K., D. Burbulys, M. Strauch, J. J. Wu, N. Dhillon, R. Jonas, C. Hanstein, P. Kallio, M. Perego, T. Bird, G. Spiegelman, C. Fogher and J. A. Hoch. 1991. Control of the initiation of sporulation in Bacillus subtilis by a phosphorelay. Res Microbiol 142:815823.

Trieu-Cuot, P. and P. Courvalin. 1983. Nucleotide sequence of the Streptococcus faecalis plasmid gene encoding the 3'5"-aminoglycoside phosphotransferase type III. Gene 23:331341. 
Turinsky, A. J., F. J. Grundy, J. H. Kim, G. H. Chambliss and T. M. Henkin. 1998. Transcriptional activation of the Bacillus subtilis ackA gene requires sequences upstream of the promoter. J Bacteriol 180:5961-5967.

Turinsky, A. J., T. R. Moir-Blais, F. J. Grundy and T. M. Henkin. 2000. Bacillus subtilis cсpA gene mutants specifically defective in activation of acetoin biosynthesis. $J$ Bacteriol 182:5611-5614.

Valadkhan, S. 2005. snRNAs as the catalysts of pre-mRNA splicing. Curr Opin Chem Biol 9:603-608.

Valbuzzi, A. and C. Yanofsky. 2001. Inhibition of the B. subtilis regulatory protein TRAP by the TRAP-inhibitory protein, AT. Science 293:2057-2059.

Valegård, K., J. B. Murray, N. J. Stonehouse, S. van den Worm, P. G. Stockley and L. Liljas. 1997. The three-dimensional structures of two complexes between recombinant MS2 capsids and RNA operator fragments reveal sequence-specific protein-RNA interactions. $J$ Mol Biol 270:724-738.

Valentin-Hansen, P., M. Eriksen and C. Udesen. 2004. The bacterial Sm-like protein Hfq: a key player in RNA transactions. Mol Microbiol 51:1525-1533.

van Dijken, J. P., R. A. Weusthuis and J. T. Pronk. 1993. Kinetics of growth and sugar consumption in yeasts. Antonie Van Leeuwenhoek 63:343-352.

van Tilbeurgh, H., D. Le Coq and N. Declerck. 2001. Crystal structure of an activated form of the PTS regulation domain from the LicT transcriptional antiterminator. EMBO J 20:37893799.

van Tilbeurgh, H., X. Manival, S. Aymerich, J. M. Lhoste, C. Dumas and M. Kochoyan. 1997. Crystal structure of a new RNA-binding domain from the antiterminator protein SacY of Bacillus subtilis. EMBO J 16:5030-5036.

Vanderpool, C. K. and S. Gottesman. 2004. Involvement of a novel transcriptional activator and small RNA in post-transcriptional regulation of the glucose phosphoenolpyruvate phosphotransferase system. Mol Microbiol 54:1076-1089.

Viana, R., G. Pérez-Martinez, J. Deutscher and V. Monedero. 2005. The glycolytic genes pfk and pyk from Lactobacillus casei are induced by sugars transported by the phosphoenolpyruvate:sugar phosphotransferase system and repressed by CcpA. Arch Microbiol 183:385-393.

Vitreschak, A. G., D. A. Rodionov, A. A. Mironov and M. S. Gelfand. 2002. Regulation of riboflavin biosynthesis and transport genes in bacteria by transcriptional and translational attenuation. Nucleic Acids Res 30:3141-3151. 
Vitreschak, A. G., D. A. Rodionov, A. A. Mironov and M. S. Gelfand. 2004. Riboswitches: the oldest mechanism for the regulation of gene expression? Trends Genet 20:44-50.

Wach, A. 1996. PCR-synthesis of marker cassettes with long flanking homology regions for gene disruptions in S. cerevisiae. Yeast 12:259-265.

Wacker, I., H. Ludwig, I. Reif, H.-M. Blencke, C. Detsch and J. Stülke. 2003. The regulatory link between carbon and nitrogen metabolism in Bacillus subtilis: regulation of the gltAB operon by the catabolite control protein CcpA. Microbiology 149:3001-3009.

Wagner, E. G. and K. Flärdh. 2002. Antisense RNAs everywhere? Trends Genet 18:223226.

Waites, K. B. and D. F. Talkington. 2004. Mycoplasma pneumoniae and its role as a human pathogen. Clin Microbiol Rev 17:697-728.

Waldminghaus, T., A. Fippinger, J. Alfsmann and F. Narberhaus. 2005. RNA thermometers are common in $\alpha$ - and $\gamma$-proteobacteria. Biol Chem 386:1279-1286.

Wasinger, V. C., J. D. Pollack and I. Humphery-Smith. 2000. The proteome of Mycoplasma genitalium. Chaps-soluble component. Eur J Biochem 267:1571-1582.

Watson, J. D. and F. H. C. Crick. 1953. Molecular structure of nucleic acids. Nature 4356:737-742.

Weickert, M. J. and S. Adhya. 1992. A family of bacterial regulators homologous to Gal and Lac repressors. J Biol Chem 267:15869-15874.

Weinrauch, Y., T. Msadek, F. Kunst and D. Dubnau. 1991. Sequence and properties of comQ, a new competence regulatory gene of Bacillus subtilis. J Bacteriol 173:5685-5693.

Werstuck, G. and M. R. Green. 1998. Controlling gene expression in living cells through small molecule-RNA interactions. Science 282:296-298.

Wiechert, W. 2001. ${ }^{13} \mathrm{C}$ metabolic flux analysis. Metab Eng 3:195-206.

Wilson, S. A. and R. E. Drew. 1995. Transcriptional analysis of the amidase operon from Pseudomonas aeruginosa. J Bacteriol 177:3052-3057.

Wilson, S. A., S. J. Wachira, R. E. Drew, D. Jones and L. H. Pearl. 1993. Antitermination of amidase expression in Pseudomonas aeruginosa is controlled by a novel cytoplasmic amide-binding protein. EMBO J 12:3637-3642.

Wilson, S. A., S. J. Wachira, R. A. Norman, L. H. Pearl and R. E. Drew. 1996. Transcription antitermination regulation of the Pseudomonas aeruginosa amidase operon. EMBO J 15:5907-5916. 
Winkler, W. C. 2005. Metabolic monitoring by bacterial mRNAs. Arch Microbiol 183:151159.

Winkler, W. C. and R. R. Breaker. 2003. Genetic control by metabolite-binding riboswitches. ChemBioChem 4:1024-1032.

Winkler, W. C. and R. R. Breaker. 2005. Regulation of bacterial gene expression by riboswitches. Anпu Rev Microbiol 59:487-517.

Winkler, W. C., S. Cohen-Chalamish and R. R. Breaker. 2002a. An mRNA structure that controls gene expression by binding FMN. Proc Natl Acad Sci USA 99:15908-15913.

Winkler, W. C., A. Nahvi and R. R. Breaker. 2002b. Thiamine derivatives bind messenger RNAs directly to regulate bacterial gene expression. Nature 419:890-891.

Winkler, W. C., A. Nahvi, A. Roth, J. A. Collins and R. R. Breaker. 2004. Control of gene expression by a natural metabolite-responsive ribozyme. Nature 428:281-286.

Winkler, W. C., A. Nahvi, N. Sudarsan, J. E. Barrick and R. R. Breaker. 2003. An mRNA structure that controls gene expression by binding S-adenosylmethionine. Nat Struct Biol 10:701-707.

Wise, J. A. 1993. Guides to the heart of the spliceosome. Science 262:1978-1979.

Wittmann, C., M. Hans and E. Heinzle. 2002. In vivo analysis of intracellular amino acid labelings by GC/MS. Anal Biochem 307:379-382.

Wittmann, C. and E. Heinzle. 2001. Application of MALDI-TOF MS to lysine-producing Corynebacterium glutamicum: a novel approach for metabolic flux analysis. Eur J Biochem 268:2441-2455.

Wittmann, C. and E. Heinzle. 2002. Genealogy profiling through strain improvement by using metabolic network analysis: metabolic flux genealogy of several generations of lysineproducing corynebacteria. Appl Environ Microbiol 68:5843-5859.

Wittmann, C., P. Kiefer and O. Zelder. 2004. Metabolic fluxes in Corynebacterium glutamicum during lysine production with sucrose as carbon source. Appl Environ Microbiol 70:7277-7287.

Wray, L. V., Jr. and S. H. Fisher. 1994. Analysis of Bacillus subtilis hut operon expression indicates that histidine-dependent induction is mediated primarily by transcriptional antitermination and that amino acid repression is mediated by two mechanisms: regulation of transcription initiation and inhibition of histidine transport. J Bacteriol 176:5466-5473.

Xu, P., M. Guo and B. A. Hay. 2004. MicroRNAs and the regulation of cell death. Trends Genet 20:617-624. 
Yakhnin, H., A. V. Yakhnin and P. Babitzke. 2006. The trp RNA-binding attenuation protein (TRAP) of Bacillus subtilis regulates translation initiation of $y c b K$, a gene encoding a putative efflux protein, by blocking ribosome binding. Mol Microbiol 61:1252-1266.

Yakhnin, H., H. Zhang, A. V. Yakhnin and P. Babitzke. 2004. The trp RNA-binding attenuation protein of Bacillus subtilis regulates translation of the tryptophan transport gene trpP (yhaG) by blocking ribosome binding. J Bacteriol 186:278-286.

Yamamoto, H., M. Murata and J. Sekiguchi. 2000. The CitST two-component system regulates the expression of the Mg-citrate transporter in Bacillus subtilis. Mol Microbiol 37:898-912.

Yamanaka, K., M. Mitta and M. Inouye. 1999. Mutation analysis of the 5' untranslated region of the cold shock cspA mRNA of Escherichia coli. J Bacteriol 181:6284-6291.

Yang, H., F. Jossinet, N. Leontis, L. Chen, J. Westbrook, H. Berman and E. Westhof. 2003. Tools for the automatic identification and classification of RNA base pairs. Nucleic Acids Res 31:3450-3460.

Yang, M., X. Chen, K. Militello, R. Hoffman, B. Fernandez, C. Baumann and P. Gollnick. 1997. Alanine-scanning mutagenesis of Bacillus subtilis trp RNA-binding attenuation protein (TRAP) reveals residues involved in tryptophan binding and RNA binding. J Mol Biol 270:696-710.

Yang, Y., N. Declerck, X. Manival, S. Aymerich and M. Kochoyan. 2002. Solution structure of the LicT-RNA antitermination complex: CAT clamping RAT. EMBO J 21:19871997.

Yang, Y. T., K. Y. San and G. N. Bennett. 1999. Redistribution of metabolic fluxes in Escherichia coli with fermentative lactate dehydrogenase overexpression and deletion. Metab Eng 1:141-152.

Ying, S. Y., D. C. Chang, J. D. Miller and S. L. Lin. 2006. The microRNA: overview of the RNA gene that modulates gene functions. Methods Mol Biol 342:1-18.

Yoshida, K., K. Kobayashi, Y. Miwa, C. M. Kang, M. Matsunaga, H. Yamaguchi, S. Tojo, M. Yamamoto, R. Nishi, N. Ogasawara, T. Nakayama and Y. Fujita. 2001. Combined transcriptome and proteome analysis as a powerful approach to study genes under glucose repression in Bacillus subtilis. Nucleic Acids Res 29:683-692.

Yoshida, M. 1972. Allosteric nature of thermostable phosphofructokinase from an extreme thermophilic bacterium. Biochemistry 11:1087-1093.

Youngman, P. 1990. Use of transposons and integrational vectors for mutagenesis and construction of gene fusions in Bacillus subtilis. In: Molecular Biological Methods for Bacillus. C. R. Harwood and S. M. Cutting. (eds.), John Wiley \& Sons, Chichester, United Kingdom, pp. 221-266. 
Yousef, M. R., F. J. Grundy and T. M. Henkin. 2003. tRNA requirements for $g l y Q S$ antitermination: a new twist on tRNA. RNA 9:1148-1156.

Zamboni, N., E. Fischer, D. Laudert, S. Aymerich, H. P. Hohmann and U. Sauer. 2004. The Bacillus subtilis yqjI gene encodes the NADP ${ }^{+}$-dependent 6-P-gluconate dehydrogenase in the pentose phosphate pathway. J Bacteriol 186:4528-4534.

Zapp, M. L. and M. R. Green. 1989. Sequence-specific RNA binding by the HIV-1 Rev protein. Nature 342:714-716.

Zengel, J. M. and L. Lindahl. 1994. Diverse mechanisms for regulating ribosomal protein synthesis in Escherichia coli. Prog Nucleic Acid Res Mol Biol 47:331-370.

Zhang, A., K. M. Wassarman, C. Rosenow, B. C. Tjaden, G. Storz and S. Gottesman. 2003. Global analysis of small RNA and mRNA targets of Hfq. Mol Microbiol 50:1111-1124.

Zhang, H. and R. L. Switzer. 2003. Transcriptional pausing in the Bacillus subtilis pyr operon in vitro: a role in transcriptional attenuation? J Bacteriol 185:4764-4771.

Zhang, Y. and J. Skolnick. 2004. Automated structure prediction of weakly homologous proteins on a genomic scale. Proc Natl Acad Sci USA 101:7594-7599.

Zorrilla, S., T. Doan, C. Alfonso, E. Margeat, A. Ortega, G. Rivas, S. Aymerich, C. Royer and N. Declerck. 2007. Inducer-Modulated Cooperative Binding of the Tetrameric CggR Repressor to Operator DNA. Biophys J 92:3215-3227.

Zuker, M. 2003. Mfold web server for nucleic acid folding and hybridization prediction. Nucleic Acids Res 31:3406-3415. 


\section{Supplementary material}

\section{Oligonucleotides}

Table 13. Oligonucleotides

\begin{tabular}{|c|c|c|}
\hline Name & Sequence 5 ' $\rightarrow 3^{\prime}$ \# & Description \\
\hline cat-fwd & CGGCAATAGTTACCCTTATTATCAAG & $\begin{array}{l}\text { LFH-PCR }{ }^{*} \text {; amplification of } c a t \\
\text { cassette }\end{array}$ \\
\hline cat-rev & CCAGCGTGGACCGGCGAGGCTAGTTACCC & $\begin{array}{l}\text { LFH-PCR }{ }^{*} \text {; amplification of } c a t \\
\text { cassette }\end{array}$ \\
\hline $\mathrm{CH} 7$ & $\begin{array}{l}\text { AAAAGTCGACATGGATCTAAAACAACAAT } \\
\text { ACATTCTTG }\end{array}$ & $\begin{array}{l}\text { cloning of M. pneumoniae glpK gene } \\
\text { fw (SalI) }\end{array}$ \\
\hline $\mathrm{CH} 8$ & $\begin{array}{l}\text { TATAAAGCTTGTCTTAGTCTAAGCTAGCCC } \\
\text { ATTTTAG }\end{array}$ & $\begin{array}{l}\text { cloning of } M \text {. pneumoniae glpK gene } \\
\operatorname{rev}(\text { HindIII), A1512 } \rightarrow \mathrm{G}\end{array}$ \\
\hline $\mathrm{CH} 9$ & AAAAGTCGACATGGATCTAAAACAAC & $\begin{array}{l}\text { cloning of M. pneumoniae glpK gene } \\
\text { fw (SalI) }\end{array}$ \\
\hline $\mathrm{CH} 10$ & TATAAAGCTTGTCTTAGTCTAAGCTAG & $\begin{array}{l}\text { cloning of } M \text {. pneumoniae glpK gene } \\
\text { rev (HindIII) }\end{array}$ \\
\hline $\mathrm{CH} 11$ & $\begin{array}{l}\text { P-GATCCCTTAGAAATTTGGTCAGTCCAAT } \\
\text { TAG }\end{array}$ & $\begin{array}{l}\text { MMR: M. pneumoniae glpK } \\
\text { A165 } \rightarrow \mathrm{G}\end{array}$ \\
\hline $\mathrm{CH} 12$ & $\begin{array}{l}\text { P-CCATTGTGTTATGGAACAAAGAAAATGG } \\
\text { TTTG }\end{array}$ & $\begin{array}{l}\text { MMR: M. pneumoniae glpK } \\
\text { A273 } \rightarrow \mathrm{G}\end{array}$ \\
\hline $\mathrm{CH} 13$ & $\begin{array}{l}\text { P-CACTAAGATTGCTTGGATCTTGGAAAAT } \\
\text { GTTC }\end{array}$ & $\begin{array}{l}\text { MMR: M. pneumoniae glpK } \\
\text { A438 } \rightarrow \mathrm{G}\end{array}$ \\
\hline $\mathrm{CH} 14$ & P-CCTGGTTAATTTGGAAACTAACGGGTG & $\begin{array}{l}\text { MMR: M. pneumoniae glpK } \\
\text { A522 } \rightarrow \mathrm{G}\end{array}$ \\
\hline $\mathrm{CH} 15$ & P-CCATGACATGGTCACAAGAGTTAGGC & $\begin{array}{l}\text { MMR: M. pneumoniae glpK } \\
\text { A606 } \rightarrow \mathrm{G}\end{array}$ \\
\hline $\mathrm{CH} 16$ & P-TACCGAGTCATTGGTCTACTAGTGC & $\begin{array}{l}\text { MMR: M. pneumoniae glpK } \\
\mathrm{A} 705 \rightarrow \mathrm{G}\end{array}$ \\
\hline $\mathrm{CH} 17$ & $\begin{array}{l}\text { P-CCTTAAAGTGGTTAAGGGATAGTCTTAA } \\
\text { GG }\end{array}$ & $\begin{array}{l}\text { MMR: M. pneumoniae glpK } \\
\text { A966 } \rightarrow \mathrm{G}\end{array}$ \\
\hline $\mathrm{CH} 18$ & $\begin{array}{l}\text { P-GCAGTTAATTATTGGAAGGACACTAAAC } \\
\text { AAC }\end{array}$ & $\begin{array}{l}\text { MMR: M. pneumoniae glpK } \\
\text { A1386 } \rightarrow \mathrm{G}\end{array}$ \\
\hline $\mathrm{CH} 19$ & P-GAAATCAAAGCGTTGGAACGAAGCTG & $\begin{array}{l}\text { MMR: M. pneumoniae glpK } \\
\text { A1482 } \rightarrow \mathrm{G}\end{array}$ \\
\hline IL5 & $\begin{array}{l}\text { AAACAATTGAGGCATATCAGATTTACAAT } \\
\text { AGAAAG }\end{array}$ & $\begin{array}{l}\text { amplification of } p t s G \text { upstream } \\
\text { region; }(M f e \mathrm{I})\end{array}$ \\
\hline IL59 & $\begin{array}{l}\text { CCAAGTAATACGACTCACTATAGGACGTGTT } \\
\text { ACTGATTCG }\end{array}$ & $\begin{array}{l}\text { pts } G \text {-PCR-fragment for in vitro- } \\
\text { transcription; includes T7-promoter }\end{array}$ \\
\hline IL60 & $\begin{array}{l}\text { CAAGAATTGGGACAACTCTTCTTCTCCTTT } \\
\text { TTTTTCCTCAATCACTCATGCC }\end{array}$ & $\begin{array}{l}\text { pts } G \text {-PCR-fragment for in vitro- } \\
\text { transcription }\end{array}$ \\
\hline
\end{tabular}




\begin{tabular}{|c|c|c|}
\hline Name & Sequence $5^{\prime} \rightarrow 3^{\prime}$ \# & Description \\
\hline JS11 & TTT $\underline{G G A T C C G C A A C T G G A A G C A T A A G C G C ~}$ & $\begin{array}{l}\text { amplification of } p t s G \text { upstream } \\
\text { region; }(\text { BamHI })\end{array}$ \\
\hline JS39 & TCTATCAACAGGAGTCCA & sequencing of pWH844 constructs \\
\hline $\begin{array}{l}\text { spec- } \\
\text { fwd }\end{array}$ & $\begin{array}{l}\text { GACTGGCTCGCTAATAACGTAACGTGACT } \\
\text { GGCAAGAG }\end{array}$ & $\begin{array}{l}\text { LFH-PCR }{ }^{*} \text {; amplification of } s p c \\
\text { cassette }\end{array}$ \\
\hline $\begin{array}{l}\text { spec- } \\
\text { rev }\end{array}$ & $\begin{array}{l}\text { CGTAGCGAGGGCAAGGGTTTATTGTTTTCT } \\
\text { AAAATCTG }\end{array}$ & $\begin{array}{l}\text { LFH-PCR }{ }^{*} \text {; amplification of } s p c \\
\text { cassette }\end{array}$ \\
\hline OS25 & $\begin{array}{l}C C A A G T A A T A C G A C T C A C T A T A G G A A T T C A G \\
\text { TTTATCCTTAT }\end{array}$ & $\begin{array}{l}\text { ptsG-PCR-fragment for in vitro- } \\
\text { transcription of mutant RATs; } \\
\text { includes T7-promoter }\end{array}$ \\
\hline OS26 & TTGAGGGAAAAAA ACGGGAAGTTC & $\begin{array}{l}\text { pts } G \text {-PCR-fragment for in vitro- } \\
\text { transcription of mutant RATs }\end{array}$ \\
\hline OS28 & P-TTATCCTTATAACGATGTTACTGATTCG & mutagenesis of $p t s G$-RAT: A4ins \\
\hline OS29 & P-TCGATCAGGCAGAGTGATTGAGGGA & mutagenesis of $p t s G$-RAT: U25del \\
\hline OS30 & P-GTTTATCCTTATACCGTTGTTACTG & $\begin{array}{l}\text { mutagenesis of } p t s G \text {-RAT: } \mathrm{A} 1 \rightarrow \mathrm{C} \text {; } \\
\text { U4ins }\end{array}$ \\
\hline OS31 & P-CAGGCAAGAGGGATTGAGG & $\begin{array}{l}\text { mutagenesis of } p t s G \text {-RAT: } \mathrm{U} 25 \rightarrow \mathrm{A} \text {; } \\
\mathrm{U} 29 \rightarrow \mathrm{G}\end{array}$ \\
\hline OS32 & P-GCACCCAATTTTTCTCTTGCCTTTTTG & $\begin{array}{l}\text { mutagenesis of } p t s G \text {-RAT: terminator } \\
\text { region, } \mathrm{G} 84 \rightarrow \mathrm{U} ; \mathrm{A} 88 \rightarrow \mathrm{U}\end{array}$ \\
\hline OS33 & AAGGATCCCGTTTTATGAATGGCGGATG & sacT knockout; (BamHI) \\
\hline OS34 & TTTAAGCTTCATCTCGCTTTTGATCGAGC & sacT knockout; (HindIII) \\
\hline OS35 & $\begin{array}{l}\text { AAGGATCCGGCATTCCGATGATTTCAATC } \\
\mathrm{G}\end{array}$ & $\operatorname{sacY}$ knockout; (BamHI) \\
\hline OS36 & TTTAAGCTTGGAGAAAAGCCTTGGAACTC & sacY knockout; (HindIII) \\
\hline OS37 & CGTAATGCCTGTGGATAACG & sacT-forw1 (LFH-PCR ${ }^{*}$ for knockout) \\
\hline OS38 & $\begin{array}{l}\text { CCACGCTTACTACGTTGATAAGCCTATATG } \\
\text { CTCCTCCGGCAGTGTTTGC }\end{array}$ & $\begin{array}{l}\text { sacT-rev1 (LFH-PCR }{ }^{*} \text { for knockout); } \\
\text { complementary to tet-cassette }\end{array}$ \\
\hline OS39 & $\begin{array}{l}\text { GGGATCAACTTTGGGAGAGAGTTCGTTGG } \\
\text { AATCAAATGAAGCGCTCCATC }\end{array}$ & $\begin{array}{l}\text { sacT-forw2 (LFH-PCR }{ }^{*} \text { for } \\
\text { knockout); complementary to tet } \\
\text { cassette }\end{array}$ \\
\hline OS40 & CTTGCCTGCTTTACCAGTC & sacT-rev2 (LFH-PCR ${ }^{*}$ for knockout) \\
\hline OS41 & $\begin{array}{l}\text { CCTATCACCTCAAATGGTTCGCTGCTTTAT } \\
\text { TCATAGGGATGAGATC }\end{array}$ & $\begin{array}{l}\text { sacT-rev1 (LFH-PCR }{ }^{*} \text { for knockout); } \\
\text { complementary to kan cassette }\end{array}$ \\
\hline OS42 & $\begin{array}{l}\text { CGAGCGCCTACGAGGAATTTGTATCGCAT } \\
\text { TCGCCTATCAATGTGCG }\end{array}$ & $\begin{array}{l}s a c T \text {-forw2 (LFH-PCR }{ }^{*} \text { for } \\
\text { knockout); complementary to aphA3 } \\
\text { cassette }\end{array}$ \\
\hline OS43 & GGCAATAACAGCAAGCGG & $s a c Y$-forw1 (LFH-PCR ${ }^{*}$ for knockout) \\
\hline OS44 & $\begin{array}{l}\text { CTTGATAATAAGGGTAACTATTGCCGATC } \\
\text { CTTTACGACGATAGC }\end{array}$ & $\begin{array}{l}\text { sacY-rev1 (LFH-PCR }{ }^{*} \text { for knockout); } \\
\text { complementary to } c a t\end{array}$ \\
\hline
\end{tabular}




\begin{tabular}{|c|c|c|}
\hline Name & Sequence $5^{\prime} \rightarrow 3^{\prime}{ }^{\#}$ & Description \\
\hline OS45 & $\begin{array}{l}\text { GGGTAACTAGCCTCGCCGGTCCACGGCTA } \\
\text { AGCATCGGCACCTTTGTG }\end{array}$ & $\begin{array}{l}\text { sacY-forw2 (LFH-PCR }{ }^{*} \text { for } \\
\text { knockout); complementary to cat- } \\
\text { cassette }\end{array}$ \\
\hline OS46 & CGGTTCCTCTCTCTTAGATG & $s a c Y$-rev2 (LFH-PCR ${ }^{*}$ for knockout) \\
\hline OS47 & P-GCGCGGGGTGTTACTGATAAAG & $\begin{array}{l}\text { mutagenesis of } s a c B \text {-RAT: } \\
\mathrm{U} 4 \text {-deletion }+\mathrm{U} 3 \rightarrow \mathrm{G}\end{array}$ \\
\hline OS48 & P-GCAGGCATGACCTAAAATGTG & $\begin{array}{l}\text { mutagenesis of } s a c B \text {-RAT: } \\
\text { A24 } \rightarrow \text { U24 (U4del) }\end{array}$ \\
\hline OS49 & $\begin{array}{l}\text { AAAGAATTCGATCCTTTTTAACCCATCACA } \\
\text { TATAC }\end{array}$ & $\begin{array}{l}\text { amplification of } s a c B \text { upstream } \\
\text { region; }(E c o R)\end{array}$ \\
\hline OS50 & $\begin{array}{l}\text { TTTGGATCCTTTTTGATGTTCATCGTTCAT } \\
\text { GTC }\end{array}$ & $\begin{array}{l}\text { amplification of } s a c B \text { upstream } \\
\text { region; (BamHI) }\end{array}$ \\
\hline OS52 & P-GCACCCAATTTTCCTCTTGCCTTTTTG & $\begin{array}{l}\text { mutagenesis of } p t s G \text {-terminator: } \\
\mathrm{G} 84 \rightarrow \mathrm{C} ; \mathrm{A} 88 \rightarrow \mathrm{U}\end{array}$ \\
\hline OS53 & P-GTTTATCCTTATACCTTTGTTACTG & $\begin{array}{l}\text { mutagenesis of } p t s G \text {-RAT: } \mathrm{A} 1 \rightarrow \mathrm{C} \text {; } \\
\mathrm{G} 3 \rightarrow \mathrm{U} \text {; U4ins }\end{array}$ \\
\hline OS54 & P-CATATTTTAGGTCATTTTTTATTGTGCG & $\begin{array}{l}\text { mutagenesis of } s a c B \text {-RAT, terminator } \\
\text { region } \mathrm{U} 85 \rightarrow \mathrm{A}\end{array}$ \\
\hline OS55 & P-GCGCGGGTTGTTACTGATAAAG & $\begin{array}{l}\text { mutagenesis of } s a c B \text {-RAT: } \\
\text { U4-deletion }\end{array}$ \\
\hline OS56 & AAAGGATCCCACAATGGCTGGTGACGATC & amplification of $p d h B(B a m H \mathrm{I})$ \\
\hline OS57 & $\begin{array}{l}\text { AAAGGGCCCGGTGAAGGAATTTCTACTAC } \\
\text { TGC }\end{array}$ & amplification of $p d h B(A p a \mathrm{I})$ \\
\hline OS58 & $\begin{array}{l}\text { TTTAAGCTTCTAGGGTAAGTAAATTGAGT } \\
\text { A }\end{array}$ & amplification of $s p c$-cassette (HindIII) \\
\hline OS59 & TTTEAATTGATTGAGAGAAGTTTCTATAGA & amplification of spec-cassette ( $M f e \mathrm{I})$ \\
\hline OS60 & P-TATCCTTATAACTTTGTTACTGATTCG & $\begin{array}{l}\text { mutagenesis of } p t s G \text {-RAT (plasmid } \\
\text { pGP557 as template): RAT G3 } \rightarrow \mathrm{U}\end{array}$ \\
\hline OS61 & P-CGCGCGGGATTGTTACTG & mutagenesis of $s a c B$-RAT: $\mathrm{U} 3 \rightarrow \mathrm{A}$ \\
\hline OS62 & P-CGCGCGGGGTTGTTACTG & mutagenesis of $s a c B$-RAT: $\mathrm{U} 3 \rightarrow \mathrm{G}$ \\
\hline OS63 & GCTGATCAACTCCTGCGG & $\begin{array}{l}\text { knockout of } y d a P \text { with LFH-PCR } \\
\text { complementary to } y d a O\end{array}$ \\
\hline OS64 & $\begin{array}{l}\text { CCTATCACCTCAAATGGTTCGCTGCATTGC } \\
\text { TCAAGCAGCTCTGTC }\end{array}$ & $\begin{array}{l}\text { knockout of } y d a P \text { with LFH-PCR } \\
\text { complementary to } y d a P\end{array}$ \\
\hline OS65 & $\begin{array}{l}\text { CGAGCGCCTACGAGGAATTTGTATCGGAA } \\
\text { ACGAACCGCCGCTTC }\end{array}$ & $\begin{array}{l}\text { knockout of } y d a P \text { with LFH-PCR }{ }^{*} \text {; } \\
\text { complementary to } y d a P\end{array}$ \\
\hline OS66 & GATTGCAGGGGCTATTAATGC & $\begin{array}{l}\text { knockout of } y d a P \text { with LFH-PCR } \\
\text { complementary to } m n t H\end{array}$ \\
\hline OS67 & GGTTTAACAACGACGCCTGC & $\begin{array}{l}\text { knockout of } \operatorname{csr} A \text { with LFH-PCR } \\
\text { complementary to } y v i E\end{array}$ \\
\hline OS68 & $\begin{array}{l}\text { CCTATCACCTCAAATGGTTCGCTGGCACCT } \\
\text { ATTTGAATCGCTTCG }\end{array}$ & $\begin{array}{l}\text { knockout of } \operatorname{csr} A \text { with LFH-PCR } \\
\text { complementary to } \operatorname{csr} A\end{array}$ \\
\hline
\end{tabular}




\begin{tabular}{|c|c|c|}
\hline Name & Sequence $5^{\prime} \rightarrow 3^{\prime}$ \# & Description \\
\hline OS69 & $\begin{array}{l}\text { CGAGCGCCTACGAGGAATTTGTATCGCAG } \\
\text { GAAGAAAATAACCGTGCAG }\end{array}$ & $\begin{array}{l}\text { knockout of } \operatorname{csr} A \text { with LFH-PCR } \\
\text { complementary to } \operatorname{csr} A\end{array}$ \\
\hline OS70 & CTGTTGGTTTGCTTGAGCAAG & $\begin{array}{l}\text { knockout of } \operatorname{csr} A \text { with LFH-PCR } \\
\text { complementary to } h a g P\end{array}$ \\
\hline OS71 & P-GCAGGCAAAACCTAAAATGTG & mutagenesis of $s a c B$-RAT: $\mathrm{G} 26 \rightarrow \mathrm{A}$ \\
\hline OS72 & P-CATATTTTAGGTTTTTTTTTATTGTGCG & $\begin{array}{l}\text { mutagenesis of } s a c B \text {-terminator: } \\
\mathrm{C} 85 \rightarrow \mathrm{U}\end{array}$ \\
\hline OS73 & P-TTGTTACTGCGAAAGCAGGCAA & $\begin{array}{l}\text { mutagenesis of } s a c B \text {-RAT: } \\
\text { A13U14 } \rightarrow \text { CG }\end{array}$ \\
\hline OS74 & $\begin{array}{l}\text { AAAGGATCCGGAGGTGATAAGCCATGGCG } \\
\text { CAAATGACAATG }\end{array}$ & amplification of $p d h B(B a m H I)$ \\
\hline OS75 & $\begin{array}{l}\text { AAAGGATCCGCAGTTTCATTAAAATTCAA } \\
\text { GCAC }\end{array}$ & amplification of $p d h B(B a m H I)$ \\
\hline OS76 & TTGTTACTGCGAAAGCAGGCAAAACC & $\begin{array}{l}\text { mutagenesis of } s a c B-\mathrm{RAT}: \mathrm{G} 26 \rightarrow \mathrm{A} \\
\mathrm{A} 13 \mathrm{U} 14 \rightarrow \mathrm{CG}\end{array}$ \\
\hline OS77 & TTGTTACTGCGAAAGCAGGCAAGACC & $\begin{array}{l}\text { mutagenesis of } s a c B \text {-RAT: } \\
\text { A13U14 } \rightarrow \text { CG }\end{array}$ \\
\hline OS78 & $\begin{array}{l}\text { GATCTCTGGTTCCGCGTGGTTCCATGGGAT } \\
\text { CCGTCGACCTGCA }\end{array}$ & $\begin{array}{l}\text { introduction of thrombin restriction } \\
\text { site and MCS in pWH844 (BamHI / } \\
\text { PstI); complementary to OS79 }\end{array}$ \\
\hline OS79 & $\begin{array}{l}\text { GGTCGACGGATCCCATGGAACCACGCGGA } \\
\text { ACCAGA }\end{array}$ & see OS78 \\
\hline OS80 & $\begin{array}{l}\text { AAAGTCGACAAAATCTATAAAGTATTAAA } \\
\text { CAACAATGCG }\end{array}$ & cloning of SacT-RBD (forw); (SalI) \\
\hline OS81 & $\begin{array}{l}\text { TTTAAGCTTCTAGAACTTTTCATTCTCGTC } \\
\text { GCGCAC }\end{array}$ & cloning of SacT-RBD (rev); (HindIII) \\
\hline OS82 & $\begin{array}{l}\text { AAAGTCGACAAAATTGCGAAGGTGATCAA } \\
\text { CAATAATGTG }\end{array}$ & cloning of LicT-RBD (forw); (SalI) \\
\hline OS83 & $\begin{array}{l}\text { TTTAAGCTTCTATTCTGATACATCCTTGTT } \\
\text { ATCGAGC }\end{array}$ & cloning of LicT-RBD (rev); (HindIII) \\
\hline OS84 & $\begin{array}{l}\text { AAAGTCGACAAAATTAAAAGAATCTTAAA } \\
\text { TCATAATGCTATCG }\end{array}$ & cloning of SacY-RBD (forw); (SalI) \\
\hline OS85 & $\begin{array}{l}\text { TTTAAGCTTCTACTTATAGTCAGGTGTATC } \\
\text { TTTTCTG }\end{array}$ & cloning of SacY-RBD (rev); (HindIII) \\
\hline OS86 & $\begin{array}{l}\text { CCAAGTAATACGACTCACTATAGGCGAAAAG } \\
\text { TAAATCGCGCG }\end{array}$ & $\begin{array}{l}\text { sacB-PCR-fragment for in vitro- } \\
\text { transcription; includes T7-promoter }\end{array}$ \\
\hline OS87 & GTATACACTTTGCCCTTTACAC & $\begin{array}{l}\text { sacB-PCR-fragment for in vitro- } \\
\text { transcription }\end{array}$ \\
\hline OS88 & P-GGGTTTGTGACTGATAAAGCAGGC & mutagenesis of $s a c B$-RAT: $\mathrm{U} 8 \rightarrow \mathrm{G}$ \\
\hline OS89 & P-GGGATTGTGACTGATAAAGCAGGC & $\begin{array}{l}\text { mutagenesis of } s a c B \text {-RAT: } \mathrm{U} 8 \rightarrow \mathrm{G} \text {; } \\
\mathrm{U} 3 \rightarrow \mathrm{A}\end{array}$ \\
\hline
\end{tabular}




\begin{tabular}{|c|c|c|}
\hline Name & Sequence $5^{\prime} \rightarrow 3^{\prime}$, & Description \\
\hline OS90 & $\begin{array}{l}\text { P-CAGTTTATCCTTATAACGTTGTTACTGAT } \\
\text { AAAGCAGGCAAGAGTGATTGAGGG }\end{array}$ & $\begin{array}{l}\text { mutagenesis of } p t s G \text {-RAT mutates } \\
\text { top loop similar to } s a c B \text {-RAT }\end{array}$ \\
\hline OS91 & $\begin{array}{l}\text { TATGGAGCTCGGATCCTGGAGCCACCCGC } \\
\text { AGTTCGAAAAATGATAGT }\end{array}$ & $\begin{array}{l}\text { introduction of Strep-tag into pET3C, } \\
\text { complementary to OS92 }\end{array}$ \\
\hline OS92 & $\begin{array}{l}\text { GATCACTATCATTTTTCGAACTGCGGGTGG } \\
\text { CTCCAGGATCCGAGCTCCA }\end{array}$ & see OS91 \\
\hline OS93 & $\begin{array}{l}\text { AAACATATGGTGAATGGGTCCTTCACAGT } \\
\text { G }\end{array}$ & $\begin{array}{l}\text { cloning of GlcT-RBD into pGP574; } \\
(\text { Ndel })\end{array}$ \\
\hline OS94 & $\begin{array}{l}\text { AAAGGATCCTTGTTCCTTCTCGTCTTTTAA } \\
\text { AATGAAC }\end{array}$ & $\begin{array}{l}\text { cloning of GlcT-RBD into pGP574; } \\
(\text { BamHI })\end{array}$ \\
\hline OS95 & $\begin{array}{l}\text { AAACATATGAAAATTGCGAAGGTGATCAA } \\
\text { CAATAATGTG }\end{array}$ & $\begin{array}{l}\text { cloning of LicT-RBD into pGP574; } \\
(\text { Ndel) }\end{array}$ \\
\hline OS96 & $\begin{array}{l}\text { AAAGGATCCTGATACATCCTTGTTATCGA } \\
\text { GCG }\end{array}$ & $\begin{array}{l}\text { cloning of LicT-RBD into pGP574; } \\
(\text { BamHI) }\end{array}$ \\
\hline OS97 & $\begin{array}{l}\text { AAACATATGAAAATCTATAAAGTATTAAA } \\
\text { CAACAATGCG }\end{array}$ & $\begin{array}{l}\text { cloning of SacT-RBD into pGP574; } \\
(\text { Ndel })\end{array}$ \\
\hline OS98 & $\begin{array}{l}\text { AAAGGATCCGAACTTTTCATTCTCGTCGCG } \\
\text { CAC }\end{array}$ & $\begin{array}{l}\text { cloning of SacT-RBD into pGP574; } \\
(\text { BamHI })\end{array}$ \\
\hline OS99 & $\begin{array}{l}\text { AAACATATGAAAATTAAAAGAATCTTAAA } \\
\text { TCATAATGCTATCG }\end{array}$ & $\begin{array}{l}\text { cloning of SacY-RBD into pGP574; } \\
(\text { NdeI })\end{array}$ \\
\hline OS100 & $\begin{array}{l}\text { AAAGGATCCCTTATAGTCAGGTGTATCTTT } \\
\text { TCTG }\end{array}$ & $\begin{array}{l}\text { cloning of SacY-RBD into pGP574; } \\
\text { (BamHI) }\end{array}$ \\
\hline OS101 & NNNNNNNNN & nonamer random primer for labeling \\
\hline OS102 & P-GGATTGTGACTGGTAAAGCAGGCAAG & $\begin{array}{l}\text { mutagenesis of } s a c B \text {-RAT: } \mathrm{A} 13 \rightarrow \mathrm{G} \\
\mathrm{U} 8 \rightarrow \mathrm{G} ; \mathrm{U} 3 \rightarrow \mathrm{A}\end{array}$ \\
\hline OS103 & $\begin{array}{l}\text { P-CAGTTTATCCTTATAACATTGTGACTGA } \\
\text { TAAAGCAGGC }\end{array}$ & $\begin{array}{l}\text { mutagenesis of } p t s G \text {-RAT; mutates } \\
\text { top loop similar to } s a c B \text {-RAT } \\
+\mathrm{G} 3 \rightarrow \mathrm{A} ; \mathrm{U} 8 \rightarrow \mathrm{G}\end{array}$ \\
\hline OS104 & $\begin{array}{l}\text { P-CAGTTTATCCTTATAACTTTGTTACTGAT } \\
\text { AAAGCAGG }\end{array}$ & $\begin{array}{l}\text { mutagenesis of } p t s G \text {-RAT; mutates } \\
\text { top loop similar to } s a c B-\mathrm{RAT}+\mathrm{G} 3 \mathrm{U}\end{array}$ \\
\hline OS105 & P-GGTTTGTTACTGGTAAAGCAGGCAAG & mutagenesis of $s a c B$-RAT: $\mathrm{A} 13 \rightarrow \mathrm{G}$ \\
\hline OS106 & $\begin{array}{l}\text { AAAGGATCCTTATTCCGAGAGATCTTGAA } \\
\text { GAC }\end{array}$ & $\begin{array}{l}\text { cloning of SacT with C-terminal } \\
\text { Strep-tag }\end{array}$ \\
\hline OS107 & AAAGGATCCTCAGCGTGCGACTGACC & $\begin{array}{l}\text { cloning of SacY with C-terminal } \\
\text { Strep-tag }\end{array}$ \\
\hline OS108 & $\begin{array}{l}\text { AAAGGATCCTTCCGAGAGATCTTGAAGAC } \\
\text { G }\end{array}$ & $\begin{array}{l}\text { cloning of SacT-full with C-terminal } \\
\text { Strep-tag; }\end{array}$ \\
\hline OS109 & AAAGGATCCGCGTGCGACTGACCG & $\begin{array}{l}\text { cloning of SacY-full with C-terminal } \\
\text { Strep-tag. }\end{array}$ \\
\hline OS110 & TATCCTTATAACGTTGTTACTGATTCG & mutagenesis of $p t s G$-RAT: $\mathrm{U} 4_{\text {ins, }}$ \\
\hline OS111 & TATCCTTATAACATTGTTACTGATTCG & $\begin{array}{l}\text { mutagenesis of } p t s G \text {-RAT: } \mathrm{G} 3 \rightarrow \mathrm{A} \text {, } \\
\mathrm{U} 4_{\text {ins }}\end{array}$ \\
\hline
\end{tabular}




\begin{tabular}{|c|c|c|}
\hline Name & Sequence $5^{\prime} \rightarrow 3^{\prime}$ \# & Description \\
\hline OS112 & $\begin{array}{l}\text { CAGTTTATCCTTATAACAATGTTACTGATT } \\
\text { CG }\end{array}$ & $\begin{array}{l}\text { mutagenesis of } p t s G \text {-RAT: } \mathrm{A} 4_{\text {ins }}, \\
\mathrm{G} 3 \rightarrow \mathrm{A}\end{array}$ \\
\hline OS113 & CCAATTTTGCTCTGCCTTTTTGTTG & $\begin{array}{l}\text { mutagenesis of } p t s G \text {-RAT: terminator } \\
\text { region } \mathrm{A} 88_{\mathrm{del}}\end{array}$ \\
\hline OS114 & CGATCAGGCAGAGTGATTGAGG & mutagenesis of $p t s G$-RAT: $\mathrm{U} 25_{\mathrm{del}}$ \\
\hline OS115 & CCAATTTTGCTCATGTCCTTTTTGTTG & $\begin{array}{l}\text { mutagenesis of } p t s G \text {-RAT: terminator } \\
\text { region } \mathrm{T} 91_{\text {ins }}\end{array}$ \\
\hline OS116 & CTGATTCGATCAGGACATGAGTGATTG & mutagenesis of $p t s G$-RAT: $\mathrm{A}_{2} 3_{\text {ins }}$ \\
\hline OS117 & TGTTACTGATTCGAGCAGGCATGAGT & mutagenesis of $p t s G$-RAT: $\mathrm{U} 18 \rightarrow \mathrm{G}$ \\
\hline OS118 & CAGGCAAGAGTGATTGAGGGAA & mutagenesis of $p t s G$-RAT: $\mathrm{U} 25 \rightarrow \mathrm{A}$ \\
\hline OS119 & CCAATTTTGCTCTTGCCTTTTTGTTG & $\begin{array}{l}\text { mutagenesis of } p t s G \text {-RAT: terminator } \\
\text { region } \mathrm{A} 88 \rightarrow \mathrm{U}\end{array}$ \\
\hline SHU55 & GTTTTACAACGTCGTGACTGG & $\begin{array}{l}\text { template for RNA probe (E. coli } \\
\text { lacZ) }\end{array}$ \\
\hline SHU56 & $\begin{array}{l}\text { CTAATACGACTCACTATAGGGAGGTGTGCAG } \\
\text { TTCAACCACCG }\end{array}$ & $\begin{array}{l}\text { template for RNA probe }(E \text {. coli } \\
\text { lacZ); includes T7-promoter }\end{array}$ \\
\hline
\end{tabular}

Table 14. Oligonucleotides used for microarray analysis

\begin{tabular}{|c|c|c|}
\hline Name & $\begin{array}{l}\text { forward primer } \\
\text { sequence } 5^{\prime} \rightarrow 3^{\prime}\end{array}$ & $\begin{array}{l}\text { reverse primer } \\
\text { sequence } 5^{\prime} \rightarrow 3^{\prime} \text {, }\end{array}$ \\
\hline$p t s G$ & GGTTCGCGGAAAAATTCTCAATG & СTTCTTGTTCTCTGTTGACTGAAC \\
\hline$p t s H$ & $\begin{array}{l}\text { AAAGTCGACTGCATGGCACAAAAA } \\
\text { ACATTTAAAGTAACT (MS22) }\end{array}$ & $\begin{array}{l}\text { AAAGTCGACATGGCACAAAAAACA } \\
\text { TTTAAAGTAACT (MS25) }\end{array}$ \\
\hline$p t s I$ & TCGCGCGCTGCTTCGTG (AM4) & GTGTAATTAAGCGAAGGATTGCC \\
\hline pgi & $\begin{array}{l}\text { AAGGATCCATGACGCATGTACGCTT } \\
\text { GACTA }\end{array}$ & $\begin{array}{l}\text { TTTCTGCAGTTAATCTTCCAGACGT } \\
\text { TTTTCAAGC }\end{array}$ \\
\hline pgi-2 & CTTGACGGCTTGAACTATTTAGC & \\
\hline$p f k A$ & $\begin{array}{l}\text { AAGGATCCATGAAACGAATAGGGG } \\
\text { TATTAACG }\end{array}$ & $\begin{array}{l}\text { TTTCTGCAGTTAGATAGACAGTTCT } \\
\text { TTTGAAAGCTG }\end{array}$ \\
\hline pfk $A-2$ & $\begin{array}{l}\text { CACAGTATTATTATTGTTGCCGAAG } \\
\text { G }\end{array}$ & \\
\hline$f b a A$ & $\begin{array}{l}\text { AAGGATCCATGCCTTTAGTTTCTAT } \\
\text { GACG }\end{array}$ & $\begin{array}{l}\text { TTTCTGCAGTTAAGCTTGGTTTGAA } \\
\text { GAACCAAATTC }\end{array}$ \\
\hline$f b a A-2$ & CTTACAAAGGTGAGCCAAACCTTG & \\
\hline tpiA & $\begin{array}{l}\text { AAGGATCCATGAGAAAACCAATTA } \\
\text { TCGCCG }\end{array}$ & $\begin{array}{l}\text { TTTCTGCAGTTACTCATATTGACCTT } \\
\text { CCTCCA }\end{array}$ \\
\hline
\end{tabular}




\begin{tabular}{|c|c|c|}
\hline Name & $\begin{array}{l}\text { forward primer } \\
\text { sequence } 5^{\prime} \rightarrow 3, \#\end{array}$ & $\begin{array}{l}\text { reverse primer } \\
\text { sequence } 5^{\prime} \rightarrow 3\end{array}$ \\
\hline tpiA-2 & CTTGCTGGTCTTTCTGAAGAAC & \\
\hline gapA & CAATGCGTGTTCCAACTCCAAAC & $\begin{array}{l}\text { TTTAAGCTTTTAAAGACCTTTTTTTG } \\
\text { CGATGTAAGC }\end{array}$ \\
\hline gapA-2 & $\begin{array}{l}\text { AAAAGTCGACATGGCAGTAAAAGT } \\
\text { CGGTATTAACG }\end{array}$ & \\
\hline$p g k$ & $\begin{array}{l}\text { AAAAGTCGACAATAAAAAAACTCT } \\
\text { CAAAGACATCGACG }\end{array}$ & $\begin{array}{l}\text { TTTAAGCTTTTATTTATCGTTCAGTG } \\
\text { CAGCTAC }\end{array}$ \\
\hline$p g k-2$ & CAATCGACATTGGTACGAAAACAC & \\
\hline pgm & $\begin{array}{l}\text { AAGGATCCAGTAAAAAACCAGCTG } \\
\text { CACTCAT }\end{array}$ & $\begin{array}{l}\text { TTTCTGCAGTTATTTTTGAATTAAA } \\
\text { GATGTTCCTGTCATTTC }\end{array}$ \\
\hline pgm-2 & $\begin{array}{l}\text { GGAATGGTTGAACCAACAATTAAA } \\
\text { GC }\end{array}$ & \\
\hline eno & $\begin{array}{l}\text { AAGGATCCCCATACATTGTTGAT } \\
\text { GTTTATGCAC }\end{array}$ & $\begin{array}{l}\text { TTTCTGCAGTTACTTGTTTAAGTTGT } \\
\text { AGAAAGAGTTG }\end{array}$ \\
\hline eno-2 & GGTATTAAAAACGGCGTAGGCAAC & \\
\hline pyk & $\begin{array}{l}\text { AAGGATCCAGAAAAACTAAAATTG } \\
\text { TTTGTACCATCGG }\end{array}$ & $\begin{array}{l}\text { TTTCTGCAGTTAAAGAACGCTCGCA } \\
\text { CGG }\end{array}$ \\
\hline pyk-2 & CATTGGACGCAAATCAGCTTAC & \\
\hline$p d h A$ & $\begin{array}{l}\text { CTAATTGAAACACTTACATTCCGTT } \\
\text { ATG }\end{array}$ & TTACTTCGACTCCTTCTGTGTATA \\
\hline$p d h B$ & CATTAAAAGCTGCTGATGAGCTTG & $\begin{array}{l}\text { TTAAAATTCAAGCACTTTTCTTGCT } \\
\text { G }\end{array}$ \\
\hline$p d h C$ & $\begin{array}{l}\text { GAAATCAATGGCCTTGCAACAAAA } \\
\text { G }\end{array}$ & $\begin{array}{l}\text { TTACGCCTCCATTAAAATTAATTGT } \\
\text { GG }\end{array}$ \\
\hline$p d h D$ & GAACTTGCATCAGTTGGTTACAC & $\begin{array}{l}\text { TTATTTTACGATGTGAATCGGACTT } \\
\text { C }\end{array}$ \\
\hline als $S$ & $\begin{array}{l}\text { GTTTCTTATTCTCAGCAATGGAATT } \\
\text { AG }\end{array}$ & CTAGAGAGCTTTCGTTTTCATGAG \\
\hline als $S-2$ & CAAGGTGTCACACATGTATTTGG & CTACACTGTATTTTGTAATCGGC \\
\hline als $D$ & CAAGAAAAACCTTACGTGCCAATG & TTATTCAGGGCTTCCTTCAGTTG \\
\hline als $D-2$ & GTCAAAAAGATCAGCCTGTGAG & GCATAAAATAAGTTTCTGCTTGGC \\
\hline$l d h$ & $\begin{array}{l}\text { CAAATTGTAGATGATGTGAAAAAC } \\
\text { GC }\end{array}$ & $\begin{array}{l}\text { TTAGTTGACTTTTTGTTCTGCAAAA } \\
\text { TG }\end{array}$ \\
\hline pta & $\begin{array}{l}\text { CAGTGAAAATCGCGAAAGAAAAAG } \\
\text { C }\end{array}$ & TTACAGTGCTTGCGCCGCT \\
\hline ackA & $\begin{array}{l}\text { GTTGAAGCTACGAAAGAAGGAAAT } \\
\mathrm{G}\end{array}$ & TTATTTTGCTAAACGAACAACGTCG \\
\hline citA & CAGGGTGTACAAAACAAAAGACC & TTATGAAAGAACTTCCTCGGGAAT \\
\hline citZ & CGAAGCATCTGAAAGAAATGAGC & TTAGGCTCTTTCTTCAATCGGAA \\
\hline$i c d$ & GAGATTACATTTCTGATGCTCTTGC & $\begin{array}{l}\text { TTAGTCCATGTTTTTGATCAGTTCTT } \\
\text { C }\end{array}$ \\
\hline
\end{tabular}




\begin{tabular}{|c|c|c|}
\hline Name & $\begin{array}{l}\text { forward primer } \\
\text { sequence } 5, \rightarrow 3, \#\end{array}$ & $\begin{array}{l}\text { reverse primer } \\
\text { sequence } 5^{\prime} \rightarrow 3\end{array}$ \\
\hline$m d h$ & CTTGAAACTCTTATTCCGAAAGAAC & $\begin{array}{l}\text { TTAGGATAATACTTTCATGACATTT } \\
\text { TTGAC }\end{array}$ \\
\hline $\operatorname{cit} B$ & GCAAAAGGAACAAACCTTCTCG & TCAGGACTGCTTCATTTTTTCAC \\
\hline $\operatorname{cit} B-2$ & CAAGCGAGAAAAACGTTTACTAC & CCGCTTTATCTACCTGTACAG \\
\hline odhA & GGTAAAGTGTCTATAGACATTAGC & TTAGTTTTTGCGAGTCAAGCTATC \\
\hline$o d h B$ & CAGGAATCGAAAAAGAGATCGG & $\begin{array}{l}\text { TTATCCTTCTAATAAAAGCTGTTCA } \\
\text { GG }\end{array}$ \\
\hline sucC & CGATGGATATTATCAAGCATTATGG & $\begin{array}{l}\text { TTAAACTAAGGATACGATTTTCTGC } \\
\text { G }\end{array}$ \\
\hline sucC-2 & GCTTTTACAGCAGAAGAAGCAG & GTGTTTTCTCCGCAACCTCTTC \\
\hline sucD & GACGTTTTAAAAGCGTTTAACGAAG & TTAATGCGTTTTACAAGTTTCGAAC \\
\hline$s d h C$ & $\begin{array}{l}\text { GTCAATACAGCTACATGAGAAACT } \\
\text { G }\end{array}$ & $\begin{array}{l}\text { TTAAACAAATGCAAAAATCGCTTTT } \\
\text { AAGC }\end{array}$ \\
\hline $\operatorname{sdh} A$ & $\begin{array}{l}\text { GATGACAAAATTCAGGAGTTAATG } \\
\text { G }\end{array}$ & TTATTTCGCCACCTTCTTCTTC \\
\hline $\operatorname{sdh} B$ & GTCGTCAATGGGCATATGAATTATC & TTATACTCTGTCGCTTCCGAAG \\
\hline $\operatorname{cit} G$ & GAACTGAACGTGTTTAAGCCTG & TTACGCCTTTGGTTTTACCATG \\
\hline$z w f$ & $\begin{array}{l}\text { AAGGATCCGTGAAAACAAACCA } \\
\text { ACAACCAAAAG }\end{array}$ & TTATATGTTCCACCAGTGTAAGC \\
\hline$z w f-2$ & $\begin{array}{l}\text { CGCTTTACTTAAATGCTAAAAAGCT } \\
\text { TG }\end{array}$ & CCATTGCCAAGTAGAACATTCTG \\
\hline gnt $Z$ & $\begin{array}{l}\text { AAGGATCCTTCAATTCGATTGGTG } \\
\text { TCATAGG }\end{array}$ & $\begin{array}{l}\text { TTTCTGCAGTTATTCAGACCAATTC } \\
\text { GTATGGAAG }\end{array}$ \\
\hline gntZ-2 & CAAAGACATCGCCTTGATTTTCC & \\
\hline rpe & $\begin{array}{l}\text { AAAAGTCGACATAAAGGTTGCAC } \\
\text { CATCTATTCTTTC }\end{array}$ & $\begin{array}{l}\text { TTTAAGCTTTTATTTACTTCCTCTGA } \\
\text { TTTCAGAAATTG }\end{array}$ \\
\hline rpe-2 & CTCATTAAAGAGCAAGGAGTGAAG & \\
\hline$y w l F$ & $\begin{array}{l}\text { AAAAGTCGACAAAGTAGCCATTGC } \\
\text { ATCGGATC }\end{array}$ & $\begin{array}{l}\text { TTTAAGCTTCTACAGGTTTTTCTCTT } \\
\text { CATAATCG }\end{array}$ \\
\hline$y w l F-2$ & CGATTATCCGGATTATGCTTTTCC & GTTTGTGTCATTATGCTCCCTC \\
\hline$t k t$ & $\begin{array}{l}\text { AAGGATCCGATACAATTGAAAAG } \\
\text { AAATCAGTTGC }\end{array}$ & $\begin{array}{l}\text { CGAGTTAAGGCATTAATCAATAAGT } \\
\text { AAGCTTTTGAAA }\end{array}$ \\
\hline$t k t-2$ & GAGGTAGGTCTTGCAATTGAAG & $\begin{array}{l}\text { TTACTTATTGATTAATGCCTTAACT } \\
\text { CG }\end{array}$ \\
\hline$y w j H$ & $\begin{array}{l}\text { AAAAGTCGACATGTTATTCTTTGTT } \\
\text { GATACAGCCA }\end{array}$ & $\begin{array}{l}\text { TTTAAGCTTTTATTTGTTCCAGTCTG } \\
\text { CCAGG }\end{array}$ \\
\hline$y w j H-2$ & $\begin{array}{l}\text { GCATCAAAACAAACGTTACATTGAT } \\
\mathrm{C}\end{array}$ & \\
\hline$f b p$ & $\begin{array}{l}\text { AAGGATCCTTTAAAAATAATGTCAT } \\
\text { ACTTTTAAATTCACC }\end{array}$ & $\begin{array}{l}\text { TTTCTGCAGTCACTTCATATACCGA } \\
\text { TACTCC }\end{array}$ \\
\hline
\end{tabular}




\begin{tabular}{|c|c|c|}
\hline Name & $\begin{array}{l}\text { forward primer } \\
\text { sequence } 5^{\prime} \rightarrow 3, \#\end{array}$ & $\begin{array}{l}\text { reverse primer } \\
\text { sequence } 5^{\prime} \rightarrow 3\end{array}$ \\
\hline$f b p-2$ & $\begin{array}{l}\text { CATACACCTGTAAAAGAAATCGAA } \\
\text { G }\end{array}$ & CGCTTCTTTCGCATCAAAGTC \\
\hline $\operatorname{gap} B$ & $\begin{array}{l}\text { AAAAGTCGACAAGGTAAAAGTAGC } \\
\text { GATCAACG }\end{array}$ & $\begin{array}{l}\text { TTTAAGCTTTTATACAGCAGACGGA } \\
\text { TGTTTCA }\end{array}$ \\
\hline gap $B-2$ & GCATCTGAAAGGAAAACTTCACG & CCATCACAATGGTAACGTCTTC \\
\hline pckA & $\begin{array}{l}\text { AAAAGATCTAACTCAGTTGATTTGA } \\
\text { CCGCTG }\end{array}$ & $\begin{array}{l}\text { TTTCTGCAGTTATACGAGAGGGCCG } \\
\text { CCTG }\end{array}$ \\
\hline pckA-2 & CGTTTTCTTAGTCAATACCGGATG & CGCGCAAATAAATTGTGCCATG \\
\hline pycA & $\begin{array}{l}\text { AAAAGTCGACTTGTCTCAGCAATCG } \\
\text { ATACAAAAAG }\end{array}$ & $\begin{array}{l}\text { TTTTGTCGACTTATGCTTTTTCAATT } \\
\text { TCAAGGAGCAG }\end{array}$ \\
\hline pycA-2 & CGTCGTTTATTTCGAACTCAACG & GACGGCTTCAAGCGTTTCG \\
\hline glt $A$ & $\begin{array}{l}\text { AAGGATCCACGTACAATCAAATGC } \\
\text { CAAAAGCT }\end{array}$ & $\begin{array}{l}\text { TTTTGTCGACTTACTGTACTACCGC } \\
\text { TTGTTTTTG }\end{array}$ \\
\hline glt $A-2$ & GGTTTAAACGCAAATGCAACCTTG & CAAACGGACAGCTTTTTTGCG \\
\hline $\operatorname{glt} B$ & $\begin{array}{l}\text { AAGGATCCATGGGGAAACCAACTG } \\
\text { GATTTAT }\end{array}$ & $\begin{array}{l}\text { TTTCTGCAGTTACGGAAGAACTGAA } \\
\text { CTCCCA }\end{array}$ \\
\hline$g l t B-2$ & $\begin{array}{l}\text { CTTCATACCATCCAAATGGAAAAA } \\
\text { GTG }\end{array}$ & CTTTATCGATAATGGTCCGCTC \\
\hline$g \ln A$ & CAAAACAGAAGCCCGCTTATC & GAGACATATACTGTTCGCGTTC \\
\hline$n r g B$ & $\begin{array}{l}\text { AAACATATGAGCGGTCAAATGTTC } \\
\text { AAGGTA (CD35) }\end{array}$ & GATGTTGATCGTATTGCTGATTTC \\
\hline $\operatorname{roc} G$ & GATATCCCTTATTTGCTCGAT (PT10) & СTCTTCTTCCGACCAATAATATC \\
\hline $\operatorname{roc} G-2$ & GCAAAGCAAGTCTCGAAAGATG & CCGTAAGGAAGATTGGCAATC \\
\hline $\operatorname{roc} A$ & CAGATGTTGATGAAAACGCCC & AAAGCTTCGGACGTTGTTTTGG \\
\hline rocD & AGAATGTCTTGTTTATTGCGGATGA & TCTGCCGCGGACTTCTTTAAT \\
\hline $\operatorname{gudB}$ & CATAATATCCGGGCTAAAATTGTC & TATCCAGCCTCTAAAACGCGAA \\
\hline $\arg C$ & TTCGTTATCGTTGACGCGAAGA & ATTGCTGCCGTACACTTCTTTC \\
\hline $\arg G$ & ACTGGTGAAAGAGGTAGCC & GCTTTAATCTGCTCCTTCTTTTTC \\
\hline $\operatorname{car} A$ & $\begin{array}{l}\text { TAAACATATCGCTCTCATTGATTTT } \\
\text { GG }\end{array}$ & CCACATAGCTGTGGTTTTGG \\
\hline $\operatorname{asp} B$ & ACCCGACATCAATCGCACAATA & GCTATGTTTTTCTACAAAACGCTTG \\
\hline ans $A$ & CAGATGTATGTCTGCTGAAGC & CAGGACAACGTCATCAGCTAT \\
\hline pyrAA & AATGTGACGTCATCGTTGTG & TATGCGTCACTTCCAGTTCT \\
\hline$n r g A$ & ATGTACGCGTTCATCAACACAAA & TAAAATAACCCATCTGCGCCC \\
\hline$c i t M$ & $\begin{array}{l}\text { CTTTGGTGGTTCAATTTATTGTTAA } \\
\text { CG }\end{array}$ & GTCATTCGGCATCAAAAATGTG \\
\hline$c i t H$ & CACGTTCACTCATCCCTGAT & $\begin{array}{l}\text { GAAAGAAATAATCCCAATCAAAAG } \\
\text { CG }\end{array}$ \\
\hline $\operatorname{dct} P$ & CTTTACGGATTCAGCCTGTG & CGTTGAGGCAAGGACGATAAAT \\
\hline
\end{tabular}




\begin{tabular}{|c|c|c|}
\hline Name & $\begin{array}{l}\text { forward primer } \\
\text { sequence } 5^{\prime} \rightarrow 3,{ }^{\prime}\end{array}$ & $\begin{array}{l}\text { reverse primer } \\
\text { sequence } 5^{\prime} \rightarrow 3^{, \#}\end{array}$ \\
\hline gltP & CTCCGGCTTGTCATTGAACT & CTTTCAGTTTGGCTGTTTGCG \\
\hline glt $T$ & AATCTATTGCCGCACTCTTTTTAG & ACCAGAAATGGTTGCGTTTTGTT \\
\hline$g \ln Q$ & GCCATTAACGACAGAAAAACTGAT & CTAGCACCTCACCGATCATTT \\
\hline pps & CTATCTCCACCACGTGTTATC & CAATGTAGCCTTCTGTACCATG \\
\hline yqjI & CCAAATGAAAGCTGCTTCTGAAG & CCATTCAGTATGGAAGATGCC \\
\hline mleA & GCTGTATACGGAACAGACAAATC & GGACAACATCACCTTGTTTAGTG \\
\hline mals & CAACTCTTTCTGAAGCAAAGCC & CCTGTATAATGTCTTCAGGCTC \\
\hline$y d a P$ & GAACGCGAATCAGGATTTCATC & CAATCATGACATCCACTACGAC \\
\hline hns-1 & $\begin{array}{l}C C A A G T A A T A C G A C T C A C T A T A G G G A \\
\text { AACGCTGGAAGAAATGCTG }\end{array}$ & GCTTGATCAGGAAATCGTCGAG \\
\hline отрA-1 & $\begin{array}{l}C C A A G T A A T A C G A C T C A C T A T A G G C A \\
\text { ACTTCAACAAAGCAACCCTG }\end{array}$ & CTGAGTTACAACGTCTTTGATACC \\
\hline отрA-2 & $\begin{array}{l}C C A A G T A A T A C G A C T C A C T A T A G G C A \\
\text { TGACACTGGTTTCATCAAC }\end{array}$ & GTTGTTGGTCCACTGGTATTC \\
\hline отр C-1 & $\begin{array}{l}C C A A G T A A T A C G A C T C A C T A T A G G \mathrm{GA} \\
\text { AATACGACGCTAACAACATC }\end{array}$ & CGATGTTATCAGTGTTGATGCC \\
\hline ompC-2 & $\begin{array}{l}C C A A G T A A T A C G A C T C A C T A T A G G C A \\
\text { TTCGCAGGTCTGAAATTC }\end{array}$ & GTTCTGAGCATCAGTACGTTTG \\
\hline mpn474-1 & GACCAAACACAACTGCTTGAC & $\begin{array}{l}\text { CTAATACGACTCACTATAGGGAGACTT } \\
\text { GCAATTGTTG (SH75) }\end{array}$ \\
\hline mpn474-2 & $\begin{array}{l}C C A A G T A A T A C G A C T C A C T A T A G G C A \\
\text { GCAACAATTCCATCAAGC }\end{array}$ & GTAACAACTCGTAAGCGGAG \\
\hline mpn474-3 & $\begin{array}{l}\text { CCAAGTAATACGACTCACTATAGGC } \\
\text { CACTAACTTTGAGTTAGGTC }\end{array}$ & CATCACTCTGTTGCAAGTGG \\
\hline mpn474-4 & $\begin{array}{l}\text { ACTCCTTCAACCCAACAAGTC } \\
\text { (SH74) }\end{array}$ & GTAGTTGGTGCAACTGTGTC \\
\hline$m p n 474-5$ & CAACTCCAAGCTGCTTACAATAAC & GCTTGCCATTGATCTTAGCTAG \\
\hline
\end{tabular}

\# restriction sites are underlined, promoters are italic

\section{Plasmids}

Table 15. Plasmids used in this work

\begin{tabular}{llll}
\hline Plasmid & Resistance & Description & Reference \\
\hline pAC7 & $\begin{array}{l}\text { ampicillin/ } \\
\text { kanamycin }\end{array}$ & $\begin{array}{l}\text { construction of translational } \\
\text { lacZ fusions }\end{array}$ & Weinrauch et al., 1991 \\
pBgaB & $\begin{array}{l}\text { ampicillin/ } \\
\text { neomycin }\end{array}$ & $\begin{array}{l}\text { construction of translational } \\
\text { lacZ fusions with thermostable } \\
\beta \text {-galactosidase }\end{array}$ & Mogk et al., 1996 \\
& ampicillin & cloning & Stratagene, USA \\
pBluescript II & & &
\end{tabular}




\begin{tabular}{|c|c|c|c|}
\hline Plasmid & Resistance & Description & Reference \\
\hline pDG1726 & spectinomycin & PCR template for $s p c$-cassette & $\begin{array}{l}\text { Guérout-Fleury et al., } \\
1995\end{array}$ \\
\hline pET3C & ampicillin & $\begin{array}{l}\text { construction of overexpression } \\
\text { vector with C-terminal Strep- } \\
\text { tag (pGP574) }\end{array}$ & Novagen, Germany \\
\hline pGEM-cat & chloramphenicol & PCR template for cat-cassette & Youngman, 1990 \\
\hline pGP66 & $\begin{array}{l}\text { ampicillin/ } \\
\text { kanamycin }\end{array}$ & $\begin{array}{l}p t s G \text {-lacZ fusion: } \\
\text { wild type RAT }\end{array}$ & Stülke et al., 1997 \\
\hline pGP230 & ampicillin & overexpression of GlcT-RBD & Schilling et al., 2004 \\
\hline pGP253 & $\begin{array}{l}\mathrm{pWH} 844+g l p K \\
\text { SalI/HindIII }\end{array}$ & $\begin{array}{l}\text { PCR: } g p l K \mathrm{CH} 7+\mathrm{CH} 8 \text {; } \\
\mathrm{A} 1512 \rightarrow \mathrm{G}\end{array}$ & Hames et al., 2005 \\
\hline pGP254 & $\begin{array}{l}\text { pWH844 + glpK } \\
\text { SalI/HindIII }\end{array}$ & $\begin{array}{l}\text { PCR: } g p l K \mathrm{CH} 7+\mathrm{CH} 10 \text {; } \\
\text { all TGA codons converted } \\
\text { into TGG }\end{array}$ & Hames et al., 2005 \\
\hline pGP332 & $\begin{array}{l}\text { ampicillin/ } \\
\text { kanamycin }\end{array}$ & $\begin{array}{l}\text { ptsG-lacZ fusion: pAC6/MfeI/ } \\
\text { BamHI; terminator G37C G38C }\end{array}$ & Schilling et al., 2004 \\
\hline pGP339 & $\begin{array}{l}\text { ampicillin/ } \\
\text { chloramphenicol }\end{array}$ & $\begin{array}{l}\text { ptsG-lacZ fusion pAC7/BamHI } \\
\text { RAT G11A G26A }\end{array}$ & Schilling et al., 2004 \\
\hline pGP340 & $\begin{array}{l}\text { ampicillin/ } \\
\text { chloramphenicol }\end{array}$ & $\begin{array}{l}\text { pts G-lacZ fusion: pAC7/BamHI } \\
\text { RAT G11C G26A }\end{array}$ & Schilling et al., 2004 \\
\hline pGP342 & $\begin{array}{l}\text { ampicillin/ } \\
\text { chloramphenicol }\end{array}$ & $\begin{array}{l}\text { pts G-lacZ fusion: } \mathrm{pAC} 7 / \text { BamHI } \\
\text { wild type RAT }\end{array}$ & Schilling et al., 2004 \\
\hline pWH844 & ampicillin & overexpression vector & Schirmer et al., 1997 \\
\hline
\end{tabular}

Table 16. Plasmids constructed in this work

\begin{tabular}{|c|c|c|c|}
\hline Plasmid & Construction & Description & Reference \\
\hline pGP549 & $\begin{array}{l}\mathrm{pAC7} / \text { BamHI + } \\
\text { EcoRI }\end{array}$ & $\begin{array}{l}\text { CCR: ptsG-RAT IL5 + JS11 + OS28/ } \\
\text { BamHI + MfeI (ptsG-R1) }\end{array}$ & Schilling et al., 2004 \\
\hline pGP550 & $\begin{array}{l}\mathrm{pAC7} / \text { BamHI + } \\
\text { EcoRI }\end{array}$ & $\begin{array}{l}\text { MMR: ptsG-RAT IL5 + JS11 + } \\
\text { OS116 + OS115 / BamHI + MfeI } \\
(p t s G-R 2)\end{array}$ & Schilling et al., 2006 \\
\hline pGP551 & $\begin{array}{l}\text { pAC7 / BamHI + } \\
\text { EcoRI }\end{array}$ & $\begin{array}{l}\text { MMR: ptsG-RAT IL5 + JS11 + OS28 } \\
+ \text { OS117 / BamHI + MfeI }\end{array}$ & \\
\hline pGP552 & $\begin{array}{l}\mathrm{pAC7} / \text { BamHI + } \\
\text { EcoRI }\end{array}$ & $\begin{array}{l}\text { MMR: } p t s G \text {-RAT IL5 + JS11 + } \\
\text { OS114 + OS113 / BamHI + MfeI } \\
(p t s G-R 3)\end{array}$ & Schilling et al., 2006 \\
\hline pGP553 & $\begin{array}{l}\mathrm{pAC7} / \text { BamHI }+ \\
\text { EcoRI }\end{array}$ & $\begin{array}{l}\text { CCR: ptsG-RAT IL5 + JS11 + OS112 } \\
\text { / BamHI + MfeI (ptsG-R4) }\end{array}$ & Schilling et al., 2006 \\
\hline
\end{tabular}




\begin{tabular}{|c|c|c|c|}
\hline Plasmid & Construction & Description & Reference \\
\hline pGP554 & $\begin{array}{l}\text { pAC7 / BamHI + } \\
\text { EcoRI }\end{array}$ & $\begin{array}{l}\text { MMR: ptsG-RAT IL5 + JS11 + } \\
\text { OS117 + OS111 + OS118 + OS119 / } \\
\text { BamHI + MfeI }\end{array}$ & \\
\hline pGP555 & $\begin{array}{l}\mathrm{pAC7} / \text { BamHI + } \\
\text { EcoRI }\end{array}$ & $\begin{array}{l}\text { CCR: ptsG-RAT IL5 + JS11 + OS117 } \\
\text { + OS112 / BamHI + MfeI }\end{array}$ & \\
\hline pGP556 & $\begin{array}{l}\mathrm{pAC7} / \text { BamH }+ \\
\text { EcoRI }\end{array}$ & $\begin{array}{l}\text { MMR: ptsG-RAT IL5 + JS11 + } \\
\text { OS111 + OS118 + OS119 / BamHI + } \\
\text { MfeI (ptsG-R6) }\end{array}$ & Schilling et al., 2006 \\
\hline pGP557 & $\begin{array}{l}\mathrm{pAC7} / \text { BamHI + } \\
\text { EcoRI }\end{array}$ & $\begin{array}{l}\text { MMR: ptsG-RAT IL5 + JS11 + } \\
\text { OS110 + OS118 + OS119 / BamHI + } \\
\text { MfeI (ptsG-R5) }\end{array}$ & Schilling et al., 2006 \\
\hline pGP558 & $\begin{array}{l}\mathrm{pAC7} / \text { BamHI + } \\
\text { EcoRI }\end{array}$ & $\begin{array}{l}\text { MMR: ptsG-RAT IL5 + JS11 + } \\
\text { OS } 117+\text { OS } 110+\text { OS } 118+\text { OS } 119 / \\
\text { BamHI + MfeI }\end{array}$ & \\
\hline pGP559 & $\begin{array}{l}\text { pAC7 / BamHI + } \\
\text { EcoRI }\end{array}$ & $\begin{array}{l}\text { CCR: } p t s G \text {-RAT IL5 + JS11 + OS6 + } \\
\text { OS7 + OS8 / BamHI + MfeI }\end{array}$ & \\
\hline pGP560 & $\begin{array}{l}\text { pyk in pBluescript } \\
\text { II SK } \text { SK }^{+} \text {BamHI + } \\
\text { PstI }\end{array}$ & $p y k$-forw $1+p y k$-rev & \\
\hline pGP561 & $\begin{array}{l}\mathrm{pAC7} / \text { BamHI + } \\
\text { EcoRI }\end{array}$ & $\begin{array}{l}\text { MMR: ptsG-RAT IL5 + JS11 + OS30 } \\
+ \text { OS31 + OS32 / BamHI + MfeI } \\
(p t s G-R 8)\end{array}$ & Schilling et al., 2006 \\
\hline pGP562 & $\begin{array}{l}\text { pBgaB /BamHI + } \\
\text { EcoRI }\end{array}$ & $\begin{array}{l}\text { PCR: ptsG-RAT IL5 + JS11 / BamHI } \\
\text { + MfeI }\end{array}$ & \\
\hline pGP563 & $\begin{array}{l}\text { pWH844 / BamHI } \\
+ \text { PstI }\end{array}$ & eno forw $1+$ eno rev & \\
\hline pGP564 & $\begin{array}{l}\mathrm{pAC7} / \text { BamH }+ \\
\text { EcoRI }\end{array}$ & $\begin{array}{l}\text { PCR: } s a c B \text { OS49 + OS50 / BamHI + } \\
\text { EcoRI }\end{array}$ & Schilling et al., 2006 \\
\hline pGP565 & $\begin{array}{l}\text { pAC7 / BamHI + } \\
\text { EcoRI }\end{array}$ & $\begin{array}{l}\text { CCR: sacB OS } 49+\text { OS50 + OS55 / } \\
\text { BamHI + EcoRI (sacB-R10) }\end{array}$ & Schilling et al., 2006 \\
\hline pGP566 & $\begin{array}{l}\mathrm{pAC7} / \text { BamHI + } \\
\text { EcoRI }\end{array}$ & $\begin{array}{l}\text { CCR: sacB OS49 + OS50 + OS47 / } \\
\text { BamHI + EcoRI (sacB-R11) }\end{array}$ & Schilling et al., 2006 \\
\hline pGP567 & $\begin{array}{l}\mathrm{pAC7} / \text { BamH }+ \\
\text { EcoRI }\end{array}$ & $\begin{array}{l}\text { MMR: } s a c B \text { OS } 49+\text { OS } 50+\text { OS55 + } \\
\text { OS48 + OS54 / BamHI + EcoRI } \\
(s a c B-R 12)\end{array}$ & \\
\hline pGP568 & $\begin{array}{l}\mathrm{pAC7} / \text { BamH }+ \\
\text { EcoRI }\end{array}$ & $\begin{array}{l}\text { MMR: } s a c B \text { OS } 49+\text { OS } 50+\text { OS } 47+ \\
\text { OS48 + OS54 / BamHI + EcoRI } \\
(s a c B-R 13)\end{array}$ & Schilling et al., 2006 \\
\hline pGP569 & $\begin{array}{l}\mathrm{pAC7} / \text { BamHI + } \\
\text { EcoRI }\end{array}$ & $\begin{array}{l}\text { CCR: sacB OS49 + OS50 + OS61 / } \\
\text { BamHI + EcoRI (sacB-R2) }\end{array}$ & Schilling et al., 2006 \\
\hline pGP570 & $\begin{array}{l}\text { pWH844 / BamHI } \\
+ \text { PstI }\end{array}$ & $\begin{array}{l}\text { OS } 78+\text { OS79; introduction of } \\
\text { thrombin restriction site; insert is } \\
\text { obtained by oligonucleotide annealing }\end{array}$ & \\
\hline pGP571 & $\begin{array}{l}\text { pGP570 / SalI + } \\
\text { HindIII }\end{array}$ & $\begin{array}{l}\text { SacT-RBD; N-terminal fusion to } \\
\text { His }_{6} \text {-tag; can be cleaved off by } \\
\text { Thrombin }\end{array}$ & \\
\hline
\end{tabular}




\begin{tabular}{|c|c|c|c|}
\hline Plasmid & Construction & Description & Reference \\
\hline pGP572 & $\begin{array}{l}\text { pGP570 / SalI + } \\
\text { HindIII }\end{array}$ & $\begin{array}{l}\text { LicT-RBD; N-terminal fusion to } \\
\text { His }_{6} \text {-tag; can be cleaved off by } \\
\text { Thrombin }\end{array}$ & \\
\hline pGP573 & $\begin{array}{l}\text { pGP570 / SalI + } \\
\text { HindIII }\end{array}$ & $\begin{array}{l}\text { SacY-RBD; N-terminal fusion to } \\
\text { His }_{6} \text {-tag; can be cleaved off by } \\
\text { Thrombin }\end{array}$ & \\
\hline pGP574 & $\begin{array}{l}\mathrm{pET} 3 \mathrm{C} / \mathrm{NdeI}+ \\
\text { BamHI }\end{array}$ & $\begin{array}{l}\text { OS91+OS92; cloning of C-terminal } \\
\text { Strep-tag into pET3C; introduction of } \\
\text { SacI and displacing of } B a m H I \\
\text { restriction site; insert is obtained by } \\
\text { oligonucleotide annealing }\end{array}$ & Schilling et al., 2006 \\
\hline pGP575 & $\begin{array}{l}\text { pGP574 / NdeI + } \\
\text { BamHI }\end{array}$ & $\begin{array}{l}\text { GlcT-RBD; C-terminal fusion to } \\
\text { Strep-tag with OS93 + OS94 }\end{array}$ & Schilling et al., 2006 \\
\hline pGP576 & $\begin{array}{l}\text { pGP574 / NdeI + } \\
\text { BamHI }\end{array}$ & $\begin{array}{l}\text { LicT-RBD C-terminal fusion to } \\
\text { Strep-tag with OS95 + OS96 }\end{array}$ & Schilling et al., 2006 \\
\hline pGP577 & $\begin{array}{l}\text { pGP574 / NdeI }+ \\
\text { BamHI }\end{array}$ & $\begin{array}{l}\text { SacT-RBD C-terminal fusion to } \\
\text { Strep-tag with OS97 + OS98 }\end{array}$ & Schilling et al., 2006 \\
\hline pGP578 & $\begin{array}{l}\text { pGP574 / NdeI + } \\
\text { BamHI }\end{array}$ & $\begin{array}{l}\text { SacY-RBD C-terminal fusion to } \\
\text { Strep-tag with OS } 99+\text { OS } 100\end{array}$ & \\
\hline pGP579 & $\begin{array}{l}\text { pBluescript / } \\
\text { BamHI + ApaI }\end{array}$ & PCR OS56 + OS57 ( $p d h B$ knock out) & \\
\hline pGP580 & $\begin{array}{l}\mathrm{pAC7} / \text { BamHI + } \\
\text { EcoRI }\end{array}$ & $\begin{array}{l}\text { CCR: } s a c B \text { OS } 49+\text { OS50 + OS62 / } \\
\text { BamHI + EcoRI (sacB-R1) }\end{array}$ & Schilling et al., 2006 \\
\hline pGP581 & $\begin{array}{l}\mathrm{pAC7} / \text { BamHI + } \\
\text { EcoRI }\end{array}$ & $\begin{array}{l}\text { MMR: } s a c B \text { OS } 49+\text { OS } 50+\text { OS71 + } \\
\text { OS72 / BamHI + EcoRI }\end{array}$ & \\
\hline pGP582 & $\begin{array}{l}\mathrm{pAC7} / \text { BamH }+ \\
\text { EcoRI }\end{array}$ & $\begin{array}{l}\text { MMR: } \operatorname{sacB} \text { OS } 49+\text { OS50 + OS61 + } \\
\text { OS71 + OS72 / BamHI + EcoRI } \\
(\operatorname{sacB}-\mathrm{R} 7)\end{array}$ & Schilling et al., 2006 \\
\hline pGP583 & $\begin{array}{l}\text { pAC7 / BamHI + } \\
\text { EcoRI }\end{array}$ & $\begin{array}{l}\text { MMR: } s a c B \text { OS } 49+\text { OS } 50+\text { OS62 + } \\
\text { OS71 + OS72 / BamHI + EcoRI } \\
(s a c B-R 9)\end{array}$ & Schilling et al., 2006 \\
\hline pGP584 & $\begin{array}{l}\mathrm{pAC7} / \text { BamHI }+ \\
\text { EcoRI }\end{array}$ & $\begin{array}{l}\text { CCR: } s a c B \text { OS } 49+\text { OS50 + OS77 / } \\
\text { BamHI + EcoRI }\end{array}$ & \\
\hline pGP585 & $\begin{array}{l}\text { pAC7 / BamHI + } \\
\text { EcoRI }\end{array}$ & $\begin{array}{l}\text { MMR: } s a c B \text { OS } 49+\text { OS50 + OS62 + } \\
\text { OS77 / BamHI + EcoRI }\end{array}$ & \\
\hline pGP586 & $\begin{array}{l}\mathrm{pAC} 7 / \text { BamH }+ \\
\text { EcoRI }\end{array}$ & $\begin{array}{l}\text { MMR: } s a c B \text { OS } 49+\text { OS } 50+\text { OS76 + } \\
\text { OS72 / BamHI + EcoRI }\end{array}$ & \\
\hline pGP587 & $\begin{array}{l}\mathrm{pAC7} / \text { BamH }+ \\
\text { EcoRI }\end{array}$ & $\begin{array}{l}\text { MMR: sacB OS49 + OS50 + OS61 + } \\
\text { OS76 + OS72 (sacB-R8) / BamHI + } \\
\text { EcoRI }\end{array}$ & Schilling et al., 2006 \\
\hline pGP588 & $\begin{array}{l}\mathrm{pAC7} / \text { BamHI }+ \\
\text { EcoRI }\end{array}$ & $\begin{array}{l}\text { MMR: } s a c B \text { OS } 49+\text { OS } 50+\text { OS } 62+ \\
\text { OS76 + OS72 }\end{array}$ & \\
\hline pGP589 & $\begin{array}{l}\text { pAC7 / BamHI + } \\
\text { EcoRI }\end{array}$ & $\begin{array}{l}\text { MMR: sacB OS } 49+\text { OS } 50+\text { OS } 88 \\
(s a c B-R 3) / \text { BamHI }+E c o R I\end{array}$ & Schilling et al., 2006 \\
\hline
\end{tabular}




\begin{tabular}{|c|c|c|c|}
\hline Plasmid & Construction & Description & Reference \\
\hline pGP590 & $\begin{array}{l}\mathrm{pAC7} / \text { BamHI }+ \\
\text { EcoRI }\end{array}$ & $\begin{array}{l}\text { MMR: } s a c B \text { OS } 49+\text { OS50 + OS } 89 \\
(S a c B-R 5) / B a m H I+E c o R I\end{array}$ & Schilling et al., 2006 \\
\hline pGP591 & $\begin{array}{l}\mathrm{pAC7} / \text { BamHI + } \\
\text { EcoRI }\end{array}$ & $\begin{array}{l}\text { MMR: ptsG OS66 + OS118 + OS119 } \\
\text { / BamHI + MfeI }(p t s G-\mathrm{R} 7)\end{array}$ & Schilling et al., 2006 \\
\hline pGP592 & $\begin{array}{l}\mathrm{pAC7} / \text { BamHI + } \\
\text { EcoRI }\end{array}$ & $\begin{array}{l}\text { MMR: ptsG IL5 + JS11 + OS90 + } \\
\text { OS119; pGP557 / BamHI + MfeI }\end{array}$ & \\
\hline pGP593 & $\begin{array}{l}\text { pGP574 / NdeI + } \\
\text { BamHI }\end{array}$ & $\begin{array}{l}\text { SacT; C-terminal fusion to Strep-tag } \\
\text { with OS97 + OS108 }\end{array}$ & \\
\hline pGP594 & $\begin{array}{l}\text { pGP574 / NdeI + } \\
\text { BamHI }\end{array}$ & $\begin{array}{l}\text { SacY; C-terminal fusion to Strep-tag } \\
\text { with OS99 + OS109 }\end{array}$ & \\
\hline pGP595 & $\begin{array}{l}\text { pAC7 / BamHI + } \\
\text { EcoRI }\end{array}$ & $\begin{array}{l}\text { CCR: } s a c B \text { OS } 49+\text { OS50 + OS102 / } \\
\text { BamHI + EcoRI (sacB-R6) }\end{array}$ & Schilling et al., 2006 \\
\hline pGP596 & $\begin{array}{l}\mathrm{pAC7} / \text { BamHI + } \\
\text { EcoRI }\end{array}$ & $\begin{array}{l}\text { CCR: sacB OS } 49+\text { OS } 50+\text { OS } 105 \text { / } \\
\text { BamHI + EcoRI (sacB-R4) }\end{array}$ & Schilling et al., 2006 \\
\hline pGP597 & $\begin{array}{l}\mathrm{pAC7} / \text { BamHI + } \\
\text { EcoRI }\end{array}$ & $\begin{array}{l}\text { MMR: sacB OS } 49+\text { OS } 50+\text { OS } 61+ \\
\text { OS77 / BamHI + EcoRI }\end{array}$ & Schilling et al., 2006 \\
\hline pGP598 & $\begin{array}{l}\mathrm{pAC7} / \text { BamHI + } \\
\text { EcoRI }\end{array}$ & $\begin{array}{l}\text { CCR: pGP592 + IL5 + JS11 + } \\
\text { OS103 / BamHI + MfeI }\end{array}$ & \\
\hline pGP599 & $\begin{array}{l}\mathrm{pAC7} / \text { BamHI + } \\
\text { EcoRI }\end{array}$ & $\begin{array}{l}\text { CCR: pGP592 + IL5 + JS11 + } \\
\text { OS104 / BamHI + MfeI }\end{array}$ & \\
\hline pGP1100 & $\begin{array}{l}\text { pWH844 / BamHI } \\
+ \text { PstI }\end{array}$ & $p y k$ forw $1+p y k$ rev & \\
\hline pGP1101 & $\begin{array}{l}\text { pWH844 / BamHI } \\
+ \text { PstI }\end{array}$ & $p g m$ forw $1+p g m$ rev & \\
\hline pGP1102 & $\begin{array}{l}\text { pWH844 / SalI + } \\
\text { HindIII }\end{array}$ & $p g k$ forw $1+p g k$ rev & \\
\hline
\end{tabular}

\section{Strains}

Table 17. Strains used in this work

\begin{tabular}{|c|c|c|}
\hline Strain & Genotype & Reference \\
\hline \multicolumn{3}{|c|}{ Escherichia coli } \\
\hline DH5 $\alpha$ & 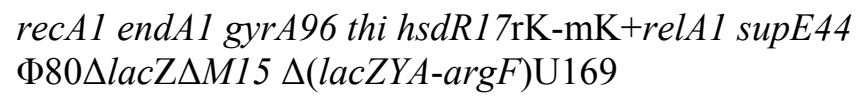 & Sambrook et al., 1989 \\
\hline BL21 (DE3) & $\begin{array}{l}\text { F- lon ompT rBmB hsdS gal (cIts857ind1 Sam7 nin5 } \\
\text { lacUV5- T7 gene1) }\end{array}$ & Sambrook et al., 1989 \\
\hline \multicolumn{3}{|c|}{ Bacillus subtilis } \\
\hline 168 & $\operatorname{trp} C 2$ & Laboratory collection \\
\hline BGW10 & $\operatorname{trpC} 2$ lys-3 $\Delta$ licTS ::erm & Krüger and Hecker, 1995 \\
\hline
\end{tabular}




\begin{tabular}{|c|c|c|}
\hline Strain & Genotype & Reference \\
\hline BSIP1114 & $\operatorname{trpC} 2$ amyE::(pta-lacZ cat $)$ & $\begin{array}{l}\text { Presecan-Siedel et al., } \\
1999\end{array}$ \\
\hline GM1010 & $\operatorname{sac} X Y \Delta 3 \operatorname{sac} B \Delta 23 \operatorname{sacT} \Delta 4$ & Le Coq et al., 1995 \\
\hline GM1112 & $\begin{array}{l}\text { sacXY } 3 \text { sacB } 23 \text { sacT } 4 \text { bglP::Tn } 10 \text { erm amyE::(sacB- } \\
\text { lacZ phl) }\end{array}$ & Le Coq et al., 1995 \\
\hline GP109 & 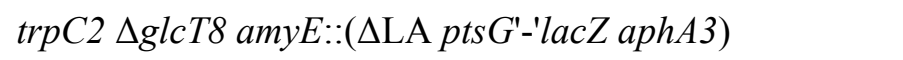 & Bachem and Stülke, 1998 \\
\hline GP133 & $\operatorname{trpC2}$ amyE::(fbaA-lacZ cat) & Ludwig et al., 2001 \\
\hline GP150 & $\operatorname{trpC2} \triangle g \mathrm{glcT}$ amyE::('lacZ cat) & Langbein et al., 1999 \\
\hline GP151 & $\operatorname{trp} C 2 \operatorname{amyE}::\left(\Delta \mathrm{LA}_{\mathrm{C} 75 \mathrm{G} \mathrm{C} 76 \mathrm{G}} p t s G^{\prime}-{ }^{\prime} l a c Z\right.$ aphA3) & Langbein et al., 1999) \\
\hline GP169 & 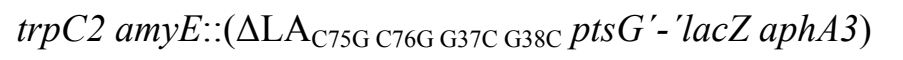 & Schilling et al., 2004 \\
\hline GP172 & $\operatorname{trp} C 2 \operatorname{amyE}::\left(\Delta \mathrm{CA}_{\mathrm{G} 11 \mathrm{~A} \mathrm{G} 26 \mathrm{~A}} p t s G^{\prime}-{ }^{\prime} a p h A 3-' l a c Z c a t\right)$ & Schilling et al., 2004 \\
\hline GP173 & $\operatorname{trp} C 2 \operatorname{amyE}::\left(\Delta \mathrm{CA}_{\mathrm{G} 11 \mathrm{C} \mathrm{G} 26 \mathrm{~A}} p t s G^{\prime}-{ }^{\prime} a p h A 3-' l a c Z c a t\right)$ & Schilling et al., 2004 \\
\hline GP174 & $\operatorname{trp} C 2$ amyE::( $\triangle \mathrm{CA}$ ptsG'-'aphA3-'lacZ cat) & Schilling et al., 2004 \\
\hline GP175 & $\operatorname{trp} C 2$ amyE::( $\triangle \mathrm{LA}_{\mathrm{U}} \mathrm{c}$ ptsG'-'lacZ aphA3) & Schilling et al., 2004 \\
\hline GP176 & $\operatorname{trp} C 2 \operatorname{amyE::(\Delta \mathrm {LA}_{\mathrm {A}8\mathrm {G}}ptsG^{\prime }-{}^{\prime }lacZ\text {aphA3})}$ & Schilling et al., 2004 \\
\hline GP177 & $\operatorname{trp} C 2 \operatorname{amyE::(\Delta \mathrm {LA}_{\mathrm {U}}\mathrm {C}\text {A8G}} p t s G^{\prime}-{ }^{\prime} l a c Z$ aphA3) & Schilling et al., 2004 \\
\hline GP179 & 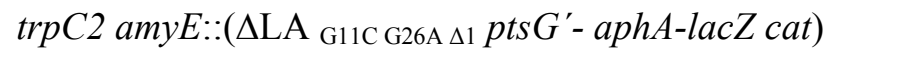 & Schilling et al., 2004 \\
\hline GP181 & $\operatorname{trp} C 2 \operatorname{amyE}::\left(\Delta \mathrm{LA}_{\mathrm{G} 11 \mathrm{C} \mathrm{G} 26 \mathrm{~A} \mathrm{G} 38 \mathrm{~A}} p t s G^{\prime}-a p h A 3-l a c Z c a t\right)$ & Schilling et al., 2004 \\
\hline GP182 & $\operatorname{trpC} 2 \operatorname{amyE}::\left(\Delta \mathrm{LA}_{\mathrm{G} 11 \mathrm{C} \mathrm{G} 26 \mathrm{~A} \text { G47U }} p t s G^{\prime}-a p h A 3-l a c Z c a t\right)$ & Schilling et al., 2004 \\
\hline GP183 & 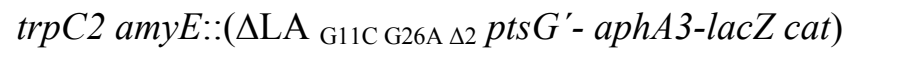 & Schilling et al., 2004 \\
\hline GP184 & $\operatorname{trp} C 2$ amyE: $:\left(\Delta \mathrm{LA}_{\mathrm{G} 11 \mathrm{C} \text { G26A C75U }} p t s G^{\prime}-\right.$ aphA3-lacZ cat $)$ & Schilling et al., 2004 \\
\hline GP185 & $\operatorname{trp} C 2 a m y E::\left(\Delta \mathrm{LA}_{\mathrm{G} 11 \mathrm{C} \mathrm{G} 26 \mathrm{~A} \mathrm{G} 90 \mathrm{~A}} p t s G^{\prime}-a p h A 3-l a c Z c a t\right)$ & Schilling et al., 2004 \\
\hline GP186 & 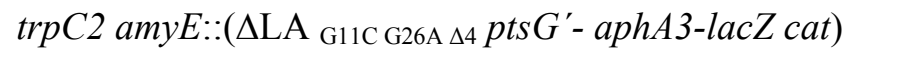 & Schilling et al., 2004 \\
\hline GP187 & $\operatorname{trp} C 2 \operatorname{amyE}::\left(\Delta \mathrm{LA}_{\mathrm{G} 11 \mathrm{C} \mathrm{G} 26 \mathrm{~A} \mathrm{G} 72 \mathrm{U}} p t s G^{\prime}-a p h A 3-l a c Z c a t\right)$ & Schilling et al., 2004 \\
\hline GP188 & 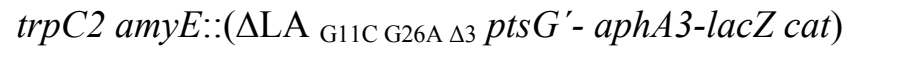 & Schilling et al., 2004 \\
\hline GP189 & $\operatorname{trp} C 2 a m y E::\left(\Delta \mathrm{LA}_{\mathrm{G} 11 \mathrm{C} \mathrm{G} 26 \mathrm{~A} \text { G28U }} p t s G^{\prime}-a p h A 3-l a c Z c a t\right)$ & Schilling et al., 2004 \\
\hline GP190 & $\operatorname{trp} C 2 \operatorname{amyE}::\left(\Delta \mathrm{LA}_{\mathrm{G} 11 \mathrm{C} \mathrm{G} 26 \mathrm{~A} \mathrm{C} 75 \mathrm{~A}} p t s G^{\prime}-a p h A 3-l a c Z c a t\right)$ & Schilling et al., 2004 \\
\hline GP191 & $\operatorname{trp} C 2 \operatorname{amyE}::\left(\Delta \mathrm{LA}_{\mathrm{G} 11 \mathrm{C} \mathrm{G} 26 \mathrm{~A} \text { G47U }} p t s G^{\prime}-a p h A 3-l a c Z c a t\right)$ & Schilling et al., 2004 \\
\hline GP205 & $\operatorname{trp} C 2$ amyE::(citB-lacZ cat $)$ & Blencke et al., 2006 \\
\hline GP216 & $\operatorname{trpC2}$ amyE::(pdhA-lacZ cat $)$ & Schilling et al., 2007 \\
\hline GP250 & $\operatorname{trpC2}$ amyE::(nrgA-lacZ aphA3) & Detsch and Stülke, 2003 \\
\hline GP268 & $\operatorname{trpC} 2$ amyE::(glnR-lacZ aphA3) & Schilling et al., 2007 \\
\hline GP313 & $\operatorname{trpC2}$ amyE::(cggR-lacZ cat) & Ludwig et al., 2001 \\
\hline GP314 & $\operatorname{trpC} 2$ amyE::(pgi-lacZ cat $)$ & Ludwig et al., 2001 \\
\hline GP315 & $\operatorname{trpC} 2$ amyE::(pfkA-lacZ cat) & Ludwig et al., 2001 \\
\hline GP317 & $\operatorname{trpC2}$ amyE::(pgk-lacZ cat $)$ & Ludwig et al., 2001 \\
\hline GP342 & $\operatorname{trp} C 2$ amyE::(gltA-lacZ aphA3) & Wacker et al., 2003 \\
\hline
\end{tabular}




\begin{tabular}{|c|c|c|}
\hline Strain & Genotype & Reference \\
\hline QB5435 & $\operatorname{trp} C 2 \Delta p t s G:: c a t$ & Stülke et al., 1997 \\
\hline QB5448 & $\operatorname{trp} C 2$ amyE::( $\triangle L A$ pts $G^{\prime}-' l a c Z$ aphA3) & Stülke et al., 1997 \\
\hline QB5449 & $\operatorname{trp} C 2$ pts $G:: c a t$ amyE::( $\Delta L A$ pts $G-l a c Z$ aphA3) & Stülke et al., 1997 \\
\hline QB5556 & $\operatorname{trpC} 2$ amyE::(rocD'-'lacZ cat) & Gardan et al., 1995 \\
\hline QB7041 & 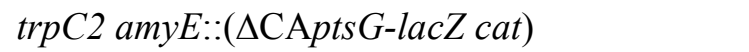 & Stülke et al., 1997 \\
\hline
\end{tabular}

Table 18. B. subtilis strains constructed in this work

\begin{tabular}{|c|c|c|c|}
\hline Name & Genotype & Construction $^{\mathrm{a}}$ & Reference \\
\hline GP366 & 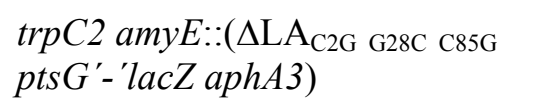 & pGP532 $\rightarrow$ GP368 & Schilling et al., 2004 \\
\hline GP367 & $\begin{array}{l}\operatorname{trp} C 2 \text { amyE }:\left(\Delta \mathrm{LA}_{\mathrm{C} 2 \mathrm{G}} \text { pts } G^{\prime}-\right. \\
\text { 'lacZ aph } 3)\end{array}$ & pGP533 $\rightarrow 168$ & Schilling et al., 2004 \\
\hline GP368 & $\begin{array}{l}\operatorname{trp} C 2 \text { amyE::( }\left(\Delta \mathrm{LA}_{\mathrm{G} 28 \mathrm{C}} \mathrm{C} 85 \mathrm{G}\right. \\
\text { pts } G^{\prime}-{ }^{\prime} \text { lac } Z \text { aphA3) }\end{array}$ & pGP534 $\rightarrow 168$ & Schilling et al., 2004 \\
\hline GP369 & 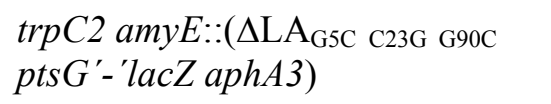 & pGP535 $\rightarrow 168$ & Schilling et al., 2004 \\
\hline GP370 & 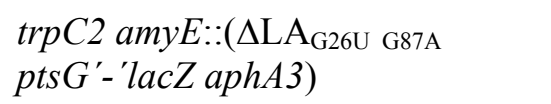 & pGP536 $\rightarrow 168$ & Schilling et al., 2004 \\
\hline GP371 & $\begin{array}{l}\operatorname{trp} C 2 \text { amyE }::\left(\Delta \mathrm{LA}_{\mathrm{U} 7 \mathrm{G}} p t s G^{\prime}-\right. \\
\text { 'lacZ aphA3) }\end{array}$ & pGP537 $\rightarrow 168$ & Schilling et al., 2004 \\
\hline GP375 & $\begin{array}{l}\operatorname{trp} C 2 \text { amyE }::\left(\Delta \mathrm{LA}_{\mathrm{G} 3 \mathrm{C}} p t s G^{\prime}-\right. \\
\text { 'lacZ aph } 3)\end{array}$ & pGP541 $\rightarrow 168$ & Schilling et al., 2004 \\
\hline GP376 & $\begin{array}{l}\operatorname{trp} C 2 \text { amyE::( } \triangle \mathrm{LA} \\
\left.\left(\mathrm{RAT}^{* 3}\right) p t s G^{\prime}-{ }^{\prime} l a c Z \text { aph } A 3\right)\end{array}$ & pGP542 $\rightarrow 168$ & Schilling et al., 2004 \\
\hline GP377 & $\begin{array}{l}\operatorname{trp} C 2 \text { amyE }::\left(\Delta \mathrm{LA}_{\mathrm{U} 6 \mathrm{G}} p t s G^{\prime}-\right. \\
\text { 'lacZ aph } 3)\end{array}$ & pGP543 $\rightarrow 168$ & Schilling et al., 2004 \\
\hline GP378 & $\begin{array}{l}\operatorname{trpC} 2 \text { amyE }::\left(\Delta \mathrm{LA}_{\mathrm{U} 6 \mathrm{~A}} p t s G^{\prime}-\right. \\
\text { 'lacZ aphA3) }\end{array}$ & pGP544 $\rightarrow 168$ & Schilling et al., 2004 \\
\hline GP379 & $\begin{array}{l}\operatorname{trpC} 2 \text { amyE }::\left(\Delta \mathrm{LA}_{\mathrm{U} 25 \mathrm{G}} \mathrm{A} 88 \mathrm{C}\right. \\
\text { pts } G^{\prime}-{ }^{\prime} l a c Z \text { aphA3) }\end{array}$ & pGP545 $\rightarrow 168$ & Schilling et al., 2004 \\
\hline GP380 & $\begin{array}{l}\operatorname{trp} C 2 \text { amyE }::\left(\Delta \mathrm{LA}_{\mathrm{A} 27 \mathrm{U}} \mathrm{U} 86 \mathrm{~A}\right. \\
p t s G^{\prime}-' \text { lacZ } \text { aphA3) }\end{array}$ & pGP546 $\rightarrow 168$ & Schilling et al., 2004 \\
\hline GP381 & $\begin{array}{l}\operatorname{trp} C 2 \text { amyE }::\left(\Delta \mathrm{LA}_{\mathrm{A} 27 \mathrm{G}} \mathrm{U} 86 \mathrm{C}\right. \\
\text { pts } G^{\prime}-' \text { lac } Z \text { aphA3) }\end{array}$ & pGP547 $\rightarrow 168$ & Schilling et al., 2004 \\
\hline GP385 & $\begin{array}{l}\operatorname{trp} C 2 \text { amyE::( } \triangle \mathrm{LA}_{\mathrm{R} 1} p t s G^{\prime}-{ }^{\prime} l a c Z \\
\text { aphA3) }\end{array}$ & pGP549 $\rightarrow 168$ & Schilling et al., 2004 \\
\hline GP386 & $\begin{array}{l}\operatorname{trpC} 2 \text { amyE::(}\left(\Delta \mathrm{LA}_{\mathrm{R} 1} p t s G^{\prime}-\right. \\
\text { 'lacZ aphA3) } \Delta g l c T 8\end{array}$ & pGP549 $\rightarrow$ GP150 & Schilling et al., 2004 \\
\hline GP387 & 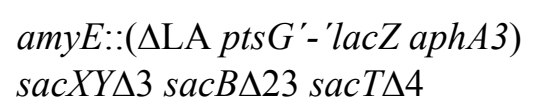 & pGP66 $\rightarrow$ GM1010 & Schilling et al., 2004 \\
\hline
\end{tabular}




\begin{tabular}{|c|c|c|c|}
\hline Name & Genotype & Construction $^{\text {a }}$ & Reference \\
\hline GP388 & $\begin{array}{l}\text { amyE::( } \Delta \mathrm{LA}_{\mathrm{R} 1} p t s G^{\prime}-{ }^{\prime} l a c Z \\
\text { aphA3) sacXY } 3 \text { sacB } \Delta 23 \\
\text { sacT } 4\end{array}$ & pGP549 $\rightarrow$ GM1010 & Schilling et al., 2004 \\
\hline GP389 & 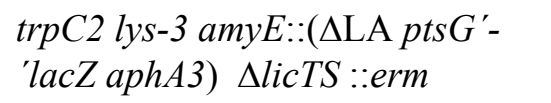 & pGP66 $\rightarrow$ BGW10 & Schilling et al., 2004 \\
\hline GP390 & 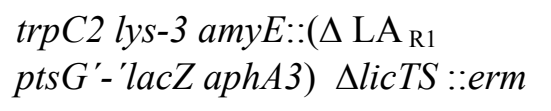 & pGP549 $\rightarrow$ BGW10 & Schilling et al., 2004 \\
\hline GP391 & prototrophic & $\begin{array}{l}\text { GM1010 chrom. } \rightarrow \\
168\end{array}$ & \\
\hline GP392 & $\begin{array}{l}\operatorname{trp} C 2 \text { amyE }::\left(\Delta \mathrm{LA}_{\mathrm{G} 26 \mathrm{~T}}^{\mathrm{G} 87 \mathrm{~A}}\right. \\
\text { pts } G^{\prime}-' \text { lacZ aphA3) } \Delta g l c T 8\end{array}$ & pGP536 $\rightarrow$ GP150 & Schilling et al., 2004 \\
\hline GP393 & $\begin{array}{l}\operatorname{trp} C 2 \text { amyE::( } \Delta \mathrm{LA}_{\mathrm{R} 2} p t s G^{\prime}-\operatorname{l}^{\prime} l a c Z \\
\text { aphA3) } \Delta g l c T 8\end{array}$ & pGP550 $\rightarrow$ GP150 & \\
\hline GP394 & $\begin{array}{l}\operatorname{trp} C 2 \text { amyE::(}\left(\Delta \mathrm{LA}_{\mathrm{A} 4-\mathrm{ins} \mathrm{U} 18 \mathrm{G}}\right. \\
p t s G^{\prime}-{ }^{\prime} l a c Z \text { aphA3) } \Delta g l c T 8\end{array}$ & pGP551 $\rightarrow$ GP150 & \\
\hline GP395 & $\begin{array}{l}\operatorname{trp} C 2 \text { amyE::( } \Delta \mathrm{LA}_{\mathrm{R} 3} p t s G^{\prime}-{ }^{\prime} l a c Z \\
\text { aphA3) } \Delta g l c T 8\end{array}$ & pGP552 $\rightarrow$ GP150 & \\
\hline GP396 & $\begin{array}{l}\operatorname{trp} C 2 \text { amyE::( } \Delta \mathrm{LA}_{\mathrm{R} 4} p t s G^{\prime}-' l a c Z \\
\text { aphA3) } \Delta g l c T 8\end{array}$ & pGP553 $\rightarrow$ GP150 & Schilling et al., 2006 \\
\hline GP397 & $\begin{array}{l}\operatorname{trpC} 2 \text { amyE::( }\left(\Delta \mathrm{LA}_{\mathrm{A} 4-\mathrm{ins}} \mathrm{A} 4 \mathrm{U} \mathrm{U} 25 \mathrm{~A}\right. \\
\text { A88U G3A U18G } p t s G^{\prime}-{ }^{\prime} l a c Z \text { aphA3) } \\
\Delta g l c T 8\end{array}$ & pGP554 $\rightarrow$ GP150 & \\
\hline GP398 & $\begin{array}{l}\operatorname{trp} C 2 \text { amyE::( }\left(\Delta \mathrm{LA}_{\mathrm{A} 4-\mathrm{ins} \mathrm{G} 3 \mathrm{~A} \mathrm{U} 18 \mathrm{G}}\right. \\
p t s G^{\prime}-' \operatorname{lac} Z \text { aphA3) } \Delta g l c T 8\end{array}$ & pGP555 $\rightarrow$ GP150 & \\
\hline GP399 & $\begin{array}{l}\operatorname{trpC2} \text { amyE::( }\left(\Delta \mathrm{LA}_{\mathrm{R} 6} p t s G^{\prime}-{ }^{\prime} l a c Z\right. \\
\text { aphA3) } \Delta g l c T 8\end{array}$ & pGP556 $\rightarrow$ GP150 & Schilling et al., 2006 \\
\hline GP400 & $\begin{array}{l}\operatorname{trp} C 2 \text { amyE }::\left(\Delta \mathrm{LA}_{\mathrm{R} 5} p t s G^{\prime}-{ }^{\prime} l a c Z\right. \\
\text { aphA3) } \Delta g l c T 8\end{array}$ & pGP557 $\rightarrow$ GP150 & Schilling et al., 2006 \\
\hline GP401 & $\begin{array}{l}\operatorname{trpC} 2 \text { amyE::( }\left(\mathrm{LA}_{\mathrm{A} 4-\mathrm{ins}} \mathrm{A} 4 \mathrm{U} \mathrm{U} 25 \mathrm{~A}\right. \\
\left.\text { A88U U18G } p t s G^{\prime}-{ }^{\prime} l a c Z \text { aphA3 }\right) \\
\Delta g l c T 8\end{array}$ & pGP558 $\rightarrow$ GP150 & \\
\hline GP402 & $\begin{array}{l}\operatorname{trpC} 2 \text { amyE::( } \Delta \mathrm{LA}_{\mathrm{R} 5} \text { ptsG'- } \\
\text { 'lacZ aphA3) } \Delta \text { licTS ::erm }\end{array}$ & pGP557 $\rightarrow$ GP427 & Schilling et al., 2006 \\
\hline GP403 & 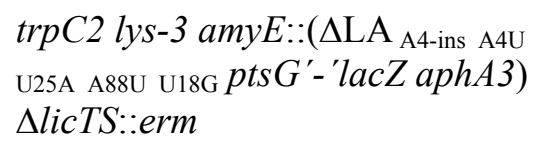 & pGP558 $\rightarrow$ GP427 & \\
\hline GP404 & $\begin{array}{l}\operatorname{trp} C 2 \text { amyE::( }\left(\Delta \mathrm{LA}_{\mathrm{R} 5} p t s G^{\prime}-{ }^{\prime} l a c Z\right. \\
\text { aphA3) }\end{array}$ & pGP557 $\rightarrow 168$ & Schilling et al., 2006 \\
\hline GP405 & $\begin{array}{l}\operatorname{trp} C 2 \text { amyE: }:\left(\Delta \mathrm{LA}_{\mathrm{A} 4-\mathrm{ins}} \mathrm{A} 4 \mathrm{U} \mathrm{U} 25 \mathrm{~A}\right. \\
\mathrm{A} 88 \mathrm{U} \mathrm{U} 18 \mathrm{G} \operatorname{pts} G^{\prime}-{ }^{\prime} l a c Z \text { aphA3) }\end{array}$ & pGP558 $\rightarrow 168$ & \\
\hline GP406 & $\begin{array}{l}\operatorname{trp} C 2 \text { amyE::( } \triangle \mathrm{LA}_{\mathrm{U} 7 \mathrm{G}} \mathrm{G} 26 \mathrm{U} \mathrm{C} 87 \mathrm{~A} \\
\text { pts } G^{\prime}-{ }^{\prime} l a c Z \text { aphA3) }\end{array}$ & pGP559 $\rightarrow 168$ & \\
\hline
\end{tabular}




\begin{tabular}{|c|c|c|c|}
\hline Name & Genotype & Construction $^{\text {a }}$ & Reference \\
\hline GP407 & $\begin{array}{l}\operatorname{trp} C 2 \text { lys-3 amyE::( }\left(\Delta \mathrm{LA}_{\mathrm{A} 4-\mathrm{ins} \mathrm{G} 3 \mathrm{~A}}\right. \\
\mathrm{U} 18 \mathrm{G} \text { pts } G^{\prime}-{ }^{\prime} \text { lacZ aphA3) } \\
\Delta \text { licTS }:: \mathrm{erm}\end{array}$ & pGP555 $\rightarrow$ GP427 & \\
\hline GP408 & $\begin{array}{l}\operatorname{trpC2} \text { amyE::(}\left(\Delta \mathrm{LA}_{\mathrm{R} 6} p t s G^{\prime}-{ }^{\prime} l a c Z\right. \\
\text { aphA3) }\end{array}$ & pGP556 $\rightarrow 168$ & Schilling et al., 2006 \\
\hline GP409 & $\begin{array}{l}\operatorname{trp} C 2 \text { amyE::(}\left(\Delta \mathrm{LA}_{\mathrm{R} 6} p t s G^{\prime}-l^{\prime} l a c Z\right. \\
\text { aphA3) } \Delta \text { licTS::erm }\end{array}$ & pGP556 $\rightarrow$ GP427 & Schilling et al., 2006 \\
\hline GP410 & $\begin{array}{l}\operatorname{trp} C 2 \text { amyE::( }\left(\Delta \mathrm{LA}_{\mathrm{A} 4-\mathrm{ins}} \mathrm{A} 4 \mathrm{U} \mathrm{U} 25 \mathrm{~A}\right. \\
\text { A88U G3A U18G } p t s G^{\prime}-' l a c Z \text { aphA3) }\end{array}$ & pGP554 $\rightarrow 168$ & \\
\hline GP411 & $\begin{array}{l}\operatorname{trpC} 2 \text { amyE::( }\left(\mathrm{LA}_{\mathrm{A} 4-\text { ins } \mathrm{A} 4 \mathrm{U}} \mathrm{U} 25 \mathrm{~A}\right. \\
\text { A88U G3A U18G } p t s G^{\prime}-{ }^{\prime} \text { lacZ aphA3) } \\
\Delta \text { licTS ::erm }\end{array}$ & pGP554 $\rightarrow$ GP427 & \\
\hline GP412 & $\begin{array}{l}\operatorname{trp} C 2 \text { amyE::( } \triangle \mathrm{LA} p t s G^{\prime}-' l a c Z \\
\text { aphA3) } \Delta g l c T 8\end{array}$ & pGP342 $\rightarrow$ GP109 & \\
\hline GP413 & $\begin{array}{l}\operatorname{trpC} 2 \text { amyE::( }\left(\mathrm{LA}_{\mathrm{R} 2} p t s G^{\prime}-' l a c Z\right. \\
\text { aphA3) }\end{array}$ & pGP550 $\rightarrow$ GP168 & Schilling et al., 2006 \\
\hline GP414 & $\begin{array}{l}\operatorname{trp} C 2 \text { amyE::( }\left(\Delta \mathrm{LA}_{\mathrm{A} 4-\text {-ins }} \mathrm{U} 18 \mathrm{G}\right. \\
\text { pts } G^{\prime}-' \text { 'lacZ } \text { aphA3) }\end{array}$ & pGP551 $\rightarrow$ GP168 & \\
\hline GP415 & $\begin{array}{l}\operatorname{trpC} 2 \text { amyE::( }\left(\mathrm{LA}_{\mathrm{R} 3} p t s G^{\prime}-{ }^{\prime} l a c Z\right. \\
\text { aphA3) }\end{array}$ & pGP552 $\rightarrow$ GP168 & Schilling et al., 2006 \\
\hline GP416 & $\begin{array}{l}\operatorname{trp} C 2 \text { amyE::( }\left(\mathrm{LA}_{\mathrm{R} 4} p t s G^{\prime}-' l a c Z\right. \\
\text { aphA3) }\end{array}$ & pGP553 $\rightarrow$ GP168 & Schilling et al., 2006 \\
\hline GP417 & $\begin{array}{l}\operatorname{trp} C 2 \text { amyE::( }\left(\mathrm{LA}_{\mathrm{R} 4} p t s G^{\prime}-{ }^{\prime} l a c Z\right. \\
\text { aphA3) } \Delta l i c T S:: e r m\end{array}$ & pGP553 $\rightarrow$ GP427 & Schilling et al., 2006 \\
\hline GP418 & 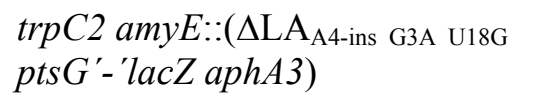 & pGP555 $\rightarrow$ GP168 & \\
\hline GP419 & $\begin{array}{l}\operatorname{trpC} 2 \text { amyE::( }\left(\mathrm{LA}_{\mathrm{R} 8} p t s G^{\prime}-{ }^{\prime} l a c Z\right. \\
\text { aphA3) }\end{array}$ & pGP561 $\rightarrow$ GP168 & Schilling et al., 2006 \\
\hline GP420 & $\begin{array}{l}\operatorname{trp} C 2 \text { amyE::( } \Delta \mathrm{LA}_{\mathrm{R} 8} p t s G^{\prime}-{ }^{\prime} l a c Z \\
\text { aphA3) } \Delta g l c T 8\end{array}$ & pGP561 $\rightarrow$ GP150 & Schilling et al., 2006 \\
\hline GP421 & $\begin{array}{l}\operatorname{trp} C 2 \text { amyE::( }\left(\Delta \mathrm{LA}_{\mathrm{R} 8} \text { pts } G^{\prime}-' l a c Z\right. \\
\text { aphA3) } \Delta l i c T S:: e r m\end{array}$ & pGP561 $\rightarrow$ GP427 & Schilling et al., 2006 \\
\hline GP422 & $\begin{array}{l}\operatorname{trp} C 2 \text { amyE::( } \triangle L A \text { pts } G \text { '- 'lacZ } \\
\text { neo) }\end{array}$ & pGP562 $\rightarrow 168$ & \\
\hline GP425 & $\operatorname{trp} C 2 \Delta s a c Y:: c a t$ & Long Flanking $\rightarrow 168$ & Schilling et al., 2006 \\
\hline GP427 & trpC2 $\Delta$ licTS::erm & $\begin{array}{l}\text { BGW10 chrom. } \rightarrow \\
168\end{array}$ & Schilling et al., 2006 \\
\hline GP429 & $\operatorname{trpC2} \Delta$ sacT::spec & $\begin{array}{l}\text { Long Flanking } \rightarrow \\
168\end{array}$ & Schilling et al., 2006 \\
\hline GP430 & $\operatorname{trp} C 2 \Delta s a c Y:: c a t \Delta s a c T:: s p e c$ & $\begin{array}{l}\text { GP429 chrom. } \rightarrow \\
\text { GP425 }\end{array}$ & Schilling et al., 2006 \\
\hline GP431 & $\operatorname{trp} C 2 \Delta l i c T S:: e r m \Delta$ sacT::spec & $\begin{array}{l}\text { GP429 chrom. } \rightarrow \\
\text { GP427 }\end{array}$ & Schilling et al., 2006 \\
\hline
\end{tabular}




\begin{tabular}{|c|c|c|c|}
\hline Name & Genotype & Construction $^{\text {a }}$ & Reference \\
\hline GP432 & $\operatorname{trp} C 2 \Delta$ licTS::erm $\Delta s a c Y:: c a t$ & $\begin{array}{l}\text { GP425 chrom. } \rightarrow \\
\text { GP427 }\end{array}$ & Schilling et al., 2006 \\
\hline GP433 & $\begin{array}{l}\text { trpC2 } \Delta \text { licTS::erm } \triangle \text { sac } Y:: c a t \\
\text { sacT::spec }\end{array}$ & $\begin{array}{l}\text { GP429 chrom. } \rightarrow \\
\text { GP432 }\end{array}$ & Schilling et al., 2006 \\
\hline GP434 & 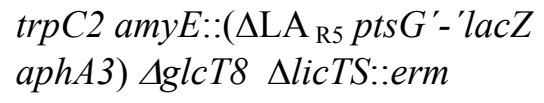 & $\begin{array}{l}\text { BGW10 chrom. } \rightarrow \\
\text { GP400 }\end{array}$ & Schilling et al., 2006 \\
\hline GP435 & $\begin{array}{l}\operatorname{trp} C 2 \Delta s a c Y:: c a t \text { amyE }::\left(\Delta \mathrm{LA}_{\mathrm{R} 5}\right. \\
\text { pts } G^{\prime}-{ }^{\prime} l a c Z \text { aphA3) } \Delta g l c T 8\end{array}$ & $\begin{array}{l}\text { GP425 chrom. } \rightarrow \\
\text { GP400 }\end{array}$ & Schilling et al., 2006 \\
\hline GP436 & $\begin{array}{l}\operatorname{trp} C 2 \text { amyE::( } \Delta \mathrm{LA}_{\mathrm{R} 5} p t s G^{\prime}-l a c Z \\
\text { aphA3) } \Delta g l c T 8 \Delta s a c T:: \mathrm{spec}\end{array}$ & $\begin{array}{l}\text { GP429 chrom. } \rightarrow \\
\text { GP400 }\end{array}$ & Schilling et al., 2006 \\
\hline GP437 & $\begin{array}{l}\operatorname{trp} C 2 \text { amyE: }:(\Delta \mathrm{LA} s a c B \text { '- 'lacZ } \\
\text { aphA3) }\end{array}$ & pGP564 $\rightarrow 168$ & Schilling et al., 2006 \\
\hline GP438 & 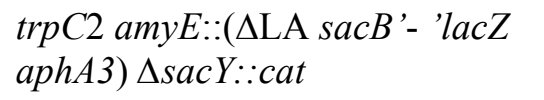 & pGP564 $\rightarrow$ GP425 & Schilling et al., 2006 \\
\hline GP439 & 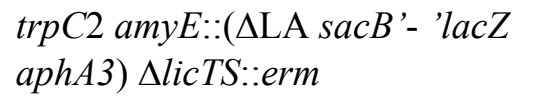 & pGP564 $\rightarrow$ GP427 & \\
\hline GP440 & $\begin{array}{l}\operatorname{trpC} 2 \text { amyE::( }(\mathrm{LA} \text { sacB' - 'lacZ } \\
\text { aphA3) } \Delta s a c T:: \text { spec }\end{array}$ & pGP564 $\rightarrow$ GP49 & Schilling et al., 2006 \\
\hline GP441 & 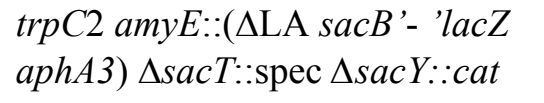 & pGP564 $\rightarrow$ GP430 & Schilling et al., 2006 \\
\hline GP442 & $\begin{array}{l}\operatorname{trp} C 2 \text { amyE::( } \Delta \text { LA sacB'- 'lacZ } \\
\text { aphA3) sacB } \Delta \text { licTS::erm } \Delta \\
\text { sacT::spec }\end{array}$ & pGP564 $\rightarrow$ GP431 & \\
\hline GP443 & $\begin{array}{l}\operatorname{trp} C 2 \text { amyE::( } \Delta \text { LA sacB'- 'lacZ } \\
\text { aphA3) sacB } \Delta l i c T S:: e r m \\
\Delta s a c Y:: \text { cat }\end{array}$ & pGP564 $\rightarrow$ GP432 & \\
\hline GP444 & $\begin{array}{l}\operatorname{trp} C 2 \text { amyE: :( }\left(\Delta \mathrm{LA}_{\mathrm{R} 10} s a c B \text { '- }\right. \\
\text { 'lacZ aphA3) }\end{array}$ & pGP565 $\rightarrow 168$ & Schilling et al., 2006 \\
\hline GP445 & 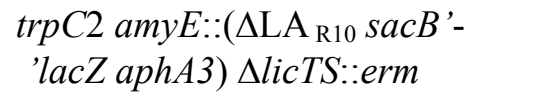 & pGP565 $\rightarrow$ GP427 & \\
\hline GP446 & $\begin{array}{l}\operatorname{trpC} 2 \text { amyE::( }\left(\Delta \mathrm{LA}_{\mathrm{R} 11} s a c B \text { '- }\right. \\
\text { 'lacZ aphA3) }\end{array}$ & pGP566 $\rightarrow 168$ & Schilling et al., 2006 \\
\hline GP447 & 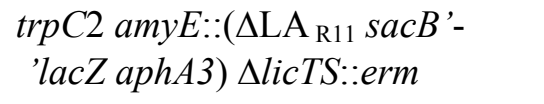 & pGP566 $\rightarrow$ GP427 & \\
\hline GP448 & $\begin{array}{l}\operatorname{trpC} 2 \text { amyE::( }\left(\Delta \mathrm{LA}_{\mathrm{R} 12} s a c B \text { '- }\right. \\
\text { 'lacZ aphA3) }\end{array}$ & pGP567 $\rightarrow 168$ & Schilling et al., 2006 \\
\hline GP449 & $\begin{array}{l}\operatorname{trp} C 2 \text { amyE::( }\left(\Delta \mathrm{LA}_{\mathrm{R} 12} s a c B \text { '- }\right. \\
\text { 'lacZ aphA3) } \Delta l i c T S\end{array}$ & pGP567 $\rightarrow$ GP427 & \\
\hline GP450 & $\begin{array}{l}\operatorname{trpC} 2 \text { amyE::( }\left(\Delta \mathrm{LA}_{\mathrm{R} 13} s a c B \text { '- }\right. \\
\text { 'lacZ aphA3) }\end{array}$ & pGP568 $\rightarrow 168$ & Schilling et al., 2006 \\
\hline GP451 & $\begin{array}{l}\operatorname{trp} C 2 \text { amyE::( } \Delta \mathrm{LA}_{\mathrm{R} 13} s a c B '- \\
\quad \text { 'lacZ aphA3) } \Delta g l c T 8\end{array}$ & pGP568 $\rightarrow$ GP150 & Schilling et al., 2006 \\
\hline
\end{tabular}




\begin{tabular}{|c|c|c|c|}
\hline Name & Genotype & Construction $^{\text {a }}$ & Reference \\
\hline GP452 & $\begin{array}{l}\operatorname{trp} C 2 \text { amyE::( } \Delta \mathrm{LA}_{\mathrm{R} 13} s a c B '- \\
\text { 'lacZ aphA3) } \Delta l i c T S\end{array}$ & pGP568 $\rightarrow$ GP427 & \\
\hline GP453 & $\begin{array}{l}\operatorname{trp} C 2 \text { amyE::( } \Delta \mathrm{LA}_{\mathrm{R} 13} \text { sacB'- } \\
\text { 'lacZ aphA3) } \Delta \text { licTS::erm } \\
\Delta \text { sacY::cat sacT::spec }\end{array}$ & pGP568 $\rightarrow$ GP433 & Schilling et al., 2006 \\
\hline GP454 & $\begin{array}{l}\operatorname{trp} C 2 \text { amyE::(}\left(\Delta \mathrm{LA}_{\mathrm{R} 5} \text { pts } G^{\prime}-{ }^{\prime} l a c Z\right. \\
\text { aphA3) } \Delta l i c T S:: \operatorname{erm} \Delta \text { sacT::spec }\end{array}$ & pGP557 $\rightarrow$ GP431 & Schilling et al., 2006 \\
\hline GP455 & $\begin{array}{l}\operatorname{trp} C 2 \text { amyE::( } \Delta \mathrm{LA}_{\mathrm{R} 5} \text { pts } G^{\prime}-{ }^{\prime} l a c Z \\
\text { aphA3) } \Delta l i c T S:: e r m \Delta s a c Y:: c a t\end{array}$ & pGP557 $\rightarrow$ GP432 & Schilling et al., 2006 \\
\hline GP456 & $\begin{array}{l}\operatorname{trpC2} \text { amyE::(}\left(\Delta \mathrm{LA}_{\mathrm{R} 5} \text { ptsG'-'lacZ }\right. \\
\text { aphA3) } \Delta \text { licTS::erm } \Delta \text { sacY::cat } \\
\text { sacT::spec }\end{array}$ & pGP557 $\rightarrow$ GP433 & Schilling et al., 2006 \\
\hline GP457 & $\operatorname{trp} C 2 \Delta y d a p:: s p e c$ & LFH $\rightarrow 168$ & \\
\hline GP458 & $\begin{array}{l}\operatorname{trp} C 2 \text { amyE::(citB- 'lacZ cat) } \\
\Delta y d a p:: s p e c \triangle \text { Dta::aphA3 }\end{array}$ & $\mathrm{LFH} \rightarrow \mathrm{GP} 241$ & \\
\hline GP459 & $\operatorname{trp} C 2 \Delta p d h B:: s p e c$ & pGP579 $\rightarrow 168$ & \\
\hline GP460 & $\begin{array}{l}\operatorname{trp} C 2 \text { amyE::( }\left(\Delta \mathrm{LA}_{\mathrm{R} 2} s a c B \text { '- }\right. \\
\text { 'lacZ aphA3) }\end{array}$ & pGP569 $\rightarrow 168$ & Schilling et al., 2006 \\
\hline GP461 & $\begin{array}{l}\operatorname{trp} C 2 \text { amyE::( }\left(\Delta \mathrm{LA}_{\mathrm{R} 1} s a c B \text { '- }\right. \\
\text { 'lacZ aphA3) }\end{array}$ & pGP580 $\rightarrow 168$ & Schilling et al., 2006 \\
\hline GP462 & $\begin{array}{l}\operatorname{trp} C 2 \text { amyE::( } \Delta \mathrm{LA}_{\mathrm{R} 1} \text { sacB'- } \\
\text { 'lacZ aphA3) } \Delta \text { licTS::erm }\end{array}$ & pGP580 $\rightarrow$ GP427 & Schilling et al., 2006 \\
\hline GP463 & $\begin{array}{l}\operatorname{trp} C 2 \text { amyE::( } \Delta \mathrm{LA}_{\mathrm{R} 1} \text { sacB' } \\
\quad \text { 'lacZ aphA3) } \Delta \text { sacY::cat } \\
\text { sacT::spec }\end{array}$ & pGP580 $\rightarrow$ GP430 & Schilling et al., 2006 \\
\hline GP464 & $\begin{array}{l}\operatorname{trp} C 2 \text { amyE::( }\left(\Delta \mathrm{LA}_{\mathrm{R} 7} p t s G^{\prime}-{ }^{\prime} l a c Z\right. \\
\text { aphA3) }\end{array}$ & pGP591 $\rightarrow 168$ & Schilling et al., 2006 \\
\hline GP465 & $\begin{array}{l}\operatorname{trp} C 2 \text { amyE::( } \triangle \mathrm{LA}_{\mathrm{R} 1} \text { sacB'- } \\
\quad \text { 'lacZ aphA3) } \Delta \text { licTS::erm } \\
\text { sacT::spec }\end{array}$ & pGP580 $\rightarrow 431$ & Schilling et al., 2006 \\
\hline GP466 & $\begin{array}{l}\operatorname{trp} C 2 \text { amyE::(}\left(\Delta \mathrm{LA}_{\mathrm{R} 1} \operatorname{sacB}{ }^{\prime}-\right. \\
\quad \text { 'lacZ aphA3) } \Delta \text { licTS::erm } \\
\Delta \text { sacY::cat }\end{array}$ & pGP580 $\rightarrow 432$ & Schilling et al., 2006 \\
\hline GP467 & $\begin{array}{l}\operatorname{trp} C 2 \text { amyE::( }\left(\Delta \mathrm{LA}_{\mathrm{R} 1} s a c B '-\right. \\
\text { 'lacZ aphA3) } \triangle g l c T 8\end{array}$ & pGP580 $\rightarrow$ GP150 & \\
\hline GP468 & $\begin{array}{l}\operatorname{trp} C 2 \text { amyE::( }\left(\Delta \mathrm{LA}_{\mathrm{R} 5} \text { pts } G^{\prime}-{ }^{\prime} l a c Z\right. \\
\text { aphA3) } \Delta \text { sac } Y:: \text { cat } \Delta \text { sacT }:: \text { spec }\end{array}$ & pGP557 $\rightarrow$ GP430 & \\
\hline GP469 & $\operatorname{trp} C 2 \Delta c s r A:: s p e c$ & $\mathrm{LFH} \rightarrow 168$ & \\
\hline GP470 & $\begin{array}{l}\operatorname{trp} C 2 \Delta p t s G:: \text { cat } \triangle b g l P:: \operatorname{Tn} 10 \\
\text { erm }\end{array}$ & $\begin{array}{l}\text { chrom. DNA } \\
\text { GM1112 } \rightarrow \text { QB5435 }\end{array}$ & Schilling et al., 2006 \\
\hline GP471 & $\begin{array}{l}\operatorname{trp} C 2 \text { amyE::( }\left(\Delta \mathrm{LA}_{\mathrm{R} 2} \text { sacB' }\right. \\
\quad \text { 'lacZ aphA3) } \Delta \text { licTS::erm } \\
\text { sacT::spec }\end{array}$ & pGP569 $\rightarrow$ GP431 & Schilling et al., 2006 \\
\hline
\end{tabular}




\begin{tabular}{|c|c|c|c|}
\hline Name & Genotype & Construction $^{\text {a }}$ & Reference \\
\hline GP472 & $\begin{array}{l}\operatorname{trp} C 2 \text { amyE::( } \Delta \mathrm{LA}_{\mathrm{R} 2} s a c B '- \\
\quad \text { 'lacZ aphA3) } \Delta \text { licTS::erm } \\
\Delta \text { sacY::cat }\end{array}$ & pGP569 $\rightarrow$ GP 432 & Schilling et al., 2006 \\
\hline GP473 & $\begin{array}{l}\operatorname{trp} C 2 \text { amyE::( }\left(\mathrm{LA}_{\mathrm{R} 2} \text { sacB' }\right. \\
\text { 'lacZ aphA3) } \Delta \text { licTS::erm }\end{array}$ & pGP569 $\rightarrow$ GP427 & \\
\hline GP474 & $\operatorname{trp} C 2 \Delta p t s G:: c a t$ & $\begin{array}{l}\text { chrom. DNA } \\
\text { QB5449 } \rightarrow 168\end{array}$ & \\
\hline GP475 & $\operatorname{trp} C 2 \Delta b g l P:: \mathrm{erm}$ & $\begin{array}{l}\text { chrom. DNA } \\
\text { GM1112 } \rightarrow 168\end{array}$ & \\
\hline GP476 & $\begin{array}{l}\operatorname{trp} C 2 \text { amyE::( }\left(\Delta \mathrm{LA}_{\mathrm{R} 7} s a c B \text { '- }\right. \\
\text { 'lacZ aph } A 3)\end{array}$ & pGP582 $\rightarrow 168$ & Schilling et al., 2006 \\
\hline GP477 & $\begin{array}{l}\operatorname{trp} C 2 \text { amyE::( }\left(\Delta \mathrm{LA}_{\mathrm{R} 8} \text { sacB'- }\right. \\
\text { 'lacZ aph } A 3)\end{array}$ & pGP587 $\rightarrow 168$ & Schilling et al., 2006 \\
\hline GP478 & $\begin{array}{l}\operatorname{trpC2} \text { amyE::( }\left(\Delta \mathrm{LA}_{\mathrm{G} 26 \mathrm{~A}} \operatorname{sacB}{ }^{\prime}-\right. \\
\text { 'lacZ aphA3) }\end{array}$ & pGP581 $\rightarrow 168$ & \\
\hline GP479 & $\begin{array}{l}\operatorname{trp} C 2 \text { amyE::( } \triangle \mathrm{LA}_{\mathrm{A} 13 \mathrm{C}} \mathrm{U} 14 \mathrm{G} \mathrm{G} 26 \mathrm{~A} \\
\operatorname{sacB} \text { '- 'lacZ aphA3) }\end{array}$ & pGP586 $\rightarrow 168$ & \\
\hline GP480 & $\begin{array}{l}\operatorname{trp} C 2 \text { amyE::( }\left(\triangle \mathrm{LA}_{\mathrm{R} 9} \text { sacB'- }\right. \\
\text { 'lacZ aphA3) }\end{array}$ & pGP583 $\rightarrow 168$ & Schilling et al., 2006 \\
\hline GP481 & 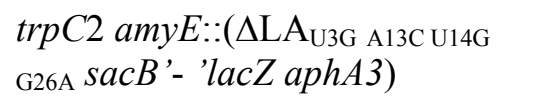 & pGP588 $\rightarrow 168$ & \\
\hline GP482 & $\begin{array}{l}\operatorname{trp} C 2 \text { amyE::( } \triangle \mathrm{LA}_{\mathrm{A} 13 \mathrm{C}} \mathrm{U} 14 \mathrm{G} \\
\operatorname{sacB} \text { '- 'lacZ aphA3) }\end{array}$ & pGP584 $\rightarrow 168$ & \\
\hline GP483 & $\begin{array}{l}\operatorname{trp} C 2 \text { amyE::( } \triangle \mathrm{LA}_{\mathrm{U} 3 \mathrm{G}} \mathrm{A} 13 \mathrm{C} \mathrm{U} 14 \mathrm{G} \\
\operatorname{sacB} \text { '- 'lacZ aphA3) }\end{array}$ & pGP585 $\rightarrow 168$ & \\
\hline GP484 & $\begin{array}{l}\operatorname{trp} C 2 \text { amyE::( } \Delta \mathrm{LA}_{\mathrm{R} 7} \text { sacB'- } \\
\text { 'lacZ aphA3) } \Delta \text { licTS ::erm }\end{array}$ & pGP582 $\rightarrow$ GP427 & Schilling et al., 2006 \\
\hline GP485 & $\begin{array}{l}\operatorname{trp} C 2 \text { amyE::( } \triangle \mathrm{LA}_{\mathrm{R} 7} \text { sacB'- } \\
\quad \text { 'lacZ aphA3) } \Delta \text { sacY::cat } \\
\text { sacT::spec }\end{array}$ & pGP582 $\rightarrow$ GP430 & \\
\hline GP486 & $\begin{array}{l}\operatorname{trp} C 2 \text { amyE::( }\left(\mathrm{LA}_{\mathrm{R} 8} \text { sacB'- }\right. \\
\text { 'lacZ aphA3) } \Delta \text { licTS::erm }\end{array}$ & pGP587 $\rightarrow$ GP427 & Schilling et al., 2006 \\
\hline GP487 & $\begin{array}{l}\operatorname{trp} C 2 \text { amyE:: }\left(\Delta \mathrm{LA}_{\mathrm{R} 8} \operatorname{sacB} \text { '- 'lacZ }\right. \\
\text { aphA3) sacT::spec }\end{array}$ & pGP587 $\rightarrow$ GP429 & Schilling et al., 2006 \\
\hline GP488 & $\begin{array}{l}\operatorname{trpC2} \text { amyE::( }\left(\Delta \mathrm{LA}_{\mathrm{G} 26 \mathrm{~A}} \operatorname{sacB}{ }^{\prime}-\right. \\
\text { 'lacZ aphA3) sacT::spec }\end{array}$ & pGP581 $\rightarrow$ GP429 & \\
\hline GP489 & $\begin{array}{l}\operatorname{trp} C 2 \text { amyE::( } \Delta \mathrm{LA}_{\mathrm{G} 26 \mathrm{~A}} \text { sacB'- } \\
\text { 'lacZ aphA3) } \Delta \text { sacY::cat }\end{array}$ & pGP581 $\rightarrow$ GP425 & \\
\hline GP490 & $\begin{array}{l}\operatorname{trp} C 2 \text { amyE::( } \triangle \mathrm{LA}_{\mathrm{A} 13 \mathrm{C}} \mathrm{U} 14 \mathrm{G} \mathrm{G} 26 \mathrm{~A} \\
\text { sacB'- 'lacZ aphA3) sacT::spec }\end{array}$ & pGP586 $\rightarrow$ GP429 & \\
\hline GP491 & $\begin{array}{l}\operatorname{trp} C 2 \text { amyE::( } \triangle \mathrm{LA}_{\mathrm{A} 13 \mathrm{C}} \mathrm{U} 14 \mathrm{G} \mathrm{G} 26 \mathrm{~A} \\
\operatorname{sacB} \text { '- 'lacZ aphA3) } \Delta \text { sacY::cat }\end{array}$ & pGP586 $\rightarrow$ GP425 & \\
\hline GP492 & $\begin{array}{l}\operatorname{trp} C 2 \text { amyE::( }\left(\Delta \mathrm{LA}_{\mathrm{R} 9} \text { sacB'- }\right. \\
\text { 'lacZ aphA3) } \Delta \text { licTS::erm }\end{array}$ & pGP583 $\rightarrow$ GP427 & Schilling et al., 2006 \\
\hline
\end{tabular}




\begin{tabular}{|c|c|c|c|}
\hline Name & Genotype & Construction $^{\text {a }}$ & Reference \\
\hline GP493 & $\begin{array}{l}\operatorname{trp} C 2 \text { amyE::( } \Delta \mathrm{LA}_{\mathrm{R} 9} \text { sacB'- } \\
\quad \text { 'lacZ aphA3) } \Delta l i c T S:: e r m \\
\text { sacT::spec }\end{array}$ & pGP583 $\rightarrow$ GP431 & Schilling et al., 2006 \\
\hline GP494 & $\begin{array}{l}\operatorname{trp} C 2 \text { amyE::( }\left(\mathrm{LA}_{\mathrm{R} 9} \text { sacB'- }\right. \\
\text { 'lacZ aphA3) } \Delta \text { licTS::erm } \\
\Delta \text { sacY::cat }\end{array}$ & pGP583 $\rightarrow$ GP432 & Schilling et al., 2006 \\
\hline GP495 & $\begin{array}{l}\operatorname{trp} C 2 \text { amyE::( }\left(\mathrm{LA}_{\mathrm{R} 9} \text { sacB'- }\right. \\
\text { 'lacZ aphA3) sacT::spec } \\
\Delta \text { sacY::cat }\end{array}$ & pGP583 $\rightarrow$ GP430 & \\
\hline GP496 & 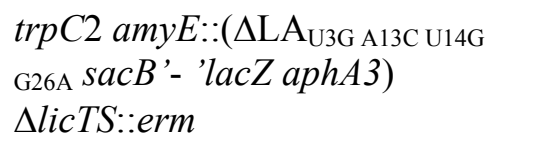 & pGP588 $\rightarrow$ GP427 & \\
\hline GP497 & $\begin{array}{l}\operatorname{trp} C 2 \text { amyE::( }\left(\mathrm{LA}_{\mathrm{U} 3 \mathrm{G}} \mathrm{A} 13 \mathrm{C} \mathrm{U} 14 \mathrm{G}\right. \\
\text { G26A } \operatorname{sacB} \text { '- 'lacZ aphA3) } \\
\Delta \text { sacT }:: \text { spec }\end{array}$ & pGP588 $\rightarrow$ GP429 & \\
\hline GP498 & $\begin{array}{l}\operatorname{trp} C 2 \text { amyE::( }\left(\Delta \mathrm{LA}_{\mathrm{A} 13 \mathrm{C}} \mathrm{U} 14 \mathrm{G} s a c B '-\right. \\
\text { 'lacZ aphA3) } \Delta s a c T:: s p e c\end{array}$ & pGP584 $\rightarrow$ GP429 & \\
\hline GP499 & 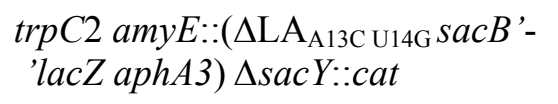 & pGP584 $\rightarrow$ GP425 & \\
\hline GP518 & $\begin{array}{l}\operatorname{trpC2} \text { amyE::( } \Delta \mathrm{LA}_{\mathrm{A} 13 \mathrm{C}} \mathrm{U} 14 \mathrm{G} \mathrm{G} 26 \mathrm{~A} \\
\text { sacB'- 'lacZ aphA3) } \Delta l i c T S:: e r m \\
\Delta \text { sacY }:: \text { cat } \Delta \text { sacT::spec }\end{array}$ & pGP586 $\rightarrow$ GP433 & \\
\hline GP519 & $\begin{array}{l}\operatorname{trp} C 2 \text { amyE::( }\left(\mathrm{LA}_{\mathrm{R} 3} s a c B \text { '- 'lacZ }\right. \\
\text { aphA3) }\end{array}$ & pGP589 $\rightarrow 168$ & Schilling et al., 2006 \\
\hline GP520 & $\begin{array}{l}\operatorname{trp} C 2 \text { amyE::( }\left(\mathrm{LA}_{\mathrm{R} 5} \operatorname{sacB}{ }^{\prime}-{ }^{\prime} l a c Z\right. \\
\text { aphA3) }\end{array}$ & pGP590 $\rightarrow 168$ & Schilling et al., 2006 \\
\hline GP521 & $\begin{array}{l}\operatorname{trp} C 2 \text { amyE::( }\left(\Delta \mathrm{LA}_{\mathrm{R} 3} s a c B \text { '- 'lacZ }\right. \\
\text { aphA3) } \Delta \text { sacT::spec }\end{array}$ & pGP589 $\rightarrow$ GP429 & Schilling et al., 2006 \\
\hline GP522 & $\begin{array}{l}\operatorname{trp} C 2 \text { amyE::( }\left(\Delta \mathrm{LA}_{\mathrm{R} 5} \operatorname{sacB} \text { '- 'lacZ }\right. \\
\text { aphA3) } \Delta \text { sacT::spec }\end{array}$ & pGP590 $\rightarrow$ GP429 & Schilling et al., 2006 \\
\hline GP523 & $\begin{array}{l}\operatorname{trp} C 2 \text { amyE::( }\left(\mathrm{LA}_{\mathrm{R} 3} \operatorname{sacB} \text { '- 'lacZ }\right. \\
\text { aphA3) } \Delta \text { sac } Y:: c a t\end{array}$ & pGP589 $\rightarrow$ GP429 & \\
\hline GP524 & $\begin{array}{l}\operatorname{trp} C 2 \text { amyE::( }\left(\Delta \mathrm{LA}_{\mathrm{R} 5} \operatorname{sacB} \text { '- 'lacZ }\right. \\
\text { aphA3) } \Delta \text { sacY::cat }\end{array}$ & pGP590 $\rightarrow$ GP425 & \\
\hline GP525 & $\begin{array}{l}\operatorname{trp} C 2 \text { amyE::( } \triangle \mathrm{LA}_{\mathrm{A} 4-\mathrm{ins}} \mathrm{A} 4 \mathrm{U} \text { U14del } \\
\text { C15A G16A U18G U25A A88U } p t s G^{\prime}-{ }^{\prime} l a c Z \\
\text { aphA3) }\end{array}$ & pGP592 $\rightarrow 168$ & \\
\hline GP526 & 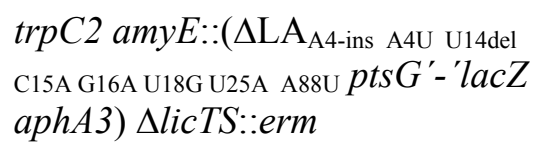 & pGP592 $\rightarrow$ GP427 & \\
\hline GP527 & $\begin{array}{l}\operatorname{trp} C 2 \text { amyE::( } \triangle \mathrm{LA}_{\mathrm{A} 4-\mathrm{ins}} \mathrm{A} 4 \mathrm{U} \text { U14del } \\
\text { C15A G16A U18G U25A A88U } p t s G^{\prime}-{ }^{\prime} l a c Z \\
\text { aphA3) } \Delta s a c T:: s p e c\end{array}$ & pGP592 $\rightarrow$ GP429 & \\
\hline GP528 & $\begin{array}{l}\operatorname{trp} C 2 \text { amyE }::\left(\Delta \mathrm{LA}_{\mathrm{R} 5} p t s G^{\prime}-{ }^{\prime} l a c Z\right. \\
\text { aphA3) } \Delta p t s G:: c a t\end{array}$ & pGP557 $\rightarrow$ GP474 & \\
\hline
\end{tabular}




\begin{tabular}{|c|c|c|c|}
\hline Name & Genotype & Construction $^{\text {a }}$ & Reference \\
\hline GP529 & $\begin{array}{l}\operatorname{trp} C 2 \text { amyE::( }\left(\Delta \mathrm{LA}_{\mathrm{R} 5} p t s G^{\prime}-{ }^{\prime} l a c Z\right. \\
\text { aphA3) } \Delta p t s G:: c a t\end{array}$ & pGP557 $\rightarrow$ QB5435 & \\
\hline GP530 & $\begin{array}{l}\operatorname{trp} C 2 \text { amyE::( }\left(\Delta \mathrm{LA}_{\mathrm{R} 5} p t s G^{\prime}-{ }^{\prime} l a c Z\right. \\
\text { aphA3) } \Delta l i c T S:: \mathrm{erm}\end{array}$ & pGP557 $\rightarrow$ GP473 & \\
\hline GP531 & 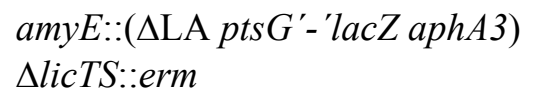 & pGP66 $\rightarrow$ GP473 & \\
\hline GP532 & $\begin{array}{l}\operatorname{trp} C 2 \text { amyE::( } \Delta \mathrm{LA}_{\mathrm{R} 9} s a c B '- \\
\text { 'lacZ aphA3) } \Delta g l c T 8\end{array}$ & pGP583 $\rightarrow$ GP150 & \\
\hline GP533 & 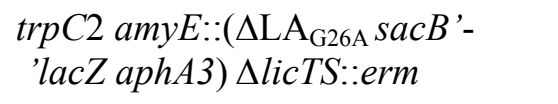 & pGP581 $\rightarrow$ GP427 & \\
\hline GP534 & 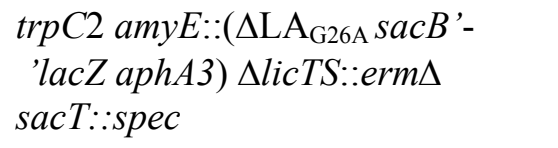 & pGP581 $\rightarrow$ GP431 & \\
\hline GP535 & $\begin{array}{l}\operatorname{trp} C 2 \text { amyE::( }\left(\Delta \mathrm{LA}_{\mathrm{G} 26 \mathrm{~A}} \operatorname{sacB}{ }^{\prime}-\right. \\
\text { 'lacZ aphA3) } \Delta \text { licTS::erm } \\
\Delta \text { sacY::cat }\end{array}$ & pGP581 $\rightarrow$ GP432 & \\
\hline GP536 & $\begin{array}{l}\operatorname{trp} C 2 \text { amyE::( }\left(\Delta \mathrm{LA}_{\mathrm{R} 7} \operatorname{sacB}{ }^{\prime}-\right. \\
\quad \text { 'lacZ aphA3) } \Delta \text { licTS::erm } \Delta \\
\text { sacT::spec }\end{array}$ & pGP582 $\rightarrow$ GP431 & Schilling et al., 2006 \\
\hline GP537 & $\begin{array}{l}\operatorname{trp} C 2 \text { amyE::( }\left(\Delta \mathrm{LA}_{\mathrm{R} 6} \operatorname{sacB}^{\prime}-\right. \\
\text { 'lacZ aphA3) }\end{array}$ & pGP595 $\rightarrow$ GP168 & Schilling et al., 2006 \\
\hline GP538 & $\begin{array}{l}\operatorname{trp} C 2 \text { amyE::( }\left(\Delta \mathrm{LA}_{\mathrm{R} 6} s a c B \text { '- 'lacZ }\right. \\
\text { aphA3) } \Delta \text { sacT::spec }\end{array}$ & pGP595 $\rightarrow$ GP429 & Schilling et al., 2006 \\
\hline GP539 & $\begin{array}{l}\operatorname{trp} C 2 \text { amyE::( }\left(\Delta \mathrm{LA}_{\mathrm{R} 6} \operatorname{sacB} \text { '- 'lacZ }\right. \\
\text { aphA3) sacT::spec } \Delta \text { sacY::cat }\end{array}$ & pGP595 $\rightarrow$ GP430 & Schilling et al., 2006 \\
\hline GP540 & $\begin{array}{l}\operatorname{trp} C 2 \text { amyE::( }\left(\mathrm{LA}_{\mathrm{R} 4} s a c B \text { '- 'lacZ }\right. \\
\text { aphA3) }\end{array}$ & pGP596 $\rightarrow$ GP168 & Schilling et al., 2006 \\
\hline GP541 & $\begin{array}{l}\operatorname{trp} C 2 \text { amyE::( }\left(\mathrm{LA}_{\mathrm{R} 4} s a c B \text { '- 'lacZ }\right. \\
\text { aphA3) } \Delta \text { sacT::spec }\end{array}$ & pGP596 $\rightarrow$ GP429 & Schilling et al., 2006 \\
\hline GP542 & $\begin{array}{l}\operatorname{trp} C 2 \text { amyE::( }\left(\Delta \mathrm{LA}_{\mathrm{R} 4} \operatorname{sacB} \text { '- 'lacZ }\right. \\
\text { aphA3) sacT::spec } \Delta \text { sacY::cat }\end{array}$ & pGP596 $\rightarrow$ GP430 & Schilling et al., 2006 \\
\hline GP543 & $\begin{array}{l}\operatorname{trpC} 2 \text { amyE::( } \Delta \mathrm{LA}_{\mathrm{R} 10} \text { sacB'- } \\
\text { 'lacZ aphA3) sacT::spec }\end{array}$ & pGP565 $\rightarrow$ GP429 & Schilling et al., 2006 \\
\hline GP544 & $\begin{array}{l}\text { trpC2 amyE::( }\left(\Delta \mathrm{LA}_{\mathrm{R} 7} \text { sacB'- }\right. \\
\text { 'lacZ aphA3) sacT::spec }\end{array}$ & pGP582 $\rightarrow$ GP429 & Schilling et al., 2006 \\
\hline GP545 & $\begin{array}{l}\operatorname{trp} C 2 \text { amyE::( }\left(\Delta \mathrm{LA}_{\mathrm{U} 3 \mathrm{G} \mathrm{A} 13 \mathrm{C} \mathrm{U} 14 \mathrm{G}}\right. \\
\text { sacB'- 'lacZ aphA3) } \Delta l i c T S:: \text { erm }\end{array}$ & pGP597 $\rightarrow$ GP427 & \\
\hline GP546 & $\begin{array}{l}\operatorname{trp} C 2 \text { amyE::( }\left(\mathrm{LA}_{\mathrm{U} 3 \mathrm{G}} \mathrm{A} 13 \mathrm{C} \mathrm{U} 14 \mathrm{G}\right. \\
\operatorname{sacB} \text { '- 'lacZ aphA3) } \Delta \text { sacT::spec }\end{array}$ & pGP597 $\rightarrow$ GP429 & \\
\hline GP547 & $\begin{array}{l}\operatorname{trp} C 2 \text { amyE::( }\left(\Delta \mathrm{LA}_{\mathrm{A} 4-\mathrm{ins}} \mathrm{G} 3 \mathrm{~A} A 4 \mathrm{U}\right. \\
\mathrm{U} 8 \mathrm{G} \text { U14del } \mathrm{C} 15 \mathrm{~A} \text { G16A U18G U25A A88U } \\
\left.p t s G^{\prime}-{ }^{\prime}-l a c Z \text { aph } A 3\right)\end{array}$ & pGP598 $\rightarrow$ GP168 & \\
\hline
\end{tabular}




\begin{tabular}{|c|c|c|c|}
\hline Name & Genotype & Construction $^{\text {a }}$ & Reference \\
\hline GP548 & $\begin{array}{l}\operatorname{trpC} 2 \text { amyE::( }\left(\Delta \mathrm{LA}_{\mathrm{A} 4-\mathrm{ins}} \mathrm{G} 3 \mathrm{U} \mathrm{A} 4 \mathrm{U}\right. \\
\mathrm{U} 14 \mathrm{del} \mathrm{C} 15 \mathrm{~A} \mathrm{G} 16 \mathrm{~A} \text { U18G U25A A88U } p t s G^{\prime}- \\
\text { 'lacZ aphA3) }\end{array}$ & pGP599 $\rightarrow$ GP168 & \\
\hline GP549 & 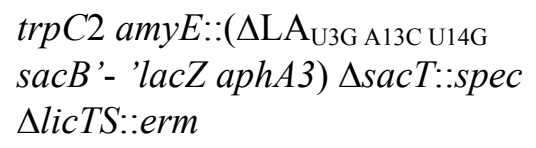 & pGP597 $\rightarrow$ GP430 & \\
\hline
\end{tabular}

${ }^{\mathrm{a}}$ Arrows indicate construction by transformation. 
Table 19. Regulation of genes encoding enzymes of the central metabolic pathways as determined by microarray analysis

\begin{tabular}{|c|c|c|c|c|}
\hline Gene & Operon & Description & Regulation factor a & Standard deviation $b$ \\
\hline \multicolumn{5}{|c|}{ Glycolysis } \\
\hline$p t s G$ & $p t s G H I$ & glucose-specific enzyme II & 0.99 & 0.071 \\
\hline$p t s H$ & $p t s G H I$ & $\mathrm{HPr}$ & 1.02 & 0.110 \\
\hline$p t s I$ & $p t s G H I$ & PTS enzyme I & 0.98 & 0.750 \\
\hline$p g i$ & pgi & phosphoglucoisomerase & 1.06 & 0.204 \\
\hline$p f k$ & pfk pykA & phosphofructokinase & 1.30 & 0.228 \\
\hline$f b a A$ & $f b a A$ & fructose-1,6-bisphosphate aldolase & 1.20 & 0.199 \\
\hline tpi & cggR gapA pgk tpiA pgm eno & triose phosphate isomerase & 1.23 & 0.128 \\
\hline gapA & cggR gapA pgk tpiA pgm eno & glyceraldehyde-3-phosphate dehydrogenase & 1.13 & 0.183 \\
\hline$p g k$ & cggR gapA pgk tpiA pgm eno & phosphoglycerate kinase & 1.25 & 0.125 \\
\hline pgm & cggR gapA pgk tpiA pgm eno & phosphoglycerate mutase & 1.19 & 0.167 \\
\hline eno & cggR gapA pgk tpiA pgm eno & enolase & 1.08 & 0.184 \\
\hline$p y k A$ & $p f k p y k A$ & pyruvate kinase & 1.29 & 0.175 \\
\hline \multicolumn{5}{|c|}{ Pyruvate dehydrogenase } \\
\hline$p d h A$ & $p d h A B C D$ & $\begin{array}{l}\text { pyruvate dehydrogenase (pyruvate decarboxylase, } \\
\text { subunit } \alpha \text { ) }\end{array}$ & 1.69 & 0.180 \\
\hline
\end{tabular}




$\begin{array}{ll}p d h B & p d h A B C D \\ p d h C & p d h A B C D \\ p d h D & p d h A B C D\end{array}$

\section{Fermentative and overflow metabolism}

$\begin{array}{ll}\text { alsS } & \text { als } S D \\ \text { alsD } & \text { alsSD } \\ \text { ldh } & \text { ldh lctP } \\ \text { pta } & \text { pta } \\ \text { ackA } & \text { ackA }\end{array}$

mleA

\section{Krebs acid cycle}

citA

citZ $\quad$ citZ icd $m d h$

icd $\quad$ citZ icd mdh

mdh $\quad$ citZ icd $m d h$

citB pyruvate dehydrogenase (pyruvate decarboxylase, subunit $\beta$ )

pyruvate dehydrogenase (dihydrolipoamide acetyltransferase)

pyruvate dehydrogenase (lipoamide dehydrogenase)

$\alpha$-acetolactate synthase

10.04

1.372

$\alpha$-acetolactate decarboxylase

1.039

lactate dehydrogenase

1.67

0.276

phosphotransacetylase

1.67

0.287

acetate kinase

2.94

0.650

malate oxidoreductase

citrate synthase (minor)

citrate synthase (major)

0.20

0.023

isocitrate dehydrogenase

0.55

0.033

malate dehydrogenase

0.62

0.057

aconitase 


$\begin{array}{ll}\text { odhA } & \text { odhAB } \\ \text { odhB } & \text { odhAB } \\ \text { sucC } & \text { sucCD } \\ \text { sucD } & \text { sucCD } \\ \text { sdhC } & \text { sdhCAB } \\ \text { sdhA } & \text { sdhCAB } \\ \text { sdhB } & \text { sdhCAB } \\ \text { citG } & \end{array}$

\section{Pentose phosphate pathway}

$\begin{array}{ll}z w f & \text { glucose-6-phosphate 1-dehydrogenase } \\ y q j I & \text { 6-phosphogluconate dehydrogenase } \\ r p e & \text { ribulose-5-phosphate epimerase } \\ y w l F & \text { ribose-5-phosphate isomerase (putative) } \\ t k t & \text { transketolase } \\ y w j H & \text { transaldolase (putative) }\end{array}$

\section{Gluconeogenesis}


pckA

mals

\section{Anaplerotic reaction}

pycA

Transporter of organic acids

$\begin{array}{ll}c i t M & \operatorname{citM}(y f l O) \\ c i t H & \operatorname{citH}(y x i Q) \\ d c t P & d c t P(y d b H) \\ g l t P & g l t P \\ g l t T & g l t T(y h f G) \\ g l n Q & g \ln Q H M P\end{array}$

\section{Nitrogen metabolism}

$\begin{array}{ll}\text { gltA } & \text { gltAB } \\ \text { gltB } & \text { gltAB } \\ \text { glnA } & \text { glnRA } \\ \operatorname{nrg} A & \operatorname{nrg} A B \\ \operatorname{rrg} B & \operatorname{nrg} A B \\ \operatorname{roc} G & \operatorname{roc} G\end{array}$

phosphoenolpyruvate carboxykinase

malate dehydrogenase (decarboxylating)

pyruvate carboxylase

$\mathrm{Mg}^{2+} /$ citrate transporter

1.13

0.382

$\mathrm{Ca}^{2+} /$ citrate transporter

1.21

0.332

C4-dicarboxylate transport protein

0.82

0.303

glutamate-aspartate carrier protein

1.03

0.343

$\mathrm{H}^{+} / \mathrm{Na}^{+}$-glutamate symport protein

glutamine $\mathrm{ABC}$ transporter

0.327

glutamate synthase (large subunit)

0.42

0.047

glutamate synthase (small subunit)

0.40

0.055

glutamine synthetase

0.67

0.060

ammonium transporter (AmtB)

0.98

0.311

PII-like protein (GlnK)

1.11

0.301

glutamate dehydrogenase 
$\operatorname{gudB} \operatorname{gudB}$

Arginine metabolism

$\begin{array}{ll}\operatorname{rocA} & \operatorname{roc} A B C \\ \operatorname{roc} D & \operatorname{roc} D E F \\ \arg C & \operatorname{Arg} C J B D-c p a-F \\ \arg G & \arg G H \\ \operatorname{carA} & \operatorname{carAB}\end{array}$

\section{Urea cycle}

$\operatorname{aspB} \quad \operatorname{aspB}$

ans $A$ ans $A B$

\section{Other genes}

\begin{tabular}{|c|c|c|c|c|}
\hline pyrAA & pyr $A A A B$ & carbamoyl-phosphate synthetase & 0.94 & 0.060 \\
\hline pps & & phosphoenolpyruvate synthase & 1.08 & 0.188 \\
\hline crh & $y v c I J K L$ crh $y v c N$ & catabolite repression HPr-like protein & 0.94 & 0.091 \\
\hline
\end{tabular}

${ }^{a}$ The relative gene expression in the presence of succinate and glutamate is shown (CG medium: 1.0).

${ }^{b}$ The standard deviation includes values from three arrays that represent two biological experiments and one dye-flip experiment. pyrroline-5-carboxylate dehydrogenase

0.106

ornithine aminotransferase

N-acetylglutamate- $\gamma$-semialdehyde dehydrogenase

argininosuccinate synthase

carbamoyl-P-transferase

0.307

aspartate aminotransferase

1.10

0.121

L-asparaginase

1.09

carbamoyl-phosphate synthetase

0.060

0.091
267

106 


\section{Curriculum vitae}

\section{Personal Information}

Date of Birth

Place of Birth

Nationality

Parents

\section{School Education}

$1979-1983$

1983 - 1985

$1985-1993$

1993

\section{Activities before studies}

1993 - 1994

$1994-1996$

\section{Scientific Education}

$1996-2001$

$2001-2003$

$2002-2003$

Thesis title:

$10 / 2003-08 / 2006$

Thesis title:

since $08 / 2006$
May $2^{\text {nd }}, 1973$

Bamberg, Germany

German

Johann Schilling and Rosemarie Schilling

Primary School (Grundschule Walsdorf)

Secondary School (Hauptschule Walsdorf)

ETA-Hoffmann-Gymnasium Bamberg

General University Qualification (Abitur)

Civil Service, Caritas Verband Erlangen

Trainee in Financial Services, Sparkasse Bamberg

Studies of Biology and Chemistry at the University of Erlangen-Nuremberg Diploma Studies of Biology

Diploma thesis in the group of PD Dr. Jörg Stülke, at the University of Erlangen-Nuremberg

"Specific determinants of the interaction of the Antiterminator Protein GlcT with its target mRNA"

Ph.D. Student in the group of Prof. Dr. Jörg Stülke, at the University of Göttingen

"Carbon Catabolism in Bacillus subtilis: Global and Molecular Views on the Control of Gene Expression"

Employee at Miltenyi Biotec GmbH 